Aus der Klinik für Kinder- und Jugendmedizin

Prof. Dr. med. J. Gärtner

der Medizinischen Fakultät der Universität Göttingen

\title{
Quantitative Myelinbildgebung bei \\ Erkrankungen der weißen Hirnsubstanz im \\ Kindes- und Jugendalter
}

\author{
INAUGURAL-DISSERTATION \\ zur Erlangung des Doktorgrades \\ der Medizinischen Fakultät der \\ Georg-August-Universität zu Göttingen
}

vorgelegt von

Matthias Preuße

aus

Hamburg

Göttingen 2020 
Dekan:

\section{Betreuungsausschuss}

Betreuerin:

Ko-Betreuer:

\section{Prüfungskommission}

Referent/in:

Ko-Referent/in:

Drittreferent/in:
Prof. Dr. med. W. Brück

PD Dr. med. Steffi Dreha-Kulaczewski

PD Dr. rer. nat. Peter Dechent

PD Dr. rer. nat. Peter Dechent

Prof. Dr. med. Martin Oppermann

Datum der mündlichen Prüfung: 23.02.2021 
Hiermit erkläre ich, die Dissertation mit dem Titel "Quantitative Myelinbildgebung bei Erkrankungen der weißen Hirnsubstanz im Kindes- und Jugendalter" eigenständig angefertigt und keine anderen als die von mir angegebenen Quellen und Hilfsmittel verwendet zu haben.

Göttingen, den

(Unterschrift) 


\section{Inhaltsverzeichnis}

Abbildungsverzeichnis ............................................................................II

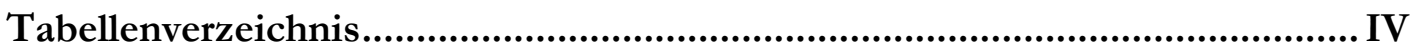

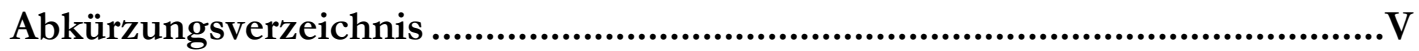

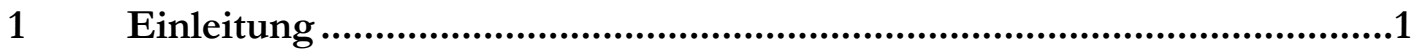

1.1 Kindliche weiße Hirnsubstanz in der Magnetresonanztomographie................................ 1

1.2 Erkrankungen der weißen Hirnsubstanz...................................................................... 5

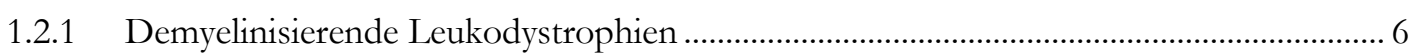

1.2.2 Hypomyelinisierende Leukodystrophien.................................................................... 7

1.3 Ausgangspunkt und Zielsetzung der Arbeit ..................................................................... 10

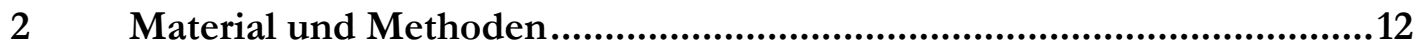

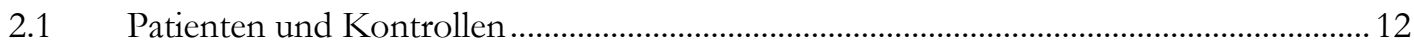

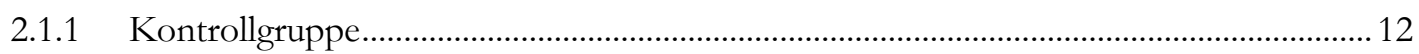

2.1.2 Kindliche-zerebrale-X-chromosomale-Adrenoleukodystrophie .....................................12

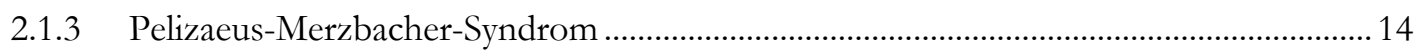

2.1.4 Zerebrale-Folatdefizienz ........................................................................................ 18

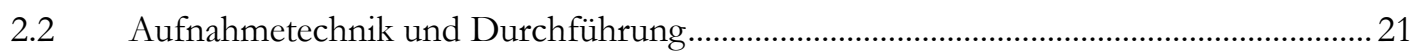

2.2.1 Aufnahmegerät und Sequenzen der Magnetresonanztomographie .................................2 21

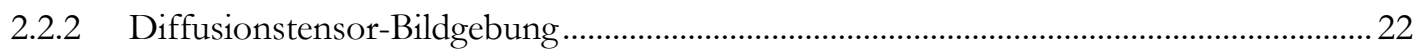

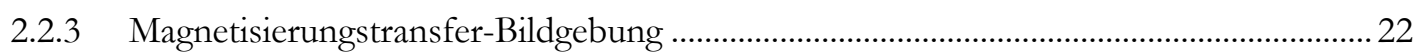

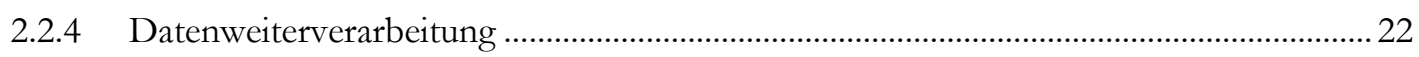

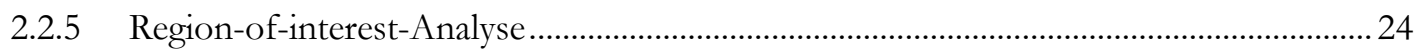

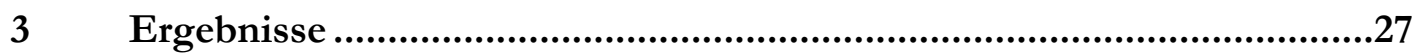

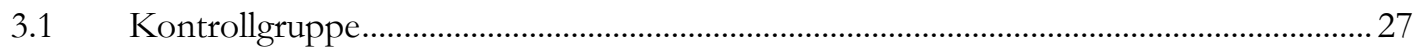

3.1.1 Verlaufsbeschreibung der Myelinparameter der Kontrollgruppe ……………...............27

3.2 Entzündliche Demyelinisierung - Kindliche-zerebrale-X-chromosomale-

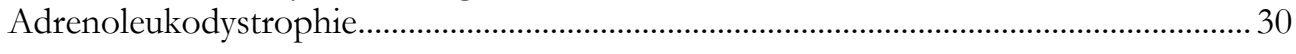

3.2.1 Verlaufsbeschreibung der Myelinparameter bei Patient 1 ................................................... 30

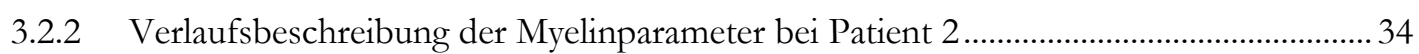

3.3 Hypomyelinisierung - Pelizaeus-Merzbacher-Syndrom..................................................... 37

3.3.1 Verlaufsbeschreibung der Myelinparameter der Patienten 3 bis 7 ................................... 37

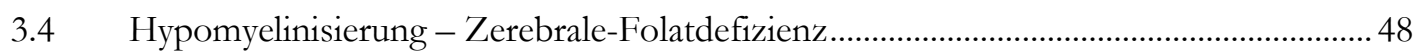

3.4.1 Verlaufsbeschreibung der Myelinparameter bei Patient 8 …………………................... 48

3.4.2 Verlaufsbeschreibung der Myelinparameter bei Patientin 9.............................................. 51

3.4.3 Verlaufsbeschreibung der Myelinparameter bei Patient 10.............................................. 54

3.4.4 Verlaufsbeschreibung der Myelinparameter bei Patientin 11 ............................................ 55

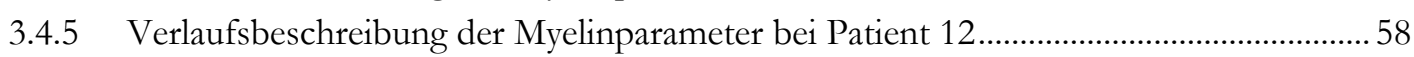




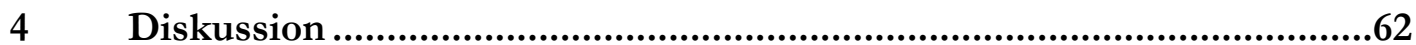

4.1 Bedeutung der quantitativen Magnetresonanztomographie ..........................................62

4.2 Prädiktionsfähigkeit der Kindlichen-zerebralen-X-chromosomalenAdrenoleukodystrophie. 63

4.3 Differenzierung von Krankheitsentitäten beim Pelizaeus-Merzbacher-Syndrom ..........66 66

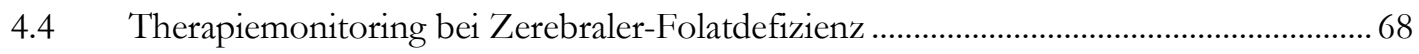

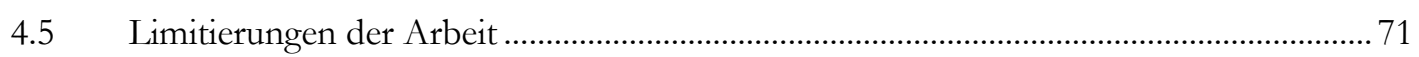

4.6 Relevanz der Ergebnisse und Ausblick ......................................................................

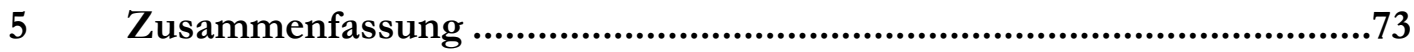

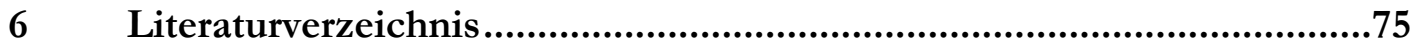

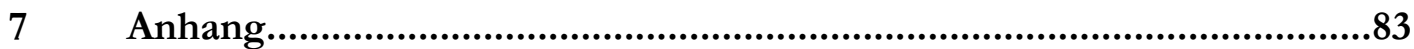




\section{Abbildungsverzeichnis}

Abb. 1: Die physiologische Myelinisierung im zeitlichen Verlauf...................................................... 4

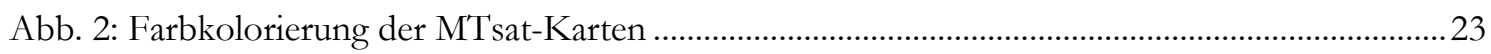

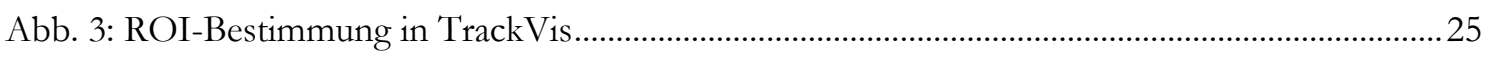

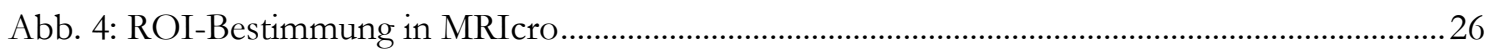

Abb. 5: Verlauf der Myelinisierung in der Kontrollgruppe ................................................................28

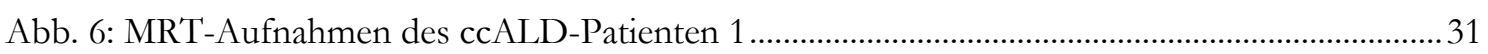

Abb. 7: Darstellung der Läsionen des Patienten 1 mit fünf Jahren .......................................................32

Abb. 8: Verlauf der myelinsensitiven Parameter der ccALD-Patienten 1-2 2.....................................34

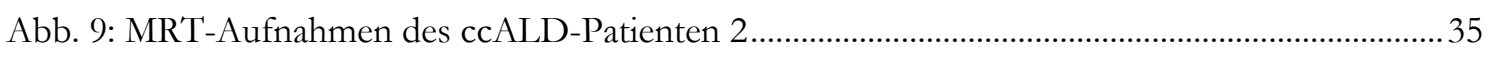

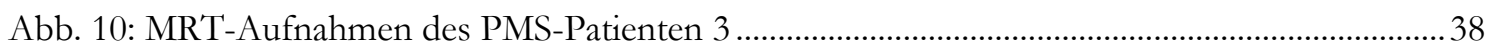

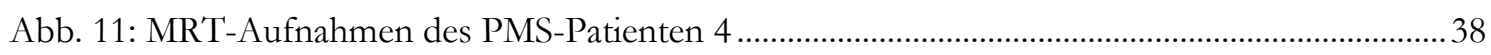

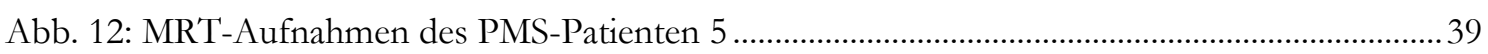

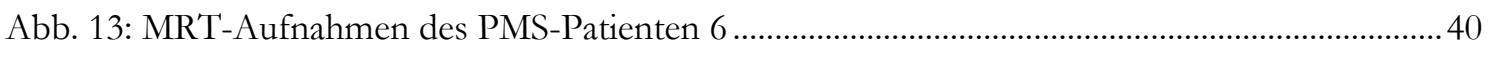

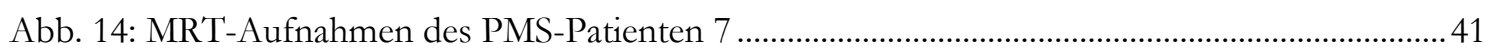

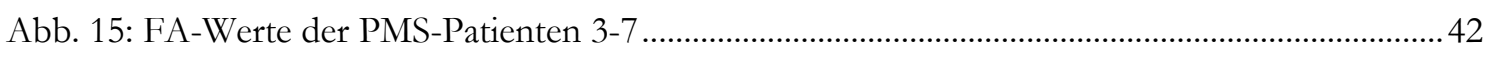

Abb. 16: DT- und MTsat-Werte der PMS-Patienten gestaffelt nach klinischer Symptomatik...........43

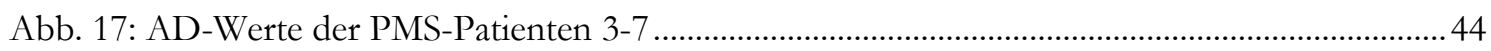

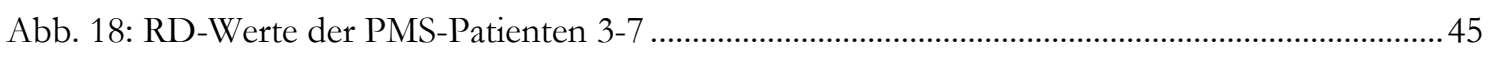

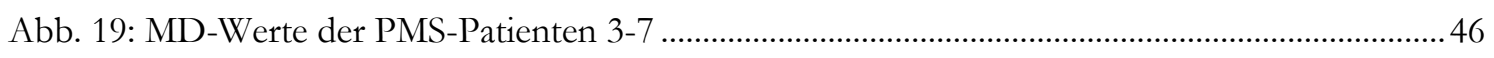

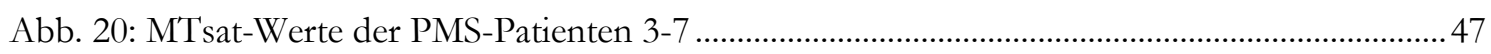

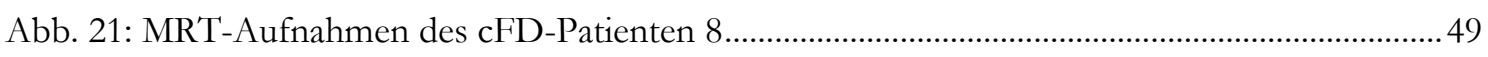

Abb. 22: ROI-Analyse der quantitativen DT- und MT-Parameter des cFD-Patienten 8 ...................51

Abb. 23: MRT-Aufnahmen der cFD Patientin 9 ...................................................................................52

Abb. 24: ROI-Analyse der quantitativen DT- und MT-Parameter der cFD-Patientin 9 ...................53

Abb. 25: MRT-Aufnahmen der cFD-Patientin 10..........................................................................5

Abb. 26: ROI-Analyse der quantitativen DT- und MT-Parameter der cFD-Patientin 10 ..................55

Abb. 27: MRT-Aufnahmen der cFD-Patientin 11............................................................................56

Abb. 28: ROI-Analyse der quantitativen DT- und MT-Parameter der cFD-Patientin 11 ..................57

Abb. 29: MRT-Aufnahmen des cFD-Patienten 12 .............................................................................5

Abb. 30: ROI-Analyse der quantitativen DT- und MT-Parameter des cFD-Patienten 12 .................61 


\section{Tabellenverzeichnis}

Tabelle 1: Übersicht über die Bildgebung der Kontrollgruppe ................................................................ 12

Tabelle 2: Übersicht der Klinik, Diagnostik und Therapie der ccALD-Patienten ..............................13

Tabelle 3: Übersicht Erstdiagnose und MRT-Untersuchungen der ccALD-Patienten .........................13

Tabelle 4: Übersicht der Klinik, Diagnostik und Therapie der PMS-Patienten ......................................14

Tabelle 5: Übersicht Erstdiagnose und MRT-Untersuchungen der PMS-Patienten.............................15

Tabelle 6: Klinische Einteilung der Patienten mit PMS....................................................................... 16

Tabelle 7: Modifizierter Funktionsbeeinträchtigungs-Score ..................................................................... 16

Tabelle 8: Punktzahl der Patienten mit PMS im Funktionsbeeinträchtigungs-Score............................17

Tabelle 9: Übersicht I der Klinik, Diagnostik und Therapie der Patienten mit cFD ............................18

Tabelle 10: Übersicht II der Klinik, Diagnostik und Therapie der Patienten mit cFD ........................19

Tabelle 11: Übersicht Erstdiagnose und MRT-Untersuchungen der Patienten mit cFD ....................2 21

Tabelle 12: Überblick konventionell-diagnostische und myelinsensitive MRT-Sequenzen ..................21

Tabelle 13: Lokalisationen der Region-of-interest nach Patientengruppen ...........................................24

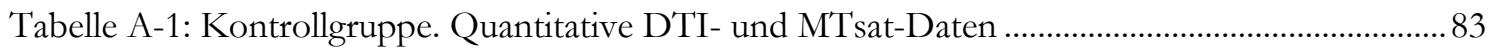

Tabelle A-2: ccALD-Patient 1. Quantitative DTI- und MTsat-Daten ...................................................8

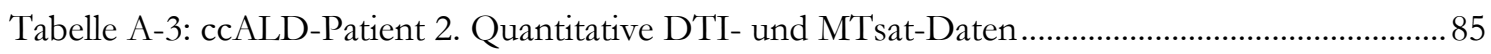

Tabelle A-4: PMS-Patienten 3-7. Quantitative DTI- und MTsat-Daten............................................... 85

Tabelle A-5: cFD-Patient 8. Quantitative DTI- und MTsat-Daten ...................................................... 87

Tabelle A-6: cFD-Patientin 9. Quantitative DTI- und MTsat-Daten.................................................... 89

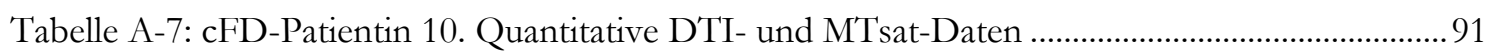

Tabelle A-8: cFD-Patientin 11. Quantitative DTI- und MTsat-Daten ............................................... 93

Tabelle A-9: cFD-Patient 12. Quantitative DTI- und MTsat-Daten.................................................... 94 


\section{Abkürzungsverzeichnis}

5-MTHF

a. u.

AD

ADC

AEP

AMN

ccALD

ccFA

cFD

cFTD

CSF

DT

DTI

DWI

EEG

EPI

FA

FDS

FLASH

FOLR

FR

FOLR1

FOLR2

FOLR3

FOLR4

GM

GvHR

HFM

HSZT
5-Methyltetrahydrofolat

Einheit für Absolutmessung, arbitrary units

axiale Diffusivität

scheinbarer Diffusionskoeffizient, apparent diffusion coefficient

akustisch evozierte Potenziale

Adrenomyeloneuropathie

Kindliche-zerebrale-X-Adrenoleukodystrophie, childhood cerebral X-linked

Adrenolenkodystrophie

farbkodierte (richtungskodierte) FA-Karte, color-coded FA-Karte

Zerebrale-Folat-Defizienz, cerebral folate deficiency

Zerebrale-Folattransport-Defizienz, cerebral folate transport deficiency

Zerebrospinalflüssigkeit, cerebrospinal fluid

Diffusionstensor, diffusion tensor

Diffusionstensor-Bildgebung, diffusion tensor imaging

Diffusions-gewichtete Bildgebung, diffusion weigbted imaging

Elektroenzephalografie

Echo-planar-imaging-Sequenz, echo planar imaging

fraktionale Anisotropie

Funktionsbeeinträchtigungs-Score, functional disability scoring

Fast-Low-Angle-Shot-Sequenz, fast low angle shot

Folatrezeptor-Gen

Folatrezeptor

Folatrezeptor alpha

Folatrezeptor beta

Folatrezeptor gamma

Folatrezeptor delta

Graue Hirnsubstanz, gray matter

Graft-versus-Host-Reaktion, graft-versus-host reaction

hereditäre Folat-Malabsorption, hereditary folate malabsorption

hämatopoetische Stammzelltransplantation 
KM

LBSL

LD

MD

MDS

MP-RAGE

MR

MRS

MRT

MT

MTsat

NAWM

NGS

NNR

p. u.

PCFT

PD

PLP1

PMS

PNS

$\mathrm{RD}$

RFC

ROI

SD

SNR

STEAM

T1

$\mathrm{T} 2$

VEP
Kontrastmittel

Leukoenzephalopathie-mit-Hirnstamm-und-Rückenmarksbeteiligung-und-

Laktaterhöhung, leukoencephalopathy with brainstem and spinal cord involvement and lacta elevation

Leukodystrophie(n)

mittlere Diffusivität

Motorischer-Entwicklungs-Score, motor developmental score

Magnetization-prepared-rapid-acquisition-of-gradient-echoes-Sequenz, magnetization prepared rapid acquisition of gradient echoes

Magnetresonanz

Magnetresonanzspektroskopie

Magnetresonanztomographie

Magnetisierungstransfer, magnetization transfer

Magnetisierungstransfer-Sättigung, magnetization transfer saturation

Normal-erscheinende-weiße-Hirnsubstanz, normal-appearing white matter

Next-Generation-Sequenzierung, next generation sequencing

Nebennierenrinde

Prozenteinheiten, percent unit

Protonen-gekoppelter-Folat-Transporter, proton-coupled folate transporter

Protonendichte

Proteolipidprotein-1

Pelizaeus-Merzbacher-Syndrom, Pelizaeus Merzbacher disease

Peripheres Nervensystem

radiale Diffusivität

Folattransporter-1, reduced folate carrier

Bereich von Interesse, region of interest

Standardabweichung, standard deviation

Signal-Rausch-Verhältnis, signal to noise ratio

Stimulated-echo-acquisition-mode-Sequenz, Stimulated echo acquisition mode

Zeitkomponente der Längsrelaxation, auch Längsrelaxationszeit

Zeitkomponente der Querrrelaxation, auch Querrrelaxationszeit

visuell evozierte Potenziale 
VLCFA Sehr-langkettige-Fettsäure, very long chain fatty acid

WM weiße Hirnsubstanz, white matter

WMLF Links frontale weiße Hirnsubstanz

WMLPO Links parieto-okzipitale weiße Hirnsubstanz

X-ALD X-chromosomale Adrenoleukodystrophie, X-linked Adrenoleukodystrophie

qMRT quantitative Magnetresonanztomographie

ZNS Zentrales Nervensystem

-w gewichtet 


\section{Einleitung}

\subsection{Kindliche weiße Hirnsubstanz in der Magnetresonanz- tomographie}

Das zentrale Nervensystem (ZNS) besteht aus grauer (GM, gray matter) und weißer Hirnsubstanz (WM, white matter). Der Großteil der Nervenfasern (Axone) verläuft durch die die WM. Die histopathologisch beschriebene weiße Farbe ist auf das fettreiche Myelin zurückzuführen (Dubois et al. 2014). Das Myelin bzw. die Myelinscheide wird von Oligodendrozyten gebildet, deren flache verlängerte Zellmembranen sich lamellenartig um die Axone winden und auf diese Weise eng gewundene Hüllen von Lipid- und Proteinlamellen formen. Neben den myelinisierten Axonen liegen in der WM eine Vielzahl von Astrozyten und Mikrogliazellen (Dubois et al. 2014).

Die bevorzugte Bildgebungsmodalität zur Beurteilung der WM ist die Magnetresonanztomographie (MRT) (van der Knaap und Valk 2005). In konventionellen MRTUntersuchungen werden u. a. Längsrelaxationszeit (T1)-gewichtete (,--w“), Querrelaxationszeit (T2)-w und Protonendichte (PD)-w Aufnahmen durchgeführt. Die Interaktion der Protonen mit ihrer Umgebung wird mit der T1 charakterisiert, die Wechselwirkungen zwischen Protonen mit der T2 (Dubois et al. 2014). Die T1 und T2 sowie die PD variieren mit den Gewebseigenschaften und stellen die Grundlage für die Erzeugung des Bildkontrastes der konventionellen MRT-Aufnahme dar (Trümmler 2011). Die ausgereifte fettreiche Myelinscheide hat eine sehr kurze T1 und stellt sich in T1-w Aufnahmen hyperintens (hell) dar. In T2-w Aufnahmen erscheint Gewebe mit hohem Fettanteil und kurzer T2, wie die WM bei fortgeschrittener Myelinisierung, hypointens (dunkel) (s. Abb. 1, D).

Die Kontraste in den T1-w und T2-w Aufnahmen von Neugeborenen und Säuglingen unterscheiden sich deutlich von denen von Kleinkindern und Erwachsenen (Dubois et al. 2014). Ursächlich dafür ist u. a. die noch nicht abgeschlossene Entwicklung der Myelinscheide und damit zusammenhängenden abweichenden Fett- und Wasseranteile im neonatalen Hirngewebe (Dubois et al. 2014).

Die Myelinisierung beginnt intrauterin im Gestationsalter von ungefähr 16 Wochen (Yakovlev und Lecours 1967). Zum Zeitpunkt der Geburt ist der Prozess im ZNS zumeist nur in posterioren Anteilen der Pons und in zentralen Bereichen des Cerebellum abgeschlossen (Baumann und Pham-Dinh 2001). In den ersten postnatalen Monaten erfolgt dann ein rasches Voranschreiten der Myelinisierung. Mit dem dritten postnatalen Monat sollte die Myelinisierung des hinteren Schenkels der Capsula interna und des Splenium corporis callosum (in der Folge Splenium) weitgehend abgeschlossen sein (s. Abb. 1, A) (Dubois et al. 2014; van der Knaap und Valk 2005). Obgleich sich einzelne myelinisierte Strukturen in T1-w Aufnahmen bereits hyperintens darstellten, erscheint die WM mit zum Großteil nicht- 
myelinisierten Fasern bis zum sechsten Lebensmonat in den T1-w Aufnahmen weitgehend hypointens und in den T2-w Aufnahmen hyperintens (s. Abb. 1, A) (Dubois et al. 2014; Yoshida et al. 2013). Am besten beurteilen lässt sich die WM von Säuglingen bis zum sechsten Lebensmonat im konventionellen MRT in T1-w Aufnahmen (Dubois et al. 2014; van der Knaap und Valk 2005). Mit weiter voranschreitender Myelinisierung verkürzen sich die T1 und die T2 der WM im MRT (Steenweg et al. 2010). Dabei tritt die T1-Verkürzung vor der T2-Verkürzung auf und ist stärker ausgeprägt (Barkovich 2000; Steenweg et al. 2010). Der Zeitraum vom sechsten bis zum zwölften Lebensmonat ist daher durch eine annähernde Intensität von WM und GM in den T2-w MRT-Aufnahmen gekennzeichnet (s. Abb. 1, B). Aufgrund der frühen Signaländerungen in T2-w Aufnahmen kann ein hohes T2-Signal in der WM auch gleichzeitig mit einer sich hypointens, isointens oder hyperintens darstellenden WM in den T1-w Aufnahmen auftreten (Steenweg et al. 2010). In der Beurteilung der WM sind vom sechsten bis zum zwölften Lebensmonat daher T2-w Aufnahmen vorzuziehen (Dubois et al. 2014; van der Knaap und Valk 2005). Abgeschlossen sein sollte bis zum Alter von einem Jahr spätestens die Myelinisierung des vorderen und hinteren Schenkels der Capsula interna, des Genu corporis callosum (in der Folge Genu), der Sehstrahlung (Radiatio optica) (s. Abb. 1, B) sowie der Hörstrahlung (Radiatio acustica) (Dubois et al. 2014). Die WM stellt sich ab dem ersten Lebensjahr T1-hyperintens und T2-hypointens dar (s. Abb. 1, C) (Dubois et al. 2014; Steenweg et al. 2010). Mit Ende des zweiten Lebensjahres sollte die makroskopisch sichtbare Myelinisierung der Axone beim Kind weitgehend abgeschlossen sein (s. Abb. 1, D) (Baumann und Pham-Dinh 2001; Yakovlev und Lecours 1967). Das Myelinisierungsmuster gesunder Kindern ist nun ähnlich zu dem von Erwachsenen (earlyadult pattern) (Dubois et al. 2014).

Eine zunehmende Bedeutung für die Beurteilung der Entwicklung gesunder, als auch für die Diagnostik und Therapiebeurteilung erkrankungsbedingt veränderter WM, gewinnt die quantitative Magnetresonanztomographie (qMRT). Aktuell werden in der Forschung Grundlagen für die Auswertung unterschiedlicher qMRT-Verfahren untersucht. Vorteil der qMRT gegenüber konventionellen MRT-Verfahren ist, dass sie nicht nur Informationen über das Volumen und die Makrostruktur der GM und WM sondern ebenfalls über die Mikrostruktur der WM liefern können (Cascio et al. 2007). Darüber hinaus besteht die Möglichkeit der Quantifizierung von Myelindefiziten. In einem Konsens-Artikel aus dem Jahr 2011 wurden drei qMRT-Verfahren zur Charakterisierung der mikrostrukturellen Eigenschaften der WM hervorgehoben (Alexander et al. 2011). Neben der DiffusionstensorBildgebung (DTI) wurden die Magnetisierungstransfer (MT)-Bildgebung und die Mehrkomponenten-Relaxometrie-Messung als vielversprechendste quantitative MRTMethoden zur Myelindarstellung aufgeführt. Im Rahmen dieser Arbeit wurde die kindliche WM mithilfe der DTI und MT-Bildgebung untersucht.

Die DTI ist eine Weiterentwicklung der Diffusions-gewichteten Bildgebung (DWI, diffusion weighted imaging) und baut darauf auf, dass Wassermoleküle im Gewebe unterschiedlich diffundieren und sich dieses quantitativ messen lässt. Die Ausbreitung hängt sowohl von den 
physikalischen Eigenschaften des Gewebes, als auch von der Behinderung der Molekülbewegung durch Zell- und Organellenmembranen ab (Dubois et al. 2014; Le Bihan und Johansen-Berg 2012). Bei der DTI wird im Gegensatz zur DWI nicht nur die Diffusivität der Wasserprotonen, sondern auch die Gerichtetheit der Diffusivität miteinbezogen (ErtlWagner und Wagner 2019). Der „scheinbare Diffusionskoeffizient“ (ADC, apparent diffusion coefficient) der DTI ist ein quantitativer Parameter, der bereits routinemäßig in der klinischen Praxis verwendet wird. Im Nachweis der akuten zerebralen Ischämie haben sich ADCKarten aufgrund der frühen Darstellbarkeit der Diffusionseinschränkung bei interstitiellem Ödem als wertvoll erwiesen (Wilke und Dreha-Kulaczewski 2019). Weitere Anwendungsfelder der DTI sind die Beurteilung von intrakraniellen Abszessen und Epidermoiden sowie die neurochirurgischen Operationsfeldplanung (Choudhri et al. 2014; Ertl-Wagner und Wagner 2019; Wilke und Dreha-Kulaczewski 2019).

Neben frei beweglichen Protonen können auch solche Protonen angeregt werden und ein Magnetresonanz (MR)-Signal abgeben, die nicht oder nur bedingt beweglich sind, weil sie an Proteine in der Myelin-Doppelschicht gebunden sind. Diese gebundenen Protonen werden als makromolekulare Protonen bezeichnet. Der MT charakterisiert die Menge und den Grad der Übertragung von Magnetisierung zwischen frei beweglichen (freier pool) und nichtbeweglichen Teilchen (gebundener pool) (Pouwels et al. 2014). Über die Messung des Austauschs der Magnetisierung von Protonen der Lipiddoppelschicht der Myelinmembran auf sich frei bewegenden Protonen im Interstitium ist eine indirekte Beschreibung des Myelingehalts möglich (Dreha-Kulaczewski et al. 2012). Der semiquantitative Parameter MT-Sättigung (MTsat) hat sich als besonders sensitiv bewiesen, um Veränderungen der Myelinisierung anzuzeigen (Helms et al. 2008). 


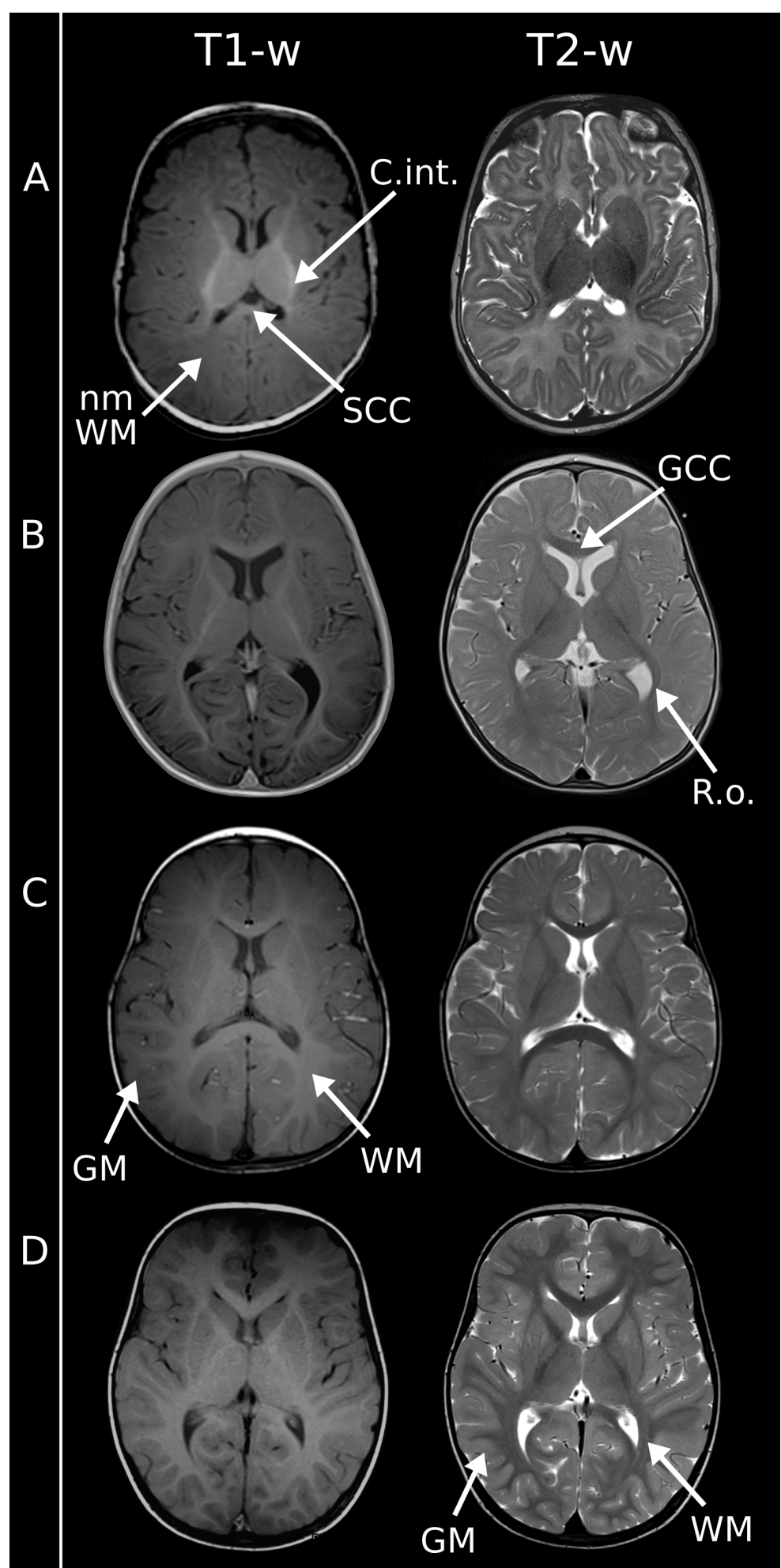

Abb. 1: Die physiologische Myelinisierung im zeitlichen Verlauf

A: Vier Monate alter männlicher Säugling. B: Zehn Monate alter weiblicher Säugling. C: 16 Monate altes Mädchen. D: 2,3 Jahre altes Mädchen. Weiße Pfeile: Nicht-myelinisierte weiße Hirnsubstanz (nm WM) und myelinisierte Capsula interna (C. int.), Genu (GCC), Sehstahlung (R. o.) und Splenium (SCC). Graue Hirnsubstanz (GM) und myelinisierte weiße Hirnsubstanz (WM).

Mit freundlicher Genehmigung von Prof. Dr. med. Ertl-Wagner und PD Dr. med. Born. 


\subsection{Erkrankungen der weißen Hirnsubstanz}

Durch den sprunghaften Erkenntnisgewinn von klinischer Präsentation und Pathophysiologie besonders durch die Methoden der Next-Generation-Sequenzierung (NGS, next generation sequencing) wurde die Definition von Leukodystrophien (LD) in den letzten Jahren wiederholt angepasst. Aktuell werden darunter monogenetische Erkrankungen zusammengefasst, die unabhängig vom Zelltyp, der Gewebskomponenten und der pathogenetischen Prozesse, primär die WM betreffen (van der Knaap et al. 2019).

LD können sich in jedem Lebensalter manifestieren (Schiller et al. 2019). Studien zur Prävalenz von kindlichen LD konnten eine jährliche Neuerkrankungsrate von bis zu 1:7.500 feststellen (Bonkowsky et al. 2010). LD weisen eine signifikante Heterogenität im Krankheitsverlauf und in extraneurologischen Manifestationen auf (Parikh et al. 2015). Erkrankte Kinder zeigen fast immer motorische Auffälligkeiten wie eine verspätete Aneignung von motorischen Meilensteinen, eine Stagnation der motorischen Entwicklung oder eine Regression der motorischen Fähigkeiten (Parikh et al. 2015). Bei Patienten, die erst im späteren Lebensalter Symptome entwickeln, sind erste Anzeichen häufig Gangunsicherheiten, Sturzneigungen oder Verschlechterungen der kognitiven Fähigkeiten (Parikh et al. 2015). Einige LD führen zu einem ausgeprägten Verlust des Kleinhirnvolumens und gehen mit einer fortschreitenden Ataxie einher. Darüber hinaus sind neurologische Manifestationen wie autonome Funktionsstörungen, Veränderungen des Kopfumfangs, Krampfanfälle oder Verhaltensstörungen wie extreme frühkindliche Reizbarkeit, Verschlechterung der schulischen Leistungen, neu einsetzende Hyperaktivität und Veränderungen der Persönlichkeit beschrieben (Parikh et al. 2015).

Die molekulargenetische Untersuchung und die kraniale MRT-Bildgebung sind neben der klinischen Untersuchung die wichtigsten Verfahren zur Diagnostik und Klassifizierung von LD (Schiller et al. 2019). Zu den häufigsten Ursachen angeborener LD gehören lysosomale Speichererkrankungen wie die metachromatische LD, peroxisomale Störungen wie die X-chromosomale Adrenoleukodystrophie (X-ALD), mitochondriale Dysfunktionen wie die Leukoenzephalopathie-mit-Hirnstamm-und-Rückenmarksbeteiligung-und-Laktaterhöhung (LBSL) sowie Defekte in Myelinproteinen wie beim Pelizaeus-Merzbacher-Syndrom (PMS) (Boespflug-Tanguy et al. 2008; Bonkowsky et al. 2010; van der Knaap und Valk 2005).

Der Begriff Dysmyelinisierung ist eine allgemeine Beschreibung einer abnormen oder fehlerhaften Myelinisierung (van der Knaap und Valk 2005). Davon abzugrenzen ist die Demyelinisierung dessen Charakteristikum der Verlust von zunächst normal ausgebildetem Myelin ist. Als hypomyelinisierende LD werden Erkrankungen mit von Geburt an reduzierter oder fehlender Myelinisierung zusammengefasst (Parikh et al. 2015). Eine hypomyelinisierende LD wird diagnostiziert, wenn bei einem Kind, das älter als ein Jahr alt ist, eine verminderte Myelinisierung festgestellt wird und in einer zweiten Untersuchung im Abstand von mindestens sechs Monaten keine Zunahme des Myelinvorkommens gezeigt werden kann (Schiffmann und van der Knaap 2009). 


\subsubsection{Demyelinisierende Leukodystrophien}

\subsubsection{X-chromosomale Adrenoleukodystrophie}

Die X-ALD (MIM \#300100) ist eine angeborene X-chromosomale Störung des Peroxysomen-Stoffwechsels, bei der sehr langkettigen Fettsäuren (VLCFA) akkumulieren und sich im Gewebe ablagern (Marchetti et al. 2018; Raymond et al. 2018). Die Prävalenz der Erkrankung liegt für hemizygote Männer zwischen 1:20.000 und 1:50.000 und für heterozygote Frauen zwischen 1:14.000 und 1:17.000 (Bezman et al. 2001; Marchetti et al. 2018). Sie manifestiert sich zumeist im Kleinkind- und Schulkindalter (Raymond et al. 2018).

Die der X-ALD zugrunde liegenden Mutationen sind im Gen ABCD1 (MIM \#300371) im langen Arm des X-Chromosoms (Xq28) lokalisiert. Das Gen kodiert für das gleichnamige Membranprotein, welches für den Transport der VLCFA in die Peroxysomen essentiell ist (Berger et al. 2014; Marchetti et al. 2018). Bis Februar 2020 waren über 840 unterschiedliche Mutationen in der „ALD-Mutationen Datenbank“, einem gemeinnützigen Online-Projektes des Amsterdam University Medical Centers, beschrieben. ${ }^{1}$

Können die VLCFA nicht in die Peroxysomen transportiert und mithilfe der B-Oxidation biochemisch abgebaut werden, kommt es zur Akkumulation der VLCFA. Dieses geschieht insbesondere in der Nebennierenrinde (NNR), in den Leydig-Zellen des Hodens und in den Myelinscheiden des PNS und ZNS (Marchetti et al. 2018; van der Knaap und Valk 2005). Diese Ansammlung von VLCFA scheint bei allen X-ALD-Typen von Bedeutung zu sein (Berger et al. 2014). Entzündungsprozesse gelten ebenfalls als entscheidend für den Krankheitsverlauf, der genaue Pathomechanismus ist bisher allerdings noch nicht verstanden (Berger et al. 2014; Marchetti et al. 2018). Bei der zerebralen Verlaufsform wird spekuliert, dass die Anhäufung der VLCFA die Apoptose der Oligodendrozyten über eine neuroinflammatorische Immunantwort mit vermehrter Expression von Zytokinen, interzellularen Adhäsionsmolekülen und oxidativem Stress induziert (Marchetti et al. 2018).

Es sind unterschiedliche Verlaufsformen der X-ALD beschrieben und die Erkrankung kann sich auch bei Betroffenen einer Familie phänotypisch sehr unterschiedlich manifestieren (Raymond et al. 2018). Zu den Verlaufsformen gehören die asymptomatische X-ALD, die Isolierte-Addison-Erkrankung, die Adrenomyeloneuropathie (AMN) und die Kindlichezerebrale-X-chromosomale-ALD (ccALD) (Engelen et al. 2012). Die AMN ist eine leichte Form der X-ALD und tritt in der Regel bei Männern zwischen dem 20. und 30. Lebensjahr auf (Gołębiowska et al. 2018). Die ccALD als schwerste Verlaufsform manifestiert sich zumeist zwischen dem vierten und achten Lebensjahr (Engelen et al. 2012; Raymond et al. 2018). Schätzungsweise entwickeln 35\% der Jungen mit X-ALD eine ccALD (Raymond et al. 2018). $\mathrm{Zu}$ initialen Manifestationen der ccALD zählen Verschlechterungen der

\footnotetext{
${ }^{1}$ https://adrenoleukodystrophy.info/mutations-and-variants-in-abcd1 (zuletzt abgerufen: 02.02.2020)
} 
schulischen Leistungen, Verhaltensauffälligkeiten, Worttaubheit, abnehmende Sehschärfe, Paresen der Gliedmaßen, Apraxien sowie zerebellare Ataxien und Krampfanfälle. Nach dem ersten Auftreten klinischer Symptome zeigt sich meist ein rasches Voranschreiten der Erkrankung mit zunehmenden neurologischen Ausfällen (Engelen et al. 2012). Bei Erkrankten bilden sich demyelinisierende Läsionen im ZNS. Der Manifestationsort ist in 80\% der Fälle das Splenium (Eichler et al. 2007; van der Knaap und Valk 2005). Eine weitere häufige primäre Lokalisation ist die Pyramidenbahn. Bei weiteren zehn bis 15 Prozent der Patienten mit ccALD zeigt die Demyelinisierung eine anteriore Dominanz mit häufig erstem Auftreten im Genu (Nowak et al. 2015). Mädchen und Frauen mit X-ALD bleiben häufig asymptomatisch oder zeigen einen sehr milden Verlauf (Engelen et al. 2012; O'Neill et al. 1984).

Die Therapie der X-ALD richtet sich nach der Symptomatik und der Verlaufsform. Bei Bestehen einer NNR-Insuffizienz aufgrund von Ablagerungen der VLCFA ist eine Therapie mit Kortikosteroiden unerlässlich (Raymond et al. 2018). Die Standardtherapie der ccALD ist derzeit die hämatopoetische Stammzelltransplantation (HSZT) (Eichler et al. 2017). Bei frühem Nachweis von Läsionen kann mittels HSZT mit einer Latenz von einigen Monaten ein Voranschreiten der Demyelinisierung verhindert werden (Eichler et al. 2017). Wichtige prognostische Faktoren für den Therapieerfolg sind das frühzeitige Erkennen der Läsionen und die schnelle Therapieeinleitung (Eichler et al. 2017). Ein großes Problem der HSZT ist die verhältnismäßig hohe posttherapeutsche Mortalitätsrate (Vogel et al. 2015). Dieses liegt insbesondere an Graft-versus-Host-Reaktionen (GvHR) sowie Infektionserkrankungen infolge der Immunsuppression vor und nach der Transplantation (Cartier und Aubourg 2010; Eichler et al. 2017). Neuere vielversprechende Behandlungsansätze sind autologe Exvivo-Gentherapien. Dabei werden Patienten hämatopoetischen CD34+ Stammzellen transplantiert, denen zuvor ein Vektor mit lentiviraler $A B C D 1$-Komplementär-DNA transduziert wurde (Cartier und Aubourg 2010; Eichler et al. 2017).

\subsubsection{Hypomyelinisierende Leukodystrophien}

\subsubsection{Pelizaeus-Merzbacher-Syndrom}

Das PMS (MIM \#312080) ist eine klassische hypomyelinisierende Erkrankung mit fehlerhaft gebildetem Proteolipidprotein 1 (PLP1) (Hudson et al. 1989). Der überwiegende Anteil der Erkrankten ist aufgrund der X-chromosomalen Vererbung männlich, jedoch sind auch symptomatische heterozygote Trägerinnen beschrieben (Osório und Goldman 2018). Bei männlichen Neugeborenen liegt die Prävalenz des PMS zwischen 1:200.000 bis 1:500.000 und die Inzidenz bei 1-2:100.000 (Bonkowsky et al. 2010; Numata et al. 2014).

In mehr als der Hälfte der Fälle ist die genetische Ursache des PMS eine Duplikation des auf dem X-Chromosom (Xq21.3-Xq22) liegenden PLP1-Gen (MIM \#300401). Es kann jedoch auch eine Deletion, eine Missense-, Frameshift-, Insertions- oder Nonsense-Mutation des genannten Gen ursächlich sein (Barkovich 2005; Hobson und Garbern 2012; Osório et al. 
2017; Woodward et al. 1999). Das PLP1-Gen kodiert neben dem PLP1 auch für dessen alternativ gespleißte Isoform, das Protein DM20 (Hobson und Kamholz 2018).

Das PLP1 ist eines der wichtigsten Transmembranproteine der Myelinscheide und sowohl an der Differenzierung und Kompaktierung der Oligodendrozyten, als auch an der Stabilisierung und Aufrechterhaltung der Myelinmembran beteiligt (Hobson und Kamholz 2018). Bei Veränderungen im PLP1-Gen kommt es abhängig von der Mutation zur PLP1/DM20-Überexpression und Sequestrierung in den Lysosomen oder zur Akkumulation von falsch gefalteten Proteinen im endoplasmatischen Retikulum (Osório und Goldman 2018). Der genaue Pathomechanismus ist noch nicht verstanden. Fest steht jedoch, dass die genetische Veränderung zu dysfunktionalen Oligodendrozyten und darüber zu einem Fehlaufbau der Myelinscheide und Myelindefizit führt (Hobson und Kamholz 2018; Hoffman-Zacharska et al. 2013; Osório und Goldman 2018). Da das PLP1 am axonalen Überleben beteiligt ist, ist ebenfalls eine axonalen Degeneration beim PMS beschrieben (Garbern et al. 1997; Osório und Goldman 2018).

Das Spektrum der PLP1-assozierten Erkrankungen weist unterschiedliche phänotypische Erscheinungen auf (Seitelberger 1995). Zum PMS werden das klassische PMS (ORPHA:280219, Typ I), das schwere konnatale PMS (ORPHA:280210, Typ II) sowie die in der klinischen Symptomatik dazwischen liegende Übergangsform (transitionale Form) (ORPHA:280224, Typ III) gezählt. Das klassische PMS ist mit 70\% die häufigste Verlaufsform. Die Übergangsform und das schwere konnatale PMS machen jeweils 15\% der PMS-Erkrankungen aus (Hobson und Kamholz 2018). Die ersten Symptome des klassischen PMS treten typischerweise innerhalb der ersten fünf Lebensjahre auf. Nystagmen, muskuläre Hypotonien, Tremor und epileptische Anfälle gehören zu den initialen Symptomen, zu denen im Verlauf Dysphagien, Spastiken, dystone Körperhaltungen und Ataxien hinzukommen können. Die Sprache der Patienten ist häufig verlangsamt und die kognitiven Fähigkeiten sind beeinträchtigt (Hobson und Kamholz 2018). Bei der konnatalen Form liegt typischerweise bereits bei Geburt ein Nystagmus und eine ausgeprägte muskuläre Hypotonie vor. Innerhalb des ersten Lebensjahres kommen häufig Spastiken, extrapyramidale Hyperkinesien, Schluckschwierigkeiten und epileptische Anfälle hinzu (van der Knaap und Valk 2005). Teilweise werden bei Patienten mit PMS innerhalb der ersten zehn Lebensjahre motorische Entwicklungsfortschritte beobachtet, im Verlauf kommt es jedoch stets zur Stagnation und Regression (Sarret et al. 2016). Das zweite Lebensjahrzehnt der Patienten ist, sofern dieses Alter erreicht wird, gekennzeichnet durch neurologische Verschlechterungen bis hin zur schweren Tetraplegie (Sarret et al. 2018). Hinweise auf ein PMS geben im MRT die globale Hypomyelinisierung sowie Atrophien im Cerebellum und im Corpus Callosum (Sumida et al. 2016). Patientinnen mit PMS zeigen im Gegensatz zu den männlichen Betroffenen einen milden Verlauf (Hobson und Kamholz 2018; Hurst et al. 2006).

Die Therapie des PMS ist aktuell auf symptomatische und supportive Maßnahmen begrenzt. Erste medikamentöse und genmodulierende off-label Therapien sind jedoch in der Testung 
(Marteyn et al. 2016; Osório et al. 2017; Osório und Goldman 2018). Vielversprechend zeigte sich der Ansatz humane, fetale neuronale Stammzellen Patienten mit PMS intrazerebral zu transplantierten und dabei mutierte Oligodendrozyten durch nicht-mutierte zu ersetzen (Goldman et al. 2012; Uchida et al. 2012; Wang et al. 2013; Windrem et al. 2008). Eine weitere Therapiemöglichkeit scheint die Transplantation von hämatopoetischen Stammzellen aus der Nabelschnur zu sein (Wishnew et al. 2014). Aktuell läuft dazu eine klinische Studie (NCT02254863). Ebenfalls in der Testung sind pharmakologische Ansätze mit Ionaprisan, Cholesterin und Curcumin (Osório und Goldman 2018).

\subsubsection{Zerebrale-Folat-Defizienz}

Die Zerebrale-Folat-Defizienz (cFD) ist ein klinisch heterogenes, neurometabolisches Krankheitsbild, das durch eine verminderte Konzentration von 5-Methyltetrahydrofolsäure (5-MTHF) in der Zerebrospinalflüssigkeit (CSF) gekennzeichnet ist (Ramaekers et al. 2012). Das 5-MTHF ist der aktive Metabolit der Folsäure (Folat). Die Prävalenz der cFD liegt zwischen 1:4.000 und 1:6.000 (Ramaekers et al. 2012). Als Ursache der angeborenen cFD sind sowohl Mutationen in für zerebrale Folatrezeptoren kodierenden Genen, als auch Autoantikörper, die sich gegen die zerebrale Folatrezeptoren richten, mitochondriale Defekte und Störungen im fetalen Folatmetabolismus beschrieben (Al-Baradie und Chudary 2014; Grapp et al. 2012; Pérez-Dueñas et al. 2011; Ramaekers et al. 2012; Steinfeld et al. 2009). Genetisch bedingte Erkrankungen mit Störung des zerebralen Folattransportes sind u. a. die zerebrale Folattransport-Defizienz (cFTD, OMIM \#613068, ORPHA \#217382) und die hereditäre Folat-Malabsorptionstörung (HFM, OMIM \#229050, OPRHA \# 90045). Im Jahr 2009 wurde mit der FOLR1-Mutation (MIM \#136430) die erste genetische Ursache für einen hirnspezifischen zerebralen Folattransport-Defekt entdeckt und die Erkrankung als cFTD bezeichnet (Steinfeld et al. 2009). Die der cFTD zugrunde liegende FOLR1Mutation führt zu einem Defekt des für den Transport wichtigen Folatrezeptor alpha (FOLR1). Daneben sind weitere Mutation in für zerebrale Folattransporter kodierenden Genen beschrieben, die jedoch nicht hirnspezifisch sind. Betroffenen sein können u. a. das SLC46A1-Gen, welches für den Protonen-gesteuerten Folat-Transporters (PCFT) kodiert (Zhao et al. 2009), das SLC19A1-Gen, das für den Folattransporter-1 (RFC) kodiert (Qiu et al. 2006) sowie das für die Methenyltetrahydrofolate-Synthetase kodierende MTHFD1-Gen (Watkins et al. 2011). Der Funktionsverlust des PCFT führt zur HFM (Aluri et al. 2018). Da der PCFT sowohl an den Enterozyten im Duodenum und proximalen Jejunum als auch an den Endothelzellen der Blut-Liquor-Schranke lokalisiert ist, führt ein Defekt sowohl zu einer verminderten peripheren als auch einer zerebralen Folat-Konzentration.

Der menschliche Körper besitzt nicht die Fähigkeit Folsäure selbstständig zu synthetisieren. Er ist auf die Aufnahme aus der Nahrung angewiesen (Balashova et al. 2018). Folsäure wird in Form von Dihydrofolat, Tetrahydrofolat oder 5-MTHF im Duodenum und Jejunum hauptsächlich mithilfe des PCFT und des RCF aufgenommen und über die Blutbahn verteilt (Balashova et al. 2018; Matherly und Hou 2008). Für den Folsäuretransport über die 
Zellmembran sowie für die Überquerung der Blut-Hirn- und Blut-Plazenta-Schranke sind neben dem PCFT, der RFC, der FOLR1, der Folatrezeptor beta (FOLR2), gamma (FOLR3) und delta (FOLR3) essentiell (Balashova et al. 2018; Pope et al. 2019). Intrazellulär wird Folsäure und deren Derivate mithilfe der Dihydrofolatreduktase zu 5-MTHF, 5,10Methylentetrahydrofolat oder 10-Formyltetrahydrofolat umgewandelt (Desmoulin et al. 2012). Fehlt im ZNS das 5-MTHF als Methylgruppendonator zur Remethylierung des SAdenosyl-Homozystein zu S-Adenosylmethionin, kann Cholin die erforderliche Methylgruppe zur Regeneration liefern. Das Cholin fehlt dann jedoch wiederum in der Synthese von Phosphatidylcholin und Sphingomyelin, welches wichtige Komponenten der Myelinmembran sind. Während der Gehirnentwicklung ist der Bedarf an Cholin besonders hoch und ein Mangel bedingt durch die notwendige Kompensation des 5-MTHF-Defizites führt zu einer gestörten Myelinisierung (Steinfeld et al. 2009).

Erste klinische Anzeichen der cFD und cFTD zeigen sich zwischen dem zweiten Lebensmonat und dem fünften Lebensjahr (Median: zweites Lebensjahr) (Pope et al. 2019). Initiale Symptome sind Muskelzittern, Ataxien, muskuläre Hypotonien sowie motorische und sprachliche Entwicklungsverzögerungen (Pope et al. 2019). Im Verlauf zeigen sich häufig Seh- und Hörstörungen, Dyskinesien, kognitive Retardierungen, spastische Paraplegien und eine Neigung zu epileptischen, meist myoklonischen Krampfanfällen (AlBaradie und Chudary 2014; Grapp et al. 2012; Pérez-Dueñas et al. 2011; Pope et al. 2019). Patienten mit HFM präsentieren zumeist innerhalb der ersten Lebensmonate eine megaloblastäre Anämie, eine Panzytopenie, rezidivierende Infekte, Diarrhoen sowie im Verlauf motorische, sensorische und kognitive Defizite. Viele Patienten leiden an epileptischen Krampfanfällen (Erlacher et al. 2015; Pope et al. 2019).

Der therapeutische Ansatz der cFTD ist die frühe Supplementation von 5-Formyltetrahydrofolat (Folinsäure) (Akiyama et al. 2015; Steinfeld et al. 2009). Innerhalb von sechs bis zwölf Monaten ist unter konsequenter Therapie eine Normalisierung des 5-MTHF-Spiegel im CSF und eine deutliche Besserung der klinischen Symptomatik zu erwarten (Grapp et al. 2012; Steinfeld et al. 2009). Sofern die Therapie frühzeitig begonnen wird, ist ein asymptomatischer Verlauf ohne neurologische Symptomatik möglich (Grapp et al. 2012; Ramaekers et al. 2013). Die HFM wird ebenfalls mit Folinat-Substitution therapiert (Kronn und Goldman 2018). Das B-Vitamin Folsäure selbst ist nicht liquorgängig und hat bei cFD daher keine therapeutische Relevanz.

\subsection{Ausgangspunkt und Zielsetzung der Arbeit}

Im klinischen Alltag verläuft die Beurteilung und Abschätzung des Myelindefizites aktuell zumeist qualitativ und beruht auf dem Vergleich der Signalstärke der WM und GM in konventionellen MRT-Aufnahmen. Der genauen Quantifizierung wird eine große Bedeutung u. a. zur Diagnostik, zur Festlegung des Zeitpunktes des Therapiebeginns und zum Therapie-Monitoring beigemessen. 
In dieser Arbeit wurden mithilfe der quantitativen und semiquantitativen myelinsensitiven diffusions-gewichteten DTI sowie der MT-Bildgebung der Status der Myelinisierung von Patienten mit drei kindlichen LD (ccALD, PMS und cFD) retrospektiv evaluiert. Ziel war es, Zusatzinformationen der quantitativen Myelinbildgebung $\mathrm{zu}$ den konventionelldiagnostischen MR-Sequenzen herauszuarbeiten. Dazu wurden die fraktionale Anisotropie (FA), die axiale Diffusivität (AD), die radiale Diffusivität $(\mathrm{RD})$ und die mittlere Diffusivität (MD) sowie der MT-Parameter MTsat bei zwölf Patienten im Corpus Callosum, in der Pyramidenbahn sowie in der frontalen und okzipitalen WM gemessen. Es wurde den Fragen nachgegangen, ob erstens mithilfe der DTI und MT-Bildgebung eine frühere Diagnosestellung der ccALD als mit der konventionellen Bildgebung möglich ist, inwiefern der natürliche Verlauf der Myelinisierung beim PMS gezeigt werden und ob der Effekt der myelinmodulierenden Therapie bei cFD gemessen werden kann. 


\section{$2 \quad$ Material und Methoden}

\subsection{Patienten und Kontrollen}

Insgesamt wurden zwölf Patienten mit symptomatischer LD sowie 36 gesunde oder asymptomatische Probanden (Kontrollgruppe) untersucht. Die Studie wurde durch die Ethikkommission der Universitätsmedizin Göttingen genehmigt. Für alle Patienten lag zum Zeitpunkt der Untersuchung eine Einwilligung der Patienten bzw. der Erziehungsberechtigten zur Teilnahme an der Studie vor. Im Folgenden sind die Charakteristika der einzelnen Gruppen näher beschrieben.

\subsubsection{Kontrollgruppe}

Die Kontrollgruppe umfasste elf weibliche und 25 männliche Probanden, die zwischen einem Jahr und 15 Jahren alt waren (s. Tabelle 1). Diffusionstensor (DT)-Daten lagen von 22 Probanden (sieben weiblich, 15 männlich) vor. $\mathrm{Zu}$ den 36 Probanden zählten fünf Probanden, bei denen eine asymptomatische X-ALD vorlag. Bei asymptomatischen Patienten ohne klinische, laborchemische und bildmorphologische Auffälligkeiten ist die Einordnung in die Kontrollgruppe zulässig. Die DTI wurde mit zwei unterschiedlichen Aquisitionstechniken durchgeführt, zum einen mit einer Stimulated-echo-acquisition-mode (STEAM)-Sequenz zum anderen mit einer Echo-Planar-Imaging (EPI)-Sequenz (s. Kapitel 2.2.2). Mit der STEAM-Sequenz wurden die DT-Daten von 19 Patienten aufgenommen (sechs weiblich, 13 männlich). Bei drei Messungen (eine weiblich, zwei männlich) wurde eine EPI-Sequenz verwendet. MT-Daten lagen von 31 Patienten (neun weiblich, 22 männlich) vor (s. Tabelle 1).

Tabelle 1: Übersicht über die Bildgebung der Kontrollgruppe

\begin{tabular}{llll}
\hline & Anzahl & Alter & Geschlecht \\
\hline Kontrollen insgesamt & 36 & $1,6-14,5$ Jahre & 11 weiblich, 25 männlich \\
DTI & 22 & $1,6-14,5$ Jahre & 7 weiblich, 15 männlich \\
$\quad$ STEAM & 19 & $2,5-14,4$ Jahre & 6 weiblich, 13 männlich \\
$\quad$ EPI & 3 & $1,6-14,5$ Jahre & 1 weiblich, 2 männlich \\
MT Bildgebung & 31 & $1,6-14,4$ Jahre & 9 weiblich, 22 männlich \\
\hline
\end{tabular}

\subsubsection{Kindliche-zerebrale-X-chromosomale-Adrenoleukodystrophie}

Die Patientengruppe ccALD umfasste zwei Patienten. Nachdem Geschwister beider Patienten die Erkrankung X-ALD diagnostiziert bekamen, wurden die Patienten dieser Studie ebenfalls molekulargenetisch getestet. So war die frühzeitige Diagnosestellung möglich. Bei Patient 1 lag die Mutation c.1661G >A im ABCD1-Gen vor, bei Patient 2 wurde ebenfalls eine Mutation im $A B C D 1$-Gen festgestellt. In Folge der Diagnose wurden in der frühen Kindheit beginnend regelmäßige endokrinologische, neurophysiologische und 
bildgebende Untersuchungen bei den zwei Patienten durchgeführt. MRT-Untersuchungen erfolgten im sechsmonatigen Intervall (s. Tabelle 3). Bei Patient 1 fiel drei Jahre und bei Patient 2 eineinhalb Jahre nach der molekulargenetischen Diagnose X-ALD eine T2hyperintense Läsion im Splenium mit (Patient 1) bzw. ohne Kontrastmittel (KM)-Aufnahme (Patient 2) im MRT auf. Damit bestätigte sich der Beginn der zerebralen Verlaufsform ccALD. Beide Patienten wurden zeitnah einer HSZT unterzogen.

Tabelle 2: Übersicht der Klinik, Diagnostik und Therapie der ccALD-Patienten

\begin{tabular}{|c|c|c|}
\hline & Patient 1 (Verlauf 2,5 bis 10,8 J.) & Patient 2 (Verlauf 5,2 bis $10,8 \mathrm{~J}$.) \\
\hline Genetische Veränderung & c.1661G >A (p.R554H) im $A B C D 1-G e n$ & Mutation im $A B C D 1-G e n *$ \\
\hline $\begin{array}{l}\text { Neurologische } \\
\text { Symptome }\end{array}$ & keine & keine \\
\hline Familienanamnese & positiv (Bruder ccALD) & positiv (Bruder ccALD) \\
\hline Endokrinologie & $\begin{array}{l}\text { NNR-Insuffizienz (ED 5,7 J.) } \\
\text { Therapie mit Hydrokortison }\end{array}$ & $\begin{array}{l}\text { NNR-Insuffizienz (ED 5,2 J.) } \\
\text { Therapie mit Hydrocortison und } \\
\text { Fludrokortison }\end{array}$ \\
\hline Stoffwechseldiagnostik & Erhöhung der VLCFA (ED 5,7 J.) & Erhöhung der VLCFA (ED 5,2 J.) \\
\hline $\begin{array}{l}\text { Neurophysiologische } \\
\text { Untersuchung } \\
\text { AEP, VEP }\end{array}$ & $\begin{array}{l}\text { EEG und VEP Normalbefund, links } \\
\text { zwischenzeitlich retrokochleäre } \\
\text { Leitungsstörung }\end{array}$ & $\begin{array}{l}\text { EEG und VEP Normalbefund, links } \\
\text { verlängerte retrokochleäre } \\
\text { Leitungsstörung und im Hirnstamm } \\
\text { rechts }\end{array}$ \\
\hline Erstes MRT & $\begin{array}{l}\text { atypisch lokalisierte, subkortiko-okzipitale } \\
\text { Signalauffälligkeit, nach MRS-Kontrolle } \\
\text { nicht als beginnende zerebrale Läsion } \\
\text { gewertet }\end{array}$ & keine zerebralen Auffälligkeiten \\
\hline $\begin{array}{l}\text { MRT bei Diagnose der } \\
\text { ccALD }\end{array}$ & $\begin{array}{l}\text { T1-hypointense und T2-hyperintense } \\
\text { Läsion im Splenium mit KM-Aufnahme } \\
\text { (Größe circa } 5 \mathrm{~mm} \text { ) - Alter 5,6 J. }\end{array}$ & $\begin{array}{l}\text { T1-hypointense und T2-hyperintense } \\
\text { Läsion im Splenium ohne KM-Aufnahme } \\
\text { (Größe circa } 3 \mathrm{~mm} \text { ) - Alter 6,8 J. }\end{array}$ \\
\hline Therapie (Alter) & $\begin{array}{l}\text { HSZT }(5,7 \mathrm{~J} .) \text { - post-OP: keine } \\
\text { Komplikationen }\end{array}$ & $\begin{array}{l}\text { HSZT }(7,0 \mathrm{~J} .) \text { - post-OP: rezidivierende } \\
\text { Paukenergüße, Otitiden, Konjunktividen } \\
\text { (Wertung als milde GvHR) }\end{array}$ \\
\hline
\end{tabular}

AEP: Akustisch evozierte Potenziale, ED: Erstdiagnose, EEG: Elektroenzephalographie, J.: Jahre, MRS: Magnetresonanzspektroskopie, NNR: Nebennierenrinde, VEP: Visuell evozierte Potentiale. *nicht genauer bekannt

Tabelle 3: Übersicht Erstdiagnose und MRT-Untersuchungen der ccALD-Patienten

\begin{tabular}{lllll}
\hline Patient & ED X-ALD & ED ccALD & Alter erstes, letztes MRT & Anzahl MRT-Untersuchungen \\
\hline Patient 1 & 2,5 J. & $5,6 \mathrm{~J}$. & 2,5 J., 7,3 J. & 9 \\
Patient 2 & $5,2 \mathrm{~J}$. & $6,8 \mathrm{~J}$. & $5,2 \mathrm{~J} ., 10,8 \mathrm{~J}$. & 7 \\
\hline
\end{tabular}

ED: Erstdiagnose, J.: Jahre 


\subsubsection{Pelizaeus-Merzbacher-Syndrom}

Die Patientengruppe PMS bestand aus fünf Patienten zwischen eineinhalb und elf Jahren (s. Tabelle 4 und 5). Die Diagnose erfolgte bei allen Patienten nach klinisch diagnostizierter psychomotorischer Entwicklungsverzögerung, dem Nachweis der Hypomyelinisierung im kranialen MRT und einer Mutation im PLP1-Gen.

Tabelle 4: Übersicht der Klinik, Diagnostik und Therapie der PMS-Patienten

\begin{tabular}{|c|c|c|c|c|c|}
\hline & $\begin{array}{l}\text { Patient } 3 \\
\text { (7,9 J.) }\end{array}$ & $\begin{array}{l}\text { Patient } 4 \\
(1,5 \mathrm{~J} . \text { bis } 6,9 \mathrm{~J} .)\end{array}$ & $\begin{array}{l}\text { Patient } 5 \\
(3,2 \mathrm{~J} . \text { bis } 4,1 \mathrm{~J} .)\end{array}$ & $\begin{array}{l}\text { Patient } 6 \\
(2,4 \text { bis } 4.7 \mathrm{~J} .)\end{array}$ & $\begin{array}{l}\text { Patient } 7 \\
(11,2 \mathrm{~J} .)\end{array}$ \\
\hline $\begin{array}{l}\text { Genetische } \\
\text { Veränderung }\end{array}$ & $\begin{array}{l}\text { c. } 817 \mathrm{C}>\mathrm{T} \\
\left(\mathrm{p} . \mathrm{R} 273^{*}\right) \\
\text { (Nonsense) }\end{array}$ & $\begin{array}{l}\text { p.Y207H } \\
\text { (Missense) }\end{array}$ & $\begin{array}{l}\text { c. } 650 G>A \\
\text { (p.G217D) } \\
\text { (Missense) }\end{array}$ & Duplikation* & Duplikation* \\
\hline $\begin{array}{l}\text { Beginn } \\
\text { Symptome }\end{array}$ & circa $1,5 \mathrm{~J}$ & Geburt & Geburt & Geburt & Geburt \\
\hline Nystagmus & nein & $\begin{array}{l}\text { kongenital, } \\
\text { intermittierend }\end{array}$ & kongenital & kongenital & nein \\
\hline$\frac{\text { Verlauf }}{\text { Kognition }}$ & $\begin{array}{l}\text { Probleme } \\
\text { Merkfähigkeit, } \\
\text { Konzentration, } \\
\text { Lernschwäche }\end{array}$ & $\begin{array}{l}\text { eingeschränkte } \\
\text { kognitive } \\
\text { Entwicklung. } \\
\text { Ausgeprägte } \\
\text { Leseschwäche }\end{array}$ & $\begin{array}{l}\text { eingeschränkte } \\
\text { kognitive } \\
\text { Entwicklung }\end{array}$ & $\begin{array}{l}\text { versteht einfache } \\
\text { Aufforderungen, } \\
\text { leichte } \\
\text { kontinuierliche } \\
\text { Fortschritte }\end{array}$ & $\begin{array}{l}\text { massiv } \\
\text { eingeschränkt }\end{array}$ \\
\hline $\begin{array}{l}\text { Verlauf } \\
\text { Sprach- } \\
\text { vermögen }\end{array}$ & guter Wortschatz & $\begin{array}{l}\text { verzögerte Ent- } \\
\text { wicklung, (6,9 J.): } \\
\text { Umfangreicher } \\
\text { Wortschatz bei } \\
\text { dysarthrischer } \\
\text { Sprache }\end{array}$ & $\begin{array}{l}\text { Sprachprodu- } \\
\text { ktion auf Vokale } \\
\text { beschränkt, } \\
\text { Sprachverständni } \\
\text { s ausgeprägter }\end{array}$ & $\begin{array}{l}\text { spricht einzelne } \\
\text { Wörter (3-5) }\end{array}$ & $\begin{array}{l}\text { keine Sprach- } \\
\text { produktion }\end{array}$ \\
\hline $\begin{array}{l}\text { Verlauf } \\
\text { Motorik, } \\
\text { Ataxie, } \\
\text { Muskeltonus }\end{array}$ & $\begin{array}{l}\text { verzögerte } \\
\text { Entwicklung, } \\
\text { freies Laufen mit } \\
24 \mathrm{M} \text {., rumpf- } \\
\text { betonte } \\
\text { Hypotonie, } \\
\text { Regression der } \\
\text { motorischen } \\
\text { Fähigkeiten }\end{array}$ & $\begin{array}{l}\text { Statomotorische } \\
\text { Retardierung u. } \\
\text { muskuläre } \\
\text { Hypotonie. } \\
\text { (15 M.): Freies } \\
\text { Sitzen. (2,5 J.): } \\
\text { Freies Stehen mit } \\
\text { Orthese. (5 J.): } \\
\text { Progredienter } \\
\text { Kraftverlust, } \\
\text { Tonuserhöhung. } \\
\text { (6,9 J.): } \\
\text { Tetraspastik }\end{array}$ & $\begin{array}{l}\text { ausgeprägte } \\
\text { motorische Ent- } \\
\text { wicklungs- } \\
\text { verzögerung mit } \\
\text { geringer Kopf- } \\
\text { und Rumpf- } \\
\text { kontrolle, } \\
\text { Paraspastik. } \\
\text { Zunahme der } \\
\text { Symptomatik mit } \\
\text { (4,1 J.). Freies } \\
\text { Sitzen o. Stehen } \\
\text { nicht möglich. }\end{array}$ & $\begin{array}{l}\text { deutliche } \\
\text { Entwicklungs- } \\
\text { Verzögerung, } \\
\text { muskuläre } \\
\text { Hypotonie, } \\
\text { Paraspastik, } \\
\text { schwache } \\
\text { Kopfkontrolle. } \\
\text { Freies Sitzen o. } \\
\text { Stehen mit 4,7 J. } \\
\text { nicht möglich. }\end{array}$ & $\begin{array}{l}\text { ausgeprägte } \\
\text { spastische } \\
\text { Tetraparese } \\
\text { (Nahrungs- } \\
\text { aufnahme über } \\
\text { Sonde) }\end{array}$ \\
\hline
\end{tabular}




\begin{tabular}{|c|c|c|c|c|c|}
\hline & $\begin{array}{l}\text { Patient } 3 \\
\text { (7,9 J.) }\end{array}$ & $\begin{array}{l}\text { Patient } 4 \\
(1,5 \mathrm{~J} . \text { bis } 6,9 \mathrm{~J} .)\end{array}$ & $\begin{array}{l}\text { Patient } 5 \\
(3,2 \text { J. bis } 4,1 \mathrm{~J} .)\end{array}$ & $\begin{array}{l}\text { Patient } 6 \\
(2,4 \text { bis } 4.7 \mathrm{~J} .)\end{array}$ & $\begin{array}{l}\text { Patient } 7 \\
(11,2 \mathrm{~J} .)\end{array}$ \\
\hline MRT & $\begin{array}{l}\text { Globale Hypo- } \\
\text { myelinisierung, } \\
\text { geringgradiges } \\
\text { Myelinsignal im } \\
\text { Forceps frontalis } \\
\text { und occipitalis, in } \\
\text { der Sehstrahlung } \\
\text { u. Pyramiden- } \\
\text { bahn (MRT } \\
\text { 7,9 J.) }\end{array}$ & $\begin{array}{l}\text { Globale } \\
\text { Myelinisierungs- } \\
\text { verzögerung, } \\
\text { (6,9 J.): Global } \\
\text { fleckförmig } \\
\text { zugenommene } \\
\text { Myelinisierung } \\
\text { bei insgesamt } \\
\text { ausgeprägter } \\
\text { Hypomyelini- } \\
\text { sierung }\end{array}$ & $\begin{array}{l}\text { Hypomyelini- } \\
\text { sierung, im } \\
\text { Verlauf gering- } \\
\text { gradiges Myelin- } \\
\text { signal in } \\
\text { C. Callosum u. } \\
\text { Pyramidenbahn. } \\
\text { (4,1 J.): Zunahme } \\
\text { der Myelini- } \\
\text { sierung bei } \\
\text { Myelinisierungs- } \\
\text { rückstand }\end{array}$ & $\begin{array}{l}\text { Hypomyelini- } \\
\text { sierung, im } \\
\text { Verlauf gering- } \\
\text { gradiges Myelin- } \\
\text { signal in der } \\
\text { Pyramidenbahn } \\
\text { und in den } \\
\text { Kleinhirnstielen }\end{array}$ & $\begin{array}{l}\text { Fehlende } \\
\text { Myelinisierung } \\
\text { und Hirn- } \\
\text { volumen- } \\
\text { minderung insb. } \\
\text { frontotemporal, } \\
\text { C. Callosum, } \\
\text { infratentoriell des } \\
\text { Mesencephalon, } \\
\text { in der Pons und } \\
\text { im Kleinhirn }\end{array}$ \\
\hline
\end{tabular}

ED: Erstdiagnose, J.: Jahre, kogn.: kongnitiv, M.: Monat, C. Callosum: Corpus Callosum *nicht genauer bekannt

Tabelle 5: Übersicht Erstdiagnose und MRT-Untersuchungen der PMS-Patienten

\begin{tabular}{llll}
\hline Patient & ED PMS & Alter erstes, letztes MRT & Anzahl MRT-Untersuchung \\
\hline Patient 3 & 7,9 J. & $7,9 \mathrm{~J}$. & 1 \\
Patient 4 & $1,3 \mathrm{~J}$. & $1,5 \mathrm{~J} ., 6,9 \mathrm{~J}$. & 2 \\
Patient 5 & $0,9 \mathrm{~J}$. & $3,2 \mathrm{~J} ., 4,4 \mathrm{~J}$. & 2 \\
Patient 6 & $0,8 \mathrm{~J}$. & $2,4 \mathrm{~J} ., 4,7 \mathrm{~J}$. & 4 \\
Patient 7 & $1,5 \mathrm{~J}$. & $11,2 \mathrm{~J}$. & 1 \\
\hline
\end{tabular}

ED: Erstdiagnose, J.: Jahre

\subsubsection{Klassifizierung PMS-Patienten}

Die Patienten mit PMS wurden mithilfe des Funktionsbeeinträchtigungs-Score (FDS) von Laukka et al. (2013) in die vier Gruppen PMS0, PMS1-2, PMS3 und PMS4 eingeteilt (s. Tabelle 6). Der FDS misst die Fähigkeit von Patienten Aufgaben des täglichen Lebens wie Nahrungsaufnahme, Waschen, Anziehen und Fortbewegung zu erfüllen (s. Tabelle 7). Die Interrater-Reliabilität dieses Bewertungssystems wird als $>95 \%$ angegeben (Laukka et al. 2014). Die Punktzahl der Patienten ist in der Tabelle 8 aufgeführt. Ergänzt wurde der Bereich „Kopfkontrolle“ und im Bereich Bildung die Items „reguläre Schulbildung, aber nicht in altersgemäßer Klassenstufe“ um „Kindergarten mit besonderer Betreuung“ und „Regelschule, altersgemäße Klassenstufe“ um „Besuch eines Regel-Kindergartens“ (s. Tabelle 7). Die Gruppe PMS0 umfasste Patient 3, die Gruppe PMS1-2 den Patient 4, die Gruppe PMS3 die Patienten 5 und 6 und die Gruppe PMS4 Patient 7. Die Gruppeneinteilung in PMS1-4 war adaptiert an Motorischen-Entwicklungs-Score (MDS) von Sarret et al. (2016). Diese verwendeten in ihrer PMS-DTI-Studie die MDS-Kategorien „PMS0: Keine motorischen Fähigkeiten“, „PMS1: Kopfkontrolle vorhanden“, „PMS2: Sitzposition möglich“, „PMS3: Gehen mit Unterstützung möglich“, „PMS4: Freies Gehen möglich“. In dieser Arbeit wurde aus Übersichtsgründen die umgekehrte Reihenfolge gewählt (PMS0: Milde Klinik, freies Gehen möglich, PMS1: Moderate Klinik, Gehen mit Unterstützung 
möglich, PMS2: Moderate Klinik, Sitzposition möglich, PMS3: Schwere Klinik, Kopfkontrolle vorhanden, PMS4: Sehr schwere Klinik, keine motorischen Fähigkeiten).

Tabelle 6: Klinische Einteilung der Patienten mit PMS

\begin{tabular}{lllllll}
\hline Gruppe & Klinische Symptomatik & FDS-Score & $\mathbf{n}_{\text {Patienten }}$ & $\mathbf{n}_{\text {Messungen }}$ & Altersspanne & MW + SD \\
\hline PMS0 & milde & $>20$ & 1 & 1 & $7,9 \mathrm{~J}$. & 7,9 \\
PMS1-2 & moderat & $16-20$ & 1 & 1 & $1,5-6,9 \mathrm{~J}$. & $4,3 \pm 3,7$ \\
PMS3 & schwer & $10-15$ & 2 & 6 & $2,4-4,7 \mathrm{~J}$. & $3,5 \pm 0,8$ \\
PMS4 & sehrschwer & $<10$ & 1 & 1 & $11,2 \mathrm{~J}$. & 11,2
\end{tabular}

Tabelle 7: Modifizierter Funktionsbeeinträchtigungs-Score

\begin{tabular}{|c|c|c|}
\hline Bereich & & Items \\
\hline \multirow[t]{4}{*}{ Bildung } & 0 & keine formelle Schulbildung \\
\hline & 1 & Förderschulunterricht \\
\hline & 2 & $\begin{array}{l}\text { reguläre Schulbildung, aber nicht in altersgemäßer Klassenstufe (Kindergarten mit } \\
\text { besonderer Betreuung, Ergänzung M.P.) }\end{array}$ \\
\hline & 3 & $\begin{array}{l}\text { Regelschule, altersgemäße Klassenstufe, (Besuch eines Regel-Kindergartens, Ergänzung } \\
\text { M.P.) }\end{array}$ \\
\hline \multirow[t]{5}{*}{ Sprache } & 0 & keine verbale Kommunikation \\
\hline & 1 & seltene verständliche Wörter, v.a. nonverbale Kommunikation \\
\hline & 2 & schwer verständliche Sprache \\
\hline & 3 & leicht verständliche Sprache, aber erkennbare Sprachstörung \\
\hline & 4 & keine erkennbare Sprachstörung \\
\hline \multirow{5}{*}{$\begin{array}{l}\text { Nahrungs- } \\
\text { aufnahme }\end{array}$} & 0 & ausschließlich Sondenernährung \\
\hline & 1 & teilweise orale Ernährung, mit zusätzlichen Sondenernährung \\
\hline & 2 & orale Fütterungen mit Konsistenzveränderungen in der Ernährung \\
\hline & 3 & normale Ernährung mit gelegentlichem Würgen \\
\hline & 4 & normales Schlucken der Nahrung möglich \\
\hline \multirow[t]{4}{*}{ Anziehen } & 0 & totale Abhängigkeit \\
\hline & 1 & kann beim Anziehen helfen, ist aber von anderen abhängig \\
\hline & 2 & unabhängig, aber mit vermindertem Wirkungsgrad \\
\hline & 3 & eigenständig \\
\hline \multirow[t]{4}{*}{ Toilettengang } & 0 & totale Abhängigkeit \\
\hline & 1 & benötigt Hilfe \\
\hline & 2 & unabhängig, aber mit vermindertem Wirkungsgrad \\
\hline & 3 & eigenständig \\
\hline \multirow[t]{4}{*}{ Schreiben } & 0 & totale Abhängigkeit \\
\hline & 1 & benötigt Hilfe \\
\hline & 2 & unabhängig, aber mit vermindertem Wirkungsgrad \\
\hline & 3 & eigenständig \\
\hline
\end{tabular}




\begin{tabular}{|c|c|c|}
\hline Bereich & & Items \\
\hline $\begin{array}{l}\text { Kopfkontrolle } \\
\text { (Ergänzung M.P.) }\end{array}$ & $\begin{array}{l}0 \\
1 \\
2\end{array}$ & $\begin{array}{l}\text { Keine Kopfkontrolle } \\
\text { Schwache Kopfkontrolle, in Bauchlage Kopfkontrolle } \\
\text { Gute Kopfkontrolle }\end{array}$ \\
\hline Sitzen & $\begin{array}{l}0 \\
1 \\
2\end{array}$ & $\begin{array}{l}\text { kann nicht ohne Unterstützung sitzen } \\
\text { gehaltenes Sitzen möglich } \\
\text { kann ohne Unterstützung sitzen }\end{array}$ \\
\hline Laufen & $\begin{array}{l}0 \\
1 \\
2 \\
3 \\
4 \\
5\end{array}$ & $\begin{array}{l}\text { Rollstuhl oder Bettgebunden } \\
\text { kann krabbeln } \\
\text { kann einige Schritte gehen, benötigt aber Hilfsmittel oder Unterstützung } \\
\text { benötigt für das Laufen von } 20 \mathrm{~m} \text { Hilfestellung } \\
\text { ataktischer Gang, aber Laufen ohne Hilfsmittel möglich } \\
\text { normaler Gang }\end{array}$ \\
\hline Atmung & $\begin{array}{l}0 \\
1 \\
2 \\
3\end{array}$ & $\begin{array}{l}\text { Beatmungsgerät oder konstante Atemunterstützung nötig } \\
\text { intermittierende Verwendung von nicht-invasiver Atemunterstützung } \\
\text { Atemschwierigkeiten, benötigt aber keine Unterstützung } \\
\text { normale Atmung }\end{array}$ \\
\hline
\end{tabular}

Quelle: Vgl. Laukka et al (2013), Übersetzung und Modifizierung: M. P.

Tabelle 8: Punktzahl der Patienten mit PMS im Funktionsbeeinträchtigungs-Score

\begin{tabular}{|c|c|c|c|c|c|c|c|c|c|c|}
\hline & $\begin{array}{l}\text { Pat } 3 \\
(7,9 \text { J. })\end{array}$ & $\begin{array}{l}\text { Pat } 4 \\
(1,5 \text { J.) }\end{array}$ & $\begin{array}{l}\text { Pat } 4 \\
(6,9 \text { J.) }\end{array}$ & $\begin{array}{l}\text { Pat } 5 \\
(3,2 \mathrm{~J} .)\end{array}$ & $\begin{array}{l}\text { Pat } 5 \\
(4,4 \text { J. })\end{array}$ & $\begin{array}{l}\text { Pat } 6 \\
(2,4 \mathrm{~J} .)\end{array}$ & $\begin{array}{l}\text { Pat } 6 \\
(2,8 \mathrm{~J} .)\end{array}$ & $\begin{array}{l}\text { Pat } 6 \\
(3,6 \mathrm{~J} .)\end{array}$ & $\begin{array}{l}\text { Pat } 6 \\
(4,7 \mathrm{~J} .)\end{array}$ & $\begin{array}{l}\text { Pat } 7 \\
(6,9 \mathrm{~J} .)\end{array}$ \\
\hline Bildung & 1 & $1^{*}$ & 1 & 2 & 2 & $1^{*}$ & $1 *$ & $1^{*}$ & 1 & 1 \\
\hline Sprache & 4 & 2 & 4 & 1 & 1 & 1 & 1 & $1,5^{* *}$ & $1,5^{* *}$ & 1 \\
\hline $\begin{array}{l}\text { Nahrungs- } \\
\text { aufnahme }\end{array}$ & 3 & 3 & 3 & 3 & 3 & 3 & 3 & 3 & 3 & 1 \\
\hline Anziehen & 2 & $1^{*}$ & 1 & 1 & 1 & $1^{*}$ & $1 *$ & $1^{*}$ & 1 & 0 \\
\hline $\begin{array}{l}\text { Toiletten- } \\
\text { gang }\end{array}$ & 2 & $1^{*}$ & 2 & 2 & 2 & 0 & 0 & 0 & 0 & 0 \\
\hline Schreiben & 2 & $1^{*}$ & 1 & $1^{*}$ & $1^{*}$ & $0^{*}$ & $0^{*}$ & $0^{*}$ & 0 & 0 \\
\hline $\begin{array}{l}\text { Kopf- } \\
\text { kontrolle }\end{array}$ & 2 & 2 & 2 & 1 & 1 & 0 & 1 & 1 & 1 & 0 \\
\hline Sitzen & 2 & 2 & 2 & 0 & 0 & 0 & 1 & 1 & 1 & 0 \\
\hline Laufen & 3 & 1 & 2 & 0 & 0 & 1 & 1 & 1 & 1 & 0 \\
\hline Atmung & 3 & 3 & 3 & 3 & 3 & 3 & 3 & 3 & 3 & 3 \\
\hline Gesamt & 24 & $17^{*}$ & 19 & $14^{*}$ & $14^{*}$ & 10 & 12 & 12,5 & 12,5 & 6 \\
\hline
\end{tabular}

Pat: Patient. * Nicht adäquat bestimmbar. ** 0,5 Punkte für Besserung des Sprachverständnisses 


\subsubsection{Zerebrale-Folatdefizienz}

Die dritte Patientengruppe umfasste fünf Patienten mit cFTD und HFM. Das Alter der Patienten war zwischen drei Monaten und 12 Jahren (s. Tabelle 9, 10 und 11). Die Patienten 8, 9 und 10 sind Geschwister. Sie wiesen wie auch die Patientin 11 eine FOLR1-Mutation auf. Bei Patient 12 lag eine Mutation im SLC46A1-Gen vor, die zur HFM mit peripherer und zerebraler Folatdefizienz führt (s. Tabelle 11).

Tabelle 9: Übersicht I der Klinik, Diagnostik und Therapie der Patienten mit cFD

\begin{tabular}{|c|c|c|c|}
\hline & Patient 8 (3,8 J. - 12,0 J.) & Patientin 9 (2,3 J. - 11,1 J.) & Patientin 10 (0,2 J. - 7,0 J.) \\
\hline $\begin{array}{l}\text { Genetische } \\
\text { Veränderung }\end{array}$ & $\begin{array}{l}\text { FOLR1 } \\
\text { p.Q118X, p.C175X) } \\
\text { (nonsense) }\end{array}$ & $\begin{array}{l}\text { FOLR1 } \\
\text { p.Q118X, p.C175X } \\
\text { (nonsense) }\end{array}$ & $\begin{array}{l}\text { FOLR1 } \\
\text { p.Q118X, p.C175X } \\
\text { (nonsense) }\end{array}$ \\
\hline Erkrankung & cFTD & cFTD & cFTD \\
\hline Familienanamnese & $\begin{array}{l}\text { beide Geschwister ebenfalls } \\
\text { betroffen }\end{array}$ & $\begin{array}{l}\text { beide Geschwister ebenfalls } \\
\text { betroffen }\end{array}$ & $\begin{array}{l}\text { beide Geschwister ebenfalls } \\
\text { betroffen }\end{array}$ \\
\hline $\begin{array}{l}\text { Beginn erste } \\
\text { Symptome }\end{array}$ & circa $2,5 \mathrm{~J}$ & $\operatorname{circa} 2,3 \mathrm{~J}$ & keine \\
\hline Verlauf & zunächst adäquate & unauffällige & unauffällige \\
\hline Kognition & $\begin{array}{l}\text { Entwicklung, mit 2,5 J } \\
\text { langsame Regression. Ab } \\
5 \text { J. unter Therapie } \\
\text { deutliche Besserung: } \\
\text { Mit } 12 \text { J. Kontakt möglich, } \\
\text { soziales Lächeln. Autistische } \\
\text { Verhaltenszüge }\end{array}$ & Entwicklung & Entwicklung \\
\hline$\frac{\text { Verlauf }}{\text { Sprachvermögen }}$ & $\begin{array}{l}\text { Verlust mit } 3 \text { J., } \\
(12,0 \text { J.): Laute }\end{array}$ & altersgerecht & altersgerecht \\
\hline $\begin{array}{l}\text { Verlauf } \\
\text { Motorik/ } \\
\text { Muskeltonus/ } \\
\text { Bewegungs- } \\
\text { störungen / } \\
\text { Koordination }\end{array}$ & $\begin{array}{l}\text { (4 J.) Rumpfhypotonie u. } \\
\text { spastische Tetraparese } \\
\text { Ataxie, Athetose, } \\
\text { Myoklonie, Tremor, } \\
\text { Hyporeflexie. } \\
\text { (12,0 J.): Normaler } \\
\text { Muskeltonus, leichte } \\
\text { Schwäche in den } \\
\text { Nackenmuskeln, freies } \\
\text { Stehen und Laufen möglich } \\
\text { klobiger Gang, inter- } \\
\text { mittierend im Rollstuhl. } \\
\text { Ataxie, Rumpfhypotonie } \\
\text { rückläufig }\end{array}$ & $\begin{array}{l}\text { (2,3 J.) Fallneigung u. Gang- } \\
\text { unsicherheit, Ataxie. Unter } \\
\text { Therapie bis zum Alter von } \\
\text { 4 J. komplett } \\
\text { zurückentwickelt. } \\
\text { Meilensteine wurden } \\
\text { altersgerecht erreicht }\end{array}$ & altersgerecht \\
\hline
\end{tabular}




\begin{tabular}{|c|c|c|c|}
\hline & Patient 8 (3,8 J. - 12,0 J.) & Patientin $9(2,3 \mathrm{~J} .-11,1 \mathrm{~J})$. & Patientin 10 (0,2 J. - 7,0 J.) \\
\hline Krampfanfälle & $\begin{array}{l}\text { zunächst bis } \underline{50 x} \text { täglich, } \\
\text { unter Therapie rückläufig, } \\
\text { seit } 11 . \text { Lj. wieder häufiger } \\
\text { (1-3x tägl.) }\end{array}$ & keine & keine \\
\hline $\begin{array}{l}\text { 5-MTHF- } \\
\text { Konzentration im } \\
\text { CSF }\end{array}$ & $\begin{array}{l}\text { vor Therapie (3,8J.) } 1,4 \\
\text { nmol/L (Referenz: } 43-159 \\
\text { nmol/L) }\end{array}$ & $\begin{array}{l}\text { vor Therapie (2,3J.) } 5 \text { - } \\
\text { MTHF unterhalb der } \\
\text { Nachweisgrenze }\end{array}$ & durchweg normwertig \\
\hline \multirow[t]{2}{*}{$\begin{array}{l}\text { Konventionelles } \\
\text { MRT }\end{array}$} & $\begin{array}{l}\text { ( } 3 \mathrm{~J} .): \text { Hypomyelinisierung, } \\
\text { globale Atrophie insb. im }\end{array}$ & $\begin{array}{l}\text { (3 J.): Hypomyelinisierung, } \\
\text { Kleinhirnatrophie }\end{array}$ & $\begin{array}{l}\text { altersentsprechende und } \\
\text { zeitgerechte Myelinisierung }\end{array}$ \\
\hline & $\begin{array}{l}\text { Kleinhirn, permanente T2- } \\
\text { hyperintense WM-Läsionen. } \\
\text { (12 J.): Deutliche Zunahme } \\
\text { der Myelinisierung im } \\
\text { Großhirn. Kleinhirnatrophie } \\
\text { unverändert }\end{array}$ & $\begin{array}{l}\text { (11 J.): Beinahe vollständige } \\
\text { Myelinisierung, weiterhin } \\
\text { leichte zerebelläre Atrophie }\end{array}$ & \\
\hline Therapie & $\begin{array}{l}\text { p.o.(3,8 J.)/i.v. }(4,9 \mathrm{~J} .) / \text { i.th. } \\
\text { (5,6 J.) Substitution mit } \\
\text { Folinsäure*, Antikonvulsiva } \\
\text { (Valproat, Levitiracetam) }\end{array}$ & $\begin{array}{l}\text { p.o. }(2,3 \mathrm{~J} .) \text { i.v. }(3,9 \mathrm{~J} .) \\
\text { Substitution mit Folinsäure* }\end{array}$ & $\begin{array}{l}\text { p.o. }(0,2 \mathrm{~J} .) \text { Substitution mit } \\
\text { Folinsäure* }\end{array}$ \\
\hline
\end{tabular}

Lj.: Lebensjahr. J: Jahre. *Das Alter gibt den Zeitpunkt der Initiation der Therapie an

Tabelle 10: Übersicht II der Klinik, Diagnostik und Therapie der Patienten mit cFD

\begin{tabular}{lll}
\hline & Patientin 11 (5,0 J. bis 7,0 J.) & Patient 12 (1,3 J. bis 6,2 J.) \\
\hline Genetische Veränderung & $\begin{array}{l}\text { FOLR1 } \\
\text { c.240C }>\text { G; p. Tyr80 } \\
\text { (homozygote Stopmutation) }\end{array}$ & $\begin{array}{l}S L C 46 A 1 \\
\text { c.781G>T, p.E261 } \\
\text { (homozygote missense) }\end{array}$ \\
Erkrankung & cFTD & HFM \\
Familienamnese & keine Geschwister & \\
& & $\begin{array}{l}\text { zwei Geschwister mit drei Monaten an } \\
\text { pulmonalen Infekten verstorben, ein } \\
\text { gesunder älterer Bruder }\end{array}$ \\
Beginn erste Symptome & circa 2,5 J. &
\end{tabular}

${ }^{2}$ Abweichend Normwerte in unterschiedlichen Quellen: 2-5 J.: 43 - $159 \mathrm{nmol} / \mathrm{L}$ (median: $74 \mathrm{nmol} / \mathrm{L}$ ) (Verbeek et al. (2008); 2-3J.: 44-122 nmol/L (median: $72 \mathrm{nmol} / \mathrm{L}), 4-18 \mathrm{~J} .: 42-81 \mathrm{nmol} / \mathrm{L}$ (median: $56 \mathrm{nmol} / \mathrm{L}$ ) (Ormazabal et al. 2006); 11M.-4J.: 48-127 nmol/L (median: $80 \mathrm{nmol} / \mathrm{L}$ ), 5-18 J.: 35-124 nmol/L (median: $66 \mathrm{nmol} / \mathrm{L}$ ) (Pérez-Dueñas et al. 2011) 


\section{Verlauf}

Kognition

Verlauf

Sprachvermögen ab 2,5 J. Regression kognitiver

Fähigkeiten,

(7,7 J.): Besucht aktuell Förderschule, geringer u. sehr langsamer Lernfortschritt

Regression des Sprachvermögen ab 2,5 J. $(7.0 \mathrm{~J})$ : Sprache verlangsamt bei stark reduziertem Wortschatz

Ab 2,5 Lj. Regression der motorischen Fähigkeiten, Ataxie, Dysmetrie.

(5,5 J.): zunächst ausgeprägte Ataxie, unter Therapie fast vollständig rückläufig

(7,7 J.): Sicheres Gangbild, leicht bestehender Tremor.

Multiple myoklonale Anfälle (Beginn 5,2 J.) bis zu 5-mal/Tag. Unter antikonvulsiver Therapie im Verlauf weitgehend anfallsfrei

vor Therapie $(<5 \mathrm{nmol} / \mathrm{L})$

\section{5-MTHF-Konzentration} im CSF

\section{Konventionelles MRT}

Therapie
(5,0 J.): Frontal und zerebellär betonte Atrophie, Hypomyelinisierung $(7,0)$ : Minimale Hirnvolumenminderung der WM im Vergleich zur altersgerechten Kontrolle, regelrechte Mark-RindenDifferenzierbarkeit und unauffällige Konfiguration des Kleinhirns altersentsprechende intellektuelle Leistungen

initial expressive Sprachstörung.

(5,7 J.): Altersgerechter Stand

(1,3 J.): Leichte Ataxie. Unter Therapie vollständig rückläufig

(6,2 J.): leichte Koordinations- und Feinmotorik-Schwierigkeiten, unter Therapie vollständig rückläufig

Residuum leicht breitbasiges Gangbild

$(1,3 \mathrm{~J}$.) Insgesamt drei fiebergebundene Krampfanfälle

(0,3 J.): Normales 5-MTHF im CSF, im Serum deutlich reduziert. (1,3 J.): 5MTHF im CSF unterhalb Nachweisgrenze

(1,3 J.): Hypomyelinisierung. Läsionen beidseitig in parieto-okzipitaler und subkortikaler WM. Geringfügige Aufweitung der äußeren Hirnfurchen frontal und zerebellär. (6,2 J.): deutliche Zunahme der Myelinisierung. Bilaterale, parieto-okzipitale flächige T1-hypointense

und T2-hyperintense Marklagerveränderungen, unvollständige Myelinisierung beidseits temporal

p.o. $(1,3 \mathrm{~J}$.$) / i.v. (1,3 \mathrm{~J}$.$) / i.th. (1,8 \mathrm{~J}$.)

Folinsäure Substitution*
Substitution mit Folinsäure*

Antikonvulsive Therapie (Levitiracetam) 
Tabelle 11: Übersicht Erstdiagnose und MRT-Untersuchungen der Patienten mit cFD

\begin{tabular}{llll}
\hline Patient & ED cFD & Alter erstes, letztes MRT & Anzahl MRT-Untersuchungen \\
\hline Patient 8 & 3,8 J. & 3,8 J., 12,0 J. & 12 \\
Patientin 9 & 2,3 J. & 2,3 J., 11,1 J. & 11 \\
Patientin 10 & 0,2 J. & 0,2 J., 8,1 J. & 10 \\
Patientin 11 & 5,0 J. & 5,0 J., 7,0 J. & 5 \\
Patient 12 & 1,3 J. & 1,3 J., 6,2 J. & 10 \\
\hline
\end{tabular}

ED: Erstdiagnose, J.: Jahre

\subsection{Aufnahmetechnik und Durchführung}

\subsubsection{Aufnahmegerät und Sequenzen der Magnetresonanztomographie}

Die MRT-Untersuchungen wurden mit einem 3-Tesla-MR-Tomographen (Magnetom TrioTim syngo MR B15, Siemens Medical Solutions, Erlangen, Deutschland) mit einer 8-Kanal-phase-array-Empfangsspule im Forschungszentrum MR-Forschung in der Neurologie und Psychiatrie des Instituts für Kognitive Neurologie der Universitätsmedizin Göttingen zwischen November 2005 und April 2018 durchgeführt. Die Aufnahmen umfassten als konventionell-diagnostische Sequenzen eine T1-gewichtete Gradientenechosequenz (MP-RAGE) mit TR/TI/TE = 2250/900/3,26 ms (7,5 Minuten Messzeit) und eine T2-gewichtete Turbo-Spin-Echo-Sequenz (TSE) mit TR/TE= 2900/425 ms (4,5 Minuten Messzeit) (s. Tabelle 12).

Tabelle 12: Überblick konventionell-diagnostische und myelinsensitive MRT-Sequenzen

\begin{tabular}{lllll}
\hline & TR/TE [ms] & Schichtanzahl/ -dicke & FOV [mm2] & Voxel [mm3] \\
\hline T1 MP-RAGE & $2250 / 3,26$ & $176 / 1 \mathrm{~mm}$ & 256 & 1 \\
TSE & $2900 / 425$ & $144 / 1 \mathrm{~mm}$ & 240 & 0,8 \\
PD_MTflash & $25 / 4,92$ & $128 / 1,25 \mathrm{~mm}$ & 240 & 2,2 \\
MT_MTflash & $25 / 4,92$ & $128 / 1,25 \mathrm{~mm}$ & 240 & 2,2 \\
T1_MTflash & $11 / 4,92$ & $128 / 1,25 \mathrm{~mm}$ & 240 & 2,2 \\
RFmap_TSTEAM_60 & $19400 / 7,54$ & $45 / 2,9 \mathrm{~mm}$ & 224 & 35,5 \\
RFmap_TSTEAM_100 & $19400 / 7,54$ & $45 / 2,9 \mathrm{~mm}$ & 224 & 35,5 \\
T-STEAM_diff & $15440 / 7,08$ & $46 / 2,2 \mathrm{~mm}$ & 229 & 10,6 \\
ssSE-EPI & $7500 / 82$ & $64 / 2 \mathrm{~mm}$ & 208 & 8 \\
\hline
\end{tabular}

PD_mtflash: Protonendichte-gewichtete Magnetisierungstransfer-fast-low-angle-shot-Sequenz. MT_mtflash: Magnetisierungstransfer-fast-low-angle-shot-Sequenz. RFmap: Radiofrequenz Sequenz. STEAM: Stimulated-echo-acquisition-mode. sSE-EPI: single-shot-spin-echo-echoplanar imaging-Sequenz 


\subsubsection{Diffusionstensor-Bildgebung}

Die DT-Daten wurden zum einen mit einer STEAM-Sequenz (46 Schichten, 2,2 mm Dicke, 24 Diffusionsrichtungen bei $\mathrm{b}=950 \mathrm{~s} / \mathrm{mm}^{2}, \mathrm{~b}=0 \mathrm{~s} / \mathrm{mm}^{2}$ ), zum anderen mit einer EPISequenz (64 Schichten, $2 \mathrm{~mm}$ Dicke, 64 Diffusionsrichtungen bei $\mathrm{b}=950 \mathrm{~s} / \mathrm{mm}^{2}$, $\mathrm{b}=0 \mathrm{~s} / \mathrm{mm}^{2}$ ) erhoben (s. Tabelle 12). Für die Aufnahme der STEAM-Sequenzen wurden zwei unterschiedliche Richtungsprotokolle verwendet (STEAM_P1 und STEAM_P2). Das STEAM-Richtungsprotokoll STEAM_P1 wurde in der Abteilung MR-Forschung der Universitätsmedizin Göttingen bis Juni 2008 genutzt, das Protokoll STEAM_P2 seit Juli 2008. Insgesamt wurden drei STEAM-Aufnahmen durchgeführt. Zur Verbesserung des Signal-Rausch-Verhältnisses (SNR, signal to noise ratio) wurden diese hinterher gemittelt.

\subsubsection{Magnetisierungstransfer-Bildgebung}

Als Sequenz für die MT-Bildgebung wurde eine Fast-low-angle-shot (FLASH)-Sequenz (1,25 $\mathrm{mm}^{3}$ isotrope Auflösung, $240 \mathrm{~mm}$ field of view) verwendet (s. Tabelle 12). Der MTKontrast wurde auf einer PD-w Referenzkarte (TR/TE/ $\alpha=25 / 4,92 \mathrm{~ms} / 5^{\circ}$, Messzeit 3,5 Minuten) durch Einstellung eines $12,8 \mathrm{~ms}$ Gaußschen MT-Impulses erzeugt (540 ${ }^{\circ}$ nominaler Kippwinkel, 2,2 kHz Off-Resonanz). Die prozentuale MTsat wurde mithilfe einer zweiten T1-w-Referenzaufnahme berechnet (TR/ $\alpha=11 \mathrm{~ms} / 15^{\circ}$, Messzeit 1,5 min). Die gesamte Messzeit betrug 8,5 Minuten.

\subsubsection{Datenweiterverarbeitung}

\subsubsection{Diffusionsgewichtete Daten}

Die diffusionsgewichteten Datensätze im DICOM-Format wurden mit Diffusion Toolkit, einem Zusatzprogramm von TrackVis ${ }^{3}$, rekonstruiert. Das TrackVis eigene Tool erzeugte aus einem DTI-Datensatz eine graustufige Fraktionale Anisotropie (FA)-Karte, eine farbkodierte FA-Karte (ccFA, color-coded FA-Karte), sowie die Eigenwert-Karten E1 ( $\lambda 1$ ), E2

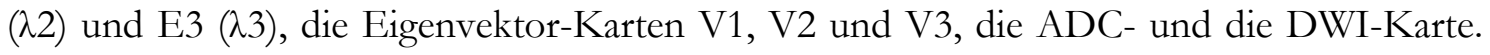
Die visuelle Darstellung der Karten erfolgte in TrackVis.

Die FA beschreibt die Gerichtetheit der Diffusion und ist ein errechneter Wert aus der Standardabweichung (SD, standard deviation) der Eigenwerte dividiert durch den Mittelwert der Eigenwertquadrate. Der Wert FA=1 entspricht einer hundertprozentigen Anisotropie, also der Diffusion von Wassermolekülen in ausschließlich eine Richtung. Die rote Farbkodierung der ccFA-Karte zeigt die Hauptdiffusion in Richtung links/rechts an. Die grüne Kolorierung entspricht einer Hauptdiffusion in Richtung anterior/posterior und die blaue einer Diffusion in Richtung superior/inferior (s. Abb. 3). Die Höhe der FA spiegelt

\footnotetext{
${ }^{3}$ Department of Radiology Massachusetts General Hospital; http://www.trackvis.org
} 
sich in der ccFA-Karte in der Farbintensivität wider. Die AD wird von dem größten Eigenwert $(\lambda 1)$ abgeleitet und gibt die Richtung der schnellsten Diffusion an. Sie spiegelt die Diffusion longitudinal zur Nervenfaser wider. Die RD ist die Mittelung der zweit- und drittgrößten Eigenwerte ( $\lambda 2$ und $\lambda 3$ ). Sie zeigt die Diffusion senkrecht zur Faserrichtung an. Die MD ist die Mittelung der drei Eigenwerte und gibt die molekulare, mittlere Diffusion in einem Voxel an.

\subsubsection{Magnetisierungstransfer-Daten}

Die archivierten DICOM-Bilder wurden mithilfe des Programms MRIcro ${ }^{4}$ zunächst zu dreidimensionalen Volumendaten im Analyze-Format zusammengefügt. Die weitere Bearbeitung erfolgte mithilfe von FSL 4.1 ${ }^{5}$. Mit FSL 4.1 und dessen Hilfsprogrammen Brain Extraction Tool und FSL Linear Registration Tool wurde zunächst die Segmentierung des Hirngewebes aus dem Neuro- und Viszerokranium durchgeführt sowie die anatomischen Datensätze über die interkommisurale Linie koregistriert. Die Kalkulation der MTsat erfolgte anschließend durch eine intern etablierte und publizierte Analyseroutine aus den Datensätzen PD-FLASH, MT-FLASH und T1-FLASH (Helms et al. 2008). In der erstellten MTsat-Karte wurden die MTsat-Werte farblich kodiert. Dazu wurde eine Cyan-Blau-Grau-Rot-GelbFarbskala mit dem Bereich -0,1 percent units (p. u.) (zyan: CSF) über 1,2 p. u. (grau: GM) bis 2,5 p. u. (gelb: myelinisierte WM) gewählt (s. Abb. 2). Zur besseren Darstellung der myelinisierten WM wurden der gelbe Schwellenwert der MTsat auf 2,5 p. u. gesenkt. Bei gesunden Probanden sind MTsat-Werte in der myelinisierten WM um 3 p. u. zu erwarten. Mit Herabsetzung des Schwellenwertes auf 2,5 p. u. wurde die Sensitivität für die Darstellung der WM erhöht (Dreha-Kulaczewski et al. 2012). In FSL View konnten die farbkodierte MTsat-Karte auf einer T1-w Referenzkarte angezeigt werden.

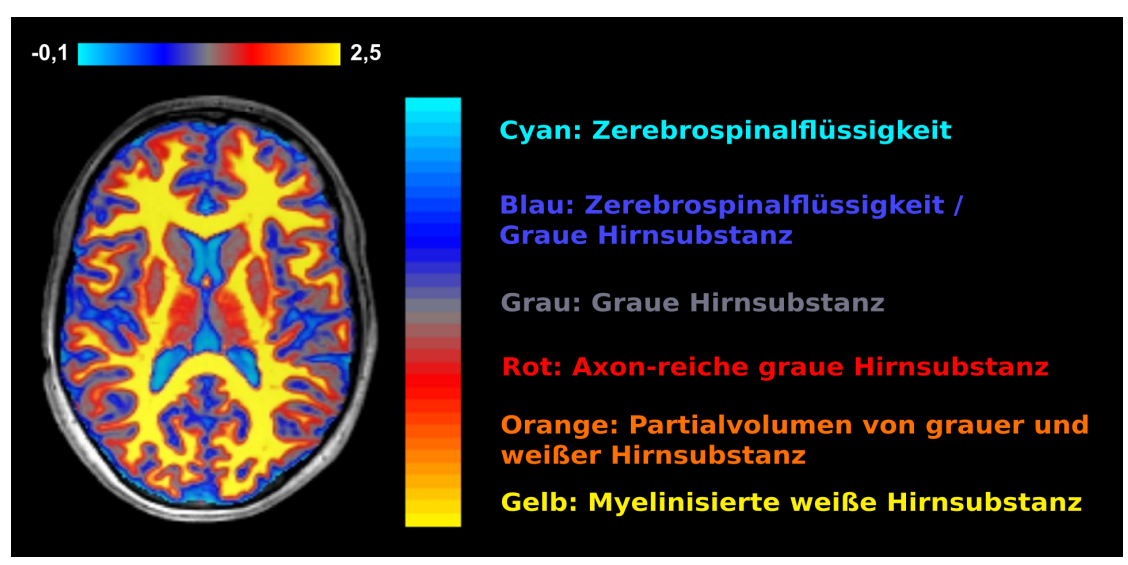

Abb. 2: Farbkolorierung der MTsat-Karten

Mit freundlicher Genehmigung von PD Dr. med. Dreha-Kulaczewski

\footnotetext{
${ }^{4}$ McCausland Center for Brain Imaging

${ }^{5}$ Oxford Centre for Functional MRI of the Brain
} 


\subsubsection{Region-of-interest-Analyse}

Die Region-of-interest (ROI)-Analyse erfolgte durch die manuelle a-priori Einzeichnung in den der folgenden Tabelle zu entnehmenden Hirnregionen (s. Tabelle 13).

Tabelle 13: Lokalisationen der Region-of-interest nach Patientengruppen

\begin{tabular}{lllll}
\hline & X-ALD & PMS & cFD & Kontrolle \\
\hline Genu & $\mathrm{X}$ & $\mathrm{X}$ & $\mathrm{X}$ & $\mathrm{X}$ \\
SCC & $\mathrm{X}$ & $\mathrm{X}$ & $\mathrm{X}$ & $\mathrm{X}$ \\
SCC superior & $\mathrm{X}$ & & & \\
SCC inferior & $\mathrm{X}$ & & & \\
SCC Läsion & $\mathrm{X}$ & & $\mathrm{X}$ & \\
CC & & $\mathrm{X}$ & $\mathrm{X}$ \\
Pyr & & $\mathrm{X}$ & $\mathrm{X}$ \\
WMLF & $\mathrm{X}$ & $\mathrm{X}$ & $\mathrm{X}$ & $\mathrm{X}$ \\
WMLPO & $\mathrm{X}$ & & $\mathrm{X}$ & $\mathrm{X}$ \\
\hline
\end{tabular}

GCC: Genu. SCC: Splenium. CC: Corpus Callosum. Pyr: Pyramidenbahn. WMLF: Links frontale weiße Hirnsubstanz. WMLPO: Links parieto-okzipitale weiße Hirnsubstanz

\subsubsection{Bestimmung der ROI in den diffusionsgewichteten Daten}

Die ROI wurden in den ccFA-Karten entlang der anatomischen Strukturen eingezeichnet. Dieses Verfahren ist in der Literatur anerkannt und beschrieben (Choudhri et al. 2014). Eine Karte mit einfacher Kolorierung der Voxel nach Hauptdiffusionsrichtung ohne Unterlegung der FA war nicht im Umfang der verwendete DTI-Software TrackVis. Die ROI in Genu, Splenium und im gesamten Corpus Callosum wurden in der Mittsagittale gesetzt (s. Abb. 3). Zur Demaskierung einzelner Artefakte wurde neben den mittsagittalen ROI zwei weitere ROI in der parasagittalen Schicht links und rechts der Mittsagittale gesetzt. Die einzelnen DT-Datenwerte wurden anschließend aus den drei ROI Links, Mitte, Rechts gemittelt. Zur präzisen Darstellung des Splenium bei den Patienten mit ccALD wurde das ROI Splenium in Splenium superior und Splenium inferior unterteilt. Dafür wurde eine Horizontale im Splenium gezogen (s. Abb. 3, b). Ziel war es den „Neutralisierungseffekt“ der abweichenden DTI-Werte in der Läsion durch die physiologischen Werte in den Voxel um die Läsion zu verringern. Für Lokalisation und Größe des Areals der Läsion (SCC Läsion) wurde als Referenz die ccFA zum Zeitpunkt der erstmaligen radiologischen Beschreibung der Läsion bei den zwei Patienten mit ccALD gewählt. Retrospektiv durch Übereinanderlegen der Aufnahmen und Vergleich der Lokalisationen versucht, das gleiche Areal in vorherigen Messungen auszuwählen.

Zum leichteren Verständnis wurde im Zusammenhang mit der ROI im Splenium und der Erkrankung ccALD in dieser Arbeit die Bezeichnung Gesamt-Splenium verwendet. Die ROI Corpus Callosum beinhaltet die Anteile des kompletten Corpus Callosum, also einschließlich Rostrum corporis callosi (in der Folge Rostrum), Genu, Truncus corporis callosi (in der Folge Truncus) und Splenium. Die ROI in den Pyramidenbahnen wurden in der Transversalebene 
eingezeichnet. Es wurde je eine ROI in der linken und in der rechten Pyramidenbahn platziert (s. Abb. 3). Die Mittelung der DT-Datenwerte der beiden ROI ergab den endgültigen Wert. Die in der ccFA-Karte eingezeichneten ROI wurden auf allen eingefügten Karten (FA, E1, E2, E3, V1, V2, V3) angezeigt. Die absoluten FA-Werte und die Eigenwerte in den ROI konnten TrackVis anschließend entnommen und in einer Exceltabelle dokumentiert werden. Zur Übersicht wurden in dieser Arbeit die Eigenwert-Werte mit $10^{3}$ multipliziert. Die AD, RD und MD sind somit nachfolgend in $10^{-3} \mathrm{~mm}^{2} \cdot \mathrm{s}^{-1}$ angegeben.

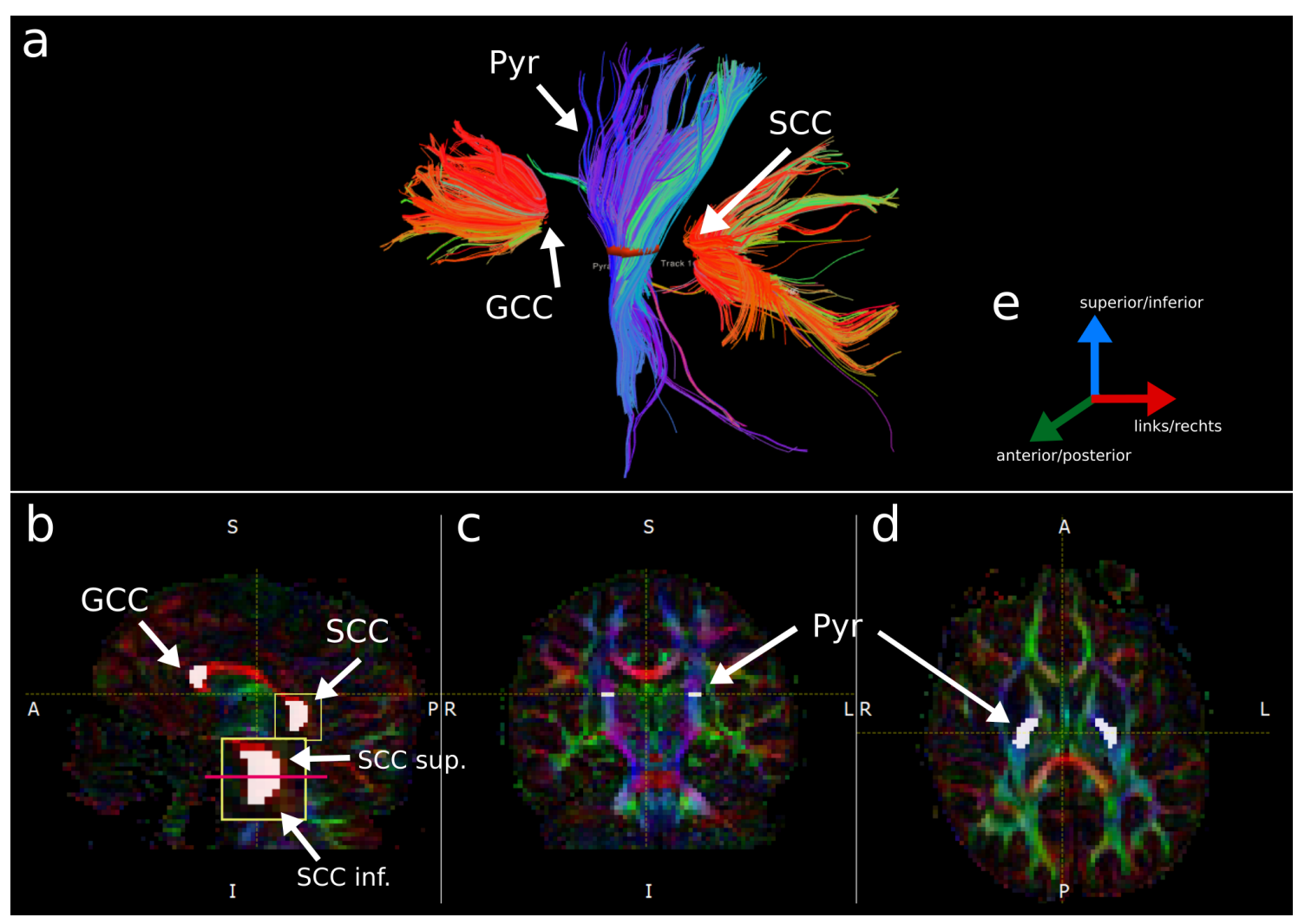

Abb. 3: ROI-Bestimmung in TrackVis

a: Traktographie der unter b,c,d eingezeichneten ROI b: ROI in Genu (GCC), Splenium (SCC), Splenium superior (SCC sup.) und inferior (SCC inf.) in der Sagitalebene c: ROI in der Pyramidenbahn (Pyr) in der Frontalabene d: ROI in der Pyramidenbahn in der Transversalebene e: Farbkodierung der Hauptdiffusionsrichtung. Rot: Haupdiffusionsrichtung links/rechts. Grün: Hauptdiffusionsrichtung anterior/posterior. Blau: Haupdiffusionsrichtung superior/inferior. Richtungsangaben: A: anterior P: posterior S: superior I: inferior L: Links R: Rechts

\subsubsection{Bestimmung der ROI in den Magnetisierungstransfer-Daten}

Die ROI-Analyse in den MTsat-Karten wurde mit Hilfe von MRIcro in den Regionen Genu, Splenium, links frontale WM (WMLF, left frontal white matter) und links parieto-okzpitale WM (WMLPO, left parieto occpitale white matter) durchgeführt. Die Setzung der ROI in Genu und Splenium erfolgte in der sagittalen koregistrierten T1-w Aufnahme (s. Abb. 4). Die Größe der ROI war abhängig von der anatomischen Struktur. In der Patientengruppe X-ALD wurden vergleichbar mit der ROI-Einzeichnung in den DTI-Daten zwei ROI im Splenium superior und inferior in der T1-w Aufnahme in MRIcro platziert (s. Abb. 4). Das Corpus Callosum war bei einigen PMS- und cFD-Patienten schmaler als bei den ccALD-Patienten. 
Aus diesem Grund wurde der Durchmesser der ROI in Genu und Splenium bei diesen Patienten etwas kleiner gewählt. In der WMLF wurde die ROI in der axialen Ebene in Höhe des mittleren Genu gesetzt. Die ROI wurde in der Mitte einer Linie zwischen dem Beginn der GM und dem Cornu frontale des Ventriculus lateralis eingezeichnet (s. Abb. 4). Die ROI in der WMLPO wurde in die Mitte einer Linie zwischen Beginn der GM und dem Cornu posterior des Ventriculus lateralis gesetzt (s. Abb. 4). Nach Platzierung der ROI auf den anatomischen T1-w Karten wurden die ROI auf die MTsat-Karten in MRIcro übertragen und der mittlere MTsat-Wert sowie die SD abgelesen. Für Lokalisation und Größe des Areals der Läsion (SCC Läsion) wurde als Referenz die MTsat-Karte zum Zeitpunkt der erstmaligen radiologischen Beschreibung der Läsion bei den zwei Patienten mit ccALD gewählt.

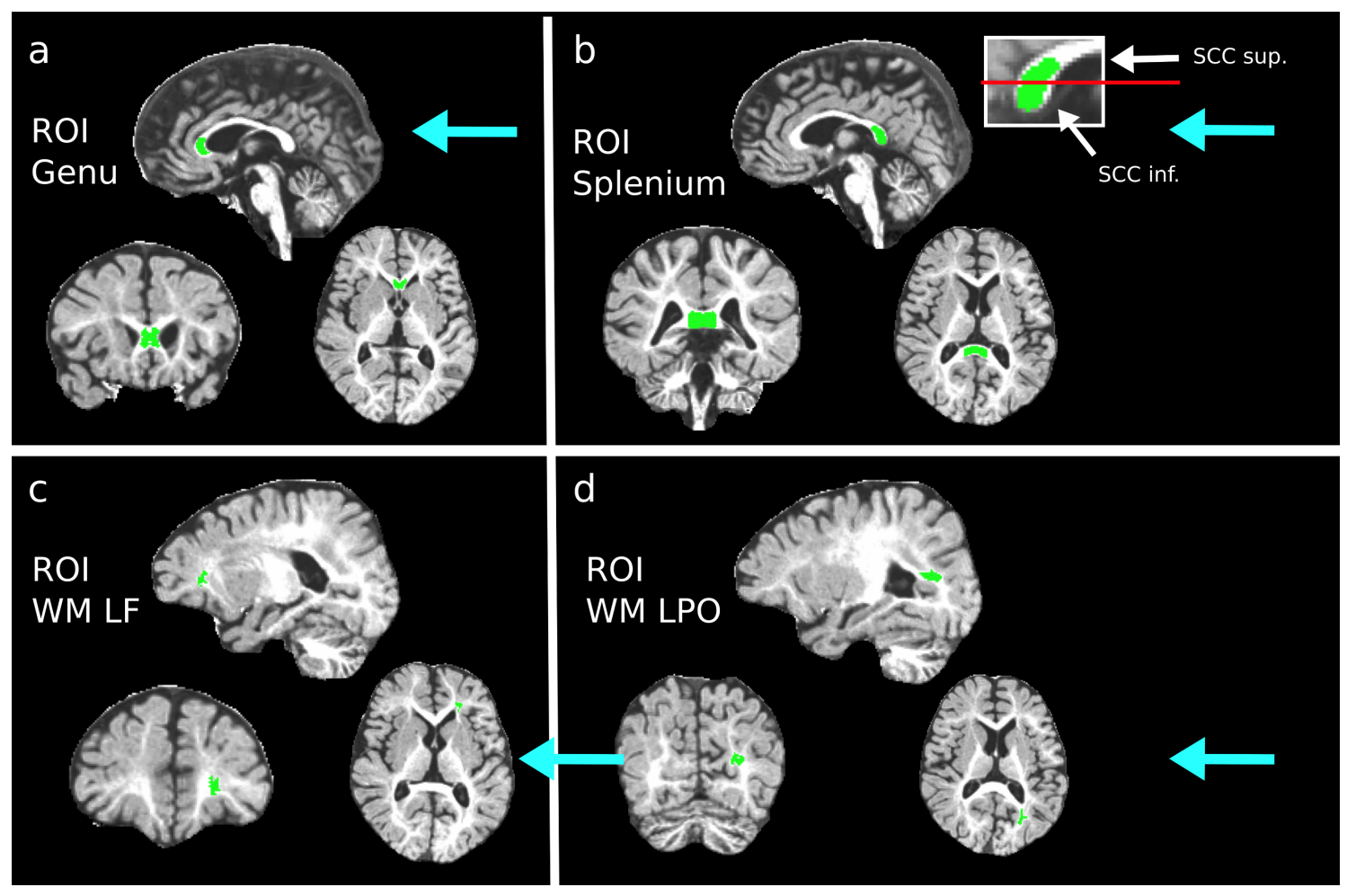

Abb. 4: ROI-Bestimmung in MRIcro

ROI (hellgrün) in den Lokalisationen Genu (a), Splenium (b), WMLF (c) und WMLPO (d). Blaue Pfeile: Ebenen, in denen die ROI eingezeichnet wurden. Weißer Kasten in (b): Vergrößerung des Splenium mit den ROI Splenium superior (SCC sup.) und Splenium inferior (SCC inf.) 


\section{Ergebnisse}

\subsection{Kontrollgruppe}

\subsubsection{Verlaufsbeschreibung der Myelinparameter der Kontrollgruppe}

\subsubsection{Qualitative Beurteilung der MRT-Aufnahmen}

In den konventionellen, qualitativen MRT-Aufnahmen zeigte sich makroskopisch bei den gesunden Probanden eine physiologisch zunehmende Myelinisierung zwischen dem ersten und fünftem Lebensjahr (s. Abb. 5). In den T1-w Aufnahmen imponierte die WM mit dem Alter zunehmend hyperintenser, in den T2-w Aufnahme zunehmend hypointenser. Die farbkodierten MTsat-Karten illustrierten die Zunahme der myelinisierten WM durch die Zunahme der gelben Areale (MTsat-Werte > 2,5 p. u.).

Bei der eineinhalb Jahre alten Kontrolle erschien die Myelinisierung im Bereich des Corpus Callosum und der Capsula interna abgeschlossen (Abb. 5). In den konventionellen und den quantitativen Karten der Patienten war zwischen dem ersten und dritten Lebensjahr eine Zunahme des Umfangs der kortikospinalen Bahnen zu erkennen (s. Abb. 5). Die Aufnahmen der Probanden, die älter als fünf Jahre waren, zeigten in der visuellen Betrachtung im Verlauf weder in den konventionellen noch in den quantitativen Aufnahmen Veränderung der Myelinisierung. Die späteste erkennbare Myelinisierung war in der Kontrollgruppe im Bereich des temporalen und parietalen Subkortex mit circa fünf Jahren (s. Abb. 5).

Die DTI-Parameter-Karten der Daten, die mit einer EPI- oder einer STEAM-Sequenz aufgenommen wurden, waren in der visuellen Betrachtung weitgehend übereinstimmend, ebenso wie die Aufnahmen mit den Richtungsprotokollen STEAM_P1 und STEAM_P2. 


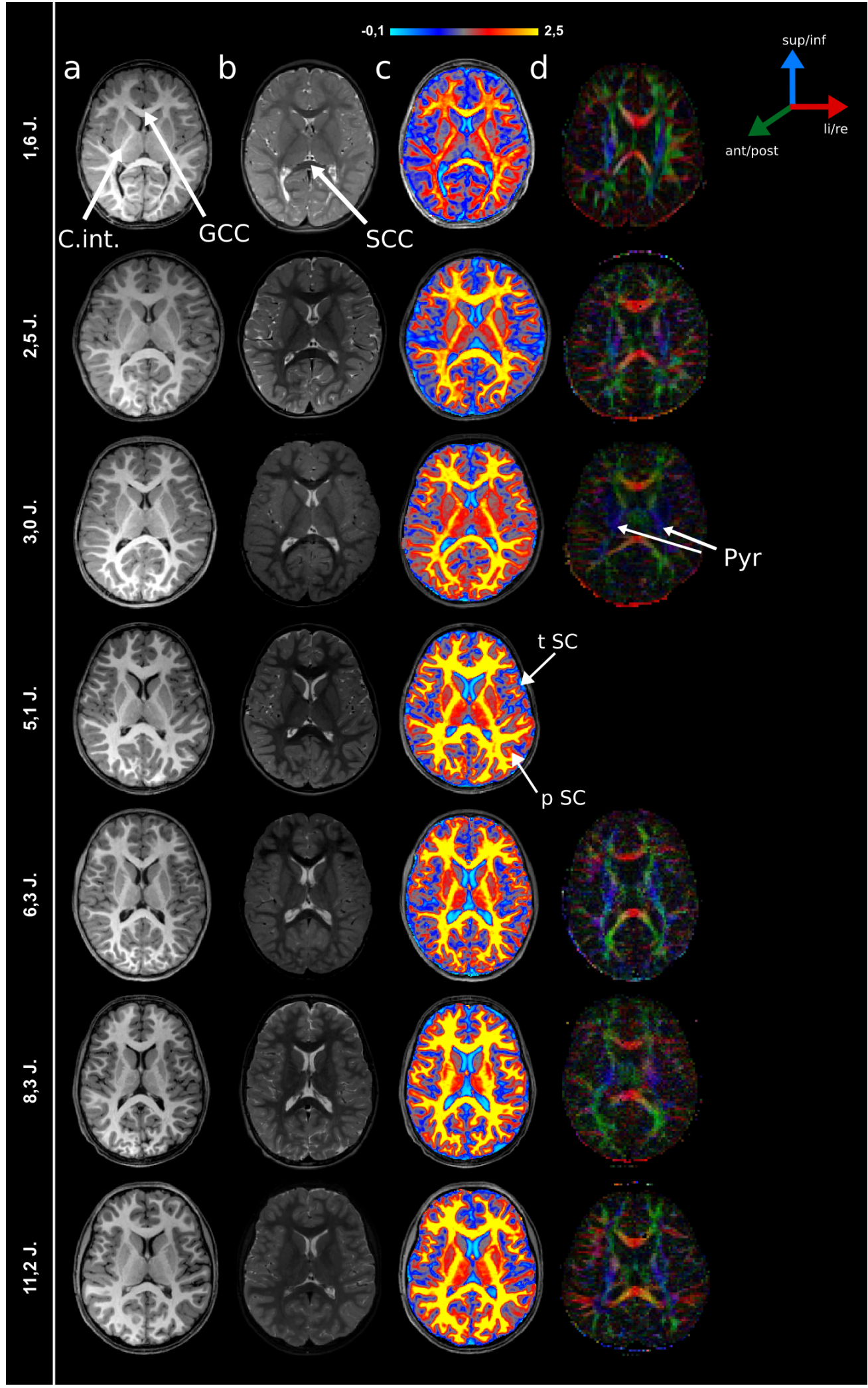

Abb. 5: Verlauf der Myelinisierung in der Kontrollgruppe

a: axiales T1-w Bild; b: axiales T2-w Bild; c: Koregistrierung der farbkodierten MTsat-Karte auf das korrespondierende axiale T1-w-Bild; d: axiale ccFA-Karte. Linke Spalte: Alter der Probanden (in J.). Farbbalken über der Spalte c illutriert Farbkodierung der MTsat-Werte (in p. u.). Gelb: Myelinisierte WM (MTsat $\geq 2,5$ p. u.). Pfeile rechte Spalte: Farbkodierung der ccFA-Karte. Rot: Hauptdiffusionsrichtung links/rechts. Grün: anterior/posterior. Blau: superior/inferior. C.int: Capsula interna. GCC: Genu. SCC: Splenium. t SC: temporaler Subkortex. p SC: parietaler Subkortex. Pyr: Pyramidenbahn. 
3.1.1.2 Quantitative Beschreibung der Diffusionstensor- und MagnetisierungstransferBildgebung

Innerhalb der Kohorte 1 ( $\mathrm{n}=4$, Altersbereich 1,6-5 Jahre) war unabhängig vom verwendeten Aufnahmeprotokoll eine Zunahme der FA sowie eine Abnahme der RD und MD zu beobachten (s. Tabelle A-1). Die AD nahm ebenfalls leicht ab. Im Corpus Callosum wurde vom jüngsten Probanden bis zum ältesten Probanden der Kohorte 1 ein Anstieg der FA von 0,74 a. u. (1,6 Jahre) auf 0,77 a. u. (4,1 Jahre) gemessen. Die AD fiel von 1,86 $10^{3} \mathrm{~mm}^{2} / \mathrm{s}^{-2}$ (1,6 Jahre) auf $1,78 \cdot 10^{3} \mathrm{~mm}^{2} / \mathrm{s}^{-2}$ (4,1 Jahre) ab, genauso wie die RD von $0,42 \cdot 10^{3} \mathrm{~mm}^{2} / \mathrm{s}^{-}$ 2 (1,6 Jahre) auf $0,34 \cdot 10^{3} \mathrm{~mm}^{2} / \mathrm{s}^{-2}$ (4,1 Jahre) und die MD von $0,90 \cdot 10^{3} \mathrm{~mm}^{2} / \mathrm{s}^{-2}(1,6 \mathrm{Jahre})$ auf $0,82 \cdot 10^{3} \mathrm{~mm}^{2} / \mathrm{s}^{-2}$ (4,1 Jahre). Die MTsat stieg im Genu von 2,52 p. u. (1,6 Jahre) auf 3,27 p. u. (4,1 Jahre) an.

Im Vergleich der Kohorten 1 und 2 ( $\mathrm{n}=$ 20, Altersbereich 5-15 Jahre) wurde im Genu, im Splenium, im Corpus Callosum sowie in der Pyramidenbahn eine mit dem Alter zunehmende FA und AD sowie eine abnehmende RD und MD gemessen. Im Genu wurde ein Anstieg der FA bei den Probanden der zwei Protokollreihen STEAM_P2 und EPI von der Kohorte 1 zur Kohorte 2 festgestellt. Die AD fiel im Genu in allen drei Reihen im Verlauf $\mathrm{ab}$, ebenso wie die RD in zwei der drei Kontrollreihen. Die MTsat zeigte die deutlichste Veränderung im Genu mit zunehmendem Alter. Es kam im Schnitt zu einem Anstieg von 2,71 p. u. in der MT-Kohorte 1 ( $\mathrm{n}=4$, Altersbereich 1,6-5 Jahre) auf 3,05 p. u. in der MTKohorte 2 ( $\mathrm{n}=27$, Altersbereich 5-15 Jahre).

Im Splenium zeigte sich in allen drei Protokoll-Gruppen ein FA- und AD-Anstieg sowie ein RD-Abfall von der jüngeren zur älteren Kontrollgruppe. Die MTsat stieg von 2,74 \pm 0,27 p. u. auf 2,89 $\pm 0,18$ p. u. an. Im gesamten Corpus Callosum ließen sich die gleichen Veränderungen der DTI-Parameter, die auch im Splenium im Verlauf von der jüngeren zur älteren Kontrollgruppe gemessen wurden, nachweisen. In der Pyramidenbahn war ein FAAnstieg erkennbar, dazu ein AD- und RD-Abfall.

Sowohl in der WMLF von 2,62 \pm 0,33 p. u. (MT-Kohorte 1) auf 2,82 \pm 0,16 p. u. (MTKohorte 2) als auch in der WMLPO von 2,52 $\pm 0,24$ p. u. auf 2,74 $\pm 0,12$ p. u. stieg die MTsat von der jüngeren zur älteren Kontrollgruppe an.

In der quantitativen Auswertung zeigten sich leichte Unterschiede zwischen den mit EPIund STEAM-Sequenz aufgenommenen DT-Daten sowie zwischen den zwei verschieden Richtungsprotokollen der STEAM-Sequenz (s. Tabelle A-1). Die mit einer EPI-Sequenz aufgenommenen FA-, AD- und MD-Werte waren im Schnitt oberhalb der mit STEAMSequenz aufgenommenen Werte, die RD-Werte etwas unterhalb dieser. 


\subsection{Entzündliche Demyelinisierung - Kindliche-zerebrale-X- chromosomale-Adrenoleukodystrophie}

\subsubsection{Verlaufsbeschreibung der Myelinparameter bei Patient 1}

\subsubsection{Qualitative Beurteilung der MRT-Aufnahmen}

Mit 4,4 Jahren war bei Patient 1 in der MTsat-Karte im Kolorierungsbereich -0,1 bis 2,5 p. u. ein diffuses, unscharf begrenztes Areal mit verminderten MTsat-Werten (rot in der Farbskala) zu erkennen (s. Abb. 6). In den Aufnahmen der anderen MR-Modalitäten zeigten sich in diesem Alter keine Veränderungen.

Im Alter von fünf Jahren, sechs Monate vor der radiologischen Diagnosestellung der ccALD, fiel retrospektiv bei Patient 1 im Splenium in der T2-w Aufnahme eine schwache Hyperintensität mit einem Durchmesser von eineinhalb Millimeter auf (s. Abb. 6). Die T1-w Aufnahme, die ccFA-Karte und die MTsat-Karte (Farbskala $-0,1$ bis 2,5 p. u.) waren zu diesem Zeitpunkt unauffällig hinsichtlich einer Läsion. Nach Anpassung des Kolorierungsbereiches der MTsat-Karte auf Werte von -0,1 bis 2,7 p. u. traten mit fünf Jahren im Areal der Läsion rötliche Verfärbungen mit verminderten MTsat-Werten hervor (s. Abb. 7). Verminderte MTsat-Werte wurden bei der Auswahl dieses Farbbereiches auch in Arealen des Corpus Callosum gemessen, in denen in folgenden Untersuchungen keine Läsionen bestätigt worden sind.

Die Diagnose ccALD wurde bei Patient 1 radiologisch nach Beschreibung der Läsion im Splenium mit 5,6 Jahren gestellt. In diesem Alter zeigte sich eine scharfbegrenzte, runde T1hypointense, T2-hyperintense, KM-aufnehmende Läsion mit einem Durchmesser von fünf Millimeter (s. Abb. 6). Am deutlichsten imponierte die Läsion in der farbkodierten MTsatKarte und der T2-w Aufnahme. In der ccFA-Karte stellten sich zwei Voxel im Areal der Läsion im Vergleich zum übrigen Splenium dunkler dar. Dieses zeigte eine niedrigere FA an (s. Abb. 6, ccFA-Karte, weißer Pfeil).

Mit 7,3 Jahren, 19 Monate nach der HSZT, wurde eine Kontrolluntersuchung bei Patient 1 durchgeführt. Diese stellte das demyelinisierte Areal im Splenium als T1-hypointens, T2hyperintens, und diffusionserleichtert ohne KM-Aufnahme dar (s. Abb. 6). Im Vergleich zur Voruntersuchung hatte die Läsion etwas an Größe zugenommen (Durchmesser: sieben Millimeter). 


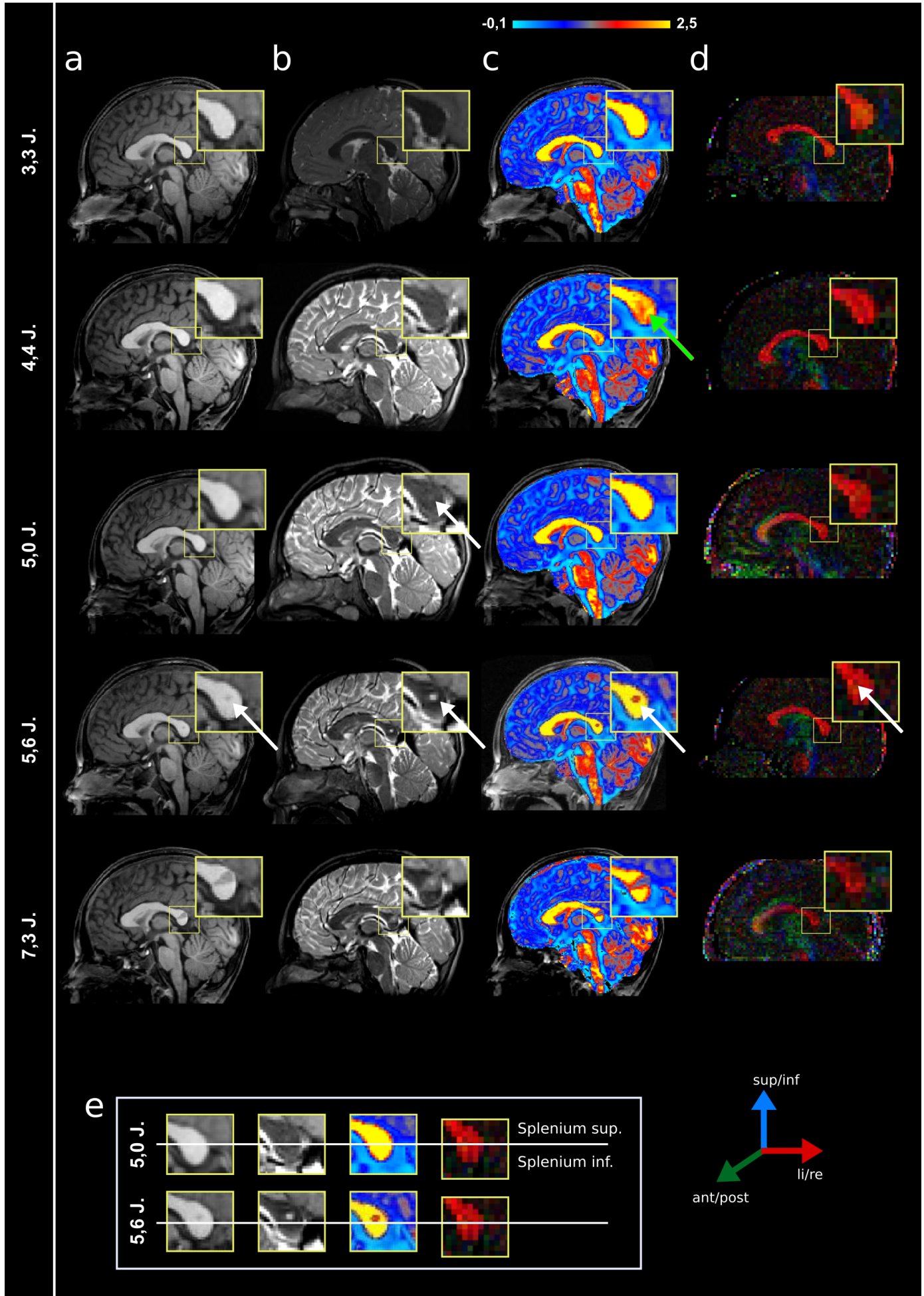

Abb. 6: MRT-Aufnahmen des ccALD-Patienten 1

a: axiales T1-w Bild; b: axiales T2-w Bild; c: Koregistrierung der farbkodierten MTsat-Karte auf das korrespondierende axiale T1-w-Bild; d: axiale ccFA-Karte. e: Vergrößerung der SpleniumAuschnitte der Messungen mit fünf und fünfeinhalb Jahren. Spleniumbereich oberhalb der Linie: Splenium superior (Splenium sup.). Spleniumbereich unterhalb der Linie: Splenium inferior (Splenium inf.). Weißer Pfeil: Läsion im Splenium; Grüner Pfeil: Areal diffus erniedrigter MTsatWerte 


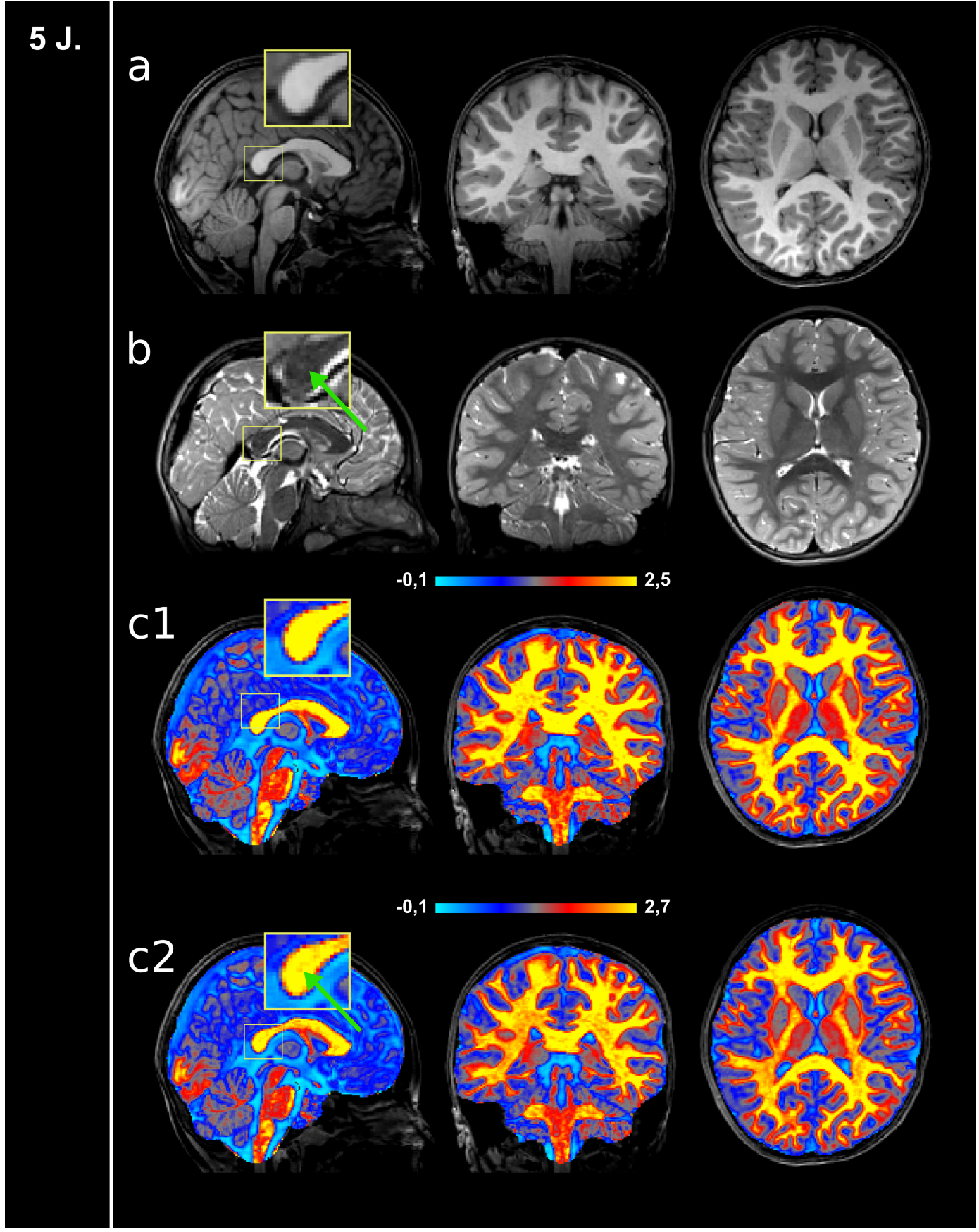

Abb. 7: Darstellung der Läsionen des Patienten 1 mit fünf Jahren

a: axiales T1-w Bild; b: axiales T2-w Bild; c1: Koregistrierung der farbkodierten MTsat-Karte auf das korrespondierende axiale T1-w-Bild (Farbskala -0,1 p. u. bis 2,5 p.u); c2: Koregistrierung der farbkodierten MTsat-Karte auf das korrespondierende axiale T1-w-Bild (Farbskala -0,1 p. u. bis 2,7 p.u). Grüne Pfeile: Hyperintensität in T2-w Aufnahme (b) und verminderte MTsat-Werte (rot in der Farbskala) in MTsat-Karte (c2) im Splenium als Hinweis auf Läsion

\subsubsection{Verlaufsbeschreibung mittels Diffusionstensor-Bildgebung}

In den Verlaufsmessungen der quantitativen DTI zeigte sich im Splenium (GesamtSplenium) bei Patient 1 ein Abfall der FA von 0,84 $\pm 0,02$ a. u. (2,5 Jahre) auf 0,73 $\pm 0,02$ a. u. (5,6 Jahre) (s. Abb. 8, s. Tabelle A-2). In der Messung mit 7,3 Jahren (19 Monate postHSZT) war die FA auf 0,55 \pm 0,01 a. u. gefallen. Im Splenium superior, in dem die Läsion sich manifestierte, fiel die FA von 0,82 $\pm 0,02$ a. u. (2,5 Jahre) auf 0,73 $\pm 0,02$ a. u. (5,6 Jahre) 
und auf 0,44 \pm 0,03 a. u. (7,3 Jahre) (s. Abb. 8, s. Tabelle A-2). In den Messungen vor der HSZT waren die FA-Werte im Splenium innerhalb des Normbereiches. Die Werte der Messungen mit fünf und fünfeinhalb Jahren waren am unteren Rand dieses. Nach der HSZT wurden FA-Werte in allen drei Splenium-ROI unterhalb des Normbereiches gemessen. Die AD lag im Splenium, im Splenium superior und im Splenium inferior ab dem Alter von vier Jahren konstant im unteren Normbereich (s. Abb. 8). Einzelne Werte mit fünf, 5,6 und 7,3 Jahren lagen leicht unterhalb des Normbereiches. Die RD stieg im Splenium bei Patient 1 von $0,21 \cdot 10^{-3} \mathrm{~mm}^{2} \cdot \mathrm{s}^{-1}(2,5 \mathrm{Jahre})$ auf $0,30 \cdot 10^{-3} \mathrm{~mm}^{2} \cdot \mathrm{s}^{-1}$ (5,6 Jahre) und im Splenium superior von $0,24 \cdot 10^{-3} \mathrm{~mm}^{2} \cdot \mathrm{s}^{-1}(2,5 \mathrm{Jahre})$ auf $0,30 \cdot 10^{-3} \mathrm{~mm}^{2} \cdot \mathrm{s}^{-1}(5,6 \mathrm{Jahre})$ an. Die MD fiel zwischen zweieinhalb und fünf Jahren ab, stieg in der Folge jedoch soweit an, dass sie mit 7,3 Jahren oberhalb des Kontrollbereiches lag.

Im Areal der Läsion lagen die FA-, AD- und RD-Werte zum Zeitpunkt der radiologischen Diagnosestellung mit 5,6 Jahren außerhalb des Normbereiches (s. Abb. 8). Die FA- und ADWerte waren unterhalb dieses, die RD-Werte oberhalb (FA: 0,53 a. u.; AD: $1,14 \cdot 10^{-3} \mathrm{~mm}^{2} \cdot \mathrm{s}^{-1}$; RD: $0,45 \cdot 10^{-3} \mathrm{~mm}^{2} \cdot \mathrm{s}^{-1}$ ) (s. Tabelle A-2). Sechs Monate zuvor wurden in der gleichen Lokalisation die FA, AD- und RD-Werte außerhalb des Normbereiches gemessen. Die MDWerte lagen mit fünf Jahren im mittleren Normalbereich (s. Tabelle A-2). Nach der HSZT fiel die FA in der Läsion auf 0,36 a. u. und die AD auf $1,01 \cdot 10^{-3} \mathrm{~mm}^{2} \cdot \mathrm{s}^{-1} \mathrm{ab}$. Die RD stieg an auf $0,57 \cdot 10^{-3} \mathrm{~mm}^{2} \cdot \mathrm{s}^{-1}$ und die MD auf $0,62 \cdot 10^{-3} \mathrm{~mm}^{2} \cdot \mathrm{s}^{-1}$.

\subsubsection{Verlaufsbeschreibung mittels Magnetisierungstransfer-Bildgebung}

Die MTsat stieg ausgehend von Werten im mittleren Normbereich mit zweieinhalb Jahren bis zur Messung mit dreieinhalb Jahren in den drei Regionen Splenium, Splenium superior an (s. Abb. 8, s. Tabelle A-2). Mit 4,4 Jahren lag die MTsat mit Werten zwischen 2,23 p. u. und 2,45 p. u. in allen drei Regionen unterhalb des Normbereiches. In der folgenden Messung mit fünf Jahren war die MTsat wieder innerhalb des Normbereiches (Werte zwischen 2,72 p. u. bis 2,73 p. u.). Anschließend fiel die MTsat ab. Mit 5,6 Jahren, dem Zeitpunkt der radiologischen Feststellung der entzündlichen Demyelinisierung, lag die MTsat im Splenium bei 2,64 \pm 0,21 p. u. und im Splenium superior bei 2,55 \pm 0,44 p. u. Letzterer Wert befand sich unterhalb der Werte der Kontrollgruppe. In der Messung nach der HSZT wurden MTsat-Werte von 2,10 \pm 0,60 p. u. (Gesamt-Splenium) und von 1,22 \pm 0,19 p. u. (Splenium superior) gemessen. Diese lagen unterhalb des Normbereiches. Die MTsat-Werte im Splenium inferior waren durchweg innerhalb des Normalbereiches.

In den ROI-basierten Messungen wurden im Bereich der Läsionen zum Zeitpunkt der Diagnosestellung eine deutlich verminderte MTsat gemessen. Sechs Monate zuvor war die MTsat an der unteren Grenze des Normbereiches. Mit 4,4 Jahren lag die MTsat ebenfalls unterhalb des Normbereiches. Insgesamt zeigte sich ein deutlicher Abfall der MTsat im Verlauf. 

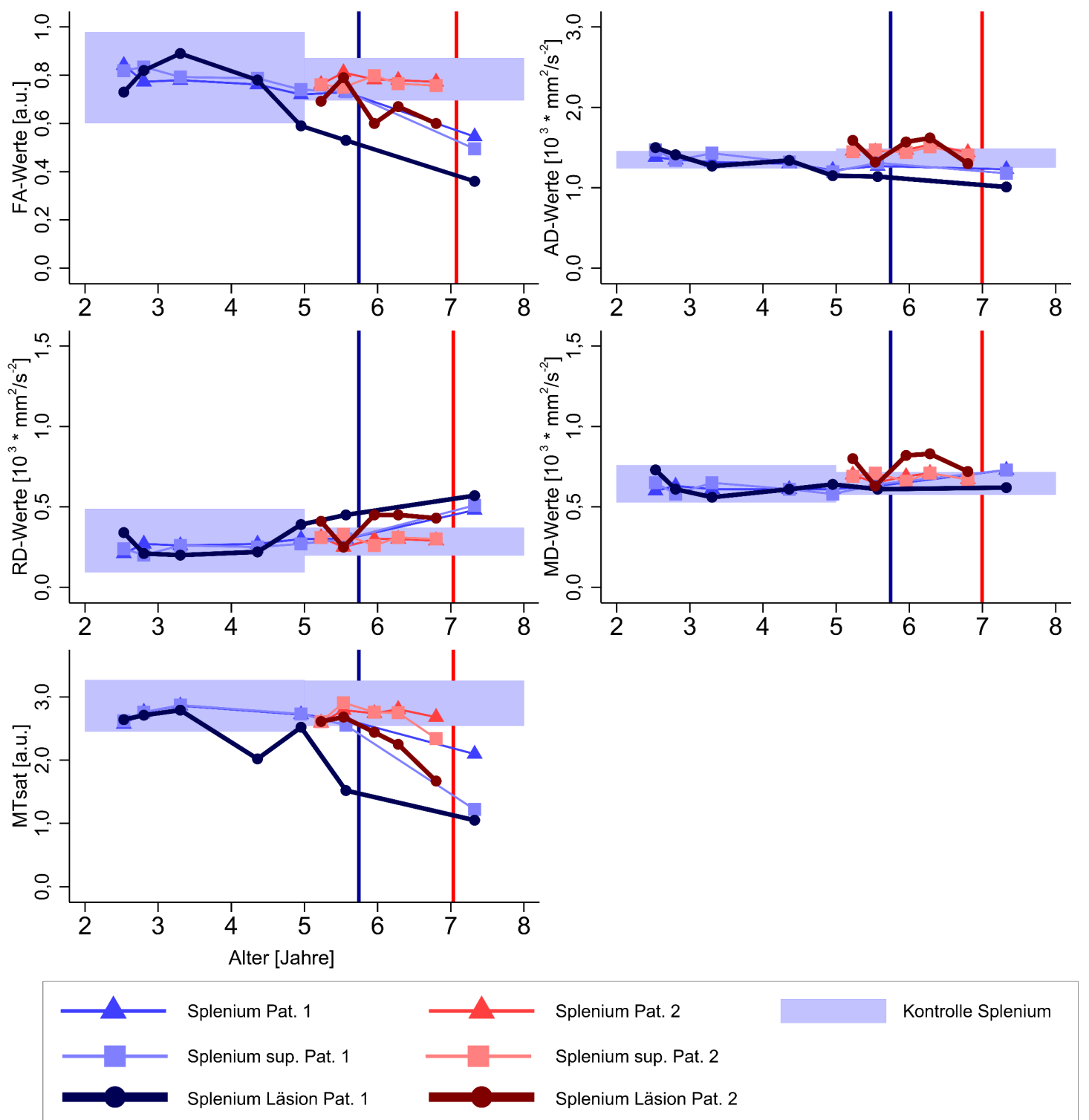

Abb. 8: Verlauf der myelinsensitiven Parameter der ccALD-Patienten 1-2

Blaue Graphen: DTI/MTsat-Werte Patient 1 (Pat. 1). Rote Graphen: DTI/MTsat-Werte Patient 2. (Gesamt-)Splenium (Icon: Dreieck), Splenium superior (Icon: Quadrat), Splenium Läsion (Icon: Kreis). Hellblauer Bereich: Normbereich. Vertikaler Strich blau: Zeitpunkt HSZT Patient 1. Vertikaler Strich rot: Patient 2.

\subsubsection{Verlaufsbeschreibung der Myelinparameter bei Patient 2}

\subsubsection{Qualitative Beurteilung der MRT-Aufnahmen}

Die konventionellen MRT-Aufnahmen, die MTsat- und die ccFA-Karten zeigten bei Patient 2 bis zum Alter von fünfeinhalb Jahren einen Normalbefund (s. Abb. 9). Retrospektiv fiel in der T2-w Aufnahme mit sechs Jahren, zehn Monate vor der erstmaligen radiologischen Beschreibung, eine hyperintense Läsion im Splenium mit einem Durchmesser von eineinhalb Millimeter auf (s. Abb. 9, weißer Pfeil). In der MTsat- und der ccFA-Karte war dieses Areal mit reduzierten MTsat (rot in der Farbskala) bzw. FA-Werte (niedrigere 
Farbintensität als Umgebung) ebenfalls auffällig (s. Abb. 9). In der Folge-Untersuchung mit 6,3 Jahren zeigte sich die Läsion in der T2-w Aufnahme, der MTsat- und der ccFA-Karte leicht vergrößert. Die Diagnose ccALD wurde bei Patient 2 mit 6,8 Jahren nach der radiologischen Beschreibung einer T1-hypointensen und T2-hyperintensen runden Läsion mit einem Durchmesser von drei Millimeter gestellt. In der MTsat-Karte imponierte die Läsion zu diesem Zeitpunkt als strichförmige Läsion mit einer Breite von drei Millimetern und einer Länge von sechs Millimetern. In der ccFA-Karte war ein langgezogenes Areal mit verminderter Farbintensität (niedrigere FA-Werte) zu erkennen (s. Abb. 9). Die post-HSZT MRT-Untersuchungen erfolgten mit 9,9 und 10,8 Jahren. Die Läsion stellte sich in den Untersuchungen als scharfbegrenztes feinlineares Residuum ohne Diffusionsnachweis, ohne KM-Aufnahme und ohne weitere Größenzunahme dar (s. Abb. 9). qMRT-Messungen wurden mit 9,9 und 10,8 Jahren nicht durchgeführt.

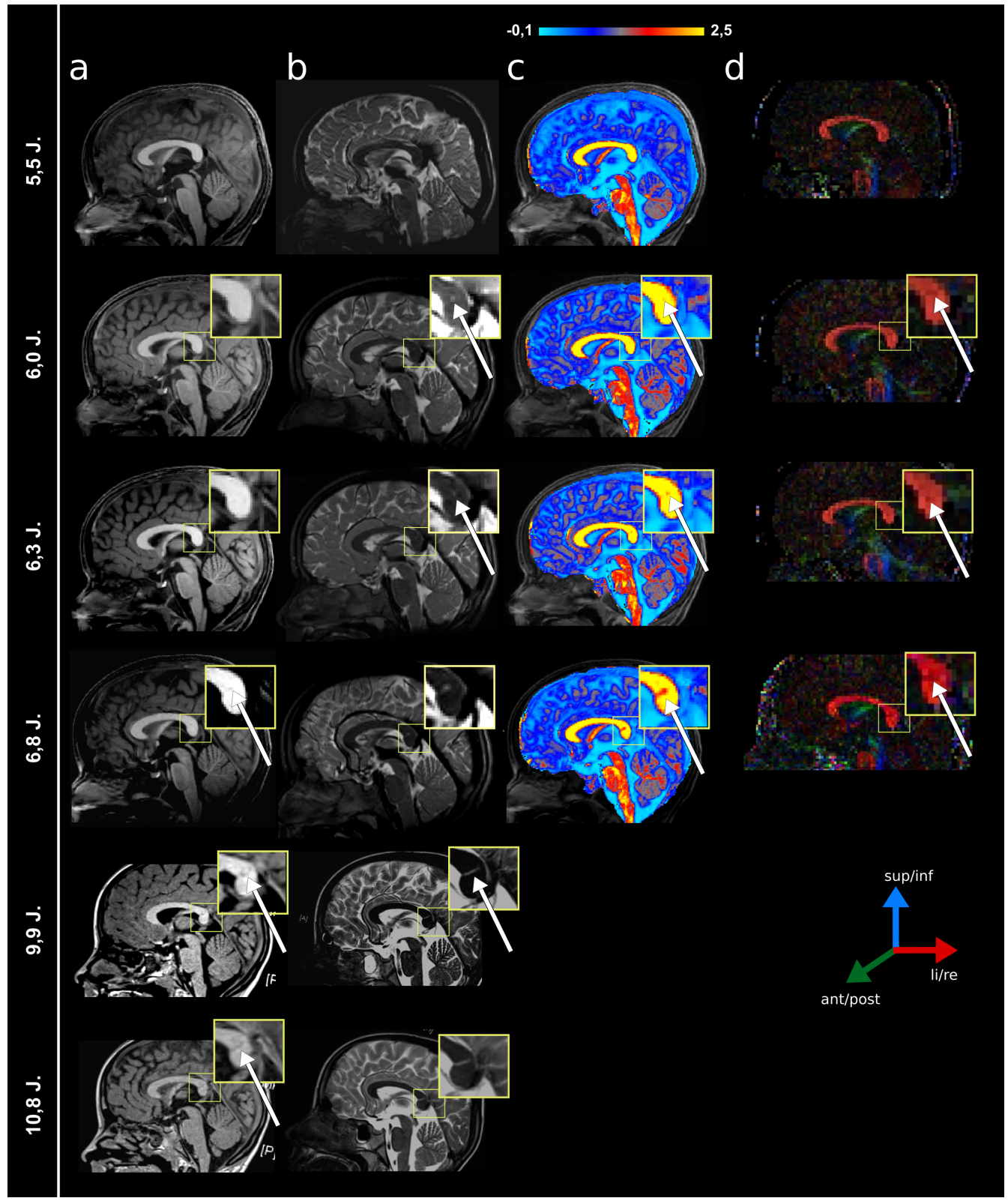

Abb. 9: MRT-Aufnahmen des ccALD-Patienten 2 
a: axiales T1-w Bild; b: axiales T2-w Bild; c: Koregistrierung der farbkodierten MTsat-Karte auf das korrespondierende axiale T1-w-Bild; d: axiale ccFA-Karte. Vergrößerungen der T1-w und T2-w Bilder mit geänderten Kontrast- und Helligkeitseinstellung zur besseren Visualisierung. Weiße Pfeile: Läsion im Splenium des Patienten

\subsubsection{Verlaufsbeschreibung mittels Diffusionstensor-Bildgebung}

Die Diffusionsparameter FA und RD lagen bei Patient 2 in den ROI Gesamt-Splenium und Splenium superior konstant im Normbereich (s. Abb. 8, s. Tabelle A-3). An der oberen Grenze des Kontrollbereichen waren die AD- und MD-Werte zum Zeitpunkt vor der Diagnosestellung im Gesamt-Splenium und Splenium superior. Im Splenium inferior lagen die AD und MD mit 5,5 Jahre, 6 Jahre und 6,3 Jahre sogar über dem Normbereich. Die AD fiel in allen Splenium-Regionen in der Messung zum Zeitpunkt der radiologischen Diagnosestellung wieder leicht ab, ebenso wie die MD.

In der Läsion lagen die FA-Werte mit 6,8 Jahren unterhalb des Normbereiches und die RDund MD-Werte oberhalb (FA: 0,60 a. u.; AD: $1,30 \cdot 10^{-3} \mathrm{~mm}^{2} \cdot \mathrm{s}^{-1}$; RD: $0,43 \cdot 10^{-3} \mathrm{~mm}^{2} \cdot \mathrm{s}^{-1}$; MD: $0,72 \cdot 10^{-3} \mathrm{~mm}^{2} \cdot \mathrm{s}^{-1}$ ) (s. Abb. 8 , s. Tabelle A-3). In den Messungen sechs und zehn Monate zuvor wurden in der gleichen Lokalisation bereits FA-Werte unterhalb und RD- und MDWerte oberhalb des Normbereiches gemessen (s. Abb. 8).

\subsubsection{Verlaufsbeschreibung mittels Magnetisierungstransfer-Bildgebung}

Nachdem die MTsat im (Gesamt-)Splenium, Splenium superior und inferior zunächst anstieg, fiel sie ab dem Alter von 5,5 Jahren leicht ab. Die Werte im Splenium und Splenium inferior lagen in der Messung zum Zeitpunkt der Diagnosestellung am unteren Rand des Kontrollbereiches. Im Splenium superior wurden mit 2,34 \pm 0,29 p. u. (6,8 Jahre) Werte unterhalb des Normbereiches gemessen.

Mit sechs Jahren, zehn Monate vor der Diagnosestellung, wurden in der Läsion MTsat-Werte von 2,44 \pm 0,08 p. u. gemessen, mit 6,3 Jahren 2,25 $\pm 0,09$ p. u. und mit 6,8 Jahren 1,67 \pm 0,09 p. u. (s. Abb. 8). Diese lagen unterhalb des Normbereiches. Bei vollständiger Myelinisierung waren MTsat-Werte im Splenium > 2,5 zu erwarten. 


\subsection{Hypomyelinisierung - Pelizaeus-Merzbacher-Syndrom}

Im Folgenden sind die DT- und MT-Daten der fünf Patienten mit PMS (Patient 3-7) im Alter von eineinhalb bis elf Jahren aufgeführt. Die Patienten sind nach Ausprägung der klinischen Symptomatik nummeriert. Patient 3 wies die mildeste Klinik auf, Patient 7 die ausgeprägteste. Darüber hinaus wurden die Patienten in die Gruppen PMS0, PMS1-2, PMS3 und PMS4 eingeteilt (vgl. Kapitel 2.1.3.1).

\subsubsection{Verlaufsbeschreibung der Myelinparameter der Patienten 3 bis 7}

\subsubsection{Qualitative Beurteilung der MRT-Aufnahmen}

Bei allen fünf untersuchten Patienten imponierte eine globale Hypomyelinisierung im MRT. Sie zeigte sich durch ein T1-hypointenses und T2-hyperintenses Signal in der WM (s. Abb. 10-14). In den MTsat Karten dominierten niedrige Werte in der WM (blau bis grau in der Farbskala). In den ccFA-Karten wurden bei allen Patienten niedrigere FA-Werte als bei den Kontrollen angezeigt. In einzelnen Arealen waren bei den Patienten 3 bis 6 hyperintense Signale in den T1-w Aufnahmen und hypointense Signale in den T2-w Aufnahmen zu sehen (s. Abb. 10-14). Dieses sind Hinweise auf eine Myelinisierung (s. Abb. 10, 11). In der MTBildgebung imponierten in myelinisierte Arealen höhere MTsat-Werte (rot-orangen in der Farbskala). Bei Patient 3 waren mit 7,9 Jahren Fasern paraventrikulär, im Bereich der Pyramidenbahn und okzipito-subkortikal, im Bereich der Sehstrahlung, myelinisiert (s. Abb. 10). Global zeigte sich ein ausgeprägtes Myelindefizit (s. Abb. 10).

Patient 4 zeigte in der Untersuchung mit 1,6 Jahren eine deutliches Myelindefizit (s. Abb. 11). Fünf Jahre später waren jedoch fleckenförmig Areale im Corpus Callosum, im Bereich der Pyramidenbahn und im Subkortex myelinisiert. Die vorangeschrittene Myelinisierung zeigte sich insbesondere in den T1-w, den T2-w Aufnahmen und der MTsat-Karte.

Die konventionellen Aufnahmen von Patient 5 sowie die MTsat-Karten zeigten mit 3,2 und mit 4,2 Jahren ein Myelindefizit (s. Abb. 12). In der T1-w Aufnahme mit 3,2 Jahren war ein diskretes hyperintenses Signal beidseits im Bereich der Pyramidenbahn zu sehen (s. Abb. 12). Mit 4,1 Jahren war dieses ausgeprägter. Die MTsat-Werte lagen im Bereich der Pyramidenbahn mit 4,1 Jahren zwischen 1,8 und 2,3 p. u. (rot in der Farbskala) (s. Abb. 12).

In den Aufnahmen der Patienten 6 (s. Abb. 13) und 7 (s. Abb. 14) imponierte das Marklager global T1-hypointens und T2-hyperintens. Die MTsat-Werte waren auf $<1,5$ p.u erniedrigt (blau-grau in der Farbskala). Mit zunehmendem Alter wurden ansteigende Werte zwischen 1,8 und 2,3 p. u. in der Pyramidenbahn gemessen (rot in der Farbskala) (s. Abb. 13). Weitere Befundänderungen zeigten sich bei Patient 6 nicht.

Patient 7 wies neben der Hypomyelinisierung, eine globale Hirnvolumenminderung mit Erweiterung der inneren und äußeren Liquorräume auf (s. Abb. 14). Darüber hinaus zeigte sich ein sehr ausgeprägtes Myelindefizit. 


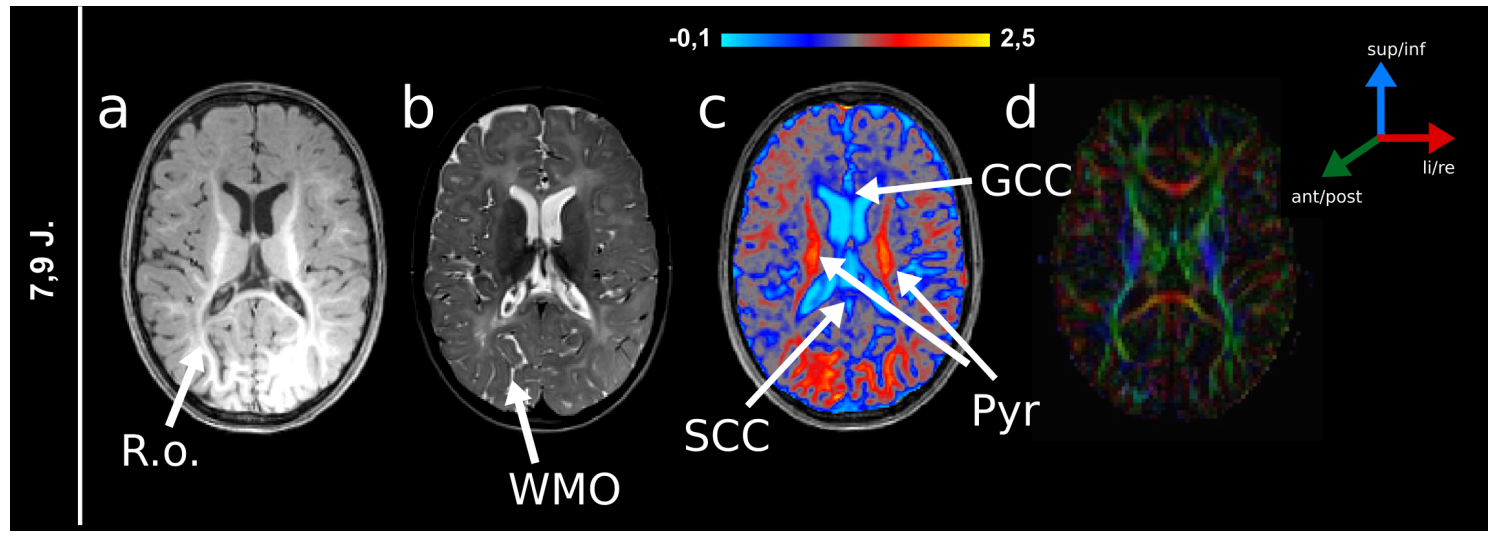

Abb. 10: MRT-Aufnahmen des PMS-Patienten 3

a: axiales T1-w Bild; b: axiales T2-w Bild; c: Koregistrierung der farbkodierten MTsat-Karte auf das korrespondierende axiale T1-w-Bild; d: axiale ccFA-Karte. Weiße Pfeile: Myelinsierung in okzipitaler WM (WMO), Sehstrahlung (R.o.), Pyramidenbahn (Pyr). In Genu (GCC) und Splenium (SCC) deutliches Myelindefizit

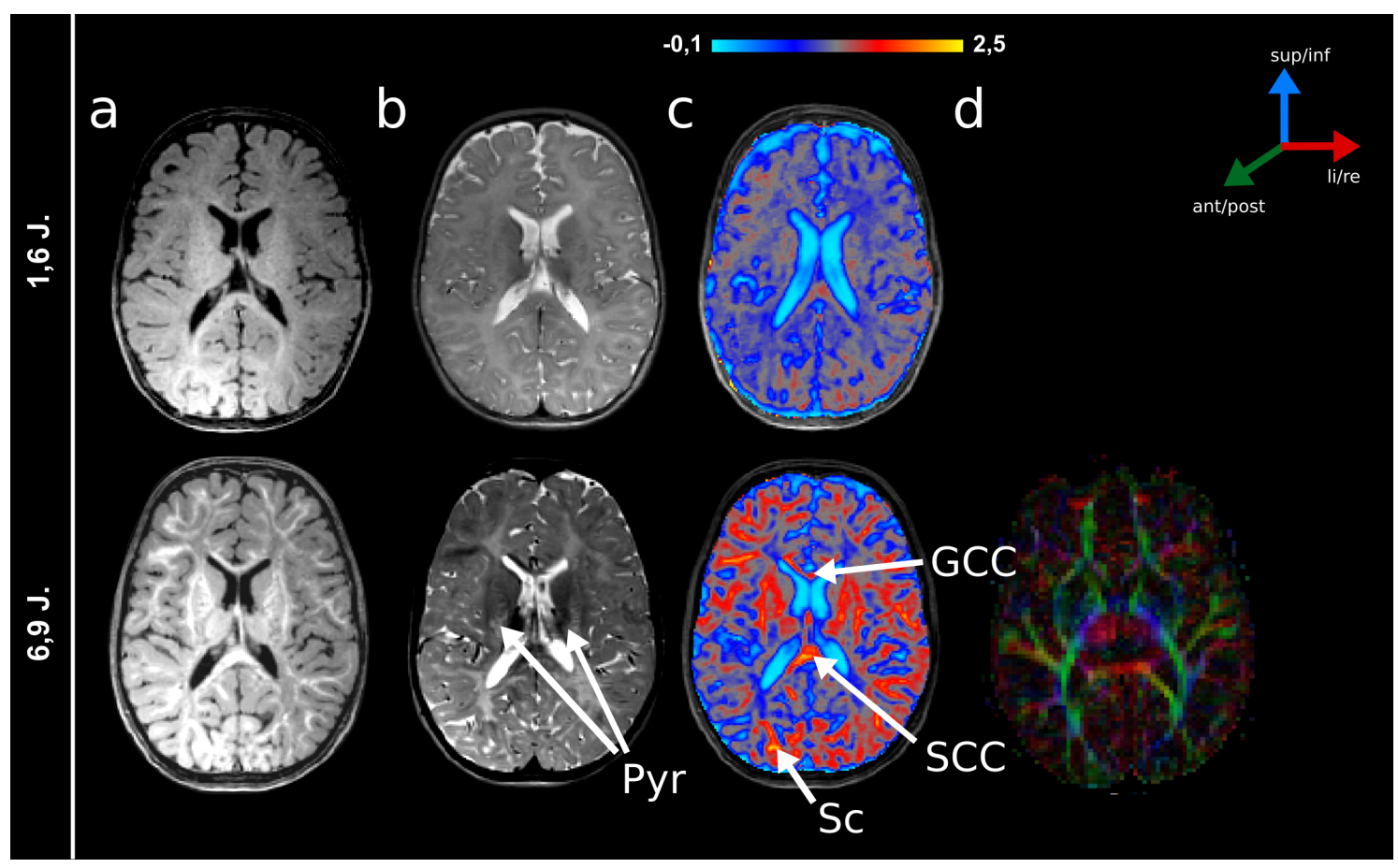

Abb. 11: MRT-Aufnahmen des PMS-Patienten 4

a: axiales T1-w Bild; b: axiales T2-w Bild; c: Koregistrierung der farbkodierten MTsat-Karte auf das korrespondierende axiale T1-w-Bild; d: axiale ccFA-Karte. Weiße Pfeile: Myelinisierung in subkortikalen Abschnitten (Sc), im Genu (GCC), im Splenium (SCC) und der Pyramidenbahn (Pyr). Keine DTI- Aufnahmen mit 1,6 J. 


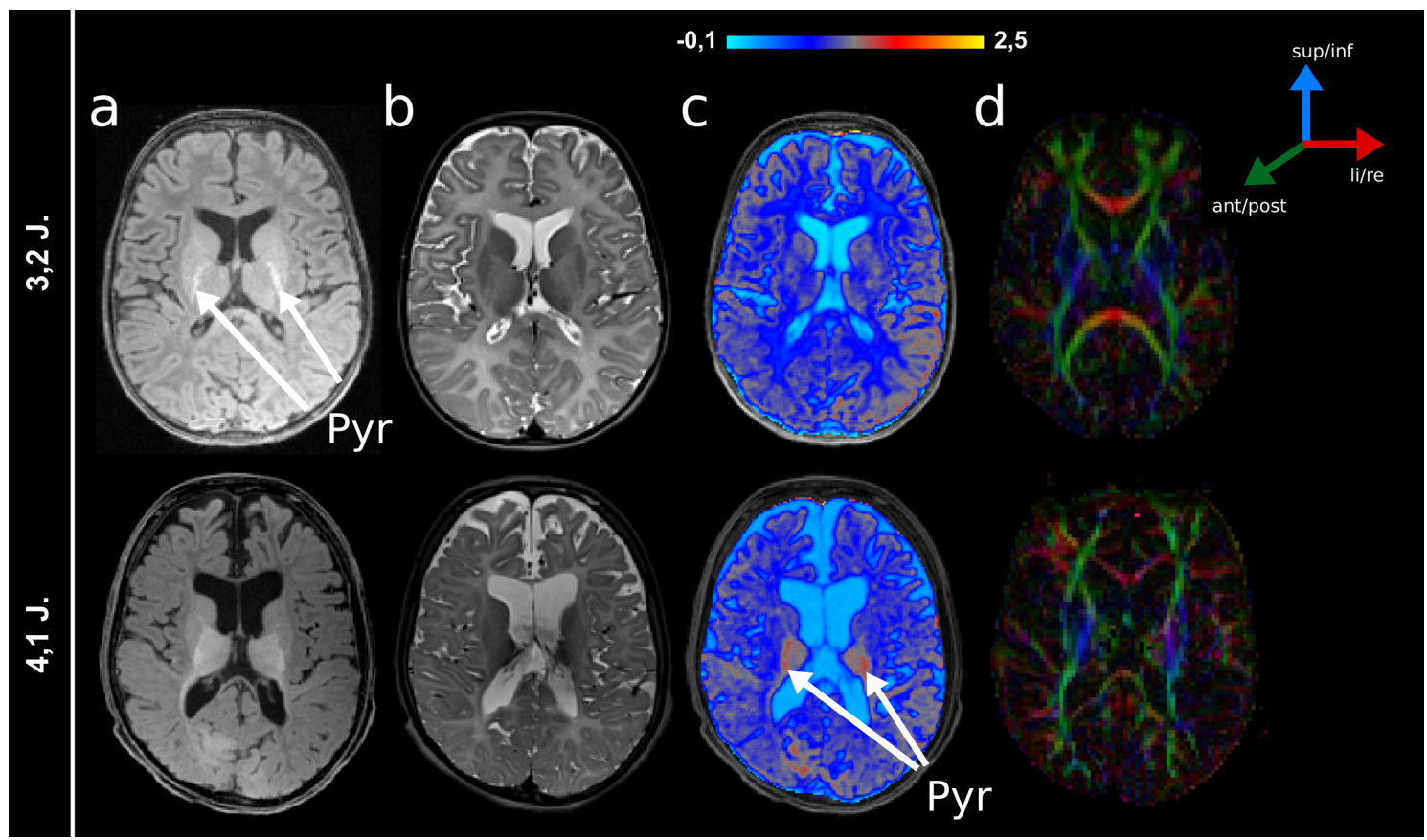

Abb. 12: MRT-Aufnahmen des PMS-Patienten 5

a: axiales T1-w Bild; b: axiales T2-w Bild; c: Koregistrierung der farbkodierten MTsat-Karte auf das korrespondierende axiale T1-w-Bild; d: axiale ccFA-Karte. Weiße Pfeile: Myelinisierung im Bereich der Pyrmidenbahn (Pyr). 


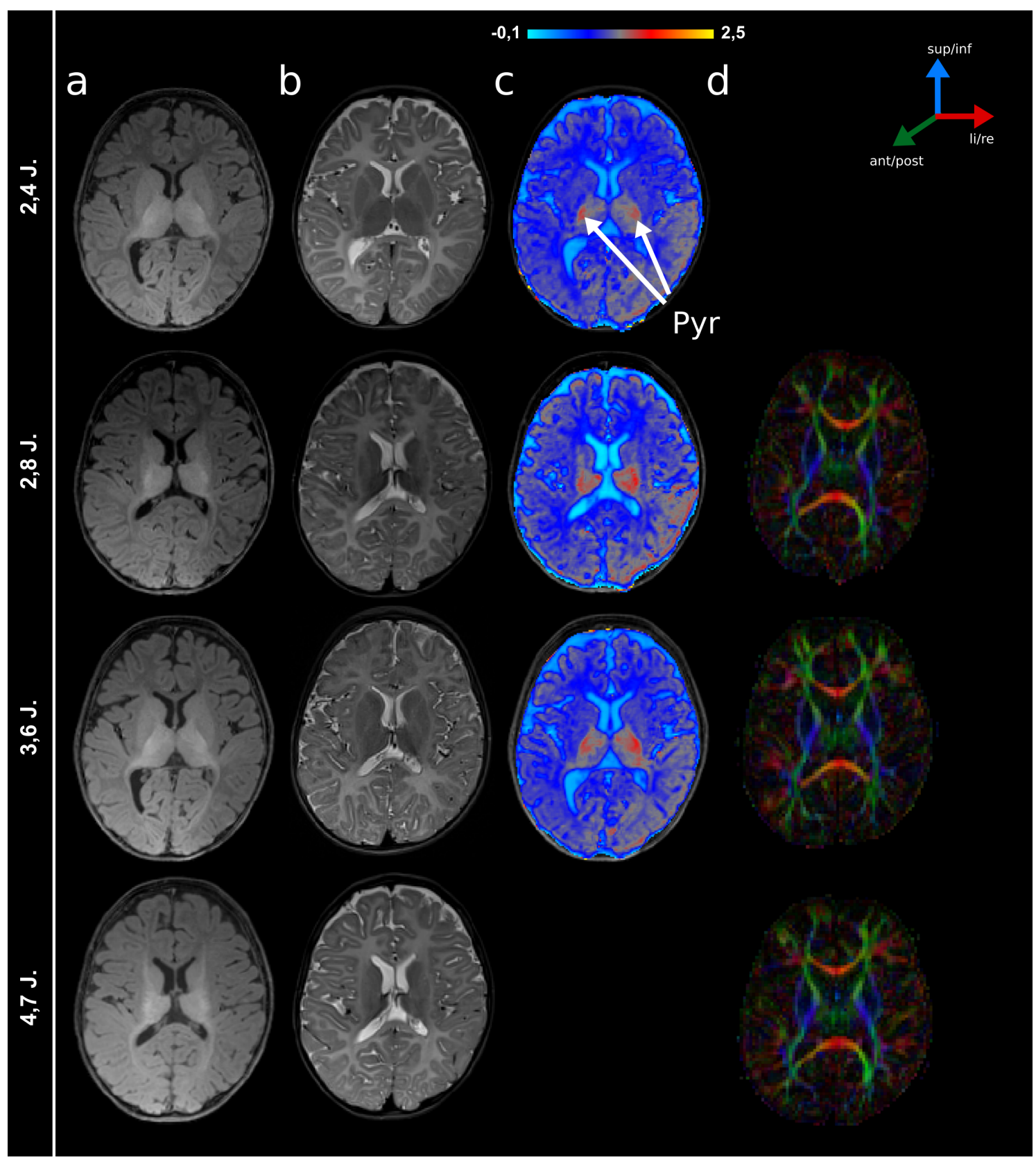

Abb. 13: MRT-Aufnahmen des PMS-Patienten 6

a: axiales T1-w Bild; b: axiales T2-w Bild; c: Koregistrierung der farbkodierten MTsat-Karte auf das korrespondierende axiale T1-w-Bild; d: axiale ccFA-Karte. Weißer Pfeil: Myelinisierung im Bereich der Pyramidenbahn (Pyr). Keine DTI-Daten mit 2,4 J., keine MT- Daten mit 4,7 J. 


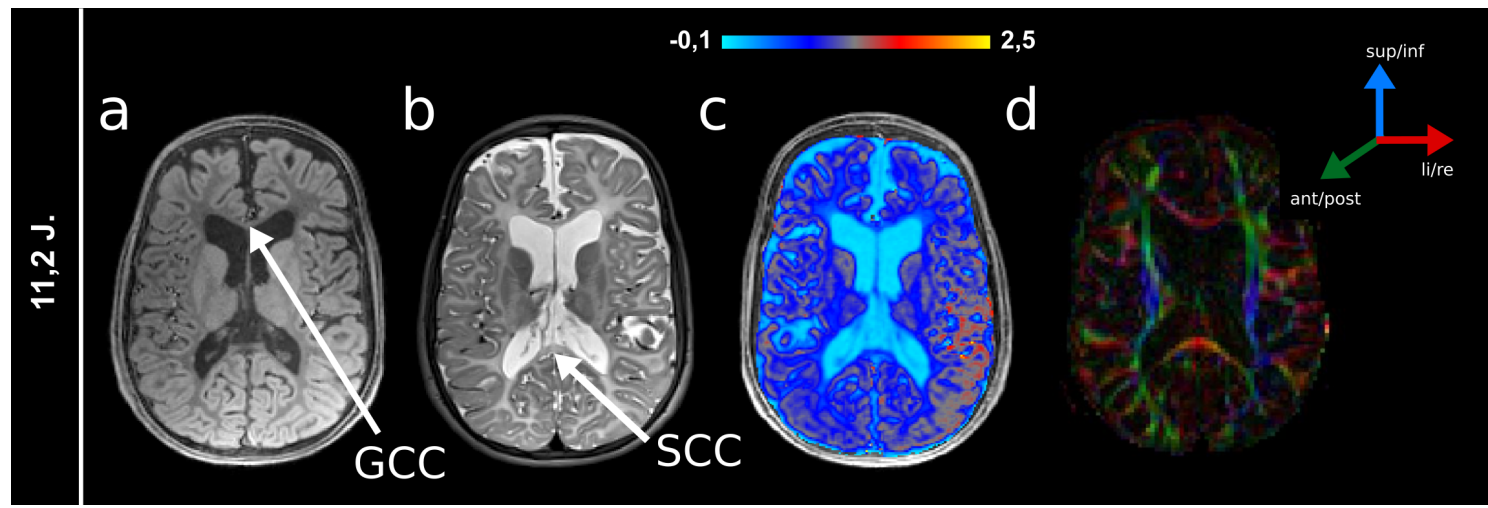

Abb. 14: MRT-Aufnahmen des PMS-Patienten 7

a: axiales T1-w Bild; b: axiales T2-w Bild; c: Koregistrierung der farbkodierten MTsat-Karte auf das korrespondierende axiale T1-w-Bild; d: axiale ccFA-Karte. Anatomische MR-Bildern zeigen die Hirnatrophie. Weiße Pfeile: Genu (GCC), Splenium (SCC)

\subsubsection{Verlaufsbeschreibung mittels Diffusionstensor-Bildgebung}

\subsection{Fraktionale Anisotropie}

Die FA-Werte von Patient 3, dem Patienten mit der mildesten klinischen Ausprägung waren in Genu, Splenium und Corpus Callosum (ROI Corpus Callosum, s. Kapitel 2.2.5) deutlich unterhalb des Kontrollbereiches. Die FA-Werte im Genu und im Splenium waren oberhalb der durchschnittlichen Werte im Corpus Callosum. In der Pyramidenbahn lag die FA leicht unterhalb des Normbereiches (s. Abb. 15, s. Tabelle A-4). Obwohl die klinische Symptomatik bei Patient 3 milder war als bei den Patienten 4, 5 und 6, war die FA in allen vier gemessenen Regionen niedriger und damit stärker vom Normbereich abweichend (s. Abb. 15).

Die FA-Werte von Patient 4 lagen im Splenium und in der Pyramidenbahn im unteren Normbereich, im Genu und im Corpus Callosum leicht darunter. Bei den jüngeren Patienten 5 und 6 waren die FA-Werte in den vier Regionen etwas niedriger als bei Patient 4. Darüber hinaus zeigte sich bei beiden Patienten im Genu und Splenium ein Abfall der FA im Verlauf.

Die FA-Werte von Patient 7 waren in den vier Regionen unterhalb des Normbereiches und niedriger als bei den Patienten 4, 5 und 6. Im Splenium und im Corpus Callosum war der FAWert jedoch höher als bei dem klinisch milder betroffenen Patienten 3 .

In Abb. 16 ist der Zusammenhang der klinischen Symptomatik und der quantitativen Parameter zusammenfassend illustriert (s. Abb. 16). Die FA-Werte in der Kontrollgruppe 5 bis 15 Jahre waren leicht oberhalb der Werte der jüngeren Kontrollgruppe. Mit zunehmender klinischer Symptomatik der Patienten mit PMS wurde eine abnehmende FA im Genu, Splenium, im Corpus Callosum und in der Pyramidenbahn gemessen. Eine Ausnahme stellte die Gruppe PMS0 mit Patient 3 dar. Die FA war bei dieser in Splenium und im gesamten Corpus Callosum sogar unterhalb von Patient 7. 

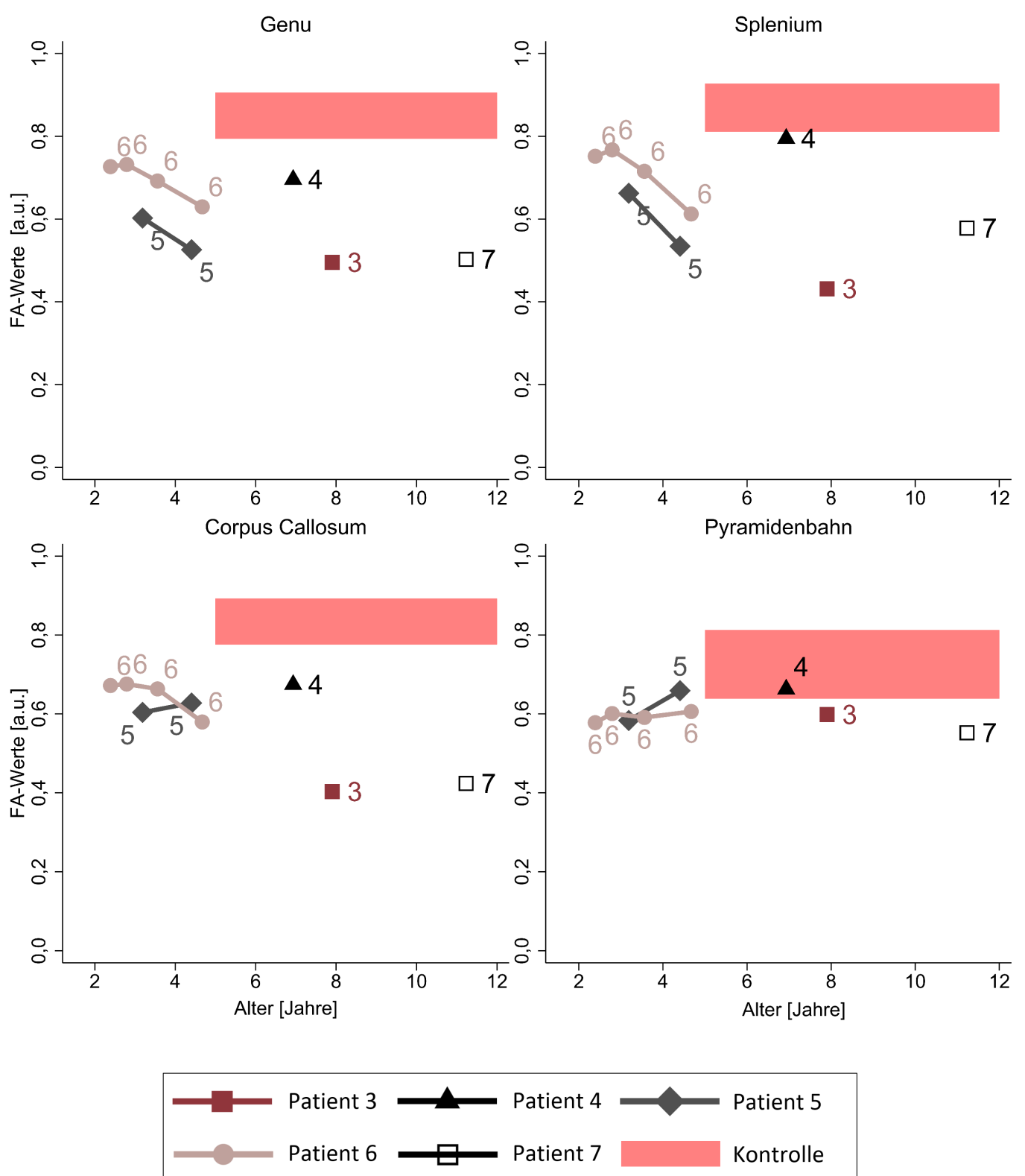

Abb. 15: FA-Werte der PMS-Patienten 3-7 

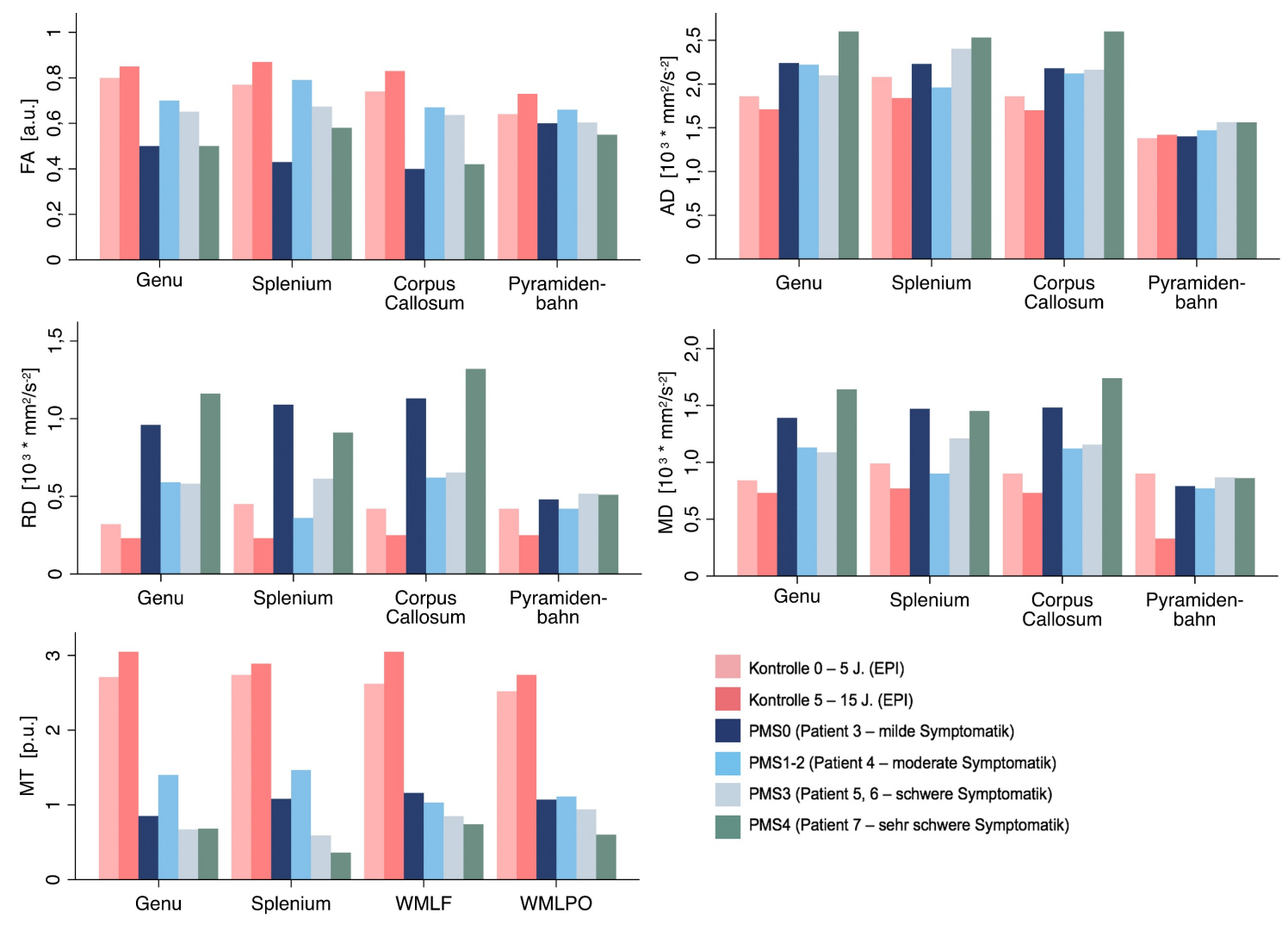

Abb. 16: DT- und MTsat-Werte der PMS-Patienten gestaffelt nach klinischer Symptomatik

Klinischen Einteilung der PMS-Patienten nach dem FDS und MDS. PMS0: Milde Symptomatik. PMS1-2: Moderate Symptomatik. PMS3: Schwere Symptomatik. PMS4: Sehr schwere Symptomatik. Mehrere Messungen in einer Gruppe wurden gemittelt.

\subsection{Axiale Diffusivität}

Die AD-Werte lagen bei den Patienten 3 und 4 in Genu, Splenium und Corpus Callosum im oberen und in der Pyramidenbahn im mittleren Normbereich (s. Abb. 17, s. Tabelle A-4). Bei Patient 5 lagen die AD-Werte in den vier Regionen in der ersten Messung innerhalb des Normbereiches, in der zweiten Messung stieg die AD im Splenium und im Corpus Callosum an und lag in der Folge oberhalb des Normbereiches. Bei den Patienten 5, 6 und 7 wurden die höchsten AD-Werte gemessen. In der Pyramidenbahn lag die AD bei allen fünf Patienten nahe oder im Normbereich.

Ein eindeutiger Zusammenhang zwischen der klinischen Symptomatik der Patienten und der AD zeigte sich nicht, jedoch fiel auf, dass der Patient mit der ausgeprägtesten Klinik die höchste AD aufwies. 

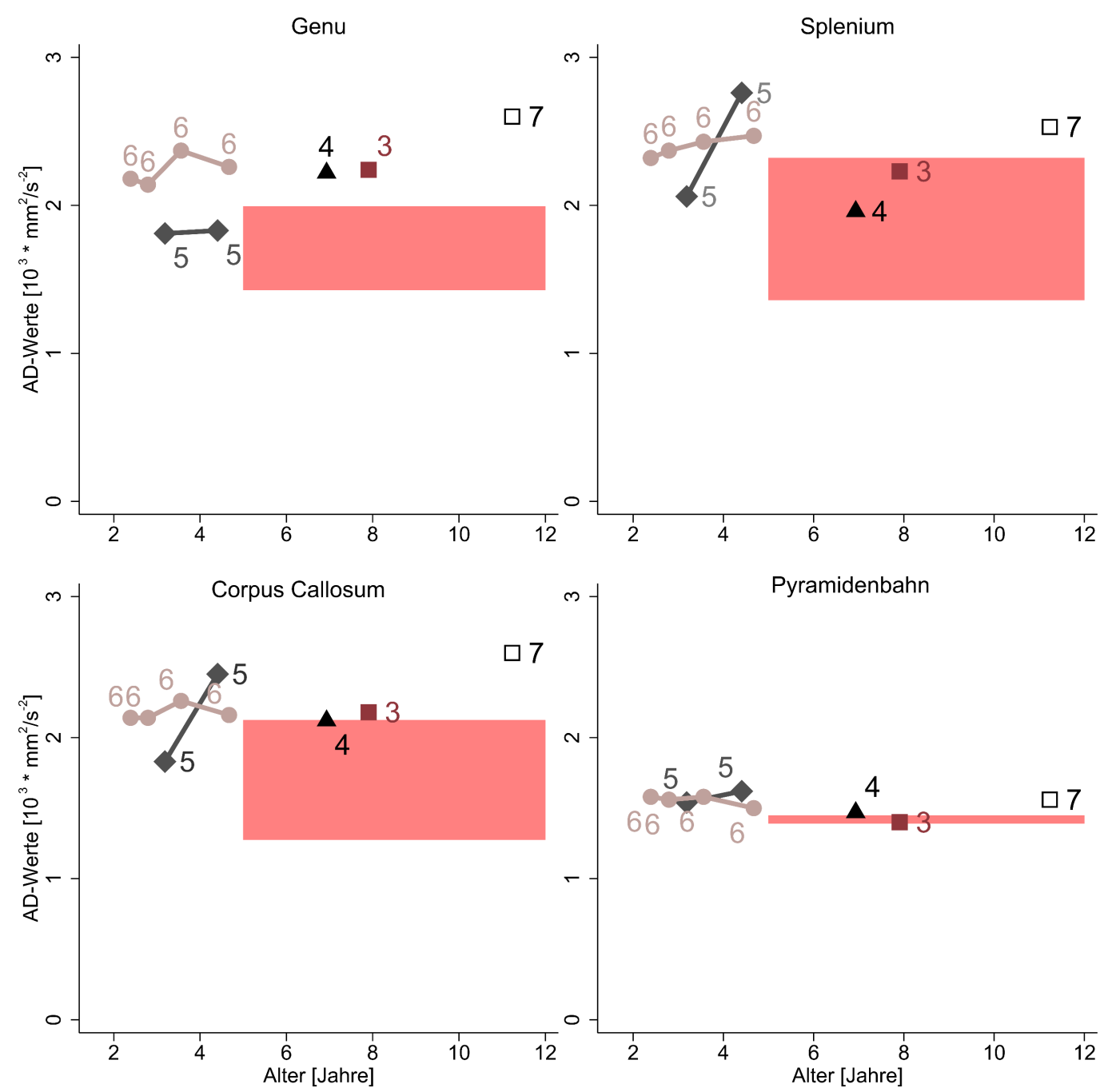

Pyramidenbahn
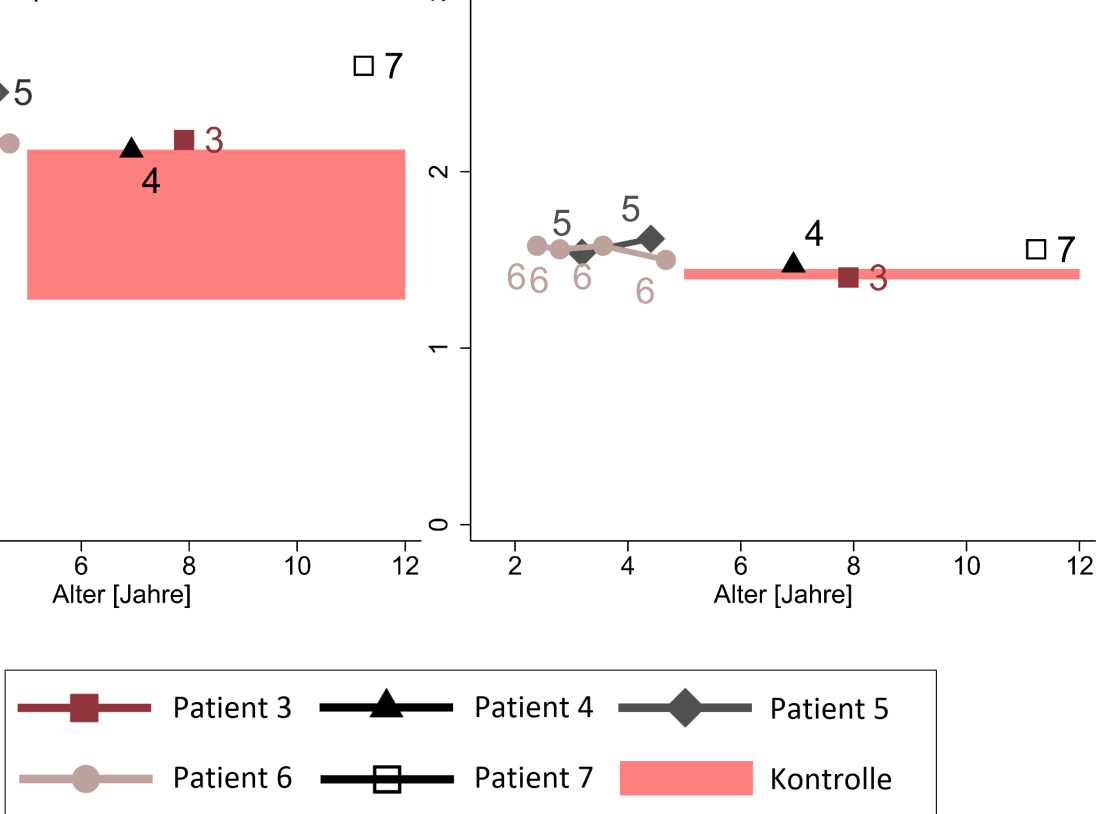

Abb. 17: AD-Werte der PMS-Patienten 3-7

\subsection{Radiale Diffusivität}

In Genu, Splenium und Corpus Callosum waren die RD-Werte bei allen fünf Patienten deutlich oberhalb des Normbereiches (s. Abb. 18, s. Tabelle A-4). In der Pyramidenbahn waren die Werte der Patienten 3 und 4 innerhalb des Normbereiches, die Werte der anderen drei Patienten oberhalb. Bei den Patienten 3 und 7 war die Abweichung zum Normbereich im Genu, im Splenium und im Corpus Callosum am höchsten. Dieses waren die Werte des Patienten mit der mildesten und des Patienten mit der ausgeprägtesten Klinik. Bei den Patienten 5 und 6, von denen mehrere Messungen vorlagen, wurde eine Zunahme der RD mit dem Alter gemessen. Die klinische Symptomatik nahm im gleichen Zeitraum ebenfalls zu (s. Tabelle A-4).

Es wurde ein positiver Zusammenhang zwischen der RD und der Klinik der Patientengruppen PMS1-2, PMS3 und PMS4 und der Kontrolle in Genu, Splenium, Corpus Callosum 
und Pyramidenbahn festgestellt (s. Abb. 16). Je höher die RD war, desto ausgeprägter war die klinische Symptomatik. Die niedrigsten RD-Werte wurden neben der Kontrollgruppe in der Gruppe PMS1-2 (Patient 4) gemessen. Die RD-Werte der Gruppe PMS0 (Patient 3) waren trotz der milderen Klinik deutlich höher als die Werte der Gruppen PMS1-2 und PMS3.
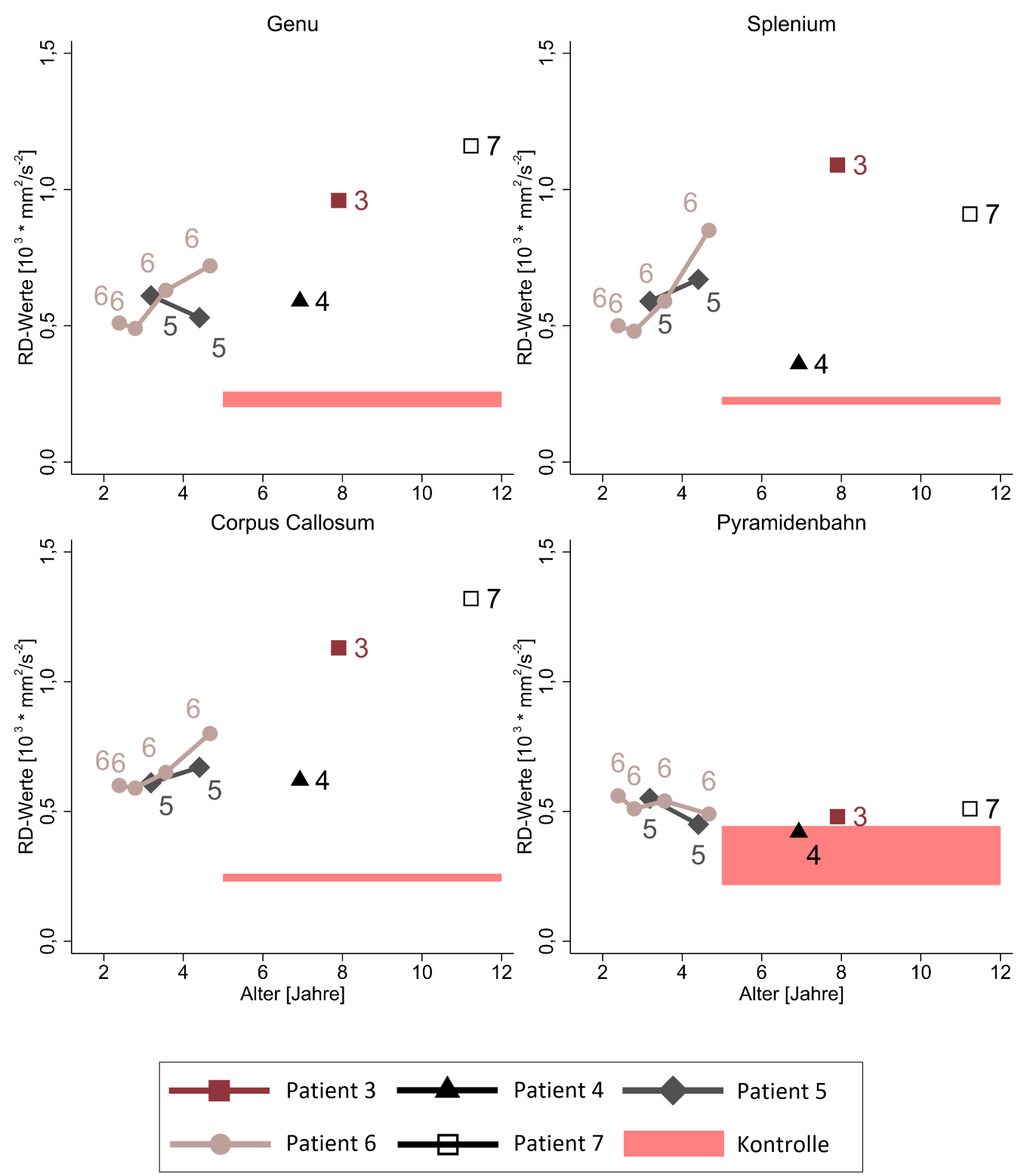

Abb. 18: RD-Werte der PMS-Patienten 3-7

\subsection{Mittlere Diffusivität}

Die MD-Werte lagen bei den fünf Patienten mit PMS in Genu, Splenium und Corpus Callosum oberhalb des Normbereiches (s. Abb. 19, s. Tabelle A-4). In der Pyramidenbahn lagen die MD-Werte der Patienten 5, 6 und 7 oberhalb, die der Patienten 3 und 4 innerhalb des Normbereiches. In den Verlaufsmessungen der Patienten 5 und 6 zeigte sich eine zunehmende MD. Die MD-Werte des Patienten 7 (PMS0) wichen am stärksten von der Kontrollgruppe ab, am wenigsten die der Patienten 4, 5 und 6 (PMS1-2) (s. Abb. 16, 19). Es 
wurde eine Zunahme der MD mit Zunahme der klinischen Symptomatik beobachtet (s. Abb. 16).
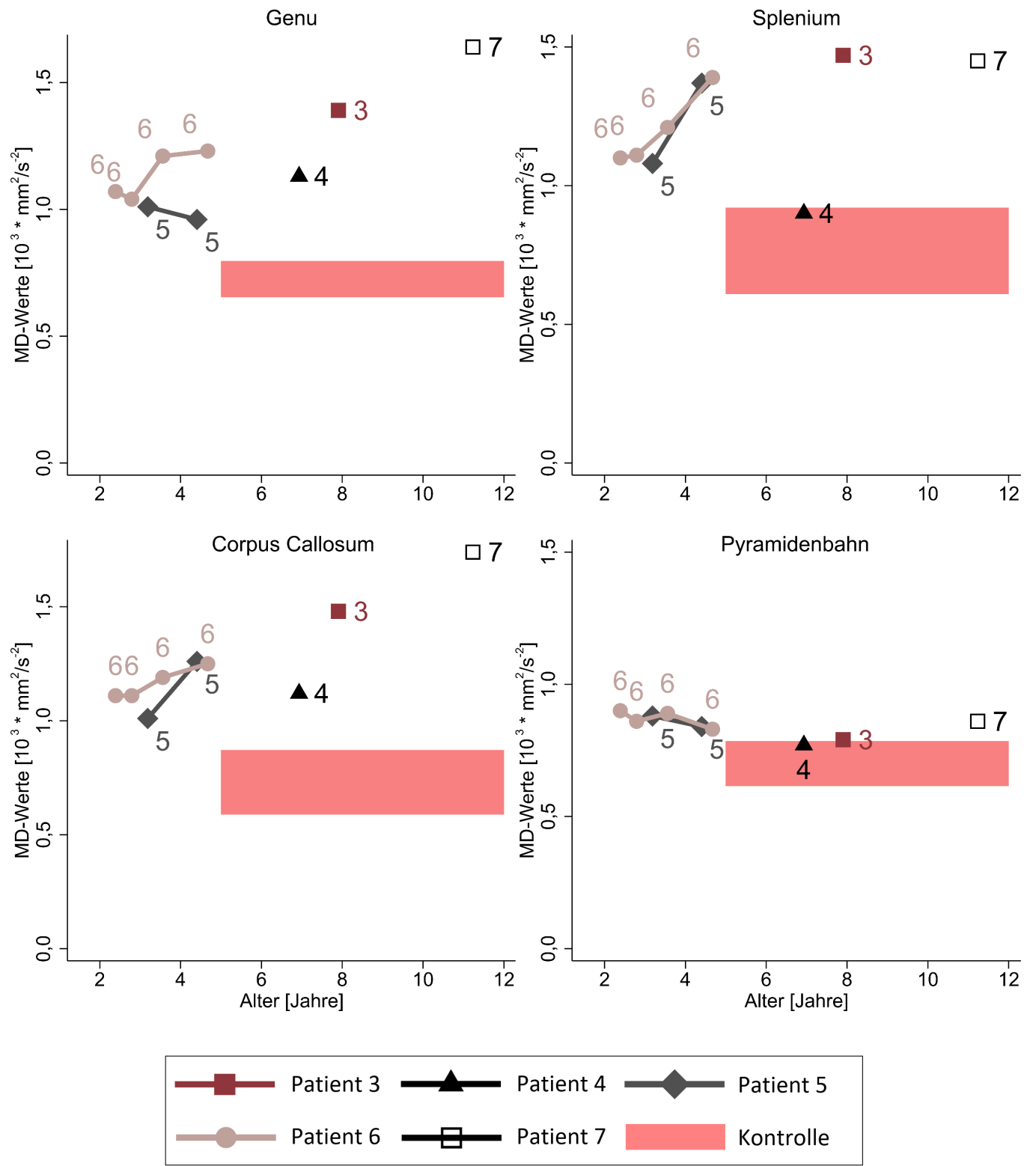

Abb. 19: MD-Werte der PMS-Patienten 3-7

\subsubsection{Verlaufsbeschreibung mittels Magnetisierungstransfer-Bildgebung}

Die MTsat-Werte der Patienten mit PMS lagen in allen Regionen unterhalb des Normbereiches (s. Abb. 20, s. Tabelle A-4). Bei Patient 3 wurde im Verlauf im Genu und im Splenium ein Anstieg der MTsat gemessen, in der WMLF und der WMLPO blieb die MTsat konstant niedrig. Die MTsat nahm bei Patient 4 im Genu und im Splenium zu. In der WMLF und der WMLPO zeigten sich keine Veränderungen. Bei den Patienten 5 und 6 konnte keine ansteigende MTsat in Genu, Splenium WMLF oder WMLPO festgestellt werden.

Sowohl im Genu, im Splenium, in der WMLF, als auch in der WMLPO konnte ein negativer Zusammenhang zwischen der klinischen Symptomatik und der MTsat festgestellt werden 
(s. Abb. 16). Je ausgeprägter die klinische Symptomatik des PMS war, desto niedriger waren die MTsat-Werte in der WM. Lediglich im Genu und im Splenium war die MTsat bei dem Patienten 3 (PMS0) niedriger als bei Patienten 4 (PMS1-2).
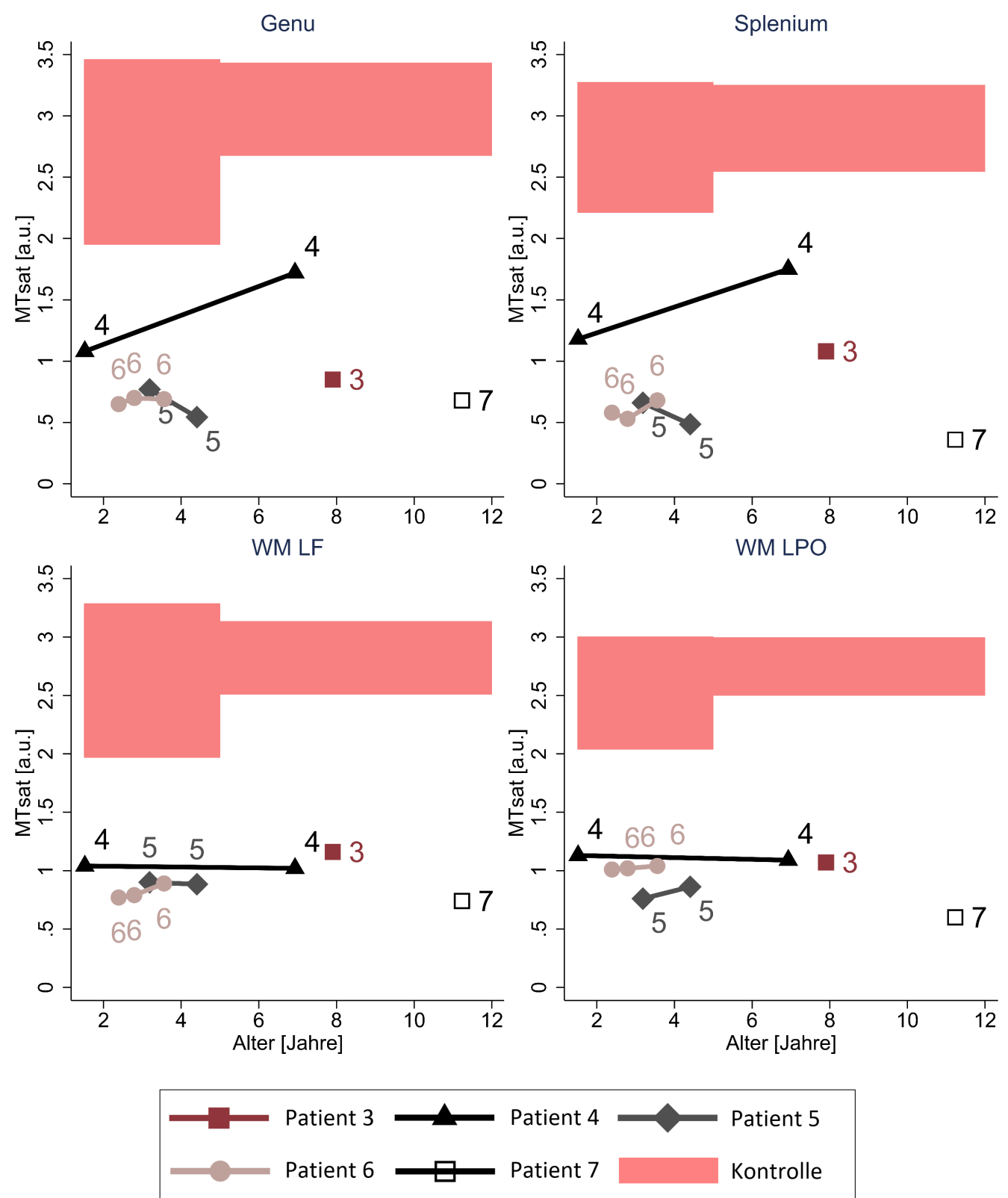

Abb. 20: MTsat-Werte der PMS-Patienten 3-7 


\subsection{Hypomyelinisierung - Zerebrale-Folatdefizienz}

Nachfolgend sind die quantitativen MRT-Daten von fünf Patienten mit cFD im Alter von zwei Monaten bis zwölf Jahren aufgeführt. Die Patienten 8, 9 und 10 sind Geschwister. Untersucht wurden bei allen Patienten die DTI-Parameter FA, AD, RD und MD sowie die MTsat im Genu, Splenium, im gesamten Corpus Callosum, in der WMLF und in der WMLPO. Die ausgeprägteste klinische Symptomatik wies der Patient 8 auf. Unter der Therapie mit Folinsäure verbesserten sich die motorischen und kognitiven Fähigkeiten bei allen cFDPatienten. Bei Patientin 9, die aufgrund des frühen Diagnosezeitpunktes zeitig therapiert werden konnte, kam es zur kompletten Rückbildung der Symptome. Patientin 10 entwickelte, trotz der prädestinierenden FOLR1-Mutation und reduzierter 5-MTHF-Konzentration im CSF zum Diagnosezeitpunkt mit drei Monaten keine Symptome. Die Patientin wurde als einzige von Geburt an mit Folinsäure substituiert.

\subsubsection{Verlaufsbeschreibung der Myelinparameter bei Patient 8}

\subsubsection{Qualitative Beurteilung der MRT-Aufnahmen}

In der ersten MRT-Untersuchung von Patient 8 mit 3,8 Jahren imponierte die WM global hypointens in der T1-w Aufnahme und hyperintens in der T2-w Aufnahme als Zeichen der Hypomyelinisierung (s. Abb. 21). Lediglich im Bereich der Pyramidenbahn, der Sehstrahlung und in Teilen des Corpus Callosums fiel ein Myelinsignal auf (s. Abb. 21). Mit 4,3 Jahren war die Hypomyelinisierung ohne Änderungen in den konventionellen MR-Sequenzen. Die MTsat-Karte wies mit 4,3 Jahren im Gegensatz zur vorherigen Untersuchung global höhere Werte auf. Mit sechseinhalb Jahren zeigte sich in der T1-w und der T2-w Aufnahme sowie in der MTsat-Karte im hinteren Marklager eine Myelinisierung (s. Abb. 21). Parieto-okzipital fielen beidseits ein T1-hypointenses und T2-hyperintenses Areal auf, welches bei dem Patienten als Läsion (Gliose mit Vernarbung) gewertet wurde. Die MTsat war hier niedriger als in der übrigen WM.

Mit zehn Jahren war das Myelindefizit des Patienten im Vergleich zu den gesunden Kontrollen fast aufgeholt. In der T1-w Aufnahme erschien die WM deutlich hyperintenser und in der T2-w hypointenser. In der MTsat-Karte imponierten zugenommene Werte im Bereich der WM, die nun im Normbereich lagen (gelb in der Farbskala) (s. Abb. 21). Die Faserbahnen in der ccFA-Karte wiesen auf eine beinahe normale Neurogenese hin. Die mit sechseinhalb Jahren aufgefallenen parieto-okzipitalen Zonen mit T1-Hypointensität und T2Hyperintensität hatten etwas Größe zugenommen, waren jedoch scharf abgegrenzt (s. Abb. 21). 


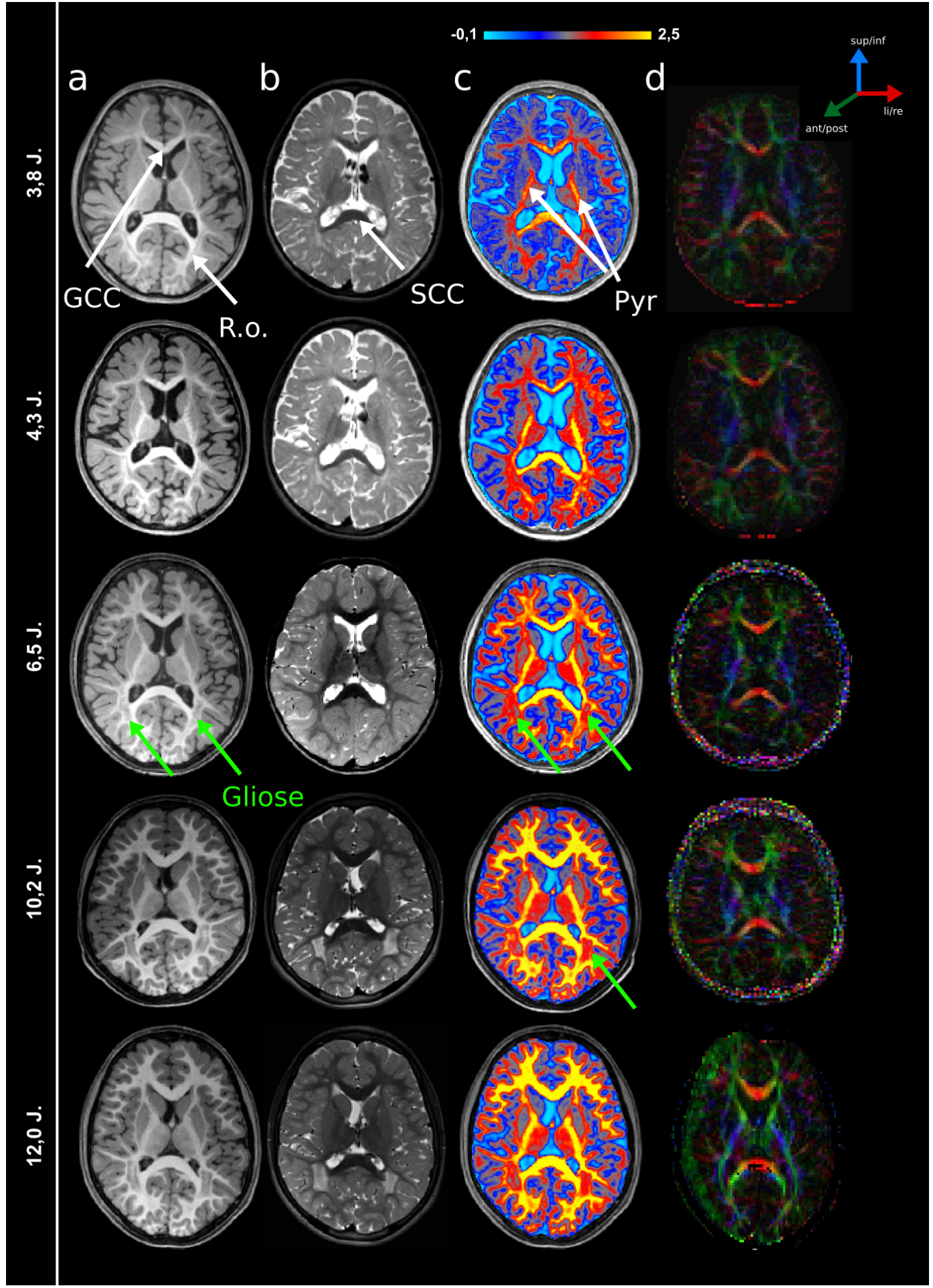

Abb. 21: MRT-Aufnahmen des cFD-Patienten 8

a: axiales T1-w Bild; b: axiales T2-w Bild; c: Koregistrierung der farbkodierten MTsat-Karte auf das korrespondierende axiale T1-w-Bild; d: axiale ccFA-Karte. Weiße Pfeile: Genu (GCC), Splenium (SCC), Pyramidenbahn (Pyr), Sehstrahlung (R.o.). Grüne Pfeile: Okzipitale Gliose

\subsubsection{Verlaufsbeschreibung mittels Diffusionstensor-Bildgebung}

In der DTI wurde ein Anstieg der FA zwischen 3,8 und 5,5 Jahren beobachtet (s. Abb. 22). Eine deutliche Zunahme der FA erfolgte mit 5,5 Jahren. In diesem Zeitraum wurde die intrathekale Folinsäure-Substitution begonnen (s. Abb. 22, grüner Querbalken). Beachtet 
werden muss, dass in den ersten drei Messungen und den letzten fünf STEAMSequenzprotokolle zwei unterschiedlichen Richtungsprotokolle verwendet wurden (vgl. Kapitel 2.2.1).

In der Abb. 22 sind die Messung mit den zwei Protokolle farblich unterschiedlich markiert. Nachdem die FA zwischen dem vierten und sechsten Lebensjahr deutlich stieg, fiel sie in Genu, Splenium und im gesamten Corpus Callosum mit sechseinhalb Jahren zunächst ab (s. Abb. 22). In diesem Zeitraum wurden keine Therapieänderungen durchgeführt. Die nachfolgenden FA-Werte waren wieder im oberen Normbereich (s. Tabelle A-5). Die Parameterwerte der DTI mit zwölf Jahren sind in der Graphik nicht aufgeführt, da die Messung mit einer EPI-Sequenz durchgeführt wurde (s. Abb. 22).

Die AD-Werte lagen vor Beginn der intrathekalen Therapie mit fünf Jahren konstant oberhalb des Normbereiches, mit 5,1 Jahren fiel die AD leicht ab und lag mit 5,5 Jahren schließlich im mittleren Kontrollbereich. Ab fünfeinhalb Jahren fielen keine großen Schwankungen der AD auf. Die RD fiel in den ersten drei Messung vor Beginn der intrathekalen Therapie ausgehend von hohen RD-Werten zunächst ab. Ab dem Alter von fünfeinhalb Jahren lag die RD im Genu, im Splenium und im gesamten Corpus Callosum im unteren Normbereich und einzelne Werte darunter. Der Verlauf der MD zeigte ein ähnliches Bild wie die RD. Zunächst fielen die Werte ab und lagen dann im unteren Normbereich. Mit sieben Jahren waren sowohl die RD- als auch die MD-Werte auf dem niedrigsten Stand. In der letzten Messung lagen sie wieder etwas höher im unteren Normbereich (s. Abb. 22).

\subsubsection{Verlaufsbeschreibung mittels Magnetisierungstransfer-Bildgebung}

Die MTsat nahm ausgehend von Werten zwischen 1 und 2 p. u. in Genu, Splenium und WMLF deutlich mit dem Alter zu (s. Abb. 22). Die MTsat war in Genu und Splenium deutlich höher als in der WMLF und WMLPO. Auffällig war insbesondere der Anstieg zwischen dem vierten und siebten Lebensjahr analog zu den DT-Messungen. Unterschiedliche Messprotokolle wurden bei der MT-Bildgebung nicht angewandt. Mit vier Jahren wurde bei Patienten 8 die Folinsäure-Therapie eingeleitet und mit fünfeinhalb Jahre intrathekal eskaliert. In der WMLF wurde der MTsat-Normbereich nach stetigem Anstieg mit zehn Jahren erreicht. In den Arealen der T2-Hyperintensität in der WMLPO verblieben die Werte konstant unterhalb des Normbereiches. 

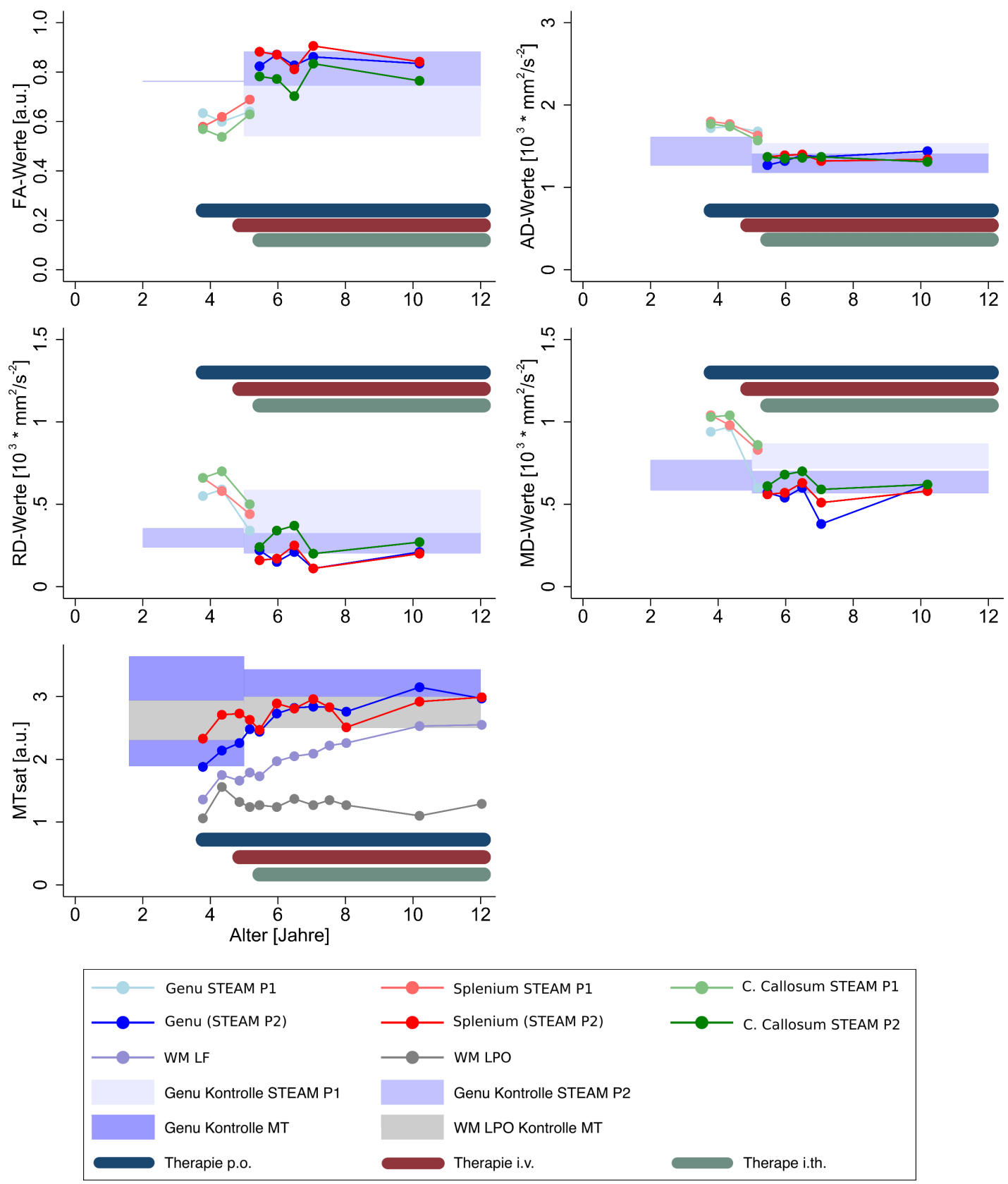

Abb. 22: ROI-Analyse der quantitativen DT- und MT-Parameter des cFD-Patienten 8

FA, AD, RD, MD: Alle DTI-Werte dieser Gruppe mit STEAM-Sequenz aufgenommen (Richtungsprotokolle STEAM_P1 und STEAM_P2). Hellblau: Kontrolle STEAM_P1; Mittelblau: Kontrolle STEAM_P2. Zu beachten: Es wurden Normbereiche des Genu verwendet. Blauer Bereich MTsat: Genu-Kontrollgruppe. Grauer Bereich MTsat: WMLPO-Kontrollgruppe.

\subsubsection{Verlaufsbeschreibung der Myelinparameter bei Patientin 9}

\subsubsection{Qualitative Beurteilung der MRT-Aufnahmen}

Die Patientin 9 zeigte mit zweieinhalb Jahren eine Hypomyelinisierung in den konventionellen MRT-Aufnahmen, die insbesondere okzipital im Subkortex auffiel (s. Abb. 23). Es war eine im Vergleich zur altersentsprechenden Kontrolle vermindert myelinisierte WM (s. Abb. 23) und ein reduzierter Kontrast zwischen GM und WM zu erkennen. In der 
kolorierten MTsat-Karte zeigten sich mit 2,3 Jahren global reduzierte Werte in der WM mit MTsat-Werten zwischen 0 p. u. und 2,5 p. u. (grau-blau bis rot in der Farbskala) (s. Abb. 23). In der Messung mit 3,7 Jahren zeigte sich ein stärkeres Myelin-Signal in der T1-w, der T2-w und der MTsat-Karte (s. Abb. 23). Die Myelinisierung war jedoch weiterhin rückständig. Insbesondere zeigte sich das Defizit in der MTsat-Karte parieto-okzipital und subkortikal. Mit neuneinhalb Jahren zeigten alle MR-Modalitäten eine vollständige Myelinisierung mit T1-hyperintenser und T2-hypointenser WM und MTsat-Werte > 2,5. Das Bild war entsprechend zur altersgleichen gesunden Kontrolle.

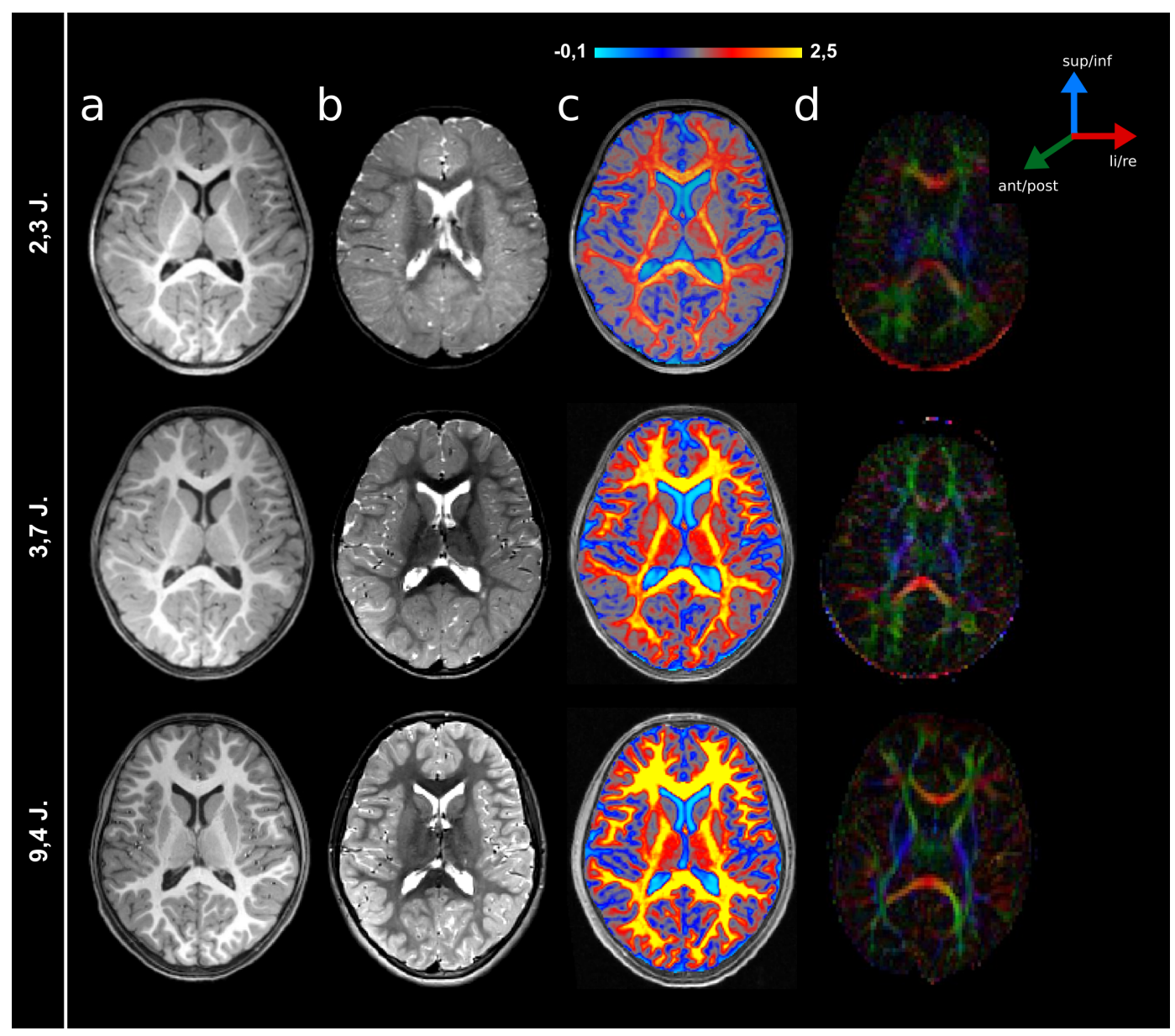

Abb. 23: MRT-Aufnahmen der cFD Patientin 9

a: axiales T1-w Bild; b: axiales T2-w Bild; c: Koregistrierung der farbkodierten MTsat-Karte auf das korrespondierende axiale T1-w-Bild; d: axiale ccFA-Karte.

\subsubsection{Verlaufsbeschreibung der Diffusionstensor-Bildgebung}

Es lagen zwei Messungen mit dem Protokoll STEAM_P1 und acht Messungen mit dem Protokoll STEAM_P2 bei Patientin 9 vor. In den ersten zwei Messungen waren die FAWerte (STEAM_P1), wie auch bei Patient 8, niedriger als in den Messungen der folgenden Untersuchungen mit dem Protokoll STEAM_P2. Die FA lag bis zum dritten Lebensjahr der Patientin unterhalb des Normbereiches, in der Folge durchweg im mittleren und oberen Bereich (s. Abb. 23, Tabelle A-6) Im Splenium nahm die FA von der ersten zur zweiten Messung ab, im Corpus Callosum zu. Die AD, RD und MD lagen in den ersten beiden 
Messungen oberhalb der Kontrollgruppe, mit zunehmendem Alter unter Therapie fielen die Werte ab und lagen im Verlauf am unteren Rand des Normbereiches (s. Abb. 23).

\subsubsection{Verlaufsbeschreibung mittels Magnetisierungstransfer-Bildgebung}

Die MTsat stieg in Genu, Splenium, Corpus Callosum, WMLF und WMLPO im Alter von zwei bis vier Jahren parallel zur Gabe der Folinsäure kontinuierlich an und verblieb ab dem Alter von vier Jahren mit leichten Schwankungen im Normbereich (s. Abb. 23). Dieses war parallel zur klinischen Besserung der Patientin (s. Tabelle 9).
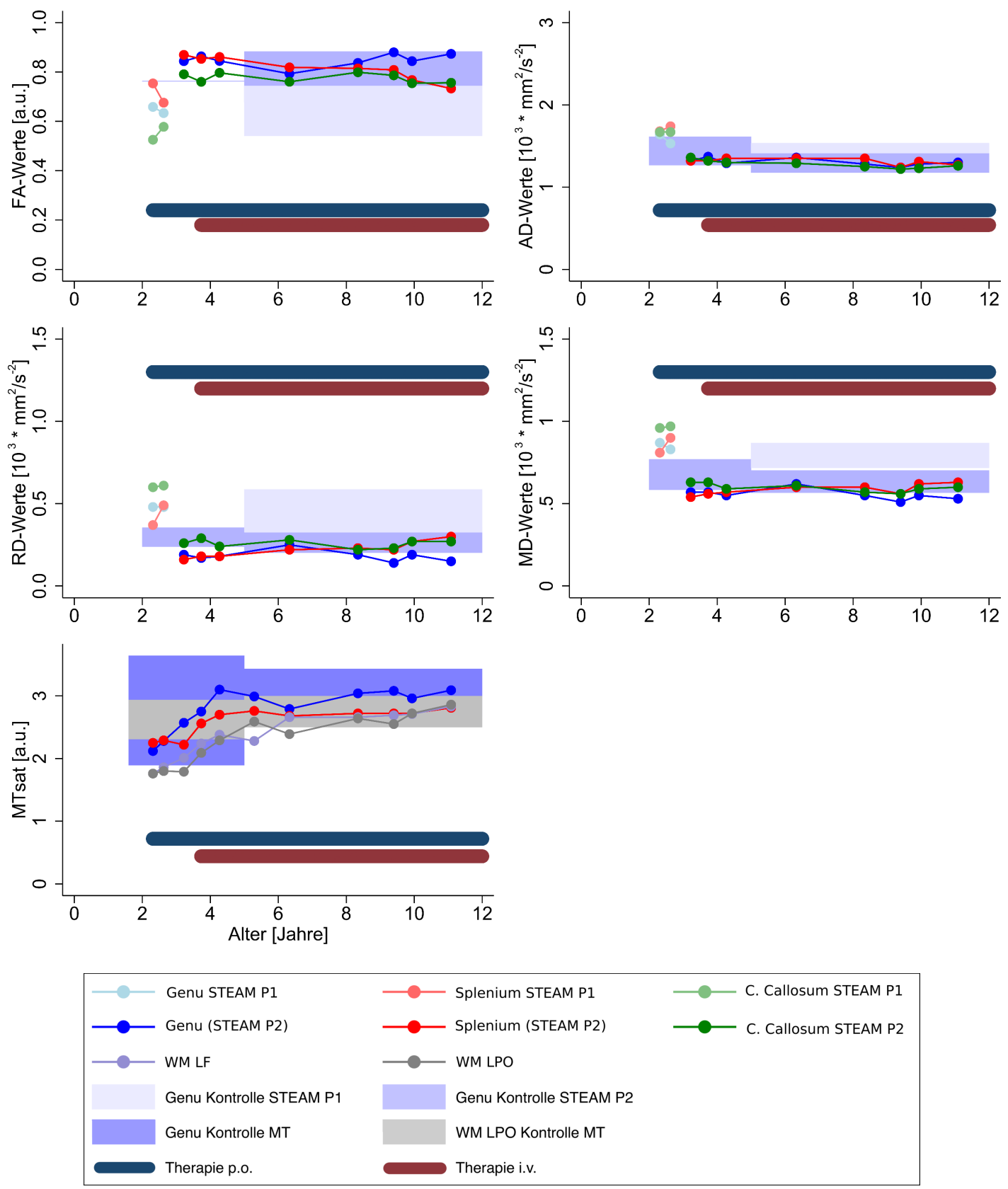

Abb. 24: ROI-Analyse der quantitativen DT- und MT-Parameter der cFD-Patientin 9

FA, AD, RD, MD: Alle DTI-Werte dieser Gruppe mit STEAM-Sequenz aufgenommen (Richtungsprotokolle STEAM_P1 und STEAM_P2). Hellblau: Kontrolle STEAM_P1; Mittelblau: Kontrolle STEAM_P2. Zu beachten: Es wurden Normbereiche des Genu verwendet. Blauer Bereich MTsat: Genu-Kontrollgruppe. Grauer Bereich MTsat: WMLPO -Kontrollgruppe. 


\subsubsection{Verlaufsbeschreibung der Myelinparameter bei Patient 10}

\subsubsection{Qualitative Beurteilung der MRT-Aufnahmen}

In den konventionellen und quantitativen MRT-Aufnahmen zeigte sich die WM zwischen dem zweiten Lebensmonat und dem siebten Lebensjahr bei Patientin 10 entsprechend zur altersgleichen Kontrolle (s. Abb. 25). Es wurde eine physiologisch zunehmende Myelinisierung zwischen dem ersten und dritten Lebensjahr beobachtet. Mit fünfeinhalb Jahren zeigte sich eine auf makroskopischer Ebene vollständige Myelinisierung.

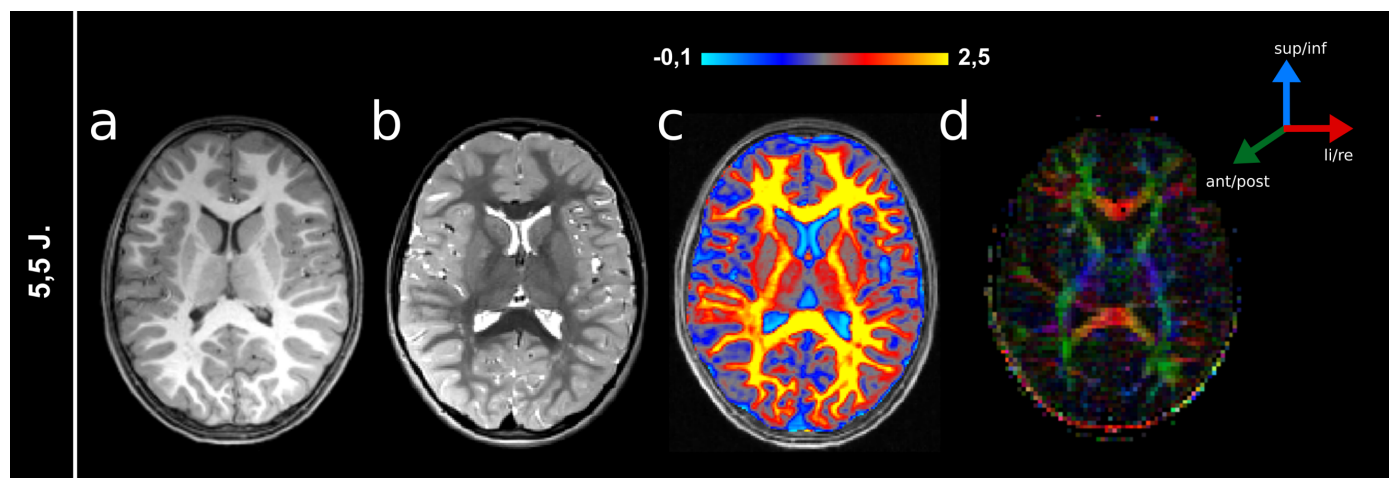

Abb. 25: MRT-Aufnahmen der cFD-Patientin 10

a: axiales T1-w Bild; b: axiales T2-w Bild; c: Koregistrierung der farbkodierten MTsat-Karte auf das korrespondierende axiale T1-w-Bild; d: axiale ccFA-Karte.

\subsubsection{Verlaufsbeschreibung mittels Diffusionstensor-Bildgebung}

Bei der klinisch und bildmorphologisch asymptomatischen Patientin 10 konnte eine Zunahme der FA und Abnahme der RD und MD während der ersten zwei Lebensjahre gemessen werden. Die AD nahm ebenfalls leicht ab (s. Abb. 26, s. Tabelle A-7). Ab dem Alter von 2,3 Jahren lagen die vier DTI-Parameter konstant im Normbereich.

\subsubsection{Verlaufsbeschreibung mittels Magnetisierungstransfer-Bildgebung}

Mit drei Monaten wurde im Genu, Splenium, WMLF und WMLPO eine MTsat unter 1 p. u. gemessen. Zwischen 0,2 und 2,3 Jahren zeigte sich ein Anstieg der MTsat. Ab zwei Jahren lagen alle MTsat-Werte innerhalb des Normbereiches (s. Abb. 26, s. Tabelle A-7). Der deutlichsten Anstieg der MTsat wurde bei der Patientin im ersten Lebensjahr verzeichnet. Die MTsat verdreifachte sich ausgehend vom zweiten Lebensmonat in diesem Zeitraum beinahe. Im Alter von 12 bis 36 Lebensmonate zeigten sich ein langsamerer Anstieg und dem vierten Lebensjahr der Patientin war die MTsat weitgehend konstant. 

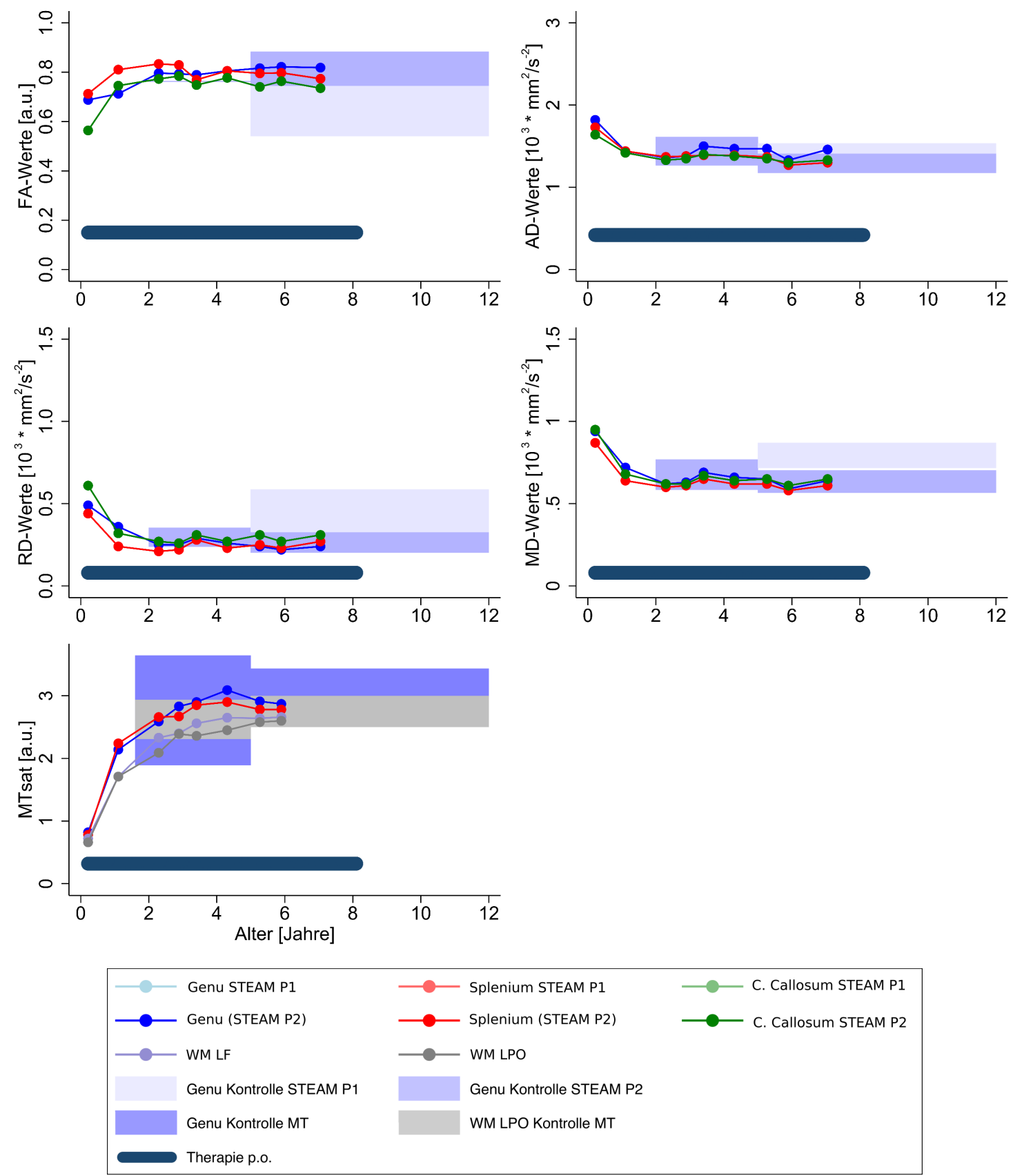

Abb. 26: ROI-Analyse der quantitativen DT- und MT-Parameter der cFD-Patientin 10

FA, AD, RD, MD: Alle DTI-Werte dieser Gruppe mit STEAM-Sequenz aufgenommen (Richtungsprotokolle STEAM_P1 und STEAM_P2). Hellblau: Kontrolle STEAM_P1; Mittelblau: Kontrolle STEAM_P2. Zu beachten: Es wurden Normbereiche des Genu verwendet. Blauer Bereich MTsat: Genu-Kontrollgruppe. Grauer Bereich MTsat: WMLPO-Kontrollgruppe.

\subsubsection{Verlaufsbeschreibung der Myelinparameter bei Patientin 11}

\subsubsection{Qualitative Beurteilung der MRT-Aufnahmen}

In der ersten Messung mit fünf Jahren fiel bei der Patientin eine globale Hypomyelinisierung mit T1-hypointensem und T2-hyperintensem Signal im Bereich der WM, eine Hirnvolumenminderung sowie eine leichte zerebelläre Atrophie mit milder Aufweitung der Hirnfurchen auf (s. Abb. 27) (Cerebellum nicht in Abb. zu sehen). Die MTsat war ubiquitär auf Werte 
zwischen 0 p. u. und 1,5 p. u. reduziert (blau-graue Areale in der Farbskala). Es zeigten einzelne schmale myelinisierte Bereiche mit Werten $>2,5$ p. u. (s. Abb. 27). Mit sechs und sieben Jahren waren weitere Bereiche myelinisiert (s. Abb. 27). Weiterhin fiel jedoch ein Rückstand zur altersgleichen Kontrolle auf (s. Abb. 5).

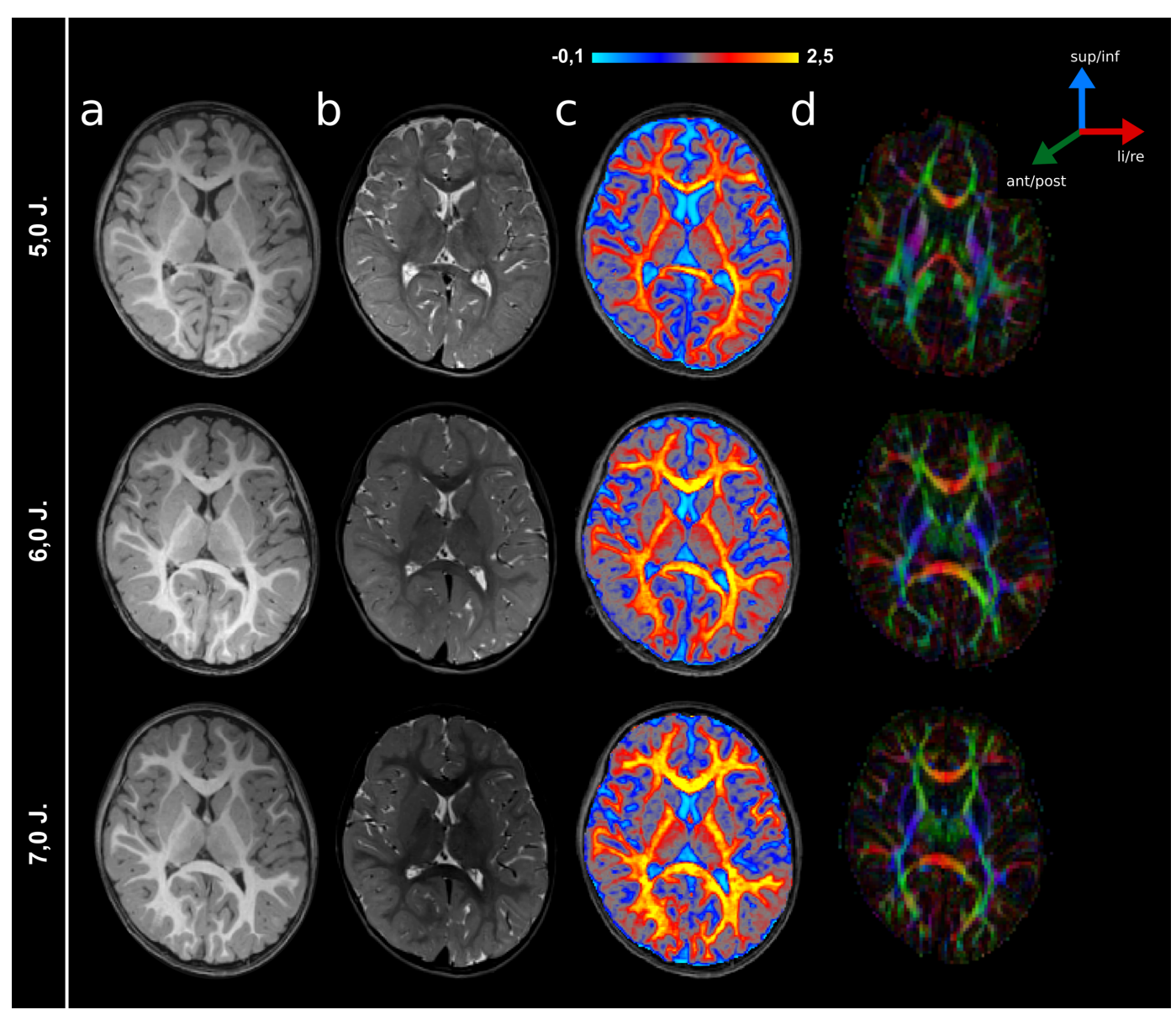

Abb. 27: MRT-Aufnahmen der cFD-Patientin 11

a: axiales T1-w Bild; b: axiales T2-w Bild; c: Koregistrierung der farbkodierten MTsat-Karte auf das korrespondierende axiale T1-w-Bild; d: axiale ccFA-Karte.

\subsubsection{Verlaufsbeschreibung mittels Diffusionstensor-Bildgebung}

Die FA-Werte in Genu, Splenium und Corpus Callosum nahmen ausgehend von Werten am oberen Rand der Kontrollgruppe von der ersten Messung bis zur letzten Messung trotz Besserung der klinischen Symptomatik und Zunahme der im konventionellen MRT sichtbaren makroskopischen Myelinisierung ab (s. Abb. 28, s. Tabelle A-8). Bis auf die FAWerte im Genu und im Splenium mit fünf Jahren und im Corpus Callosum mit sieben Jahren lagen die Werte im Normbereich. Die AD-Werte lagen im oberen Normbereich und zeigten wenig Schwankungen, wohingegen die RD und MD-Werte im Alter von fünf bis sieben Jahren zunahmen und in den letzten Messungen oberhalb des Normbereiches lagen (s. Abb. 28). 
3.4.4.3 Verlaufsbeschreibung mittels Magnetisierungstransfer-Bildgebung

Die MTsat stieg in den vier Regionen im Messzeitraum zwischen dem fünften und siebten Lebensjahr ausgehend von Werten um 2,0 p. u. kontinuierlich an (s. Abb. 28, s. Tabelle A-8). Nachdem die MTsat-Werte zunächst knapp unterhalb des Normbereiches waren, lagen die Werte im Genu und Splenium mit sieben Jahren im Normbereich (s. Abb. 28).
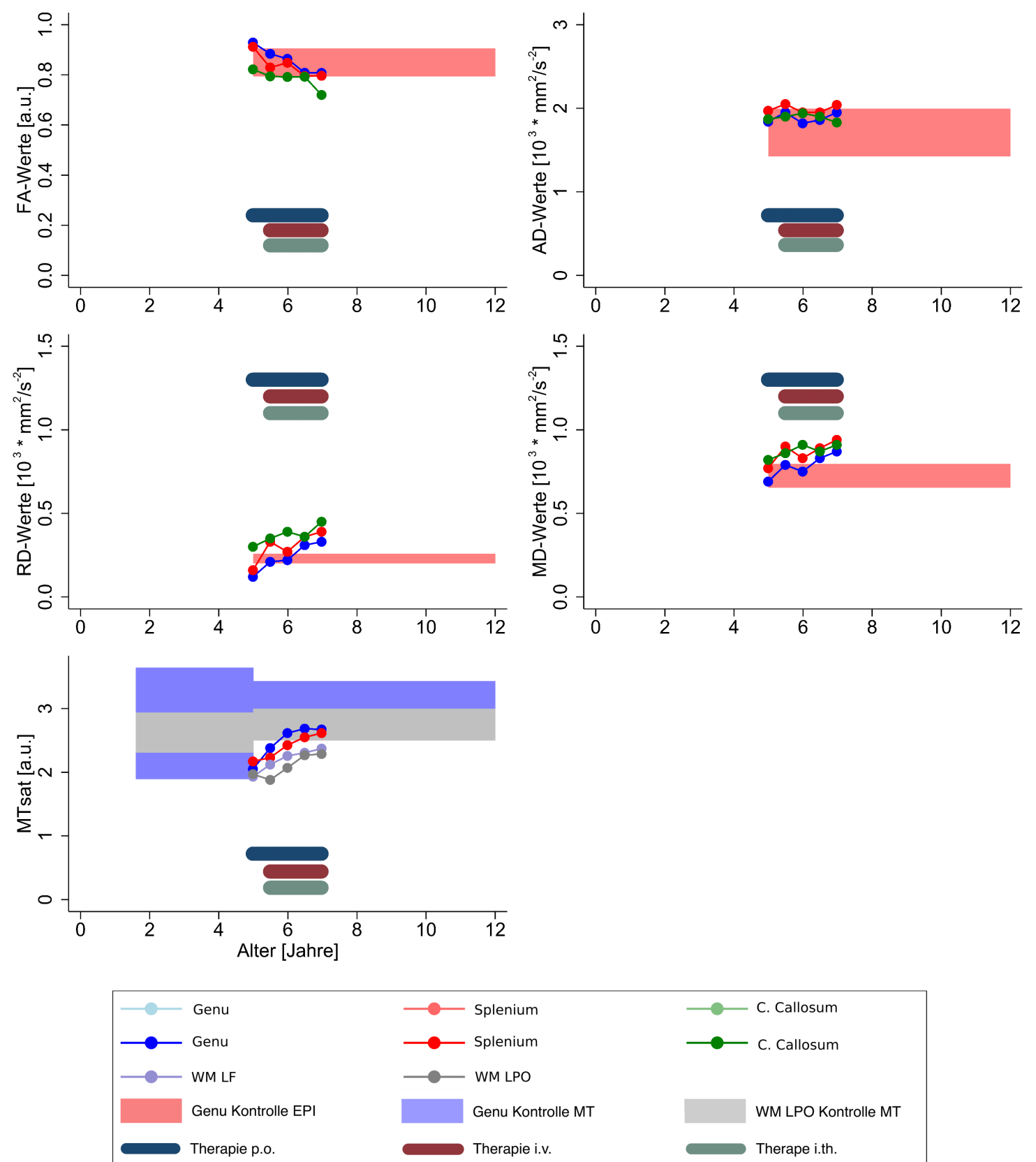

Abb. 28: ROI-Analyse der quantitativen DT- und MT-Parameter der cFD-Patientin 11

FA, AD, RD, MD: Alle DTI-Werte dieser Gruppe mit EPI-Sequenz aufgenommen. Rosa: Normbereich EPI. Zu beachten: Es wurden Normbereiche des Genu verwendet. Blauer Bereich MTsat: Genu-Kontrollgruppe. Grauer Bereich MTsat: WMLPO -Kontrollgruppe. 


\subsubsection{Verlaufsbeschreibung der Myelinparameter bei Patient 12}

\subsubsection{Qualitative Beurteilung der MRT-Aufnahmen}

Patient 12 zeigte mit 1,3 Jahren in den T1-w und T2-w Aufnahmen ein globales Myelindefizit (s. Abb. 29). Die MTsat-Werte waren in der WM stark reduziert (blau-grau in der Farbskala). Mit 2,2 Jahre zeigte sich eine Zunahme der Myelinisierung (s. Abb. 29). Mit fünfeinhalb und sechs Jahren entsprach die Myelinisierung nahezu der von gesunden Kontrollen. In diesem Alter wurde ein altersgerechter klinischer Untersuchungsbefund erhoben und der Patient präsentierte im Gegensatz zu vorherigen Untersuchungen gute motorische und koordinative Fähigkeiten (s. Tabelle 10). 


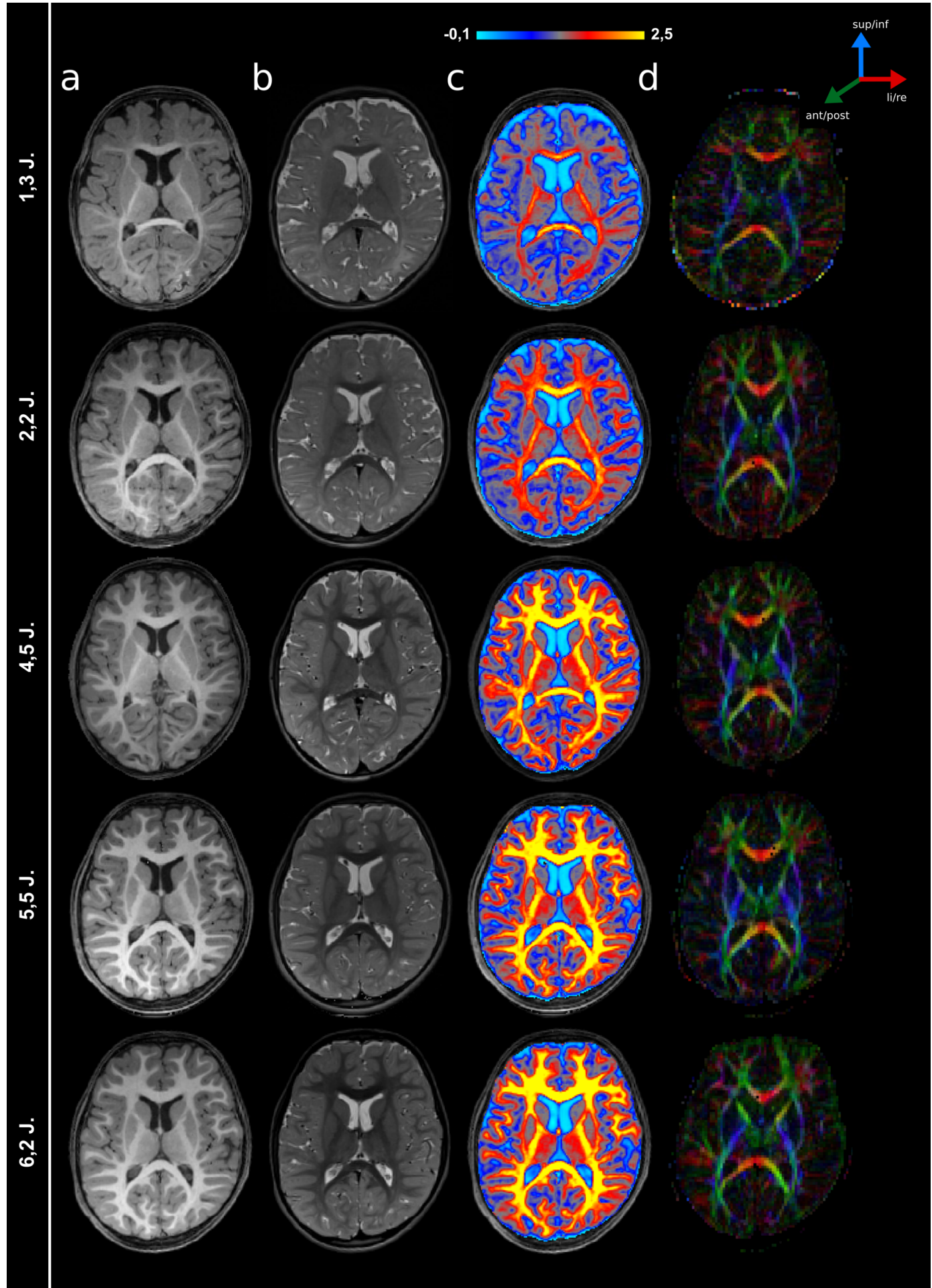

Abb. 29: MRT-Aufnahmen des cFD-Patienten 12

a: axiales T1-w Bild; b: axiales T2-w Bild; c: Koregistrierung der farbkodierten MTsat-Karte auf das korrespondierende axiale T1-w-Bild; d: axiale ccFA-Karte. 
3.4.5.2 Verlaufsbeschreibung der Diffusionstensor-Bildgebung

Die FA lag bei Patient 12 in Genu, Splenium und Corpus Callosum zunächst konstant im unteren Normbereich, fiel dann mit fünf Jahren ab und lagen in der Folge im Splenium und Corpus Callosum etwas unterhalb des Normbereiches (s. Abb. 30, s. Tabelle A-9). Die ADWerte lagen konstant im oberen Normbereich bzw. im Splenium leicht darüber. Die RDund MD-Werte zeigten größere Schwankungen. Zunächst wurden unter Therapie abnehmende RD- und MD-Werte gemessen. Die Werte nahmen mit zweieinhalb Jahren jedoch wieder zu und erreichten vier Jahren nach Therapiebeginn im fünftem Lebensjahr des Patienten ihren höchsten Wert. In der Folge sanken die RD- und MD-Werte im Corpus Callosum wieder ab, waren mit sechseinhalb Jahren jedoch noch leicht oberhalb des Normbereiches.

\subsubsection{Verlaufsbeschreibung mittels Magnetisierungstransfer-Bildgebung}

Die MTsat stieg in Genu, Splenium, WMLF und WMLPO kontinuierlich an, ausgehend von im Vergleich zur Kontrolle niedrigeren MTsat-Werten mit zwei Jahren (s. Abb. 30, s. Tabelle A-9). Im Genu erreichte die MTsat mit dreieinhalb Jahren den Normbereich und im Splenium und in der WMLF mit viereinhalb Jahren. In der WMLPO stieg die MTsat etwas langsamer an. Mit sechs Jahren lagen alle MTsat-Werte im Normbereich (s. Abb. 30). 

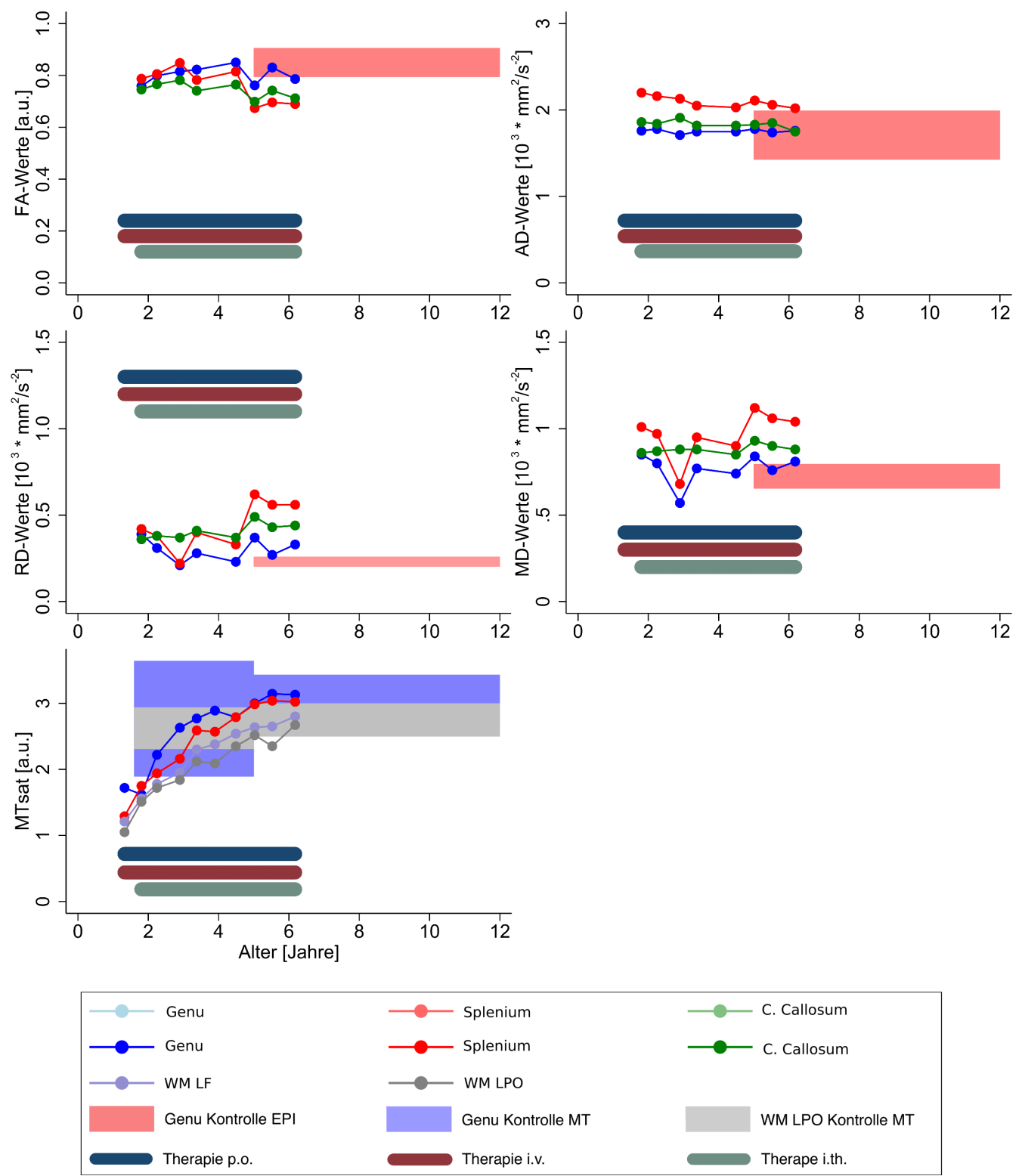

Abb. 30: ROI-Analyse der quantitativen DT- und MT-Parameter des cFD-Patienten 12

FA, AD, RD, MD: Alle DTI-Werte dieser Gruppe mit EPI-Sequenz aufgenommen. Rosa: Normbereich EPI. Zu beachten: Es wurden Normbereiche des Genu verwendet. Blauer Bereich MTsat: Genu-Kontrollgruppe. Grauer Bereich MTsat: WMLPO -Kontrollgruppe. 


\section{Diskussion}

\subsection{Bedeutung der quantitativen Magnetresonanztomographie}

Die konventionelle MRT ist für die Diagnostik und Beurteilung der meisten Myelinisierungsstörungen von unbestrittener Bedeutung. Sie ist jedoch darauf beschränkt makroskopisch morphologische oder fokale Anomalien aufzudecken, die zu regionalen Unterschieden in der Signalintensität der WM, GM oder im CSF führen (Pierpaoli 2010; van der Knaap und Valk 2005). Veränderungen im Bildkontrast aufgrund von mikroskopischen Veränderungen können kaum identifiziert werden oder erweisen sich als sehr unspezifisch (Mohammadi und Weiskopf 2017; van der Voorn et al. 2011).

Die qMRT ermöglicht es mithilfe biophysikalischer Modellierung zahlreiche zusätzliche Eigenschaften wie die longitudinale und transversale Relaxationszeit, Protonendichte, MT oder die Diffusionsbewegung von Wassermolekülen zu erheben (Mohammadi und Weiskopf 2017). Im Gegensatz zur konventionellen MRT werden die Kontrast generierenden Parameter getrennt quantifiziert und Veränderungen dieser sind damit auf einzelne mikrostrukturelle Gewebeveränderungen zurückzuführen (Helms et al. 2010; Mohammadi und Weiskopf 2017). Während die Hyperintensität in T2-w Bildern beispielsweise sowohl durch Ödeme, Demyelinisierung, Dysmyelinisierung, Axonverlust oder Nekrose bedingt sein kann, können DTI oder MT-Bildgebung hier deutlich genauer differenzieren (Pierpaoli 2010).

Die DTI eignet sich u. a. zur Betrachtung mikroskopischer Barrieren, welche die freie Diffusion von Wassermolekülen behindern (Le Bihan und Breton 1985; Mohammadi und Weiskopf 2017). Die DTI-Parameter ermöglichen oder erleichtern die Erkennung von Zellschwellungen im Rahmen von zytotoxischen Ödemen, die Abschätzung der Myelinisierung von Axonen und die Abgrenzung von Faserbahnen (Ertl-Wagner und Wagner 2019; Mohammadi und Weiskopf 2017; Pouwels et al. 2014; Reisert et al. 2014; Song et al. 2002). Mithilfe der DTI sind differenzierte Darstellungen der Nervenverläufe möglich. Die Diffusion verläuft entlang der Nervenfasern sehr gerichtet. Hierfür verantwortlich gelten sowohl Mikrotubuli-assoziierten Proteine in den Axonen, der Axondurchmesser selber, paraaxonale Strukturen als auch die Myelinscheide (Beaulieu 2002; Beaulieu und Allen 1994; Dubois et al. 2014; Le Bihan et al. 2001; Thomsen et al. 1987). In zahlreichen Studien, in denen die Hirnentwicklung untersucht wurde, konnte gezeigt werden, dass während der Myelinisierung die Diffusion radial zu den Axonen und die mittlere Diffusion abnehmen und damit die Diffusionsanisotropie ansteigt (Cascio et al. 2007; Le Bihan et al. 2001; Mukherjee et al. 2008; Neil et al. 1998). Eine hohe FA und eine niedrige RD und MD gelten als Marker für myelinisierte Fasern in der WM (Choudhri et al. 2014; Suzuki et al. 2003), bei strukturellen Veränderung in der WM weichen die Parameter ab (Basser und Jones 2002; Le Bihan et al. 2001; Le Bihan und Breton 1985; van der Knaap und Valk 2005). Zusammenfassend ist die 
DTI ist ein leistungsstarkes Verfahren in sowohl wissenschaftlicher als auch klinischer Anwendung (Mukherjee et al. 2008; Wilke und Dreha-Kulaczewski 2019)

Über Veränderungen des MR-Signals, bedingt durch den Austausch von Magnetisierung zwischen sich frei bewegenden Protonen im Interstitium und den an Makromolekülen der Myelinmembran gebundenen Protonen, ist eine indirekte Beschreibung des Myelingehalts in der MT-Bildgebung möglich (Dreha-Kulaczewski et al. 2012; Helms et al. 2008). Die MTBildgebung weißt gegenüber der konventionellen Bildgebung gut reproduzierbare Werte, Vorteile im Kontrast von GM und WM und zudem eine Unabhängigkeit von T1, lokalem Flipwinkeln, Spulensensitivität und Feldstärke des MR-Tomographen auf (Helms et al. 2008; Matros 2014). Die semiquantitative MTsat-Karten, in denen die prozentuale Reduktion der Magnetisierung mit einem einzelnen MT-Impuls beschrieben wird, haben sich in den letzten Jahren besonders hervorgetan, um Veränderungen der Myelinisierung anzuzeigen (DrehaKulaczewski et al. 2012; Helms et al. 2008).

Das Ziel aktueller Forschung im Bereich der Leukodystrophien ist es, sowohl DTI- als auch MT-Parameter als Biomarker zur Überwachung und Beurteilung von Therapien einzusetzen und damit im klinischen Alltag eine präzisere Darstellung von Krankheitsverläufen und individuellere Therapien zu ermöglichen (Alexander et al. 2011; Pouwels et al. 2014; Wilke und Dreha-Kulaczewski 2019). In dieser Arbeit wurden die DTI-Parameter FA, AD, RD und MD sowie die MTsat bei zwölf Patienten mit den seltenen Erkrankungen ccALD, PMS und cFD im Corpus Callosum, in der Pyramidenbahn sowie in der frontalen und okzipitalen WM retrospektiv analysiert und dabei Zusatzinformationen zu den konventionell-diagnostischen MR-Sequenzen herausgearbeitet.

\subsection{Prädiktionsfähigkeit der Kindlichen-zerebralen-X- chromosomalen-Adrenoleukodystrophie}

Die ccALD ist die schwerste und am schnellsten fortschreitende Verlaufsform der X-ALD (Engelen et al. 2012). In dieser Arbeit wurde der Frage nachgegangen, ob mit der diffusionsgewichteten DTI und der MT-Bildgebung demyelinisierende Läsionen bereits vor der Darstellung in den T1-w oder T2-w MRT-Aufnahmen nachgewiesen werden können. Bekannt ist bereits, dass DTI-Parameter zur Überwachung von Krankheitsverläufen und Auswirkungen von Therapien bei demyelinisierenden Störungen verwendet werden können (Ono et al. 2014; van der Voorn et al. 2011). Es bestätigte sich in dieser Arbeit, dass sowohl die FA und die RD als DTI-Parameter, als auch die MTsat als MT-Parameter sensitiv genug sind, um bereits kleinste demyelinisierte Läsionen in der WM aufzuzeigen. Die genannten quantitativen Parameter waren im Bereich der Läsion bei beiden ccALD-Patienten außerhalb des Kontrollbereiches noch bevor mithilfe der konventionellen MR-Bildgebung die Läsionen bildmorphologischen beschrieben worden sind.

In ROI-basierten Messungen wurden im Bereich der Läsionen bei zwei Patienten mit ccALD verminderte FA-, AD- und MTsat-Werte, sowie erhöhte RD-Werte im Vergleich zur 
Normal-erscheinenden-WM (NAWM, normal appearing white matter) gemessen. In den Bereichen der später detektierten Läsionen waren die FA-Werte bei dem ersten Patienten sechs Monate und bei dem zweiten Patienten zehn Monate vor der Diagnosestellung unterhalb des Normbereiches. Die RD-Werte waren in den Messungen im Areal der Läsion ebenfalls sechs Monate bzw. zehn Monate vor der Diagnosestellung oberhalb des Kontrollbereiches. Die MD war bei einem der zwei Patienten vor der Beschreibung der Läsionen in der konventionellen MR-Bildgebung erhöht.

Pathophysiologisch führt u. a. die bei ccALD zu beobachtende fokale Demyelinisierung zu einem Abbau der Diffusionsbarriere radial zu den Axonen (Ono et al. 2014). Aus diesem Grund können sich die Wassermoleküle in den demyelinisierten Bereichen deutlich isotroper bewegen, was die abnehmende FA und die zunehmende RD widerspiegeln (Mori und Zhang 2006). Ono et al. (2014) räumten in ihrer Studie insbesondere der RD und MD ein hohes Potenzial als Marker zur Früherkennung der ccALD ein. Obgleich sowohl mit DTI als auch mit der MT-Bildgebung nur Korrelationen und keine notwendige Mikrostruktur widergegeben werden, lassen die Parameterveränderungen pathophysiologische Rückschlüsse zu (Mohammadi und Weiskopf 2017).

Bei beiden ccALD-Patienten in dieser Studie war eine Abnahme der AD im Verlauf im Splenium zu beobachten. Die AD gilt als Marker der axonale Integrität (Song et al. 2002). Es ist beschrieben, dass sie während der Myelinisierung zu nimmt und mit zunehmender axonaler Schädigung abnimmt (Hermoye et al. 2006). Bei dem ersten ccALD-Patienten lag die AD im Areal der Läsion unterhalb des Normbereiches bevor radiologisch die Diagnose ccALD gestellt worden ist. Bei dem zweiten Patienten, von dem nur qMRT-Messungen vor der HSZT vorlagen, wurden zum Zeitpunkt der Diagnosestellung AD-Werte im unteren Normbereich gemessen. Zuvor war die AD deutlich gesunken. Da bei ccALD eine axonale Schädigung erst in fortgeschrittenem Stadium beschrieben ist (Ferrer et al. 2010; Fourcade et al. 2014), kann angenommen werden, dass die axonale Schädigung des ersten Patienten weiter als bei dem zweiten Patienten vorangeschritten war und die AD dieses widerspiegelt.

In der ROI-basierten Analyse wurde des Weiteren überprüft, ob die Veränderungen der DTI-Parameter bei demyelinisierenden Läsionen nicht nur direkt in der Läsion, sondern auch in größeren, vorher festgelegten Regionen und Arealen, wie im Splenium superior, Splenium inferior oder im gesamten Splenium darstellbar waren. Bei dem ersten Patienten wurde im gesamten Splenium eine tendenzielle Abnahme der FA und Zunahme der RD und MD über drei Jahre vor der Diagnose der ccALD gemessen. Die Änderungen waren jedoch innerhalb des Kontrollbereiches. Es stellte sich als schwierig dar, daraus einen eindeutigen Hinweis auf eine demyelinisierende Läsion abzuleiten. Bei dem zweiten Patienten konnten in dieser Arbeit keine tendenziellen Veränderungen der FA, AD, RD oder MD im gesamten Splenium oder Splenium superior vor der Diagnosestellung festgestellt werden.

Während im Areal der später detektierten Läsionen deutliche Veränderungen der FA-, RDund MD-Werte schon in früheren Messungen festgestellt worden sind, lagen die 
durchschnittlichen DTI-Parameter im Splenium superior und im gesamten Splenium größtenteils im Normbereich. Vor Diagnosestellung lag bei Patient 1 nur die AD außerhalb dieses. Bei Patient 2 wurde in der Messung zehn Monate vor der HSZT ein oberhalb des Normbereiches liegender AD- und MD-Wert im gesamten Splenium gemessen. Im Splenium superior zeigte sich das gleiche Bild. Als Grund für die weitgehend im Normbereich liegenden quantitativen Parameter in der ROI-basierten Messung im Splenium superior und im gesamten Splenium können die geringen Durchmesser der Läsionen angenommen werden. Nach der HSZT führte die Größenzunahme der Demyelinisierung dazu, dass die absoluten DTI-Werte auch in den größeren Arealen außerhalb des Normbereiches lagen. Als Konsequenz kann gezogen werden, dass, sollten ROI-basierte Analysen in DTI-Daten zur Früherkennung von demyelinisierten Läsionen durchgeführt werden, die Regionen möglichst klein zu halten und anatomische Strukturen beispielsweise in zumindest superior/inferior oder anterior/posterior unterteilt werden sollten.

In der MT-Bildgebung wurden im Bereich der Läsionen zum Zeitpunkt der Diagnosestellung MTsat-Werte deutlich unterhalb des Normbereiches gemessen. Bei dem ersten Patienten waren die MTsat-Werte in der Lokalisation der späteren Läsion bereits sechs Monate vor der HSZT am unteren Rand der Kontrollgruppe, bei dem zweiten Patienten zehn Monate vor der HSZT unterhalb der Normwerte im Splenium. Bei beiden Patienten konnte auch in den Regionen Splenium superior und Gesamt-Splenium eine tendenzielle Abnahme der MTsat ab eineinhalb Jahre vor der Diagnose ccALD beobachtet werden.

Im Alter von viereinhalb Jahren fiel bei Patient 1 eine unscharf begrenzte Veränderung im Splenium in der MTsat-Karte mit MTsat-Werte $<2.5$ p. u. auf. Die verminderten MTsatWerte sind retrospektiv am ehesten als Artefakt zu interpretieren, da in der Folge in dieser Lokalisation keine reduzierten Werte gemessen wurden und die Veränderung in der Karte eine sehr auslaufende, untypische Form aufwies, die nicht auf eine Läsion hindeutete.

Die absoluten qMRT-Parameter-Werte wurden in den Parameter-Karten (maps) mithilfe der Programme Trackvis und FSL dargestellt. Visuell ließen sich die Läsionen in den DTI-Karten aufgrund der niedrigen räumlichen Auflösung nur bedingt darstellen. Die MTsat-Karten waren hier überlegen. In den farbkodierten MTsat-Karten (kodierte Farbskala MTsat -0,1 bis 2,5 p. u.) setzen sich zum Zeitpunkt der Diagnosestellung die demyelinisierten Läsionen mit Werten $<2,5$ p. u. (rot in der kodierten Farbskala) deutlich vom übrigen myelinisierten Splenium mit Werten $>2,5$ p. u. (gelb in der kodierten Farbskala) ab. Die Läsion war bei Patient 2 retrospektiv bereits zehn Monate vor der radiologischen Beschreibung deutlich in der MTsat-Karte zu detektieren. In den konventionellen MRT-Aufnahmen war retrospektiv eine sehr kleine T1-hypointense bzw. T2-hyperintense Veränderungen im Splenium sichtbar. Die Hypo- bzw. Hyperintensität grenzte sich jedoch nur schwach von der NAWM ab. Bei Patient 1 war die Detektion in der verwendeten Farbkodierung vor Diagnosestellung nicht möglich. Wurde die Farbkodierung der MTsat auf -0,1 bis 2,7 p.u. erweitert, konnten die verminderten MTsat-Werte in der Lokalisation der später beschriebenen Läsion auch bei 
Patient 1 vor Diagnosestellung gezeigt werden. Problematisch war jedoch, dass damit auch zahlreiche weitere Bereiche im Splenium und im Truncus mit niedrigeren Werten hervortraten, in denen in der Folge keine Läsionen auftraten. Die leicht verminderten MTsatWerte außerhalb des Läsionsareals entsprachen vermutlich vielmehr der physiologischen Streuung. Der Bereich der Farbkodierung von MTsat-Werten -0,1 bis +2,5 p. u., wie von Dreha-Kulaczewski et al. (2012) für hypomyelinisierende Leukodystrophien vorgeschlagen, erwies sich damit zur Darstellung von demyelinisierenden Läsion als geeignet.

Zusammenfassend zeigte sich, dass die Nutzung von MT-Sequenzen in der klinischen Praxis eine Unterstützung der konventionellen Bildgebung in der Erstdiagnostik von entzündlicher Demyelinisierung bei X-ALD sein kann und Zusatzinformationen zu konventionellen T1-w und T2-w Aufnahmen bietet. Die konventionelle Bildgebung wird weiterhin in der Bildgebenden Diagnostik der ccALD benötigt, jedoch könnten DTI- und MT-Sequenzen die Erkennung von Läsionen erleichtern und unterstreichen. Sollten prospektiv in der Diagnostik DTI- und MTsat-Werte unterhalb des Normbereiches in prädestinierten Arealen bei Patienten mit X-ALD gemessen werden, könnte dieses als Hinweis auf eine beginnende Demyelinisierung gewertet werden. Als Konsequenz könnte daraus die Verkürzung des Abstands zur nächsten Kontrolluntersuchung gezogen werden. Als Vorzüge der MTBildgebung gegenüber der DTI erwiesen sich die höhere räumliche Auflösung und bessere graphische Darstellungsmöglichkeit. Sowohl die DTI- als auch die MTsat-Daten waren intraund interpersonell gut miteinander vergleichbar.

\subsection{Differenzierung von Krankheitsentitäten beim Pelizaeus- Merzbacher-Syndrom}

Das PMS ist eine Erkrankung aus dem Spektrum der PLP1-assozierten hypomyelinisierenden LD. Es weist unterschiedliche phänotypische Erscheinungen auf, von denen einige mit bestimmten Genotypen korrelieren (Hobson und Kamholz 2018; Osório und Goldman 2018). In dieser Arbeit wurde bei Patienten mit klassischem PMS überprüft, ob die quantitative diffusionsgewichtete DTI und die MT-Bildgebung den natürlichen Verlauf der Marklagerveränderungen und ggf. Myelinisierungsvorgängen wiedergeben können und sich daraus mit der klinischen Symptomatik assoziierte Stadien ableiten lassen.

Bei den fünf untersuchten Patienten mit PMS lagen alle FA-, RD-, MD- und MTsat-Werte in Genu, Splenium, im gesamten Corpus Callosum und in der subkortikalen WM deutlich außerhalb des Normbereiches. Die Höhe der DTI-Parameter FA, AD, RD und MD korrelierte zudem in Genu, Splenium und im gesamten Corpus Callosum mit der klinischen Symptomatik bei vier von fünf Patienten. Der MT-Parameter MTsat korrelierte in Genu und Splenium ebenfalls bei vier von fünf Patienten mit der Ausprägung der Symptomatik und in WMLF und WMLPO bei allen fünf Patienten.

Zur Auswertung wurde die PMS-Patienten mithilfe des FDS von Laukka et al. (2013) in die vier Gruppen PMS0 (milde Symptomatik), PMS1-2 (moderate Symptomatik), PMS3 
(schwere Symptomatik) und PMS4 (sehr schwere Symptomatik) eingeteilt. Die Gruppeneinteilung in PMS1-4 war adaptiert an den MDS von Sarret et al. (2016). Obgleich Sarret et al. (2016) in ihrer Arbeit keinen Zusammenhang zwischen Entwicklungsscore und globaler Hypomyelinisierung finden konnten, wurde der Test in dieser Arbeit noch einmal untersucht. Der Zusammenhang zwischen Hypomyelinisierung und klinischer Symptomatik ist zwar umstritten, konnte jedoch in klinischen Studien immer wieder gezeigt werden (Charzewska et al. 2016; Garbern 2007; Inoue 2020; Plecko et al. 2003; Steenweg et al. 2010). Bei Sarret et al. (2016) zeigte sich eine Korrelation der Ausprägung der Hypomyelinisierung nach dem Myelinisierungs-Score von Plecko et al. (2003) und der klinischen Symptomatik nach dem MDS lediglich in WMLF, im vorderen Schenkel der Kapsula interna und in den Bogenfasern. Sarret et al. (2018) konnten in ihrer Studie mit 32 Patienten keinen Zusammenhang zwischen klinischem Schweregrad nach dem MDS und den quantitativen DTI-Parameter in der supratentoriellen WM bei PLP1-mutierten Patienten finden. Sie konnten lediglich eine Zunahme der isotropen Diffusion, also eine Abnahme der FA, in kortikaler und tiefer GM und im infratentoriellen WM mit Zunahme der klinischen Symptomatik zeigen.

In dieser Arbeit konnte in der Gruppe der klinisch moderat, schwer und sehr schwer betroffenen Patienten mit zunehmender Symptomatik (gemessen nach FDS und MDS) in der DTI abnehmende FA- und zunehmende RD- und MD-Werte in Genu, Splenium und in der Pyramidenbahn festgestellt werden. Die MTsat nahm von der Gruppe der Patienten mit milder bis zur Gruppe mit sehr schwerer Symptomatik in Splenium, WMLF und WMLPO kontinuierlich zu. In vernarbten Arealen ohne Myelinisierung wurde, wie zu erwarten, keine Veränderung der quantitativen Parameter festgestellt. Die mit Ausprägung der klinischen Symptomatik zunehmende RD und MD und die in der MT-Bildgebung gemessene Abnahme der MTsat im Marklager spiegelten die Höhe des Myelindefizites wider. Die Ergebnisse der Erhöhung der RD und MD waren in Einklang mit den Erkenntnissen der Studien zu PMS bzw. hypomyelinisierenden LD von Dreha-Kulaczewski et al. (2012) und Laukka et al. (2014) sowie DTI-Studien zu anderen Erkrankungen der WM wie Multipler Sklerose (Ciccarelli et al. 2003), bei Mäusen mit dysmyelinisierter (Song et al. 2002) oder demyelinisierter WM (Song et al. 2005).

Laukka et al. (2014) zeigten bei PMS-Patienten im Vergleich zu Probanden der Kontrollgruppe deutlich erhöhte RD-Werte in ausgewählten WM-Strukturen (vorderer und hinterer Schenkel der Capsula interna, Hirnstiel, Basis der Pons, Genu und Splenium). Dieses führte zu hohen MD- und niedrigen der FA-Werten (Laukka et al. 2014). Steenweg et al. (2010) zeigten in einer Kohorte von 28 Patienten mit verschiedenen hypomyelinisierenden Störungen ebenfalls eine Zunahme der RD in der supratentoriellen WM bei Abnahme der motorischen Fähigkeiten.

Bei den in dieser Arbeit untersuchten PMS-Patienten wurden AD-Werte innerhalb des Normbereiches oder leicht darüber gemessen. Die AD ist bei hypomyelinisierenden LD 
sowohl als reduziert als auch erhöht beschrieben (Klistorner et al. 2015; Laukka et al. 2014; van Rappard et al. 2018). Allgemeinhin ist eine Abnahme der AD mit zunehmender axonaler Schädigung beschrieben (Assaf and Pasternak 2007). Interessanterweise war die AD in Genu und im gesamten Corpus Callosum bei dem Patienten mit der ausgeprägtesten Klinik (Patient 7) am höchsten. Dieses könnte trotz begonnener axonaler Degeneration mit der bei diesen Patienten hohen axonalen Dichte zusammenhängen.

Der MT-Parameter MTsat war in dieser Arbeit bei allen PMS-Patienten erwartungsgemäß reduziert. Grund dafür ist die bei Hypomyelinisierung reduzierte Konzentration der gebundenen Makromoleküle in der fehlenden Myelinmembran. In den MTsat-Karten war das Myelindefizit mit Werte $<1,5$ p. u. bei allen PMS-Patienten graphisch eindeutig abzulesen.

In der Analyse der qMRT-Parameter dieser Arbeit konnte bei allen fünf PMS-Patienten PMS eine Beeinträchtigung des Corpus Callosum, also der Struktur, welche bei Gesunden ausschließlich aus myelinisierten Axonen besteht, gezeigt werden. Bei allen Patienten wurden im Vergleich zur Kontrollgruppe reduzierte FA- und MTsat-Werte und erhöhte RD- und MDWerte im Corpus Callosum gemessen (Sarret et al. 2018). Es konnte damit die Bedeutung der Struktur als potenziell guter morphologischen Marker für die motorische Entwicklung von PMS-Patienten und für das Fortschreiten der Krankheit mit dem Alter, wie von Laukka et al. 2014 und Sarret et al. 2018 beschrieben, bestätigt werden.

Zusammenfassend konnte in dieser Studie gezeigt werden, dass sowohl die absoluten Werte der DTI-Parameter FA, RD, MD als auch des MT-Parameters MTsat eine Einteilung des PMS in Stadien nach der Ausprägung der Myelinisierung ermöglichten. Die Veränderungen der qMRT-Parameter FA, RD, MD und MTsat im Corpus Callosum und in der WMLF und WMLPO korrelierten mit der klinischen Symptomatik in den Gruppe der Patienten PMS12, PMS3 und PMS4 und der Kontrollgruppe. Die MTsat korrelierte als einziger gemessener qMRT-Parameter in allen vier Patientengruppe mit der Symptomatik. Um Grenzwerte für mögliche Stadien der Hypomyelinisierung anhand der qMRT-Parameter festzulegen sind größere Patientengruppen notwendig. Insgesamt zeigte sich die MT-Bildgebung, aufgrund der höheren räumlichen Auflösung und der besseren Darstellungsmöglichkeit, der DTI überlegen. Der FDS erwies sich zur klinischen Beurteilung des PMS von Kindern > 6 Jahren als geeignet. Für Kleinkinder sind weitere Bereiche und Items hinzuzufügen. Eine Ausweitung der abgefragten Testitems wurde in dieser Arbeit initiiert und sollte in zukünftigen Studien evaluiert werden.

\subsection{Therapiemonitoring bei Zerebraler-Folatdefizienz}

Die cFD ist ein klinisch heterogenes, neurometabolisches Krankheitsbild, das durch eine verminderte Konzentration von 5-MTHF im CSF und (in den meisten Fällen) normaler Serum-Folat-Konzentration gekennzeichnet ist (Grapp et al. 2012; Ramaekers et al. 2012). Nach Einstellung einer adäquaten Folinatsubstitution zeigte sich bei den fünf untersuchten 
Patienten mit cFTD und HFM eine Besserung der klinischen Symptomatik. In dieser Arbeit wurde untersucht, ob sich der Verlauf der myelinmodulierenden Folinatsubstitution in den quantitativen DT- und MT-Sequenzen abbilden ließe.

Sowohl in der DTI als auch in der MT-Bildgebung konnte der Verlauf der Myelinisierung dargestellt und ein Zusammenhang der quantifizierten Myelinisierung und der Therapie festgestellt werden. Ausgehend von Werten außerhalb des Normbereiches nahm die FA unter der Folinsäure-Substitution zu, während die RD und MD abnahm. Ebenso stieg die MTsat mit der Myelinisierung an. Die zunehmende Myelinisierung unter der Therapie konnte bei den Patienten sowohl in den konventionellen MRT-Aufnahmen als auch in den qMRTAufnahmen beobachtet werden. Wie zu erwarten, konnten in vernarbten Arealen ohne Myelinisierung keine Veränderungen der quantitativen Parameter gemessen werden.

Die Durchführung von qMRT-Untersuchungen einer asymptomatischen Patientin mit FOLR1-Mutation, beginnend mit dem zweiten Lebensmonat, ermöglichte die retrospektive Analyse von Daten über einen langen Verlauf hinweg. Obwohl die asymptomatische Patientin die gleiche Mutation wie ihre zwei Geschwister aufwies (vgl. Kapitel 2.1.4), zeigte sie weder klinische noch morphologische Auffälligkeiten in der konventionellen oder qMRTBildgebung. Als Grund hierfür hat sich die bei ihr mit dem ersten Lebensmonat begonnene Folinatsubstitution bestätigt (Grapp et al. 2012; Ramaekers et al. 2013; Steinfeld et al. 2009).

Die bei der Patientin zeitgemäße Myelinisierung dokumentierte sich sowohl in den konventionellen T1-w und T2-w Aufnahmen als auch in den DTI- und MT-Studien. Die in der experimentellen pädiatrischen DTI beschriebenen drei Phasen der Veränderungen der Diffusion der Wassermoleküle in der WM (Hermoye et al. 2006; Schneider et al. 2004; Yoshida et al. 2013) konnte bei der asymptomatischen Patientin weitgehend festgestellt werden. Sie umfassen schnelle DTI-Veränderungen in den ersten drei bis sechs Monaten, gefolgt von langsameren Veränderungen bis zum vollendeten zweiten Lebensjahr und eine relative Stabilität der Diffusionsparameter nach 24 Monaten (Yoshida et al. 2013). Die Umwandlungen sind durch die physiologische Reifung der Nervenfasern in diesem Alter erklärt (Yoshida et al. 2013). Für die MTsat liegen solche longitudinalen Daten noch nicht vor, es zeigte sich jedoch eine weitgehende Übereinstimmung zu den DTI-Erkenntnissen. Bei der in dieser Arbeit untersuchten asymptomatischen Patientin nahmen die MTsat-Werte in der gesamten WM in den ersten vier Lebensjahren zu. Der deutlichste Anstieg der MTsat wurde im ersten Lebensjahr verzeichnet. In diesem Zeitraum verdreifachte sich die Höhe der MTsat ausgehend von einer Messung im zweiten Lebensmonat. Zwischen dem ersten und dritten Lebensjahr zeigte sich ein langsamerer Anstieg der MTsat. Ab dem zweiten Lebensjahr lagen die MTsat-Werte im Bereich der doppelten Standardabweichung bei Patienten mit vollständiger Myelinsierung. Ab dem vierten Lebensjahr war die MTsat nahezu konstant.

Bei den vier weiteren Patienten mit cFTD bzw. HFM wurden zum Teil schwere neurologische und bildmorphologische (Myelin-)Defizite festgestellt. Unter Folinat-Substitution 
zeigten sich jedoch sowohl klinisch als auch in der Bildgebung Besserungen. Übereinstimmend mit der in T1-w und T2-w Aufnahmen beschriebenen Zunahme des Myelinvorkommens zeigte sich auch eine Zunahme der MTsat. Bei zwei von vier Patienten stieg darüber die FA unter Folinat-Substitution an und die RD und MD fiel ab.

Bei dem am schwersten betroffenen Patienten 8 war die Myelinisierung mit knapp vier Jahren deutlich verzögert und nahm auch nach Beginn der oralen Substitution nur langsam zu. Nach Eskalation der Therapie auf eine zunächst intravenöse und schließlich intrathekale Gabe zeigte der Patient jedoch einen deutlichen Anstieg der Myelinisierung. Dieses zeigte sich in den konventionellen T1-w und T2-w Aufnahmen und parallel dazu in einem Anstieg der FA, der MTsat und einem Abfall der RD und MD. Zu Bedenken bleibt, dass zum Zeitpunkt der Therapie-Eskalation die DTI Richtungsprotokolle gewechselt wurden. Dieses kann einen Einfluss auf die Veränderung dieser Parameter, nicht jedoch der MTsat, gehabt haben.

Trotz deutlicher Rückbildung der motorischen Symptome, der Ataxie und der epileptischen Krampfanfälle wurde bei der vierten Patientin mit cFTD (Patientin 11) und dem Patienten mit HFM (Patient 12) entgegen der Erwartung eine Zunahme der RD und MD sowie eine Abnahme der FA in der DTI unter der Folinsäure-Therapie beobachtet. Die ansteigende mittlere und radiale Diffusion deutet zunächst auf eine Zunahme der Schädigung der Myelinmembran hin (Soares et al. 2013). In der MT-Bildgebung konnte bei beiden Patienten hingegen der erwartungsgemäß unter Folinat-Substitution ein MTsat-Anstieg gezeigt werden, sprechend für einen zunehmenden Gehalt an Myelin. Die MTsat nahm im Verlauf der Therapie von der ersten zur letzten Messung bei Patientin 11 und Patient 12 kontinuierlich zu. Die entgegen der Erwartung ansteigenden DTI-Parameter RD und MD der zwei Patienten müssen nicht notwendigerweise mit anatomischen Veränderungen in der WM der Patienten begründet sein (Jeurissen et al. 2019; Mohammadi und Weiskopf 2017). Im Endeffekt sollte sich nie auf einzelne Werte verlassen werden, sondern Interpretationen immer auf Grundlage multimodaler und -parametrischer Diagnostik basieren. Die Besserung der neurologisch-motorischen Symptomatik, der Anstieg der MTsat in der gesamten supratentoriellen WM als auch die Zunahme der T1-Hyperintensität und T2-Hypointensität bekräftigen trotz abnehmender RD und MD die Annahme der unter Therapie erwarteten Zunahme der Myelinisierung. In der Regel werden in DTI-Studien größere Patientengruppen verwendet und die Daten verglichen. $\mathrm{Da}$ in dieser Studie lediglich fünf Patienten mit cFD gemessen wurden, könnte es sich bei dem RD- und MD-Anstieg beispielsweise um einen messbedingten Fehler handeln, der sich in der Betrachtung größerer Patientenkollektive ausgleichen würde.

Zusammenfassend konnte sowohl mit der DTI als auch mit der MT-Bildgebung die voranschreitende Myelinisierung gezeigt und damit eine Einschätzung zum Therapieerfolg der Folinat-Substitution mittels qMRT-Parameter gegeben werden. Beide qMRT-Verfahren zeigten eine hohe Myelinsensitivität. Die MTsat als Parameter der MT-Bildgebung zeigte im Vergleich mit den DTI-Parametern insgesamt einen stärkeren Zusammenhang zwischen 
klinischer Symptomatik und der in den konventionellen Aufnahmen zu beobachteten Zunahme der Myelinisierung unter der Therapie.

\subsection{Limitierungen der Arbeit}

Eine Limitierung dieser Arbeit war die kleine Patientengruppe. Bei seltenen Erkrankungen wie der ccALD, dem PMS oder der cFD sind größere Patientengruppen jedoch nur schwer zu erreichen. Die geringe Anzahl gesunder Kontrollen liegt an dem ethischen Problem, bei Kindern aufwendige Diagnostik ohne klinische Konsequenzen durchzuführen. Die Kontrollen in dieser Studie waren zumeist Kinder, bei denen der Verdacht einer Erkrankung zerebraler Strukturen bestand, der sich nach ersten Untersuchungen jedoch nicht bestätigte.

Eine weitere Limitierung war die Anwendung unterschiedlicher Sequenzen (EPI, STEAM) und Richtungsprotokolle innerhalb der DTI-Datensätze. Aus diesem Grund mussten Normwerte nicht nur für unterschiedliche Altersgruppen, sondern auch für unterschiedliche DTI-Sequenzen und Protokolle gebildet werden. Dementsprechend wurden die Kontrollgruppen kleiner. Aufgrund der kleinen Anzahl von Probanden konnten in dieser Arbeit nur zwei Kontrollgruppen gebildet werden.

Die Einzeichnung von ROI in der ccFA-Karte stellte ebenfalls eine Limitierung dar. Das Vorgehen ist in der Literatur jedoch beschrieben und anerkannt (Choudhri et al. 2014; Hermoye et al. 2006), weißt aber den Bias der Einflussnahme der Bildintensität auf die ROIGrenzen auf (Soares et al. 2013). Die Einzeichnung in Karten mit Kolorierung der Voxel nach Hauptdiffusionsrichtung ohne Unterlegung der FA wäre vorteilhaft, war jedoch nicht im Umfang der verwendeten Auswertungssoftware.

\subsection{Relevanz der Ergebnisse und Ausblick}

Bereits etabliert ist die klinische Verwendung einiger qMRT-Verfahren wie die DTI in der Diagnostik von zerebralen Ischämien (hypoxisch-ischämische Enzephalopathien oder Schlaganfälle), in der Beurteilung von intrakraniellen Abszessen und Epidermoiden sowie in der neurochirurgischen Operationsfeldplanung (Ertl-Wagner und Wagner 2019; Wilke und Dreha-Kulaczewski 2019). Zukünftig sollen DTI- und MT-Parameter als Biomarker zur Beurteilung von Therapiewirkungen sowie zur Differenzierung und Klassifizierung unterschiedlicher Verlaufsformen und Phänotypen eingesetzt werden (Alexander et al. 2011). In dieser Arbeit konnte bestätigt werden, dass sowohl die DTI-Parameter als auch die MTsat Defizite im Myelingehalt anzeigen können und ein großes Potenzial haben in der Frühdiagnostik, Klassifikation von Myelindefiziten und Verlaufsbeurteilung bei Patienten mit Erkrankungen der WM eingesetzt zu werden.

Die FA, die RD und die MTsat unterstrichen ihre Bedeutung als myelinsensitive Parameter, die den reduzierten Gehalt an Myelin bei Demyelinisierung frühzeitig bei Patienten mit ccALD aufzeigen können. Die Parameter waren in den Bereichen der Läsion bei beiden 
ccALD-Patienten außerhalb des Kontrollbereiches noch bevor mit der konventionellen MRBildgebung die Läsionen beschrieben worden sind. Retrospektiv konnten bei beiden Patienten zwar auch in den T2-w Karten Hyperintensitäten im Bereich der Läsionen vor der endgültigen Diagnose festgestellt werden, jedoch grenzten sich die Areale deutlich weniger von der NAWM ab. Abweichungen der DTI- oder MTsat-Werte vom Normbereich in prädestinierten Arealen bei Patienten mit X-ALD sollten momentan zwar keine absolute Indikation für beispielsweise eine HSZT sein, jedoch könnten sie Hinweise auf eine beginnende Demyelinisierung geben und daraus als Konsequenz die Verkürzung des Abstands zur nächsten Kontrolluntersuchung gezogen werden.

Die Ausprägung des Myelindefizites beim PMS konnte mithilfe der DTI- und MT-Parameter FA, RD, MD und MTsat eindeutig gezeigt und darüber hinaus in einen Zusammenhang mit der klinischer Symptomatik gebracht werden. In der Gruppe der moderat und schwer klinisch betroffenen Patienten wurden mit zunehmender Symptomatik in der DTI abnehmende FA- und zunehmende RD- und MD-Werte im Corpus Callosum und der Pyramidenbahn gemessen. Die MTsat nahm von der Gruppe der Patienten mit milder bis zur Gruppe mit ausgeprägter Symptomatik zu. Die Korrelation zwischen den absoluten Werten der qMRT-Parameter und der die klinische Symptomatik beschreibenden Scores zeigte, dass eine Einteilung des PMS in Stadien nach der Ausprägung der Myelinisierung möglich ist.

Als guter Verkaufsmarker um die Substitutionstherapie bei cFD zu monitoren, erwies sich die MTsat. Mit zunehmender Myelinisierung konnte bei allen Patienten eine Zunahme der MTsat gezeigt werden, in vernarbtem Gewebe ohne Zunahme der Myelinisierung zeigten sich hingegen erwartungsgemäß keinen Veränderungen. Die FA, RD und MD verhielten sich bei drei der fünf Patienten ebenfalls retrospektiv erwartungsgemäß zur Pathologie, die MTsat nahm bei allen fünf Patienten mit Besserung der klinischen Symptomatik und der in den konventionellen Aufnahmen zu beobachteten Myelinisierung zu.

Insgesamt erleichterten die DTI und die MT-Bildgebung die Differenzierung von demyelinisiertem Fasern und NAWM und ermöglichten eine quantitative Graduierung von Myelindefiziten. Trotz der bisweilen noch aufwendigen Auswertung und längeren Messzeiten kann die Hinzunahme von DTI- und MT-Sequenzen in die Diagnostik und Verlaufsbeurteilung von LD ein Gewinn sein, der die intra- und interpersonelle Vergleichbarkeit bei Myelinisierungsstörung erhöht. Der hohe Kontrast und die hohe räumliche Auflösung der MTsat-Karten erlaubten eine leichtere Differenzierung von demyelinisierenden Läsionen, nicht-myelinisierter WM, myelinisierter WM, GM und Parenchym. Neuere Studien zeigen inzwischen Modelle, in denen die Eigenschaften verschiedener qMRT-Verfahren kombiniert werden (Mohammadi und Weiskopf 2017; Santis et al. 2016). Dieses könnte weitere interessante Erkenntnisse zur Gewebemikrostruktur bieten. 


\section{$5 \quad$ Zusammenfassung}

Als Leukodystrophien werden unabhängig vom Zelltyp, der Gewebskomponente und der pathogenetischen Prozesse monogenetische Erkrankungen bezeichnet, die primär die weiße Hirnsubstanz betreffen. In dieser Arbeit wurden Diffusionstensor (DT)- und Magnetisierungstransfer (MT)-Daten von zwölf Patienten mit drei unterschiedlichen Typen kindlicher Leukodystrophien sowie von 36 Probanden erhoben. Aufgrund der kleinen Patienten- ( $\mathrm{n}=12$ ) und Probandenzahl ( $\mathrm{n}=36$ ) eignete sich eine deskriptive Analyse. Dafür wurden die quantitativen Parameter fraktionale Anisotropie (FA), axiale Diffusivität (AD), radiale Diffusivität (RD), mittlere Diffusivität (MD) und die MT-Sättigung (MTsat) gemessen. Sowohl die DT-Bildgebung als auch die MT-Bildgebung sind experimentell etablierte Techniken zur Charakterisierung der mikrostrukturellen Eigenschaften der WM. Die DT- und MT-Parameter gelten als potenzielle Biomarker für myelin- und axonbedingte Erkrankungen. In dieser Arbeit wurden die in der Magnetresonanztomographie gewonnenen quantitativen Daten mit den konventionellen T1-w und T2-w Aufnahmen sowie der klinischen Symptomatik der Patienten verglichen.

Zum einen wurde untersucht, ob mit der DT- und der MT-Bildgebung demyelinisierende Läsionen bereits vor der Darstellung in den T1-w oder T2-w Aufnahmen bei Kindlicherzerebraler-X-chromosomaler-Adrenoleukodystrophie (ccALD) nachgewiesen werden können. Es konnte bestätigt werden, dass sowohl die FA, die RD und die MD, als auch die MTsat sensitiv genug sind, um bereits kleinste demyelinisierte Läsionen in der weißen Hirnsubstanz anzuzeigen. Im Bereich der Läsionen zeigten sich verminderte FA- und erhöhte RD- Werte sowie verminderte MTsat-Werte. Bei den zwei untersuchten Patienten zeigten sich in der Lokalisation der Läsionen bereits vom Normbereich abweichende DT- und MTsat-Werte bevor die Läsionen in den konventionellen T1-w und T2-w Aufnahmen beschrieben worden sind. Zwar ließ sich retrospektiv die Läsion als Hyperintensität auch in der T2-w Aufnahmen erkennen, die Unterschiede der Intensität zwischen der Normalerscheinenden-weißen-Hirnsubstanz und der demyelinisierten Areale waren jedoch marginal. Am deutlichsten hoben sich die demyelinisierten Areale in der farbkodierten MTsat-Karte (Kodierung der Farbskala MTsat-Werte -0,1 bis 2,5 p. u.) von der Normal-erscheinendenweißen-Hirnsubstanz ab. Die Läsion war bei einem der zwei Patienten mit ccALD in der MTsat-Karte bereits zehn Monate vor der Diagnosestellung sichtbar. Sechs Monate vor der Diagnosestellung wurden bei dem zweiten Patienten ebenfalls reduzierte MTsat-Werte im Bereich der später sichtbaren Läsion gemessen. Die MT-Bildgebung könnte zukünftig eine unterstützende Sequenz in Magnetresonanz-Verlaufsuntersuchungen zur Früherkennung der ccALD darstellen. Reduzierte MTsat-Werte in prädestinierten Arealen könnten bei Patienten mit X-chromosomaler-Adrenoleukodystrophie als Hinweis auf eine beginnende Demyelinisierung gewertet und daraus als Konsequenz die Verkürzung des Abstands zur nächsten Kontrolluntersuchung gezogen werden. 
Zum anderen wurde sich damit befasst, inwiefern der natürliche Verlauf der Myelinisierung bei hypomyelinisierenden Leukodystrophien am Beispiel des Pelizaeus-MerzbacherSyndroms (PMS) mit den quantitativen Sequenzen dargestellt werden kann, und ob sich daraus Stadien ableiten lassen. Es zeigte sich, dass die FA, die RD, die MD und die MTsat im Corpus Callosum und in der frontalen und parieto-okzipitalen weißen Hirnsubstanz bei allen Patienten deutlich außerhalb des Normbereiches lagen. Die Parameter zeigten das prägnante Myelindefizit der Patienten an, das einherging mit einer deutlichen neurologischen Symptomatik. Mithilfe der DT- und MT-Parameter konnte das Myelindefizit quantitativ dargestellt werden. Es konnte eine Korrelation zwischen den Veränderungen der quantitativen DT- und MT-Parameter und der klinischen Symptomatik gemessen mit dem Funktionsbeeinträchtigungs-Score und dem Motorischen-Entwicklungs-Score für PMSPatienten in den Gruppen mit mittlerer, schwerer und sehr schwerer Symptomatik gezeigt werden.

Die dritte Frage, der nachgegangen wurde, war, ob sich der Verlauf der myelinmodulierenden Folinatsubstitution bei Zerebraler-Folatdefizienz (cFD) in den DT- und MT-Sequenzen abbilden ließe. Sowohl in der DT- als auch in der MT-Bildgebung konnte der Verlauf der Myelinentwicklung deutlich dargestellt werden. Unter der Folinsäure-Substitution und klinischen Besserung nahm die FA bei drei der fünf Patienten zu, während die RD und MD abnahmen. In der MT-Bildgebung konnte bei allen fünf Patienten bei erwartungsgemäß unter Folinat-Substitution voranschreitender Myelinisierung ein MTsat-Anstieg gemessen werden. Wie zu erwarten, kam es in vernarbten Arealen ohne Myelinisierung zu keiner Veränderung der quantitativen Parameter.

Mit beiden quantitativen Magnetresonanz-Verfahren ließ sich indirekt der Verlauf der Myelinisierung sowie Myelindefizite aufzeigen. Die DTI und MT-Bildgebung ermöglichten eine Graduierung der Defizite und bei dem Großteil der Patienten zeigte sich ein Zusammenhang zwischen Größe des Myelindefizites und der Ausprägung der klinischen Symptomatik. Im Gegensatz zur konventionellen Magnetresonanztomographie ermöglichten die verwendeten quantitativen Verfahren Vorteile im intra- und interpersonalem Vergleich. Die MT-Bildgebung zeigte gegenüber der DT-Bildgebung eine Überlegenheit in der räumlichen Auflösung und in der graphischen Darstellbarkeit.

Zusammenfassend konnte sowohl die DT- als auch die MT-Bildgebung quantitative Zusatzinformationen in dem untersuchten Patientenkollektiv herausarbeiten. Beide Verfahren unterstrichen ihr Potenzial, eine Unterstützung in der Diagnostik und Verlaufsbeurteilung demyelinisierender und hypomyelinisierender Leukodystrophien sein zu können. 


\section{Literaturverzeichnis}

Al-Baradie RS, Chudary MW (2014): Diagnosis and management of cerebral folate deficiency: A form of folinic acid-responsive seizures. Neurosciences (Riyadh) 19 , 312-316

Alexander AL, Hurley SA, Samsonov AA, Adluru N, Hosseinbor AP, Mossahebi P, Tromp DPM, Zakszewski E, Field AS (2011): Characterization of cerebral white matter properties using quantitative magnetic resonance imaging Stains. Brain Connect 1, 423-446

Aluri S, Zhao R, Lubout C, Goorden SMI, Fiser A, Goldman ID (2018): Hereditary folate malabsorption due to a mutation in the external gate of the proton-coupled folate transporter SLC46A1. Blood Adv 2, 61-68

Assaf Y, Pasternak O (2007): Diffusion Tensor Imaging (DTI)-based White Matter Mapping in Brain Research: A Review. J Mol Neurosci 34, 51-61

Balashova OA, Visina O, Borodinsky LN (2018): Folate action in nervous system development and disease. Dev Neurobiol $\underline{78}, 391-402$

Barkovich AJ (2000): Concepts of myelin and myelination in neuroradiology. AJNR Am J Neuroradiol 21, 1099-1109

Barkovich AJ (2005): Magnetic resonance techniques in the assessment of myelin and myelination. J Inherit Metab Dis $\underline{28}, 311-343$

Basser PJ, Jones DK (2002): Diffusion-tensor MRI: theory, experimental design and data analysis - a technical review. NMR Biomed 15, 456-467

Baumann N, Pham-Dinh D (2001): Biology of oligodendrocyte and myelin in the mammalian central nervous system. Physiol Rev $\underline{81}, 871-927$

Beaulieu C, Allen PS (1994): Water diffusion in the giant axon of the squid: Implications for diffusionweighted MRI of the nervous system. Magn Reson Med 32, 579-583

Beaulieu C (2002): The basis of anisotropic water diffusion in the nervous system-a technical review. NMR Biomed 15, 435-455

Berger J, Forss-Petter S, Eichler FS (2014): Pathophysiology of X-linked adrenoleukodystrophy. Biochimie 98, 135-142

Bezman L, Moser AB, Raymond GV, Rinaldo P, Watkins PA, Smith KD, Kass NE, Moser HW (2001): Adrenoleukodystrophy: Incidence, new mutation rate, and results of extended family screening. Ann Neurol. 49, 512-517

Boespflug-Tanguy O, Labauge P, Fogli A, Vaurs-Barriere C (2008): Genes involved in leukodystrophies: a glance at glial functions. Curr Neurol Neurosci Rep $\underline{8}, 217-229$

Bonkowsky JL, Nelson C, Kingston JL, Filloux FM, Mundorff MB, Srivastava R (2010): The burden of inherited leukodystrophies in children. Neurology $\underline{75}, 718-725$

Cartier N, Aubourg P (2010): Hematopoietic stem cell transplantation and hematopoietic stem cell gene therapy in X-linked adrenoleukodystrophy. Brain pathology 20, 857-862 
Cascio CJ, Gerig G, Piven J (2007): Diffusion tensor imaging: Application to the study of the developing brain. J Am Acad Child Adolesc Psychiatry 46, 213-223

Charzewska A, Wierzba J, Izycka-Swieszewska E, Bekiesinska-Figatowska M, Jurek M, Gintowt A, Klosowska A, Bal J, Hoffman-Zacharska D (2016): Hypomyelinating leukodystrophies - a molecular insight into the white matter pathology. Clin Genet 무, 293-304

Choudhri AF, Chin EM, Blitz AM, Gandhi D (2014): Diffusion tensor imaging of cerebral white matter: Technique, anatomy, and pathologic patterns. Radiol Clin North Am $\underline{52}$, 413-425

Ciccarelli O, Werring DJ, Barker GJ, Griffin CM, Wheeler-Kingshott CAM, Miller DH, Thompson AJ (2003): A study of the mechanisms of normal-appearing white matter damage in multiple sclerosis using diffusion tensor imaging--evidence of Wallerian degeneration. J Neurol 250, 287-292

Desmoulin SK, Hou Z, Gangjee A, Matherly LH (2012): The human proton-coupled folate transporter: Biology and therapeutic applications to cancer. Cancer Biol Ther 13, 1355-1373

Dreha-Kulaczewski SF, Brockmann K, Henneke M, Dechent P, Wilken B, Gartner J, Helms G (2012): Assessment of myelination in hypomyelinating disorders by quantitative MRI. J Magn Reson Imaging $\underline{36}$, 1329-1338

Dubois J, Dehaene-Lambertz G, Kulikova S, Poupon C, Hüppi PS, Hertz-Pannier L (2014): The early development of brain white matter: a review of imaging studies in fetuses, newborns and infants. Neuroscience 276, 48-71

Eichler F, Mahmood A, Loes D, Bezman L, Lin D, Moser HW, Raymond GV (2007): Magnetic resonance imaging detection of lesion progression in adult patients with $\mathrm{X}$-linked adrenoleukodystrophy. Arch Neurol 64, 659-664

Eichler F, Duncan C, Musolino PL, Orchard PJ, Oliveira S de, Thrasher AJ, Armant M, Dansereau C, Lund TC, Miller WP et al. (2017): Hematopoietic stem cell gene therapy for cerebral adrenoleukodystrophy. N Engl J Med 377, 1630-1638

Engelen M, Kemp S, Visser M de, van Geel BM, Wanders RJA, Aubourg P, Poll BT (2012): X-linked adrenoleukodystrophy (X-ALD): Clinical presentation and guidelines for diagnosis, follow-up and management. Orphanet J Rare Dis $\underline{7}, 51$

Ertl-Wagner B, Wagner MW (2019): Magnetresonanztomographie des Gehirns bei Säuglingen und Kleinkindern. Monatsschr Kinderheilkd 167, 813-824

Ferrer I, Aubourg P, Pujol A (2010): General aspects and neuropathology of X-linked adrenoleukodystrophy. Brain Pathol 20, 817-830

Fourcade S, López-Erauskin J, Ruiz M, Ferrer I, Pujol A (2014): Mitochondrial dysfunction and oxidative damage cooperatively fuel axonal degeneration in X-linked adrenoleukodystrophy. Biochimie 98, 143-149

Garbern JY, Cambi F, Tang X-M, Sima AAF, Vallat JM, Bosch EP, Lewis R, Shy M, Sohi J, Kraft G (1997): Proteolipid protein is necessary in peripheral as well as central myelin. Neuron $\underline{19}, 205-218$

Garbern JY (2007): Pelizaeus-Merzbacher disease: genetic and cellular pathogenesis. Cellular and Molecular Life Sciences 64, 50-65 
Goldman SA, Nedergaard M, Windrem MS (2012): Glial progenitor cell-based treatment and modeling of neurological disease. Science $\underline{338}, 491-495$

Gołębiowska M, Gołębiowska B, Beń-Skowronek I (2018): Newest therapeutic options for adrenoleukodystrophy. World Scientific News 108, 133-143

Grapp M, Just IA, Linnankivi T, Wolf P, Lücke T, Häusler M, Gärtner J, Steinfeld R (2012): Molecular characterization of folate receptor 1 mutations delineates cerebral folate transport deficiency. Brain 135, 2022-2031

Helms G, Dathe H, Kallenberg K, Dechent P (2008): High-resolution maps of magnetization transfer with inherent correction for RF inhomogeneity and T1 relaxation obtained from 3D FLASH MRI. Magn Reson Med 으, 1396-1407

Helms G, Dathe H, Dechent P (2010): Modeling the influence of TR and excitation flip angle on the magnetization transfer ratio (MTR) in human brain obtained from 3D spoiled gradient echo MRI. Magn Reson Med 64, 177-185

Hermoye L, Saint-Martin C, Cosnard G, Lee S-K, Kim J, Nassogne M-C, Menten R, Clapuyt P, Donohue PK, Hua K et al. (2006): Pediatric diffusion tensor imaging: Normal database and observation of the white matter maturation in early childhood. Neuroimage 29, 493-504

Hobson GM, Garbern JY (2012): Pelizaeus-merzbacher disease, pelizaeus-merzbacher-like disease 1, and related hypomyelinating disorders. Semin Neurol $\underline{32}$, 62-67

Hobson GM, Kamholz J: PLP1-Related Disorders. In: Adam, M, Ardinger, H, Pagon, R, Wallace, S, Bean, L, Stephens, K, Amemiya, A (Hrsg.): GeneReviews ${ }^{\circledR}$. University of Washington, Seattle 2018 Hoffman-Zacharska D, Mierzewska H, Szczepanik E, Poznański J, Mazurczak T, JakubiukTomaszuk A, Mądry J, Kierdaszuk A, Bal J (2013): The spectrum of PLP1 gene mutations in patients with the classical form of the Pelizaeus-Merzbacher disease. Med Wieku Rozwoj 17, 293-300

Hudson LD, Puckett C, Berndt J, Chan J, Gencic S (1989): Mutation of the proteolipid protein gene PLP in a human X chromosome-linked myelin disorder. Proc Natl Acad Sci U S A 무, 8128-8131

Hurst S, Garbern J, Trepanier A, Gow A (2006): Quantifying the carrier female phenotype in Pelizaeus-Merzbacher disease. Genet Med $\underline{8}, 371-378$

Inoue K: Pelizaeus-Merzbacher Disease: Molecular and Cellular Pathologies and Associated Phenotypes. In: Sango, K, Yamauchi, J, Ogata, T, Susuki, K (Hrsg.): Myelin: Basic and Clinical Advances. Springer, Singapore 2020, 201-216

Jeurissen B, Descoteaux M, Mori S, Leemans A (2019): Diffusion MRI fiber tractography of the brain. NMR Biomed $\underline{32}, 3785$

Klistorner A, Vootakuru N, Wang C, Yiannikas C, Graham SL, Parratt J, Garrick R, Levin N, Masters L, Lagopoulos J et al. (2015): Decoding diffusivity in multiple sclerosis: analysis of optic radiation lesional and non-lesional white matter. PLoS ONE 10 , 114-122

Kronn D, Goldman ID: Hereditary Folate Malabsorption. In: Adam, M, Ardinger, H, Pagon, R, Wallace, S, Bean, L, Stephens, K, Amemiya, A (Hrsg.): GeneReviews ${ }^{\circledR}$. University of Washington, Seattle 2018 
Laukka JJ, Stanley JA, Garbern JY, Trepanier A, Hobson G, Lafleur T, Gow A, Kamholz J (2013): Neuroradiologic correlates of clinical disability and progression in the X-linked leukodystrophy Pelizaeus-Merzbacher disease. J Neurol Sci $\underline{335}, 75-81$

Laukka JJ, Makki MI, Lafleur T, Stanley J, Kamholz J, Garbern JY (2014): Diffusion tensor imaging of patients with proteolipid protein 1 gene mutations. J Neurosci Res 2ㅡ, 1723-1732

Le Bihan D, Breton E (1985): Imagerie de diffusion in-vivo par résonance magnétique nucléaire.

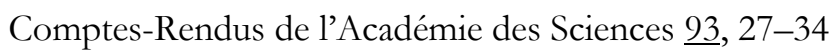

Le Bihan D, Mangin J-F, Poupon C, Clark CA, Pappata S, Molko N, Chabriat H (2001): Diffusion tensor imaging: Concepts and applications. J Magn Reson Imaging 13, 534-546

Le Bihan D, Johansen-Berg H (2012): Diffusion MRI at 25: Exploring brain tissue structure and function. Neuroimage $\underline{61}, 324-341$

Marchetti DP, Donida B, Jacques CE, Deon M, Hauschild TC, Koehler-Santos P, Moura Coelho D de, Coitinho AS, Jardim LB, Vargas CR (2018): Inflammatory profile in X-linked adrenoleukodystrophy patients: Understanding disease progression. J Cell Biochem 119, 1223-1233

Marteyn A, Sarrazin N, Yan J, Bachelin C, Deboux C, Santin MD, Gressens P, Zujovic V, BaronVan Evercooren A (2016): Modulation of the innate immune response by human neural precursors prevails over oligodendrocyte progenitor remyelination to rescue a severe model of PelizaeusMerzbacher disease. Stem Cells $\underline{34}$, 984-996

Matherly LH, Hou Z: Structure and Function of the Reduced Folate Carrier. In: Litwack, G (Hrsg.): Folic acid and folates. (Vitamins and hormones 79). Academic Press, London 2008, 145-184

Matros M: Charakterisierung mikrostruktureller Gewebeveränderungen bei der sporadischen Creutzfeldt-Jakob-Krankheit durch Korrelation von Diffusions- und MagnetisierungstransferBildgebung. Med. Diss. Georg-August-Universität Göttingen 2014

Mohammadi S, Weiskopf N (2017): Computationale Neuroanatomie und Mikrostrukturbildgebung mit der Magnetresonanztomographie. Nervenarzt $\underline{88}, 839-849$

Mori S, Zhang J (2006): Principles of Diffusion Tensor Imaging and Its Applications to Basic Neuroscience Research. Neuron 51, 527-539

Mukherjee P, Chung SW, Berman JI, Hess CP, Henry RG (2008): Diffusion tensor MR imaging and fiber tractography: Technical considerations. AJNR Am J Neuroradiol 29, 843-852

Neil JJ, Shiran SI, McKinstry RC, Schefft GL, Snyder AZ, Almli CR, Akbudak E, Aronovitz JA, Miller JP, Lee BC et al. (1998): Normal brain in human newborns: apparent diffusion coefficient and diffusion anisotropy measured by using diffusion tensor MR imaging. Radiology 209, 57-66

Nowak J, Löbel U, Wölfl M, Schlegel P-G, Warmuth-Metz M (2015): MRI demyelination pattern and clinical course in a child with cerebral X-linked adrenoleukodystrophy (X-ALD). Acta Radiol Open 4, $94-112$

Numata Y, Gotoh L, Iwaki A, Kurosawa K, Takanashi J-I, Deguchi K, Yamamoto T, Osaka H, Inoue K (2014): Epidemiological, clinical, and genetic landscapes of hypomyelinating leukodystrophies. J Neurol 261, 752-758 
O'Neill BP, Moser HW, Saxena KM, Marmion LC (1984): Adrenoleukodystrophy: clinical and biochemical manifestations in carriers. Neurology $\underline{34}, 798-801$

Ono SE, Carvalho Neto Ad, Gasparetto EL, Coelho, Luiz Otávio de Mattos, Escuissato DL, Bonfim CMS, Ribeiro LL (2014): X-linked adrenoleukodystrophy: Correlation between Loes score and diffusion tensor imaging parameters. Radiologia Brasileira 47, 342-349

Osório MJ, Rowitch DH, Tesar P, Wernig M, Windrem MS, Goldman SA (2017): Stem Cell-Based Treatment of Pelizaeus-Merzbacher Disease. Stem Cells $\underline{35}$, 311-315

Osório MJ, Goldman SA (2018): Neurogenetics of Pelizaeus-Merzbacher disease. Handb Clin Neurol 148, $701-722$

Parikh S, Bernard G, Leventer RJ, van der Knaap MS, van Hove J, Pizzino A, McNeill NH, Helman G, Simons C, Schmidt JL et al. (2015): A clinical approach to the diagnosis of patients with leukodystrophies and genetic leukoencephelopathies. Mol Genet Metab Rep 114, 501-515

Pérez-Dueñas B, Ormazábal A, Toma C, Torrico B, Cormand B, Serrano M, Sierra C, Grandis E de, Marfa MP, García-Cazorla A et al. (2011): Cerebral folate deficiency syndromes in childhood: clinical, analytical, and etiologic aspects. Arch Neurol $\underline{68}, 615-621$

Pierpaoli C (2010): Quantitative brain MRI. Top Magn Reson Imaging 21, 63

Plecko B, Stöckler-Ipsiroglu S, Gruber S, Mlynarik V, Moser E, Simbrunner J, Ebner F, Bernert G, Harrer G, Gal A et al. (2003): Degree of hypomyelination and magnetic resonance spectroscopy findings in patients with Pelizaeus Merzbacher phenotype. Neuropediatrics $\underline{34}, 127-136$

Pope S, Artuch R, Heales S, Rahman S (2019): Cerebral folate deficiency: Analytical tests and differential diagnosis. J Inherit Metab Dis, 1-18

Pouwels P, Vanderver A, Bernard G, Wolf NI, Dreha-Kulczewksi SF, Deoni, Sean C. L., Bertini E, Kohlschütter A, Richardson W, ffrench-Constant C et al. (2014): Hypomyelinating leukodystrophies: Translational research progress and prospects. Ann Neurol. 76, 5-19

Qiu A, Jansen M, Sakaris A, Min SH, Chattopadhyay S, Tsai E, Sandoval C, Zhao R, Akabas MH, Goldman ID (2006): Identification of an intestinal folate transporter and the molecular basis for hereditary folate malabsorption. Cell $\underline{127}, 917-928$

Ramaekers V, Sequeira JM, Quadros EV (2012): Clinical recognition and aspects of the cerebral folate

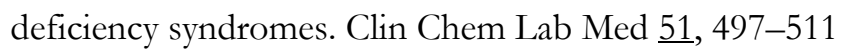

Ramaekers VT, Quadros EV, Sequeira JM (2013): Role of folate receptor autoantibodies in infantile autism. Mol Psychiatry 18, 270-271

Raymond GV, Moser AB, Fatemi A: X-linked adrenoleukodystrophy. In: Adam, M, Ardinger, H, Pagon, R, Wallace, S, Bean, L, Stephens, K, Amemiya, A (Hrsg.): GeneReviews ${ }^{\circledR}$. University of Washington, Seattle 2018

Reisert M, Kiselev VG, Dihtal B, Kellner E, Novikov DS (2014): MesoFT: Unifying diffusion modelling and fiber tracking. Med Image Comput Comput Assist Interv 17, 201-208

Santis S de, Barazany D, Jones DK, Assaf Y (2016): Resolving relaxometry and diffusion properties within the same voxel in the presence of crossing fibres by combining inversion recovery and diffusion-weighted acquisitions. Magn Reson Med 75, 372-380 
Sarret C, Lemaire J-J, Tonduti D, Sontheimer A, Coste J, Pereira B, Feschet F, Roche B, BoespflugTanguy O (2016): Time-course of myelination and atrophy on cerebral imaging in 35 patients with PLP 1-related disorders. Dev Med Child Neurol $\underline{58}, 706-713$

Sarret C, Lemaire J-J, Sontheimer A, Coste J, Savy N, Pereira B, Roche B, Boespflug-Tanguy O (2018): Brain Diffusion Imaging and Tractography to Distinguish Clinical Severity of Human PLP1Related Disorders. Dev Neurosci $\underline{40}, 301-311$

Schiffmann R, van der Knaap MS (2009): An MRI-based approach to the diagnosis of white matter disorders. Neurology $\underline{72}, 750-759$

Schiller S, Henneke M, Gärtner J (2019): Opening New Horizons in the Treatment of Childhood Onset Leukodystrophies. Neuropediatrics $\underline{50}, 211-218$

Schneider JFL, Il'yasov KA, Hennig J, Martin E (2004): Fast quantitative diffusion-tensor imaging of cerebral white matter from the neonatal period to adolescence. Neuroradiology $\underline{46}, 258-266$

Seitelberger F (1995): Neuropathology and genetics of pelizaeus-merzbacher disease. Brain pathology $\underline{5}, 267-273$

Soares J, Marques P, Alves V, Sousa N (2013): A hitchhiker's guide to diffusion tensor imaging. Front. Neurosci $\underline{7}, 31$

Song S-K, Sun S-W, Ramsbottom MJ, Chang C, Russell J, Cross AH (2002): Dysmyelination revealed through MRI as increased radial (but unchanged axial) diffusion of water. Neuroimage 17, 1429-1436 Song S-K, Yoshino J, Le TQ, Lin S-J, Sun S-W, Cross AH, Armstrong RC (2005): Demyelination increases radial diffusivity in corpus callosum of mouse brain. Neuroimage 26, 132-140

Steenweg ME, Vanderver A, Blaser S, Bizzi A, Koning TJ de, Mancini, Grazia M. S., van Wieringen WN, Barkhof F, Wolf NI, van der Knaap M (2010): Magnetic resonance imaging pattern recognition in hypomyelinating disorders. Brain $\underline{133}$, 2971-2982

Steinfeld R, Grapp M, Kraetzner R, Dreha-Kulaczewski S, Helms G, Dechent P, Wevers R, Grosso S, Gärtner J (2009): Folate Receptor Alpha Defect Causes Cerebral Folate Transport Deficiency: A Treatable Neurodegenerative Disorder Associated with Disturbed Myelin Metabolism. Am J Hum Genet $\underline{85}, 354-363$

Sumida K, Inoue K, Takanashi J-I, Sasaki M, Watanabe K, Suzuki M, Kurahashi H, Omata T, Tanaka M, Yokochi K (2016): The magnetic resonance imaging spectrum of pelizaeus-merzbacher disease: A multicenter study of 19 patients. Brain Dev $\underline{38}, 571-580$

Suzuki Y, Matsuzawa H, Nakada T (2003): Feasibility study of single region lambda chart analysis for pyramidal tract physiology. Journal of Neurology 250, 1185-1189

Thomsen C, Henriksen O, Ring P (1987): In Vivo Measurement of Water Self Diffusion in the Human Brain by Magnetic Resonance Imaging. Acta Radiologica 28, 353-361

Uchida N, Chen K, Dohse M, Hansen KD, Dean J, Buser JR, Riddle A, Beardsley DJ, Wan Y, Gong $\mathrm{X}$ et al. (2012): Human neural stem cells induce functional myelination in mice with severe dysmyelination. Sci Transl Med 4, 136-155 
van der Knaap MS, Valk J (Hrsg.): Magnetic Resonance of Myelination and Myelin Disorders, 3. Auflage; Springer, Berlin, Heidelberg 2005

van der Knaap MS, Schiffmann R, Mochel F, Wolf NI (2019): Diagnosis, prognosis, and treatment of leukodystrophies. The Lancet Neurology 18 , 962-972

van der Voorn JP, Pouwels PJW, Powers JM, Kamphorst W, Martin J-J, Troost D, Spreeuwenberg MD, Barkhof F, van der Knaap MS (2011): Correlating quantitative MR imaging with histopathology in X-linked adrenoleukodystrophy. AJNR Am J Neuroradiol 32, 481-489

van Rappard DF, Königs M, Steenweg ME, Boelens JJ, Oosterlaan J, van der Knaap, Marjo S., Wolf NI, Pouwels PJW (2018): Diffusion tensor imaging in metachromatic leukodystrophy. Journal of Neurology 265, 659-668

Verbeek MM, Blom AM, Wevers RA, Lagerwerf AJ, van de Geer J, Willemsen MAAP (2008): Technical and biochemical factors affecting cerebrospinal fluid 5-MTHF, biopterin and neopterin concentrations. Molecular Genetics and Metabolism 25, 127-132

Vogel BH, Bradley SE, Adams DJ, D'Aco K, Erbe RW, Fong C, Iglesias A, Kronn D, Levy P, Morrissey M et al. (2015): Newborn screening for X-linked adrenoleukodystrophy in New York State: Diagnostic protocol, surveillance protocol and treatment guidelines. Mol Genet Metab 114, 599-603

Wang S, Bates J, Li X, Schanz S, Chandler-Militello D, Levine C, Maherali N, Studer L, Hochedlinger K, Windrem M et al. (2013): Human iPSC-derived oligodendrocyte progenitors can myelinate and rescue a mouse model of congenital hypomyelination. Cell Stem Cell $\underline{12}, 252-264$

Watkins D, Schwartzentruber JA, Ganesh J, Orange JS, Kaplan BS, Nunez LD, Majewski J, Rosenblatt DS (2011): Novel inborn error of folate metabolism: identification by exome capture and sequencing of mutations in the MTHFD1 gene in a single proband. J Med Genet $\underline{48}, 590-592$

Wilke M, Dreha-Kulaczewski S (2019): Aktuelle Techniken der Magnetresonanztomographie in der Neuropädiatrie. Monatsschr Kinderheilkd 167, 308-317

Windrem MS, Schanz SJ, Guo M, Tian G-F, Washco V, Stanwood N, Rasband M, Roy NS, Nedergaard M, Havton LA et al. (2008): Neonatal chimerization with human glial progenitor cells can both remyelinate and rescue the otherwise lethally hypomyelinated shiverer mouse. Cell Stem Cell $\underline{2}, 553-565$

Wishnew J, Page K, Wood S, Galvin L, Provenzale J, Escolar M, Gustafson K, Kurtzberg J (2014): Umbilical cord blood transplantation to treat Pelizaeus-Merzbacher Disease in 2 young boys. Pediatrics 134, 1451-7

Woodward K, Palmer R, Rao K, Malcolm S (1999): Prenatal diagnosis by FISH in a family with Pelizaeus-Merzbacher disease caused by duplication of PLP gene. Prenat Diagn 19, 266-268

Yakovlev P, Lecours AR: The myelogenetic cycles of regional maturation of the brain. Regional development of the brain in early life. In: Minkowski A (Hrsg.): Regional Development of the Brain in Early Life: Symposium. Blackwell Science Ltd, Oxford 1967, 3-70

Yoshida S, Oishi K, Faria AV, Mori S (2013): Diffusion tensor imaging of normal brain development. Pediatr Radiol 43, 15-27 
Zhao R, Min SH, Wang Y, Campanella E, Low PS, Goldman ID (2009): A role for the protoncoupled folate transporter (PCFT-SLC46A1) in folate receptor-mediated endocytosis. J Biol Chem $\underline{284}, 4267-4274$ 


\section{$7 \quad$ Anhang}

Tabelle A-1: Kontrollgruppe. Quantitative DTI- und MTsat-Daten

\begin{tabular}{|c|c|c|c|c|c|c|c|}
\hline Kohorte & Anzahl & Alter & ROI & FA & $\mathrm{AD}$ & RD & MD \\
\hline \multicolumn{8}{|c|}{ Kontrolle Protokoll STEAM 1 (STEAM_P1) } \\
\hline $1,6-$ & $\mathrm{n}=1$ & 3,0 & GCC & 0,66 & 1,54 & 0,44 & 0,80 \\
\hline \multirow[t]{3}{*}{$4,9 \mathrm{~J}}$. & $(0 \mathrm{w})$ & & SCC & 0,67 & 1,49 & 0,43 & 0,78 \\
\hline & & & $\mathrm{CC}$ & 0,62 & 1,55 & 0,51 & 0,85 \\
\hline & & & Pyr & 0,45 & 1,26 & 0,59 & 0,82 \\
\hline \multirow{4}{*}{$\begin{array}{l}5,0- \\
15,0 \mathrm{~J} .\end{array}$} & $\mathrm{n}=3$ & $9,0 \pm$ & GCC & $0,64 \pm 0,05$ & $1,47 \pm 0,03$ & $0,46 \pm 0,07$ & $0,79 \pm 0,04$ \\
\hline & $(2 \mathrm{w})$ & 2,3 & SCC & $0,70 \pm 0,01$ & $1,54 \pm 0,06$ & $0,40 \pm 0,01$ & $0,78 \pm 0,03$ \\
\hline & & & $\mathrm{CC}$ & $0,62 \pm 0,04$ & $1,54 \pm 0,06$ & $0,51 \pm 0,05$ & $0,85 \pm 0,05$ \\
\hline & & & Pyr & $0,46 \pm 0,03$ & $1,20 \pm 0,05$ & $0,56 \pm 0,10$ & $0,77 \pm 0,02$ \\
\hline \multicolumn{8}{|c|}{ Kontrolle Protokoll STEAM 2 (STEAM_P2) } \\
\hline $1,6-$ & $\mathrm{n}=2$ & $3,6 \pm$ & GCC & $0,76 \pm 0,00$ & $1,47 \pm 0,10$ & $0,31 \pm 0,04$ & $0,69 \pm 0,06$ \\
\hline \multirow[t]{3}{*}{$4,9 \mathrm{~J}}$. & $(0 \mathrm{w})$ & 1,4 & SCC & $0,76 \pm 0,11$ & $1,37 \pm 0,06$ & $0,31 \pm 0,13$ & $0,66 \pm 0,07$ \\
\hline & & & CC & $0,76 \pm 0,05$ & $1,38 \pm 0,05$ & $0,31 \pm 0,04$ & $0,67 \pm 0,04$ \\
\hline & & & Pyr & $0,60 \pm 0,07$ & $1,19 \pm 0,15$ & $0,40 \pm 0,01$ & $0,68 \pm 0,06$ \\
\hline \multirow{4}{*}{$\begin{array}{l}5,0- \\
15,0 \mathrm{~J}\end{array}$} & $\mathrm{n}=15$ & $9,7 \pm$ & GCC & $0,78 \pm 0,05$ & $1,35 \pm 0,09$ & $0,28 \pm 0,04$ & $0,63 \pm 0,04$ \\
\hline & $(5 \mathrm{w})$ & 3,4 & SCC & $0,78 \pm 0,04$ & $1,37 \pm 0,06$ & $0,28 \pm 0,04$ & $0,64 \pm 0,03$ \\
\hline & & & CC & $0,74 \pm 0,04$ & $1,31 \pm 0,08$ & $0,30 \pm 0,04$ & $0,64 \pm 0,04$ \\
\hline & & & Pyr & $0,59 \pm 0,05$ & $1,07 \pm 0,06$ & $0,39 \pm 0,05$ & $0,61 \pm 0,04$ \\
\hline \multicolumn{8}{|c|}{ Kontrolle Protokoll EPI } \\
\hline $1,6-$ & $\mathrm{n}=1$ & 1,6 & GCC & 0,80 & 1,86 & 0,32 & 0,84 \\
\hline \multirow[t]{3}{*}{ 4,9 J. } & $(0 \mathrm{w})$ & & SCC & 0,77 & 2,08 & 0,45 & 0,99 \\
\hline & & & CC & 0,74 & 1,86 & 0,42 & 0,90 \\
\hline & & & Pyr & 0,64 & 1,38 & 0,42 & 0,74 \\
\hline $5,0-$ & $\mathrm{n}=2$ & $13,9 \pm$ & GCC & $0,85 \pm 0,02$ & $1,71 \pm 0,14$ & $0,23 \pm 0,02$ & $0,73 \pm 0,03$ \\
\hline \multirow[t]{3}{*}{$15,0 \mathrm{~J}}$. & $(1 \mathrm{w})$ & 0,7 & SCC & $0,87 \pm 0,02$ & $1,84 \pm 0,24$ & $0,23 \pm 0,01$ & $0,77 \pm 0,08$ \\
\hline & & & $\mathrm{CC}$ & $0,83 \pm 0,02$ & $1,70 \pm 0,21$ & $0,25 \pm 0,01$ & $0,73 \pm 0,07$ \\
\hline & & & Pyr & $0,73 \pm 0,04$ & $1,42 \pm 0,02$ & $0,33 \pm 0,05$ & $0,70 \pm 0,04$ \\
\hline
\end{tabular}

\section{Kontrolle MT}

$\begin{array}{llllr}1,6- & \mathrm{n}=4 & 2,71 \pm & \text { GCC } & 2,71 \pm 0,38 \\ 4,9 \mathrm{~J} . & (0 \mathrm{w}) & 0,92 & \text { SCC } & 2,74 \pm 0,27 \\ & & & \text { WMLF } & 2,62 \pm 0,33 \\ & & & \text { WMLPO } & 2,52 \pm 0,24 \\ & & & & 3,05 \pm 0,19 \\ 5,0- & \mathrm{n}=27 & 9,2 \pm & \text { GCC } & 2,89 \pm 0,18 \\ 15,0 \mathrm{~J} . & (9 \mathrm{w}) & 2,85 & \text { SCC } & 2,82 \pm 0,16 \\ & & & \text { WMLF } & 2,74 \pm 0,12 \\ & & & \text { WMLPO }\end{array}$

m: männlich. w: weiblich. GCC: Genu. SCC: Splenium. CC: Corpus Callosum. Pyr: Pyramidenbahn. WMLF: Weiße Hirnsubstanz links frontal. WMLPO: Weiße Hirnsubstanz links parieto-okzipital. FA (p. u.). AD, RD, MD (Einheit in $10-3 \mathrm{~mm}^{2} \cdot \mathrm{s}^{-1}$, MTsat (p. u.). Alter (in J.). Aufgeführte SD: Einfache SD. Protokoll STEAM 1: Richtungsprotokoll für STEAM-Sequenz an der UMG bis 06/2008. Protokoll STEAM 2: Richtungsprotokoll für STEAM-Sequenz an der UMG seit 07/2008 
Tabelle A-2: ccALD-Patient 1. Quantitative DTI- und MTsat-Daten

\begin{tabular}{|c|c|c|c|c|c|c|c|}
\hline Messung & Alter & ROI & FA & $\mathrm{AD}$ & RD & MD & MTsat \\
\hline 1 & 2,5 & SCC & $0,84 \pm 0,01$ & $1,38 \pm 0,04$ & $0,21 \pm 0,01$ & $0,60 \pm 0,01$ & $2,57 \pm 0,25$ \\
\hline 2 & 2,8 & SCC & $0,77 \pm 0,03$ & $1,35 \pm 0,01$ & $0,27 \pm 0,03$ & $0,63 \pm 0,02$ & $2,76 \pm 0,26$ \\
\hline 3 & 3,3 & SCC & $0,78 \pm 0,02$ & $1,32 \pm 0,05$ & $0,26 \pm 0,04$ & $0,61 \pm 0,04$ & $2,86 \pm 0,15$ \\
\hline $4^{*}$ & 3,8 & SCC & & & & & $2,82 \pm 0,22$ \\
\hline 5 & 4,4 & SCC & $0,76 \pm 0,01$ & $1,30 \pm 0,03$ & $0,27 \pm 0,01$ & $0,61 \pm 0,02$ & $2,45 \pm 0,28$ \\
\hline 6 & 5,0 & SCC & $0,72 \pm 0,03$ & $1,22 \pm 0,02$ & $0,30 \pm 0,02$ & $0,61 \pm 0,02$ & $2,72 \pm 0,23$ \\
\hline 7 & 5,6 & SCC & $0,73 \pm 0,02$ & $1,27 \pm 0,01$ & $0,30 \pm 0,02$ & $0,63 \pm 0,02$ & $2,64 \pm 0,21$ \\
\hline $8^{* *}$ & 7,3 & SCC & $0,55 \pm 0,01$ & $1,23 \pm 0,01$ & $0,48 \pm 0,01$ & $0,73 \pm 0,01$ & $2,10 \pm 0,60$ \\
\hline 1 & 2,5 & SCC sup. & $0,82 \pm 0,02$ & $1,47 \pm 0,07$ & $0,24 \pm 0,04$ & $0,65 \pm 0,05$ & $2,62 \pm 0,29$ \\
\hline 2 & 2,8 & SCC sup. & $0,83 \pm 0,04$ & $1,34 \pm 0,02$ & $0,20 \pm 0,04$ & $0,58 \pm 0,03$ & $2,76 \pm 0,17$ \\
\hline 3 & 3,3 & SCC sup. & $0,79 \pm 0,02$ & $1,43 \pm 0,13$ & $0,26 \pm 0,05$ & $0,65 \pm 0,08$ & $2,87 \pm 0,14$ \\
\hline $4^{*}$ & 3,8 & SCC sup. & & & & & $2,84 \pm 0,15$ \\
\hline 5 & 4,4 & SCC sup. & $0,79 \pm 0,02$ & $1,32 \pm 0,03$ & $0,25 \pm 0,03$ & $0,61 \pm 0,03$ & $2,22 \pm 0,18$ \\
\hline 6 & 5,0 & SCC sup. & $0,74 \pm 0,02$ & $1,20 \pm 0,05$ & $0,27 \pm 0,02$ & $0,58 \pm 0,03$ & $2,73 \pm 0,32$ \\
\hline 7 & 5,6 & SCC sup. & $0,73 \pm 0,02$ & $1,31 \pm 0,04$ & $0,30 \pm 0,01$ & $0,64 \pm 0,01$ & $2,55 \pm 0,44$ \\
\hline $8^{* *}$ & 7,3 & SCC sup. & $0,49 \pm 0,03$ & $1,18 \pm 0,02$ & $0,51 \pm 0,04$ & $0,73 \pm 0,03$ & $1,22 \pm 0,19$ \\
\hline 1 & 2,5 & SCC inf & $0,88 \pm 0,01$ & $1,32 \pm 0,06$ & $0,16 \pm 0,01$ & $0,55 \pm 0,02$ & $2,80 \pm 0,29$ \\
\hline 2 & 2,8 & SCC inf & $0,73 \pm 0,06$ & $1,35 \pm 0,02$ & $0,31 \pm 0,08$ & $0,66 \pm 0,06$ & $2,83 \pm 0,24$ \\
\hline 3 & 3,3 & SCC inf & $0,76 \pm, 03$ & $1,27 \pm 0,01$ & $0,27 \pm 0,04$ & $0,60 \pm 0,02$ & $2,84 \pm 0,20$ \\
\hline $4^{*}$ & 3,8 & SCC inf & & & & & $2,93 \pm 0,32$ \\
\hline 5 & 4,4 & SCC inf & $0,73 \pm 0,03$ & $1,27 \pm 0,04$ & $0,30 \pm 0,03$ & $0,62 \pm 0,02$ & $2,32 \pm 0,27$ \\
\hline 6 & 5,0 & SCC inf & $0,70 \pm 0,03$ & $1,24 \pm 0,01$ & $0,33 \pm 0,00$ & $0,63 \pm 0,01$ & $2,73 \pm 0,22$ \\
\hline 7 & 5,6 & SCC inf & $0,75 \pm 0,01$ & $1,18 \pm 0,01$ & $0,27 \pm 0,02$ & $0,58 \pm 0,01$ & $2,70 \pm 0,37$ \\
\hline $8^{* *}$ & 7,3 & SCC inf & $0,61 \pm 0,03$ & $1,31 \pm 0,02$ & $0,44 \pm 0,03$ & $0,73 \pm 0,02$ & $2,74 \pm 0,20$ \\
\hline 1 & 2,5 & SCC Läsion & 0,73 & 1,50 & 0.34 & 0,73 & 2,74 \\
\hline 2 & 2,8 & SCC Läsion & 0,82 & 1,41 & 0,21 & 0,61 & 2,71 \\
\hline 3 & 3,3 & SCC Läsion & 0,89 & 1,27 & 0,20 & 0,56 & 2,79 \\
\hline $4^{*}$ & 3,8 & SCC Läsion & & & & & 2,84 \\
\hline 5 & 4,4 & SCC Läsion & 0,78 & 1,34 & 0,22 & 0,61 & $2,02 \pm 0,12$ \\
\hline 6 & 5,0 & SCC Läsion & 0,59 & 1,15 & 0,39 & 0,64 & $2,52 \pm 0,06$ \\
\hline 7 & 5,6 & SCC Läsion & 0,53 & 1,14 & 0,45 & 0,61 & $1,54 \pm 0,19$ \\
\hline $8^{* *}$ & 7,3 & SCC Läsion & 0,36 & 1,01 & 0,57 & 0,62 & $1,05 \pm 0,08$ \\
\hline \multicolumn{8}{|l|}{ Kontrolle } \\
\hline \multirow[t]{4}{*}{ STEAM_P2 } & $1,6-4,9$ & SCC & $0,76 \pm 0,11$ & $1,37 \pm 0,06$ & $0,31 \pm 0,13$ & $0,66 \pm 0,07$ & $2,74 \pm 0,27$ \\
\hline & & $2 \mathrm{SD}$ & $0,54-0,98$ & $1,25-1,49$ & $0,05-0,57$ & $0,52-0,80$ & $2,22-3,28$ \\
\hline & $5,0-15,0$ & SCC & $0,78 \pm 0,04$ & $1,37 \pm 0,06$ & $0,28 \pm 0,04$ & $0,64 \pm 0,03$ & $2,89 \pm 0,18$ \\
\hline & & $2 \mathrm{SD}$ & $0,70-0,86$ & $1,25-1,49$ & $0,20-0,36$ & $0,58-0,70$ & $2,53-3,25$ \\
\hline
\end{tabular}

Fett: Wert außerhalb des 2SD-Bereiches. *Keine STEAM-Diffusionsdaten o. MT-Daten vorhanden. **Z.n. HSZT. SCC: Splenium. SCC sup.: Splenium superior. SCC inf.: Splenium inferior. SCC Läsion: Lokalisation der mit 5,6 Jahren beschriebenen Läsion im Splenium. FA (p. u.). AD, RD, MD (Einheit in 10-3 $\mathrm{mm}^{2} \cdot \mathrm{s}^{-1}$ ), MTsat (p. u.). Alter: (in J.). Alle Werte wenn nicht als 2SD angebenen: Wert \pm SD. Die aufgeführten Diffusionsdaten wurden mit einer STEAM-Sequenz und Richtungsprotokoll 2 (STEAM_P2) aufgenommen. 
Tabelle A-3: ccALD-Patient 2. Quantitative DTI- und MTsat-Daten

\begin{tabular}{|c|c|c|c|c|c|c|c|}
\hline Messung & Alter & ROI & FA & $\mathrm{AD}$ & RD & MD & MTsat \\
\hline 1 & 5,2 & SCC & $0,76 \pm 0,03$ & $1,46 \pm 0,05$ & $0,31 \pm 0,05$ & $0,70 \pm 0,04$ & $2,59 \pm 0,26$ \\
\hline 2 & 5,5 & SCC & $0,81 \pm 0,03$ & $1,45 \pm 0,01$ & $0,25 \pm 0,04$ & $0,65 \pm 0,03$ & $2,79 \pm 0,45$ \\
\hline 3 & 6,0 & SCC & $0,78 \pm 0,03$ & $1,47 \pm 0,04$ & $0,30 \pm 0,04$ & $0,69 \pm 0,03$ & $2,74 \pm 0,30$ \\
\hline 4 & 6,3 & SCC & $0,78 \pm 0,04$ & $1,54 \pm 0,05$ & $0,30 \pm 0,05$ & $0,71 \pm 0,04$ & $2,80 \pm 0,20$ \\
\hline 5 & 6,8 & SCC & $0,77 \pm 0,03$ & $1,44 \pm 0,05$ & $0,29 \pm 0,04$ & $0,67 \pm 0,04$ & $2,68 \pm 0,27$ \\
\hline 1 & 5,2 & SCC sup. & $0,76 \pm 0,02$ & $1,45 \pm 0,04$ & $0,31 \pm 0,02$ & $0,69 \pm 0,02$ & $2,61 \pm 0,19$ \\
\hline 2 & 5,5 & SCC sup. & $0,75 \pm 0,04$ & $1,47 \pm 0,11$ & $0,33 \pm 0,08$ & $0,71 \pm 0,09$ & $2,91 \pm 0,21$ \\
\hline 3 & 6,0 & SCC sup. & $0,80 \pm 0,03$ & $1,44 \pm 0,01$ & $0,26 \pm 0,04$ & $0,66 \pm 0,03$ & $2,76 \pm 0,21$ \\
\hline 4 & 6,3 & SCC sup. & $0,77 \pm 0,03$ & $1,51 \pm 0,08$ & $0,31 \pm 0,05$ & $0,71 \pm 0,05$ & $2,75 \pm 0,20$ \\
\hline 5 & 6,8 & SCC sup. & $0,76 \pm 0,03$ & $1,41 \pm 0,02$ & $0,30 \pm 0,04$ & $0,67 \pm 0,04$ & $2,34 \pm 0,29$ \\
\hline 1 & 5,2 & SCC inf & $0,75 \pm 0,05$ & $1,43 \pm 0,09$ & $0,30 \pm 0,05$ & $0,68 \pm 0,06$ & $2,64 \pm 0,20$ \\
\hline 2 & 5,5 & SCC inf & $0,75 \pm 0,01$ & $1,51 \pm 0,07$ & $0,34 \pm 0,01$ & $0,73 \pm 0,03$ & $3,00 \pm 0,26$ \\
\hline 3 & 6,0 & SCC inf & $0,76 \pm 0,03$ & $1,52 \pm 0,12$ & $0,35 \pm 0,07$ & $0,74 \pm 0,09$ & $2,91 \pm 0,17$ \\
\hline 4 & 6,3 & SCC inf & $0,80 \pm 0,05$ & $1,57 \pm 0,01$ & $0,28 \pm 0,07$ & $0,71 \pm 0,05$ & $2,81 \pm 0,20$ \\
\hline 5 & 6,8 & SCC inf & $0,79 \pm 0,02$ & $1,47 \pm 0,08$ & $0,28 \pm 0,03$ & $0,67 \pm 0,05$ & $2,64 \pm 0,20$ \\
\hline 1 & 5,2 & SCC Läsion & & & & & \\
\hline 2 & 5,5 & SCC Läsion & 0,79 & 1,32 & 0,25 & 0,63 & $2,68 \pm 0,02$ \\
\hline 3 & 6,0 & SCC Läsion & 0,60 & 1,57 & 0,45 & 0,82 & $2,44 \pm 0,08$ \\
\hline 4 & 6,3 & SCC Läsion & 0,67 & 1,62 & 0,45 & 0,83 & $2,25 \pm 0,09$ \\
\hline 5 & 6,8 & SCC Läsion & 0,60 & 1,30 & 0,43 & 0,72 & $1,67 \pm 0,09$ \\
\hline \multicolumn{8}{|l|}{ Kontrolle } \\
\hline \multirow[t]{2}{*}{ STEAM_P2 } & $5,0-15,0$ & SCC & $0,78 \pm 0,04$ & $1,37 \pm 0,06$ & $0,28 \pm 0,04$ & $0,64 \pm 0,03$ & $2,89 \pm 0,18$ \\
\hline & & $2 \mathrm{SD}$ & $0,70-0,86$ & $1,25-1,49$ & $0,20-0,36$ & $0,58-0,70$ & $2,53-3,25$ \\
\hline
\end{tabular}

Fett: Wert außerhalb des 2SD-Bereiches. *Keine STEAM-Diffusionsdaten o. MT-Daten vorhanden. ${ }^{* * Z}$.n. HSZT. SCC: Splenium. SCC sup.: Splenium superior. SCC inf.: Splenium inferior. SCC Läsion: Lokalisation der mit 5,6 Jahren beschriebenen Läsion im Splenium. FA (p. u.). AD, RD, MD (Einheit in 10-3 $\mathrm{mm}^{2} \cdot \mathrm{s}^{-1}$ ), MTsat (p. u.). Alter: (in J.). Alle Werte wenn nicht als 2SD angebenen: Wert \pm SD. Die aufgeführten Diffusionsdaten wurden mit einer STEAM-Sequenz und Richtungsprotokoll 2 (STEAM_P2) aufgenommen.

Tabelle A-4: PMS-Patienten 3-7. Quantitative DTI- und MTsat-Daten

\begin{tabular}{|c|c|c|c|c|c|c|c|}
\hline Patient & Alter & ROI & FA & $\mathrm{AD}$ & RD & MD & MTsat \\
\hline \multirow[t]{6}{*}{3} & 7,9 & GCC & $0,50 \pm 0,01$ & $2,24 \pm 0,09$ & $0,96 \pm 0,02$ & $1,39 \pm 0,04$ & $0,85 \pm 0,23$ \\
\hline & & SCC & $0,43 \pm 0,04$ & $2,23 \pm 0,05$ & $1,09 \pm 0,13$ & $1,47 \pm 0,10$ & $1,08 \pm 0,33$ \\
\hline & & CC & $0,40 \pm 0,04$ & $2,18 \pm 0,05$ & $1,13 \pm 0,13$ & $1,48 \pm 0,10$ & \\
\hline & & Pyr & 0,60 & 1,40 & 0,48 & 0,79 & \\
\hline & & WMLF & & & & & $1,16 \pm 0,10$ \\
\hline & & WMLPO & & & & & $1,07 \pm 0,10$ \\
\hline \multirow[t]{6}{*}{$4 *$} & 1,5 & GCC & & & & & $1,08 \pm 0,40$ \\
\hline & & SCC & & & & & $1,18 \pm 0,23$ \\
\hline & & $\mathrm{CC}$ & & & & & \\
\hline & & Pyr & & & & & \\
\hline & & WMLF & & & & & $1,04 \pm 0,08$ \\
\hline & & WMLPO & & & & & $1,13 \pm 0,16$ \\
\hline
\end{tabular}




\begin{tabular}{|c|c|c|c|c|c|c|c|}
\hline Patient & Alter & ROI & FA & $\mathrm{AD}$ & RD & MD & MTsat \\
\hline \multirow[t]{6}{*}{4} & 6,9 & GCC & $0,70 \pm 0,05$ & $2,22 \pm 0,02$ & $0,59 \pm 0,08$ & $1,13 \pm 0,05$ & $1,72 \pm 0,27$ \\
\hline & & SCC & $0,79 \pm 0,02$ & $1,96 \pm 0,07$ & $0,36 \pm 0,03$ & $0,90 \pm 0,01$ & $1,75 \pm 0,37$ \\
\hline & & CC & $0,67 \pm 0,02$ & $2,12 \pm 0,07$ & $0,62 \pm 0,03$ & $1,12 \pm 0,02$ & \\
\hline & & Pyr & 0,66 & 1,47 & 0,42 & 0,77 & \\
\hline & & WMLF & & & & & $1,02 \pm 0,13$ \\
\hline & & WMLPO & & & & & $1,09 \pm 0,15$ \\
\hline \multirow[t]{6}{*}{5} & 3,2 & GCC & $0,60 \pm 0,01$ & $1,81 \pm 0,06$ & $0,61 \pm 0,03$ & $1,01 \pm 0,02$ & $0,77 \pm 0,18$ \\
\hline & & SCC & $0,66 \pm 0,02$ & $2,06 \pm 0,08$ & $0,59 \pm 0,05$ & $1,08 \pm 0,06$ & $0,66 \pm 0,22$ \\
\hline & & $\mathrm{CC}$ & $0,60 \pm 0,02$ & $1,83 \pm 0,08$ & $0,61 \pm 0,05$ & $1,01 \pm 0,06$ & \\
\hline & & Pyr & 0,58 & 1,54 & 0,55 & 0,88 & \\
\hline & & WMLF & & & & & $0,90 \pm 0,16$ \\
\hline & & WMLPO & & & & & $0,76 \pm 0,08$ \\
\hline \multirow[t]{6}{*}{5} & 4,4 & GCC & $0,53 \pm 0,06$ & $1,83 \pm 0,12$ & $0,53 \pm 0,02$ & $0,96 \pm 0,33$ & $0,54 \pm 0,05$ \\
\hline & & SCC & $0,53 \pm 0,05$ & $2,32 \pm 0,22$ & $0,67 \pm 0,14$ & $1,37 \pm 0,09$ & $0,49 \pm 0,06$ \\
\hline & & $\mathrm{CC}$ & $0,63 \pm 0,05$ & $2,14 \pm 0,11$ & $0,67 \pm 0,14$ & $1,26 \pm 0,09$ & \\
\hline & & Pyr & 0,66 & 1,58 & 0,45 & 0,84 & \\
\hline & & WMLF & & & & & $0,88 \pm 0,04$ \\
\hline & & WMLPO & & & & & $0,86 \pm 0,04$ \\
\hline \multirow[t]{6}{*}{6} & 2,4 & GCC & $0,73 \pm 0,04$ & $2,18 \pm 0,12$ & $0,51 \pm 0,04$ & $1,07 \pm 0,01$ & $0,65 \pm 0,29$ \\
\hline & & SCC & $0,75 \pm 0,04$ & $2,32 \pm 0,11$ & $0,5 \pm 0,04$ & $1,1 \pm 0,01$ & $0,58 \pm 0,28$ \\
\hline & & $\mathrm{CC}$ & $0,67 \pm 0,04$ & $2,14 \pm 0,11$ & $0,6 \pm 0,04$ & $1,11 \pm 0,01$ & \\
\hline & & Pyr & $0,58 \pm 0,00$ & $1,58 \pm 0,04$ & $0,56 \pm 0,00$ & $0,90 \pm 0,00$ & \\
\hline & & WMLF & & & & & $0,77 \pm 0,09$ \\
\hline & & WMLPO & & & & & $1,01 \pm 0,10$ \\
\hline \multirow[t]{6}{*}{6} & 2,8 & GCC & $0,73 \pm 0,05$ & $2,14 \pm 0,09$ & $0,49 \pm 0,07$ & $1,04 \pm 0,04$ & $0,70 \pm 0,68$ \\
\hline & & SCC & $0,77 \pm 0,01$ & $2,37 \pm 0,04$ & $0,48 \pm 0,02$ & $1,11 \pm 0,00$ & $0,53 \pm 0,30$ \\
\hline & & $\mathrm{CC}$ & $0,68 \pm 0,01$ & $2,14 \pm 0,04$ & $0,59 \pm 0,02$ & $1,11 \pm 0,00$ & \\
\hline & & Pyr & 0,60 & 1,56 & 0,51 & 0,86 & \\
\hline & & WMLF & & & & & $0,79 \pm 0,11$ \\
\hline & & WMLPO & & & & & $1,02 \pm 0,09$ \\
\hline \multirow[t]{6}{*}{6} & 3,6 & GCC & $0,69 \pm 0,05$ & $2,37 \pm 0,24$ & $0,63 \pm 0,07$ & $1,21 \pm 0,09$ & $0,69 \pm 0,28$ \\
\hline & & SCC & $0,72 \pm 0,03$ & $2,43 \pm 0,08$ & $0,59 \pm 0,07$ & $1,21 \pm 0,07$ & $0,68 \pm 0,28$ \\
\hline & & $\mathrm{CC}$ & $0,66 \pm 0,03$ & $2,26 \pm 0,08$ & $0,65 \pm 0,07$ & $1,19 \pm 0,07$ & \\
\hline & & Pyr & 0,59 & 1,58 & 0,54 & 0,89 & \\
\hline & & WMLF & & & & & $0,89 \pm 0,12$ \\
\hline & & WMLPO & & & & & $1,04 \pm 0,10$ \\
\hline \multirow[t]{6}{*}{$6^{*}$} & 4,7 & GCC & $0,63 \pm 0,06$ & $2,26 \pm 0,36$ & $0,72 \pm 0,06$ & $1,23 \pm 0,01$ & \\
\hline & & SCC & $0,61 \pm 0,04$ & $2,53 \pm 0,15$ & $0,85 \pm 0,02$ & $1,39 \pm 0,11$ & \\
\hline & & $\mathrm{CC}$ & $0,58 \pm 0,04$ & $2,60 \pm 0,15$ & $0,80 \pm 0,02$ & $1,25 \pm 0,11$ & \\
\hline & & Pyr & 0,61 & 1,56 & 0,49 & 0,83 & \\
\hline & & WMLF & & & & & \\
\hline & & WMLPO & & & & & \\
\hline \multirow[t]{4}{*}{7} & 11,2 & GCC & $0,50 \pm 0,03$ & $2,60 \pm 0,36$ & $1,16 \pm 0,17$ & $1,64 \pm 0,23$ & $0,68 \pm 0,55$ \\
\hline & & SCC & $0,58 \pm 0,07$ & $2,53 \pm 0,15$ & $0,91 \pm 0,12$ & $1,45 \pm 0,09$ & $0,36 \pm 0,16$ \\
\hline & & CC & $0,42 \pm 0,07$ & $2,60 \pm 0,15$ & $1,32 \pm 0,12$ & $1,74 \pm 0,09$ & \\
\hline & & Pyr & 0,55 & 1,56 & 0,51 & 0,86 & \\
\hline
\end{tabular}




\begin{tabular}{|c|c|c|c|c|c|c|c|}
\hline Patient & Alter & ROI & FA & $\mathrm{AD}$ & RD & MD & MTsat \\
\hline & & WMLF & & & & & $0,74 \pm 0,17$ \\
\hline & & WMLPO & & & & & $0,60 \pm 0,16$ \\
\hline \multicolumn{8}{|c|}{ Kontrolle } \\
\hline \multirow[t]{4}{*}{ EPI } & $1,6-$ & GCC & 0,80 & 1,86 & 0,32 & 0,84 & \\
\hline & $4,9 \mathrm{~J}$. & SCC & 0,77 & 2,08 & 0,45 & 0,99 & \\
\hline & & $\mathrm{CC}$ & 0,74 & 1,86 & 0,42 & 0,90 & \\
\hline & & Pyr & 0,64 & 1,38 & 0,42 & 0,74 & \\
\hline \multirow[t]{4}{*}{ EPI } & $5,0-$ & GCC & $0,85 \pm 0,02$ & $1,71 \pm 0,14$ & $0,23 \pm 0,02$ & $0,73 \pm 0,03$ & \\
\hline & $15,0 \mathrm{~J}$. & SCC & $0,87 \pm 0,02$ & $1,84 \pm 0,24$ & $0,23 \pm 0,01$ & $0,77 \pm 0,08$ & \\
\hline & & $\mathrm{CC}$ & $0,83 \pm 0,02$ & $1,70 \pm 0,21$ & $0,25 \pm 0,01$ & $0,73 \pm 0,07$ & \\
\hline & & Pyr & $0,73 \pm 0,04$ & $1,42 \pm 0,02$ & $0,33 \pm 0,05$ & $0,70 \pm 0,04$ & \\
\hline \multirow[t]{4}{*}{ MT } & $1,6-$ & GCC & & & & & $2,71 \pm 0,38$ \\
\hline & $4,9 \mathrm{~J}$. & SCC & & & & & $2,74 \pm 0,27$ \\
\hline & & WMLF & & & & & $2,62 \pm 0,33$ \\
\hline & & WMLPO & & & & & $2,52 \pm 0,24$ \\
\hline \multirow[t]{4}{*}{ MT } & $5,0-$ & GCC & & & & & $3,05 \pm 0,19$ \\
\hline & $15,0 \mathrm{~J}$. & SCC & & & & & $2,89 \pm 0,18$ \\
\hline & & WMLF & & & & & $2,82 \pm 0,16$ \\
\hline & & WMLPO & & & & & $2,74 \pm 0,12$ \\
\hline
\end{tabular}

Fett: Wert außerhalb des 2SD-Bereiches. *Keine STEAM-Diffusionsdaten o. MT-Daten vorhanden GCC: Genu. SCC: Splenium. CC: Corpus Callosum. Pyr: Pyramidenbahn. WMLF: Weiße Hirnsubstanz links frontal. WMLPO: Weiße Hirnsubstanz links parieto-okzipital. . FA (p. u.). AD, RD, MD (Einheit in 10-3 $\mathrm{mm}^{2} \cdot \mathrm{s}^{-1}$ ), MTsat (p. u.). Alter: (in J.). Alle Werte wenn nicht als 2SD angebenen: Wert \pm SD. Die aufgeführten Diffusionsdaten wurden mit einer EPI-Sequenz aufgenommen.

Tabelle A-5: cFD-Patient 8. Quantitative DTI- und MTsat-Daten

\begin{tabular}{|c|c|c|c|c|c|c|c|}
\hline Messung & Alter & ROI & FA & $\mathrm{AD}$ & RD & MD & MTsat \\
\hline \multirow[t]{5}{*}{$1^{* *}$} & 3,8 & GCC & $0,63 \pm 0,03$ & $1,72 \pm 0,05$ & $0,55 \pm 0,05$ & $0,94 \pm 0,03$ & $1,88 \pm 0,22$ \\
\hline & & SCC & $0,58 \pm 0,03$ & $1,80 \pm 0,05$ & $0,66 \pm 0,04$ & $1,04 \pm 0,03$ & $2,33 \pm 0,22$ \\
\hline & & $\mathrm{CC}$ & $0,57 \pm 0,03$ & $1,77 \pm 0,05$ & $0,66 \pm 0,04$ & $1,03 \pm 0,03$ & \\
\hline & & WMLF & & & & & $1,36 \pm 0,12$ \\
\hline & & WMLPO & & & & & $1,06 \pm 0,09$ \\
\hline \multirow[t]{5}{*}{$2 * *$} & 4,3 & GCC & $0,60 \pm 0,01$ & $1,74 \pm 0,08$ & $0,59 \pm 0,02$ & $0,97 \pm 0,04$ & $2,14 \pm 0,58$ \\
\hline & & SCC & $0,62 \pm 0,03$ & $1,77 \pm 0,04$ & $0,58 \pm 0,06$ & $0,98 \pm 0,05$ & $2,71 \pm 0,81$ \\
\hline & & CC & $0,54 \pm 0,03$ & $1,74 \pm 0,04$ & $0,70 \pm 0,06$ & $1,04 \pm 0,05$ & \\
\hline & & WMLF & & & & & $1,75 \pm 0,16$ \\
\hline & & WMLPO & & & & & $1,56 \pm 0,16$ \\
\hline \multirow[t]{5}{*}{$3^{*}$} & 4,9 & GCC & & & & & $2,26 \pm 0,67$ \\
\hline & & SCC & & & & & $2,73 \pm 0,53$ \\
\hline & & $\mathrm{CC}$ & & & & & \\
\hline & & WMLF & & & & & $1,66 \pm 0,13$ \\
\hline & & WMLPO & & & & & $1,32 \pm 0,13$ \\
\hline \multirow[t]{2}{*}{$4 * *$} & 5,2 & GCC & $0,64 \pm 0,01$ & $1,68 \pm 0,02$ & $0,52 \pm 0,02$ & $0,90 \pm 0,02$ & $2,47 \pm 0,50$ \\
\hline & & SCC & $0,69 \pm 0,03$ & $1,63 \pm 0,00$ & $0,44 \pm 0,04$ & $0,83 \pm 0,02$ & $2,63 \pm 0,73$ \\
\hline
\end{tabular}




\begin{tabular}{|c|c|c|c|c|c|c|c|}
\hline Messung & Alter & ROI & FA & $\mathrm{AD}$ & RD & MD & MTsat \\
\hline & & $\mathrm{CC}$ & $0,63 \pm 0,03$ & $1,57 \pm 0,00$ & $0,50 \pm 0,04$ & $0,86 \pm 0,02$ & \\
\hline & & WMLF & & & & & $1,79 \pm 0,13$ \\
\hline & & WMLPO & & & & & $1,24 \pm 0,10$ \\
\hline \multirow[t]{5}{*}{5} & 5,5 & GCC & $0,82 \pm 0,03$ & $1,27 \pm 0,11$ & $0,22 \pm 0,07$ & $0,57 \pm 0,08$ & $2,44 \pm 0,58$ \\
\hline & & SCC & $0,89 \pm 0,04$ & $1,37 \pm 0,00$ & $0,15 \pm 0,05$ & $0,55 \pm 0,03$ & $2,47 \pm 0,58$ \\
\hline & & $\mathrm{CC}$ & $0,78 \pm 0,04$ & $1,37 \pm 0,00$ & $0,24 \pm 0,05$ & $0,61 \pm 0,03$ & \\
\hline & & WMLF & & & & & $1,73 \pm 0,20$ \\
\hline & & WMLPO & & & & & $1,27 \pm 0,13$ \\
\hline \multirow[t]{5}{*}{6} & 6,0 & GCC & $0,87 \pm 0,04$ & $1,32 \pm 0,10$ & $0,15 \pm 0,06$ & $0,54 \pm 0,07$ & $2,73 \pm 0,33$ \\
\hline & & SCC & $0,87 \pm 0,03$ & $1,39 \pm 0,05$ & $0,17 \pm 0,04$ & $0,57 \pm 0,04$ & $2,89 \pm 0,62$ \\
\hline & & $\mathrm{CC}$ & $0,77 \pm 0,03$ & $1,35 \pm 0,05$ & $0,34 \pm 0,04$ & $0,68 \pm 0,04$ & \\
\hline & & WMLF & & & & & $1,97 \pm 0,18$ \\
\hline & & WMLPO & & & & & $1,24 \pm 0,12$ \\
\hline \multirow[t]{5}{*}{7} & 6,5 & GCC & $0,83 \pm 0,02$ & $1,39 \pm 0,03$ & $0,21 \pm 0,03$ & $0,60 \pm 0,03$ & $2,82 \pm 0,42$ \\
\hline & & SCC & $0,81 \pm 0,01$ & $1,41 \pm 0,04$ & $0,25 \pm 0,00$ & $0,63 \pm 0,01$ & $2,81 \pm 0,67$ \\
\hline & & CC & $0,70 \pm 0,01$ & $1,36 \pm 0,04$ & $0,37 \pm 0,00$ & $0,70 \pm 0,01$ & \\
\hline & & WMLF & & & & & $2,05 \pm 0,21$ \\
\hline & & WMLPO & & & & & $1,37 \pm 0,14$ \\
\hline \multirow[t]{5}{*}{8} & 7,0 & GCC & $0,86 \pm 0,01$ & $1,37 \pm 0,02$ & $0,17 \pm 0,01$ & $0,57 \pm 0,00$ & $2,84 \pm 0,28$ \\
\hline & & SCC & $0,91 \pm 0,00$ & $1,31 \pm 0,02$ & $0,11 \pm 0,01$ & $0,51 \pm 0,01$ & $2,96 \pm 0,39$ \\
\hline & & CC & $0,83 \pm 0,00$ & $1,37 \pm 0,02$ & $0,2 \pm 0,01$ & $0,59 \pm 0,01$ & \\
\hline & & WMLF & & & & & $2,09 \pm 0,18$ \\
\hline & & WMLPO & & & & & $1,27 \pm 0,16$ \\
\hline \multirow[t]{5}{*}{$9 *$} & 7,5 & GCC & & & & & $2,83 \pm 0,26$ \\
\hline & & SCC & & & & & $2,83 \pm 0,2$ \\
\hline & & CC & & & & & \\
\hline & & WMLF & & & & & $2,22 \pm 0,15$ \\
\hline & & WMLPO & & & & & $1,35 \pm 0,14$ \\
\hline \multirow[t]{5}{*}{10} & 8,0 & GCC & $0,96 \pm 0,00$ & $2,13 \pm 0,11$ & $0,07 \pm 0,01$ & $0,76 \pm 0,03$ & $2,76 \pm 0,27$ \\
\hline & & SCC & $0,87 \pm 0,02$ & $1,87 \pm 0,04$ & $0,22 \pm 0,03$ & $0,77 \pm 0,02$ & $2,51 \pm 0,30$ \\
\hline & & CC & $0,83 \pm 0,02$ & $2,03 \pm 0,04$ & $0,30 \pm 0,03$ & $0,88 \pm 0,02$ & \\
\hline & & WMLF & & & & & $2,26 \pm 0,15$ \\
\hline & & WMLPO & & & & & $1,27 \pm 0,13$ \\
\hline \multirow[t]{5}{*}{11} & 10,2 & GCC & $0,83 \pm 0,03$ & $1,44 \pm 0,06$ & $0,21 \pm 0,03$ & $0,62 \pm 0,04$ & $3,15 \pm 0,19$ \\
\hline & & SCC & $0,84 \pm 0,06$ & $1,34 \pm 0,08$ & $0,20 \pm 0,07$ & $0,58 \pm 0,03$ & $2,92 \pm 0,22$ \\
\hline & & $\mathrm{CC}$ & $0,76 \pm 0,06$ & $1,31 \pm 0,08$ & $0,27 \pm 0,07$ & $0,62 \pm 0,03$ & \\
\hline & & WMLF & & & & & $2,53 \pm 0,14$ \\
\hline & & WMLPO & & & & & $1,10 \pm 0,09$ \\
\hline \multirow[t]{5}{*}{$12 *$} & 12,0 & GCC & & & & & $2,97 \pm 0,22$ \\
\hline & & SCC & & & & & $2,99 \pm 0,23$ \\
\hline & & $\mathrm{CC}$ & & & & & \\
\hline & & WMLF & & & & & $2,55 \pm 0,19$ \\
\hline & & WMLPO & & & & & $1,29 \pm 0,06$ \\
\hline
\end{tabular}




\begin{tabular}{|c|c|c|c|c|c|c|c|}
\hline Messung & Alter & ROI & FA & $\mathrm{AD}$ & RD & MD & MTsat \\
\hline \multicolumn{8}{|l|}{ Kontrolle } \\
\hline \multirow[t]{3}{*}{ STEAM_P1 } & $1,6-$ & GCC & 0,66 & 1,54 & 0,44 & 0,80 & \\
\hline & $4,9 \mathrm{~J}$. & SCC & 0,67 & 1,49 & 0,43 & 0,78 & \\
\hline & & CC & 0,62 & 1,55 & 0,51 & 0,85 & \\
\hline \multirow[t]{3}{*}{ STEAM_P1 } & $5,0-$ & GCC & $0,64 \pm 0,05$ & $1,47 \pm 0,03$ & $0,46 \pm 0,07$ & $0,79 \pm 0,04$ & \\
\hline & $15,0 \mathrm{~J}$. & SCC & $0,70 \pm 0,01$ & $1,54 \pm 0,06$ & $0,40 \pm 0,01$ & $0,78 \pm 0,03$ & \\
\hline & & $\mathrm{CC}$ & $0,62 \pm 0,04$ & $1,54 \pm 0,06$ & $0,51 \pm 0,05$ & $0,85 \pm 0,05$ & \\
\hline \multirow[t]{3}{*}{ STEAM_P2 } & $1,6-$ & GCC & $0,76 \pm 0,00$ & $1,47 \pm 0,10$ & $0,31 \pm 0,04$ & $0,69 \pm 0,06$ & \\
\hline & $4,9 \mathrm{~J}$. & SCC & $0,76 \pm 0,11$ & $1,37 \pm 0,06$ & $0,31 \pm 0,13$ & $0,66 \pm 0,07$ & \\
\hline & & $\mathrm{CC}$ & $0,76 \pm 0,05$ & $1,38 \pm 0,05$ & $0,31 \pm 0,04$ & $0,67 \pm 0,04$ & \\
\hline \multirow[t]{3}{*}{ STEAM_P2 } & $5,0-$ & GCC & $0,78 \pm 0,05$ & $1,35 \pm 0,09$ & $0,28 \pm 0,04$ & $0,63 \pm 0,04$ & \\
\hline & $15,0 \mathrm{~J}$. & SCC & $0,78 \pm 0,04$ & $1,37 \pm 0,04$ & $0,28 \pm 0,04$ & $0,64 \pm 0,03$ & \\
\hline & & $\mathrm{CC}$ & $0,74 \pm 0,04$ & $1,31 \pm 0,08$ & $0,30 \pm 0,04$ & $0,64 \pm 0,04$ & \\
\hline \multirow[t]{4}{*}{ MT } & $1,6-$ & GCC & & & & & $2,71 \pm 0,38$ \\
\hline & $4,9 \mathrm{~J}$. & SCC & & & & & $2,74 \pm 0,27$ \\
\hline & & WMLF & & & & & $2,62 \pm 0,33$ \\
\hline & & WMLPO & & & & & $2,52 \pm 0,24$ \\
\hline \multirow[t]{4}{*}{ MT } & $5,0-$ & GCC & & & & & $3,05 \pm 0,19$ \\
\hline & $15,0 \mathrm{~J}$. & SCC & & & & & $2,89 \pm 0,18$ \\
\hline & & WMLF & & & & & $2,82 \pm 0,16$ \\
\hline & & WMLPO & & & & & $2,74 \pm 0,12$ \\
\hline
\end{tabular}

Fett: Wert außerhalb des 2SD-Bereiches. *Keine STEAM-Diffusionsdaten o. MT-Daten vorhanden **Daten wurde mit STEAM_P1 Protokoll aufgenommen. Nummerierung: Patientennummer. GCC: Genu. SCC: Splenium. CC: Corpus Callosum. WMLF: Weiße Hirnsubstanz links frontal. WMLPO: Weiße Hirnsubstanz links parieto-okzipital. FA (p. u.). AD, RD, MD (Einheit in 10-3 $\mathrm{mm}^{2} \cdot \mathrm{s}^{-1}$ ), MTsat (p. u.). Alter: (in J.). Alle Werte wenn nicht als 2SD angebenen: Wert \pm SD. Die aufgeführten Diffusionsdaten wurden, wenn nicht anders angegeben, mit einer STEAM-Sequenz (Protokoll STEAM_P2) aufgenommen.

Tabelle A-6: cFD-Patientin 9. Quantitative DTI- und MTsat-Daten

\begin{tabular}{|c|c|c|c|c|c|c|c|}
\hline Messung & Alter & ROI & FA & $\mathrm{AD}$ & RD & MD & MTsat \\
\hline \multirow[t]{5}{*}{$1 * *$} & 2,3 & GCC & $0,66 \pm 0,00$ & $1,64 \pm 0,04$ & $0,48 \pm 0,02$ & $0,87 \pm 0,02$ & $2,12 \pm 0,19$ \\
\hline & & SCC & $0,75 \pm 0,01$ & $1,68 \pm 0,04$ & $0,37 \pm 0,01$ & $0,81 \pm 0,01$ & $2,25 \pm 0,37$ \\
\hline & & $\mathrm{CC}$ & $0,53 \pm 0,01$ & $1,67 \pm 0,04$ & $0,60 \pm 0,01$ & $0,96 \pm 0,01$ & \\
\hline & & WMLF & & & & & $1,76 \pm 0,12$ \\
\hline & & WMLPO & & & & & $1,76 \pm 0,16$ \\
\hline \multirow[t]{5}{*}{$2^{* *}$} & 2,6 & GCC & $0,63 \pm 0,02$ & $1,53 \pm 0,04$ & $0,48 \pm 0,04$ & $0,83 \pm 0,04$ & $2,28 \pm 0,22$ \\
\hline & & SCC & $0,68 \pm 0,00$ & $1,74 \pm 0,03$ & $0,49 \pm 0,00$ & $0,90 \pm 0,01$ & $2,29 \pm 0,36$ \\
\hline & & $\mathrm{CC}$ & $0,58 \pm 0,00$ & $1,67 \pm 0,03$ & $0,61 \pm 0,00$ & $0,97 \pm 0,01$ & \\
\hline & & WMLF & & & & & $1,86 \pm 0,13$ \\
\hline & & WMLPO & & & & & $1,80 \pm 0,14$ \\
\hline \multirow[t]{3}{*}{3} & 3,2 & GCC & $0,84 \pm 0,02$ & $1,32 \pm 0,01$ & $0,19 \pm 0,02$ & $0,57 \pm 0,02$ & $2,57 \pm 0,18$ \\
\hline & & SCC & $0,87 \pm 0,01$ & $1,32 \pm 0,02$ & $0,16 \pm 0,01$ & $0,54 \pm 0,01$ & $2,22 \pm 0,32$ \\
\hline & & $\mathrm{CC}$ & $0,79 \pm 0,01$ & $1,36 \pm 0,02$ & $0,26 \pm 0,01$ & $0,63 \pm 0,01$ & \\
\hline
\end{tabular}




\begin{tabular}{|c|c|c|c|c|c|c|c|}
\hline Messung & Alter & ROI & FA & $\mathrm{AD}$ & RD & MD & MTsat \\
\hline & & WMLF & & & & & $2,01 \pm 0,02$ \\
\hline & & WMLPO & & & & & $1,79 \pm 0,19$ \\
\hline \multirow[t]{5}{*}{4} & 3,7 & GCC & $0,86 \pm 0,05$ & $1,37 \pm 0,02$ & $0,17 \pm 0,06$ & $0,57 \pm 0,05$ & $2,75 \pm 0,16$ \\
\hline & & SCC & $0,85 \pm 0,00$ & $1,32 \pm 0,03$ & $0,18 \pm 0,00$ & $0,56 \pm 0,01$ & $2,56 \pm 0,37$ \\
\hline & & $\mathrm{CC}$ & $0,76 \pm 0,00$ & $1,32 \pm 0,03$ & $0,29 \pm 0,00$ & $0,63 \pm 0,01$ & \\
\hline & & WMLF & & & & & $2,24 \pm 0,15$ \\
\hline & & WMLPO & & & & & $2,09 \pm 0,19$ \\
\hline \multirow[t]{5}{*}{5} & 4,3 & GCC & $0,84 \pm 0,03$ & $1,29 \pm 0,05$ & $0,18 \pm 0,04$ & $0,55 \pm 0,04$ & $3,10 \pm 0,18$ \\
\hline & & SCC & $0,86 \pm 0,03$ & $1,35 \pm 0,06$ & $0,18 \pm 0,04$ & $0,57 \pm 0,05$ & $2,70 \pm 0,24$ \\
\hline & & $\mathrm{CC}$ & $0,80 \pm 0,03$ & $1,30 \pm 0,06$ & $0,24 \pm 0,04$ & $0,59 \pm 0,05$ & \\
\hline & & WMLF & & & & & $2,38 \pm 0,16$ \\
\hline & & WMLPO & & & & & $2,29 \pm 0,21$ \\
\hline \multirow[t]{5}{*}{$6^{*}$} & 5,3 & GCC & & & & & $2,99 \pm 0,46$ \\
\hline & & SCC & & & & & $2,76 \pm 0,33$ \\
\hline & & $\mathrm{CC}$ & & & & & \\
\hline & & WMLF & & & & & $2,28 \pm 0,19$ \\
\hline & & WMLPO & & & & & $2,59 \pm 0,27$ \\
\hline \multirow[t]{5}{*}{7} & 6,3 & GCC & $0,79 \pm 0,03$ & $1,36 \pm 0,01$ & $0,25 \pm 0,03$ & $0,62 \pm 0,02$ & $2,79 \pm 0,15$ \\
\hline & & SCC & $0,82 \pm 0,02$ & $1,35 \pm 0,05$ & $0,22 \pm 0,03$ & $0,60 \pm 0,04$ & $2,68 \pm 0,24$ \\
\hline & & $\mathrm{CC}$ & $0,76 \pm 0,02$ & $1,29 \pm 0,05$ & $0,28 \pm 0,03$ & $0,61 \pm 0,04$ & \\
\hline & & WMLF & & & & & $2,66 \pm 0,18$ \\
\hline & & WMLPO & & & & & $2,39 \pm 0,18$ \\
\hline \multirow[t]{5}{*}{8} & 8,3 & GCC & $0,84 \pm 0,04$ & $1,28 \pm 0,01$ & $0,19 \pm 0,03$ & $0,55 \pm 0,03$ & $3,04 \pm 0,13$ \\
\hline & & SCC & $0,81 \pm 0,02$ & $1,35 \pm 0,05$ & $0,23 \pm 0,03$ & $0,60 \pm 0,03$ & $2,72 \pm 0,16$ \\
\hline & & CC & $0,80 \pm 0,02$ & $1,25 \pm 0,05$ & $0,22 \pm 0,03$ & $0,57 \pm 0,03$ & \\
\hline & & WMLF & & & & & $2,66 \pm 0,12$ \\
\hline & & WMLPO & & & & & $2,64 \pm 0,16$ \\
\hline \multirow[t]{5}{*}{9} & 9,4 & GCC & $0,88 \pm 0,02$ & $1,24 \pm 0,00$ & $0,14 \pm 0,02$ & $0,51 \pm 0,01$ & $3,08 \pm 0,15$ \\
\hline & & SCC & $0,81 \pm 0,02$ & $1,24 \pm 0,03$ & $0,22 \pm 0,01$ & $0,56 \pm 0,01$ & $2,72 \pm 0,32$ \\
\hline & & CC & $0,79 \pm 0,02$ & $1,22 \pm 0,03$ & $0,23 \pm 0,01$ & $0,56 \pm 0,01$ & \\
\hline & & WMLF & & & & & $2,69 \pm 0,14$ \\
\hline & & WMLPO & & & & & $2,55 \pm 0,18$ \\
\hline \multirow[t]{5}{*}{10} & 9,9 & GCC & $0,84 \pm 0,04$ & $1,28 \pm 0,06$ & $0,19 \pm 0,04$ & $0,55 \pm 0,01$ & $2,96 \pm 0,18$ \\
\hline & & SCC & $0,77 \pm 0,03$ & $1,31 \pm 0,02$ & $0,27 \pm 0,03$ & $0,62 \pm 0,02$ & $2,72 \pm 0,20$ \\
\hline & & $\mathrm{CC}$ & $0,75 \pm 0,03$ & $1,23 \pm 0,02$ & $0,27 \pm 0,03$ & $0,59 \pm 0,02$ & \\
\hline & & WMLF & & & & & $2,71 \pm 0,13$ \\
\hline & & WMLPO & & & & & $2,72 \pm 0,19$ \\
\hline \multirow[t]{5}{*}{11} & 11,1 & GCC & $0,87 \pm 0,02$ & $1,30 \pm 0,01$ & $0,15 \pm 0,02$ & $0,53 \pm 0,01$ & $3,09 \pm 0,21$ \\
\hline & & SCC & $0,73 \pm 0,03$ & $1,27 \pm 0,08$ & $0,30 \pm 0,03$ & $0,63 \pm 0,04$ & $2,81 \pm 0,23$ \\
\hline & & CC & $0,76 \pm 0,03$ & $1,26 \pm 0,08$ & $0,27 \pm 0,03$ & $0,60 \pm 0,04$ & \\
\hline & & WMLF & & & & & $2,83 \pm 0,14$ \\
\hline & & WMLPO & & & & & $2,86 \pm 0,21$ \\
\hline
\end{tabular}

\section{Kontrolle}




\begin{tabular}{|c|c|c|c|c|c|c|c|}
\hline Messung & Alter & ROI & FA & $\mathrm{AD}$ & RD & MD & MTsat \\
\hline & $4,9 \mathrm{~J}$. & SCC & 0,67 & 1,49 & 0,43 & 0,78 & \\
\hline & & $\mathrm{CC}$ & 0,62 & 1,55 & 0,51 & 0,85 & \\
\hline \multirow[t]{3}{*}{ STEAM_P1 } & $5,0-$ & GCC & $0,64 \pm 0,05$ & $1,47 \pm 0,03$ & $0,46 \pm 0,07$ & $0,79 \pm 0,04$ & \\
\hline & $15,0 \mathrm{~J}$. & SCC & $0,70 \pm 0,01$ & $1,54 \pm 0,06$ & $0,40 \pm 0,01$ & $0,78 \pm 0,03$ & \\
\hline & & $\mathrm{CC}$ & $0,62 \pm 0,04$ & $1,54 \pm 0,06$ & $0,51 \pm 0,05$ & $0,85 \pm 0,05$ & \\
\hline \multirow[t]{3}{*}{ STEAM_P2 } & $1,6-$ & GCC & $0,76 \pm 0,00$ & $1,47 \pm 0,10$ & $0,31 \pm 0,04$ & $0,69 \pm 0,06$ & \\
\hline & $4,9 \mathrm{~J}$. & SCC & $0,76 \pm 0,11$ & $1,37 \pm 0,06$ & $0,31 \pm 0,13$ & $0,66 \pm 0,07$ & \\
\hline & & $\mathrm{CC}$ & $0,76 \pm 0,05$ & $1,38 \pm 0,05$ & $0,31 \pm 0,04$ & $0,67 \pm 0,04$ & \\
\hline \multirow[t]{3}{*}{ STEAM_P2 } & $5,0-$ & GCC & $0,78 \pm 0,05$ & $1,35 \pm 0,09$ & $0,28 \pm 0,04$ & $0,63 \pm 0,04$ & \\
\hline & 15,0 & SCC & $0,78 \pm 0,04$ & $1,37 \pm 0,04$ & $0,28 \pm 0,04$ & $0,64 \pm 0,03$ & \\
\hline & & $\mathrm{CC}$ & $0,74 \pm 0,04$ & $1,31 \pm 0,08$ & $0,30 \pm 0,04$ & $0,64 \pm 0,04$ & \\
\hline \multirow[t]{4}{*}{ MT } & $1,6-$ & GCC & & & & & $2,71 \pm 0,38$ \\
\hline & 4,9 J. & SCC & & & & & $2,74 \pm 0,27$ \\
\hline & & WMLF & & & & & $2,62 \pm 0,33$ \\
\hline & & WMLPO & & & & & $2,52 \pm 0,24$ \\
\hline \multirow[t]{4}{*}{ MT } & $5,0-$ & GCC & & & & & $3,05 \pm 0,19$ \\
\hline & $15,0 \mathrm{~J}$. & SCC & & & & & $2,89 \pm 0,18$ \\
\hline & & WMLF & & & & & $2,82 \pm 0,16$ \\
\hline & & WMLPO & & & & & $2,74 \pm 0,12$ \\
\hline
\end{tabular}

Fett: Wert außerhalb des 2SD-Bereiches. *Keine STEAM-Diffusionsdaten o. MT-Daten vorhanden **Daten wurde mit STEAM_P1 Protokoll aufgenommen. Nummerierung: Patientennummer. GCC: Genu. SCC: Splenium. CC: Corpus Callosum. WMLF: Weiße Hirnsubstanz links frontal. WMLPO: Weiße Hirnsubstanz links parieto-okzipital. FA (p. u.). AD, RD, MD (Einheit in $10-3 \mathrm{~mm}^{2} \cdot \mathrm{s}^{-1}$ ), MTsat (p. u.). Alter: (in J.). Angabe der Werte: Wert \pm SD. Die aufgeführten Diffusionsdaten wurden, wenn nicht anders angegeben, mit einer STEAM-Sequenz (Protokoll STEAM_P2) aufgenommen.

Tabelle A-7: cFD-Patientin 10. Quantitative DTI- und MTsat-Daten

\begin{tabular}{|c|c|c|c|c|c|c|c|}
\hline Messung & Alter & ROI & FA & $\mathrm{AD}$ & RD & MD & MTsat \\
\hline \multirow[t]{5}{*}{1} & 0,2 & GCC & $0,69 \pm 0,03$ & $1,82 \pm 0,13$ & $0,49 \pm 0,08$ & $0,94 \pm 0,10$ & $0,82 \pm 0,07$ \\
\hline & & SCC & $0,71 \pm 0,00$ & $1,73 \pm 0,03$ & $0,44 \pm 0,01$ & $0,87 \pm 0,01$ & $0,78 \pm 0,08$ \\
\hline & & $\mathrm{CC}$ & $0,56 \pm 0,00$ & $1,64 \pm 0,03$ & $0,61 \pm 0,01$ & $0,95 \pm 0,01$ & \\
\hline & & WMLF & & & & & $0,72 \pm 0,07$ \\
\hline & & WMLPO & & & & & $0,66 \pm 0,07$ \\
\hline \multirow[t]{5}{*}{2} & 1,1 & GCC & $0,71 \pm 0,01$ & $1,44 \pm 0,10$ & $0,36 \pm 0,02$ & $0,72 \pm 0,05$ & $2,14 \pm 0,30$ \\
\hline & & SCC & $0,81 \pm 0,00$ & $1,44 \pm 0,05$ & $0,24 \pm 0,01$ & $0,64 \pm 0,02$ & $2,24 \pm 0,36$ \\
\hline & & $\mathrm{CC}$ & $0,75 \pm 0,00$ & $1,42 \pm 0,05$ & $0,32 \pm 0,01$ & $0,68 \pm 0,02$ & \\
\hline & & WMLF & & & & & $1,71 \pm 0,14$ \\
\hline & & WMLPO & & & & & $1,71 \pm 0,15$ \\
\hline \multirow[t]{5}{*}{3} & 2,3 & GCC & $0,80 \pm 0,01$ & $1,36 \pm 0,07$ & $0,25 \pm 0,02$ & $0,62 \pm 0,04$ & $2,59 \pm 0,22$ \\
\hline & & $\mathrm{SCC}$ & $0,83 \pm 0,02$ & $1,37 \pm 0,01$ & $0,21 \pm 0,03$ & $0,60 \pm 0,02$ & $2,66 \pm 0,37$ \\
\hline & & $\mathrm{CC}$ & $0,77 \pm 0,02$ & $1,33 \pm 0,01$ & $0,27 \pm 0,03$ & $0,62 \pm 0,02$ & \\
\hline & & WMLF & & & & & $2,33 \pm 0,14$ \\
\hline & & WMLPO & & & & & $2,09 \pm 0,18$ \\
\hline
\end{tabular}




\begin{tabular}{|c|c|c|c|c|c|c|c|}
\hline Messung & Alter & ROI & FA & $\mathrm{AD}$ & RD & MD & MTsat \\
\hline \multirow[t]{5}{*}{4} & 2,9 & GCC & $0,79 \pm 0,00$ & $1,38 \pm 0,01$ & $0,25 \pm 0,01$ & $0,63 \pm 0,01$ & $2,83 \pm 0,19$ \\
\hline & & SCC & $0,83 \pm 0,02$ & $1,38 \pm 0,04$ & $0,22 \pm 0,01$ & $0,61 \pm 0,01$ & $2,67 \pm 0,31$ \\
\hline & & $\mathrm{CC}$ & $0,78 \pm 0,02$ & $1,35 \pm 0,04$ & $0,26 \pm 0,01$ & $0,62 \pm 0,01$ & \\
\hline & & WMLF & & & & & $2,4 \pm 0,15$ \\
\hline & & WMLPO & & & & & $2,39 \pm 0,23$ \\
\hline \multirow[t]{5}{*}{5} & 3,4 & GCC & $0,79 \pm 0,05$ & $1,50 \pm 0,05$ & $0,29 \pm 0,07$ & $0,69 \pm 0,07$ & $2,9 \pm 0,11$ \\
\hline & & SCC & $0,77 \pm 0,01$ & $1,39 \pm 0,02$ & $0,28 \pm 0,01$ & $0,65 \pm 0,01$ & $2,85 \pm 0,17$ \\
\hline & & $\mathrm{CC}$ & $0,75 \pm 0,01$ & $1,40 \pm 0,02$ & $0,31 \pm 0,01$ & $0,67 \pm 0,01$ & \\
\hline & & WMLF & & & & & $2,56 \pm 0,14$ \\
\hline & & WMLPO & & & & & $2,36 \pm 0,23$ \\
\hline \multirow[t]{5}{*}{6} & 4,3 & GCC & $0,81 \pm 0,04$ & $1,47 \pm 0,06$ & $0,26 \pm 0,06$ & $0,66 \pm 0,05$ & $3,09 \pm 0,26$ \\
\hline & & SCC & $0,81 \pm 0,03$ & $1,39 \pm 0,09$ & $0,23 \pm 0,03$ & $0,62 \pm 0,03$ & $2,90 \pm 0,20$ \\
\hline & & $\mathrm{CC}$ & $0,78 \pm 0,03$ & $1,38 \pm 0,09$ & $0,27 \pm 0,03$ & $0,64 \pm 0,03$ & \\
\hline & & WMLF & & & & & $2,65 \pm 0,14$ \\
\hline & & WMLPO & & & & & $2,45 \pm 0,19$ \\
\hline \multirow[t]{5}{*}{7} & 5,3 & GCC & $0,82 \pm 0,02$ & $1,47 \pm 0,05$ & $0,24 \pm 0,02$ & $0,65 \pm 0,01$ & $2,91 \pm 0,17$ \\
\hline & & SCC & $0,80 \pm 0,04$ & $1,37 \pm 0,05$ & $0,25 \pm 0,05$ & $0,62 \pm 0,05$ & $2,78 \pm 0,16$ \\
\hline & & $\mathrm{CC}$ & $0,74 \pm 0,04$ & $1,35 \pm 0,05$ & $0,31 \pm 0,05$ & $0,65 \pm 0,05$ & \\
\hline & & WMLF & & & & & $2,64 \pm 0,16$ \\
\hline & & WMLPO & & & & & $2,58 \pm 0,21$ \\
\hline \multirow[t]{5}{*}{8} & 5,9 & GCC & $0,82 \pm 0,02$ & $1,33 \pm 0,04$ & $0,22 \pm 0,01$ & $0,59 \pm 0,01$ & $2,87 \pm 0,17$ \\
\hline & & SCC & $0,80 \pm 0,01$ & $1,27 \pm 0,04$ & $0,23 \pm 0,02$ & $0,58 \pm 0,03$ & $2,78 \pm 0,23$ \\
\hline & & $\mathrm{CC}$ & $0,76 \pm 0,01$ & $1,30 \pm 0,04$ & $0,27 \pm 0,02$ & $0,61 \pm 0,03$ & \\
\hline & & WMLF & & & & & $2,66 \pm 0,14$ \\
\hline & & WMLPO & & & & & $2,60 \pm 0,23$ \\
\hline \multirow[t]{5}{*}{9} & 7,0 & GCC & $0,82 \pm 0,05$ & $1,46 \pm 0,07$ & $0,24 \pm 0,06$ & $0,64 \pm 0,06$ & \\
\hline & & SCC & $0,77 \pm 0,00$ & $1,30 \pm 0,03$ & $0,27 \pm 0,01$ & $0,61 \pm 0,02$ & \\
\hline & & $\mathrm{CC}$ & $0,74 \pm 0,00$ & $1,33 \pm 0,03$ & $0,31 \pm 0,01$ & $0,65 \pm 0,02$ & \\
\hline & & WMLF & & & & & \\
\hline & & WMLPO & & & & & \\
\hline \multicolumn{8}{|l|}{ Kontrolle } \\
\hline \multirow[t]{3}{*}{ STEAM_P1 } & $1,6-$ & GCC & 0,66 & 1,54 & 0,44 & 0,80 & \\
\hline & $4,9 \mathrm{~J}$. & SCC & 0,67 & 1,49 & 0,43 & 0,78 & \\
\hline & & $\mathrm{CC}$ & 0,62 & 1,55 & 0,51 & 0,85 & \\
\hline \multirow[t]{3}{*}{ STEAM_P1 } & $5,0-$ & GCC & $0,64 \pm 0,05$ & $1,47 \pm 0,03$ & $0,46 \pm 0,07$ & $0,79 \pm 0,04$ & \\
\hline & $15,0 \mathrm{~J}$. & SCC & $0,70 \pm 0,01$ & $1,54 \pm 0,06$ & $0,40 \pm 0,01$ & $0,78 \pm 0,03$ & \\
\hline & & $\mathrm{CC}$ & $0,62 \pm 0,04$ & $1,54 \pm 0,06$ & $0,51 \pm 0,05$ & $0,85 \pm 0,05$ & \\
\hline \multirow[t]{3}{*}{ STEAM_P2 } & $1,6-$ & GCC & $0,76 \pm 0,00$ & $1,47 \pm 0,10$ & $0,31 \pm 0,04$ & $0,69 \pm 0,06$ & \\
\hline & $4,9 \mathrm{~J}$. & SCC & $0,76 \pm 0,11$ & $1,37 \pm 0,06$ & $0,31 \pm 0,13$ & $0,66 \pm 0,07$ & \\
\hline & & $\mathrm{CC}$ & $0,76 \pm 0,05$ & $1,38 \pm 0,05$ & $0,31 \pm 0,04$ & $0,67 \pm 0,04$ & \\
\hline \multirow[t]{3}{*}{ STEAM_P2 } & $5,0-$ & GCC & $0,78 \pm 0,05$ & $1,35 \pm 0,09$ & $0,28 \pm 0,04$ & $0,63 \pm 0,04$ & \\
\hline & $15,0 \mathrm{~J}$. & SCC & $0,78 \pm 0,04$ & $1,37 \pm 0,04$ & $0,28 \pm 0,04$ & $0,64 \pm 0,03$ & \\
\hline & & $\mathrm{CC}$ & $0,74 \pm 0,04$ & $1,31 \pm 0,08$ & $0,30 \pm 0,04$ & $0,64 \pm 0,04$ & \\
\hline
\end{tabular}




\begin{tabular}{|c|c|c|c|c|c|c|c|}
\hline Messung & Alter & ROI & FA & $\mathrm{AD}$ & RD & MD & MTsat \\
\hline \multirow[t]{4}{*}{ MT } & $1,6-$ & GCC & & & & & $2,71 \pm 0,38$ \\
\hline & $4,9 \mathrm{~J}$. & SCC & & & & & $2,74 \pm 0,27$ \\
\hline & & WMLF & & & & & $2,62 \pm 0,33$ \\
\hline & & WMLPO & & & & & $2,52 \pm 0,24$ \\
\hline \multirow[t]{4}{*}{ MT } & $5,0-$ & GCC & & & & & $3,05 \pm 0,19$ \\
\hline & $15,0 \mathrm{~J}$. & SCC & & & & & $2,89 \pm 0,18$ \\
\hline & & WMLF & & & & & $2,82 \pm 0,16$ \\
\hline & & WMLPO & & & & & $2,74 \pm 0,12$ \\
\hline
\end{tabular}

Fett: Wert außerhalb des 2SD-Bereiches. *Keine STEAM-Diffusionsdaten o. MT-Daten vorhanden **Daten wurde mit STEAM_P1 Protokoll aufgenommen. Nummerierung: Patientennummer. GCC: Genu. SCC: Splenium. CC: Corpus Callosum. WMLF: Weiße Hirnsubstanz links frontal. WMLPO: Weiße Hirnsubstanz links parieto-okzipital. FA (p. u.). AD, RD, MD (Einheit in $10-3 \mathrm{~mm}^{2} \cdot \mathrm{s}^{-1}$ ), MTsat (p. u.). Alter: (in J.). Angabe der Werte: Wert \pm SD. Die aufgeführten Diffusionsdaten wurden, wenn nicht anders angegeben, mit einer STEAM-Sequenz (Protokoll STEAM_P2) aufgenommen.

Tabelle A-8: cFD-Patientin 11. Quantitative DTI- und MTsat-Daten

\begin{tabular}{|c|c|c|c|c|c|c|c|}
\hline Messung & Alter & ROI & FA & $\mathrm{AD}$ & RD & MD & MTsat \\
\hline \multirow[t]{5}{*}{1} & 5,0 & GCC & $0,93 \pm 0,00$ & $1,84 \pm 0,10$ & $0,12 \pm 0,01$ & $0,69 \pm 0,04$ & $2,05 \pm 0,48$ \\
\hline & & SCC & $0,91 \pm 0,02$ & $1,97 \pm 0,04$ & $0,16 \pm 0,04$ & $0,77 \pm 0,04$ & $2,17 \pm 0,32$ \\
\hline & & $\mathrm{CC}$ & $0,82 \pm 0,02$ & $1,87 \pm 0,04$ & $0,30 \pm 0,04$ & $0,82 \pm 0,04$ & \\
\hline & & WMLF & & & & & $1,93 \pm 0,14$ \\
\hline & & WMLPO & & & & & $1,97 \pm 0,22$ \\
\hline \multirow[t]{5}{*}{2} & 5,5 & GCC & $0,88 \pm 0,03$ & $1,95 \pm 0,03$ & $0,21 \pm 0,05$ & $0,79 \pm 0,04$ & $2,38 \pm 0,61$ \\
\hline & & SCC & $0,83 \pm 0,05$ & $2,05 \pm 0,12$ & $0,33 \pm 0,11$ & $0,90 \pm 0,11$ & $2,23 \pm 0,31$ \\
\hline & & $\mathrm{CC}$ & $0,79 \pm 0,05$ & $1,89 \pm 0,12$ & $0,35 \pm 0,11$ & $0,86 \pm 0,11$ & \\
\hline & & WMLF & & & & & $2,12 \pm 0,14$ \\
\hline & & WMLPO & & & & & $1,88 \pm 0,16$ \\
\hline \multirow[t]{5}{*}{3} & 6,0 & GCC & $0,86 \pm 0,02$ & $1,82 \pm 0,08$ & $0,22 \pm 0,03$ & $0,75 \pm 0,04$ & $2,59 \pm 0,21$ \\
\hline & & SCC & $0,85 \pm 0,06$ & $1,95 \pm 0,04$ & $0,27 \pm 0,12$ & $0,83 \pm 0,09$ & $2,46 \pm 0,17$ \\
\hline & & $\mathrm{CC}$ & $0,79 \pm 0,06$ & $1,94 \pm 0,04$ & $0,39 \pm 0,12$ & $0,91 \pm 0,09$ & \\
\hline & & WMLF & & & & & $2,26 \pm 0,13$ \\
\hline & & WMLPO & & & & & $2,07 \pm 0,08$ \\
\hline \multirow[t]{5}{*}{4} & 6,5 & GCC & $0,81 \pm 0,03$ & $1,86 \pm 0,10$ & $0,31 \pm 0,07$ & $0,83 \pm 0,08$ & $2,14 \pm 0,19$ \\
\hline & & SCC & $0,80 \pm 0,04$ & $1,95 \pm 0,15$ & $0,36 \pm 0,05$ & $0,89 \pm 0,06$ & $2,17 \pm 0,16$ \\
\hline & & $\mathrm{CC}$ & $0,79 \pm 0,04$ & $1,90 \pm 0,15$ & $0,36 \pm 0,05$ & $0,87 \pm 0,06$ & \\
\hline & & WMLF & & & & & $2,04 \pm 0,11$ \\
\hline & & WMLPO & & & & & $2,07 \pm 0,09$ \\
\hline \multirow[t]{5}{*}{5} & 7,0 & GCC & $0,81 \pm 0,02$ & $1,95 \pm 0,02$ & $0,33 \pm 0,02$ & $0,87 \pm 0,00$ & $2,21 \pm 0,20$ \\
\hline & & SCC & $0,80 \pm 0,00$ & $2,04 \pm 0,07$ & $0,39 \pm 0,01$ & $0,94 \pm 0,03$ & $2,14 \pm 0,20$ \\
\hline & & $\mathrm{CC}$ & $0,72 \pm 0,00$ & $1,83 \pm 0,07$ & $0,45 \pm 0,01$ & $0,91 \pm 0,03$ & \\
\hline & & WMLF & & & & & $2,05 \pm 0,12$ \\
\hline & & WMLPO & & & & & $2,29 \pm 0,10$ \\
\hline
\end{tabular}




\begin{tabular}{|c|c|c|c|c|c|c|c|}
\hline Messung & Alter & ROI & FA & $\mathrm{AD}$ & RD & MD & MTsat \\
\hline \multicolumn{8}{|l|}{ Kontrolle } \\
\hline \multirow[t]{3}{*}{ EPI } & $1,6-$ & GCC & 0,80 & 1,86 & 0,32 & 0,84 & \\
\hline & $4,9 \mathrm{~J}$. & SCC & 0,77 & 2,08 & 0,45 & 0,99 & \\
\hline & & $\mathrm{CC}$ & 0,74 & 1,86 & 0,42 & 0,90 & \\
\hline \multirow[t]{3}{*}{ EPI } & $5,0-$ & GCC & $0,85 \pm 0,02$ & $1,71 \pm 0,14$ & $0,23 \pm 0,02$ & $0,73 \pm 0,03$ & \\
\hline & $15,0 \mathrm{~J}$. & SCC & $0,87 \pm 0,02$ & $1,84 \pm 0,24$ & $0,23 \pm 0,01$ & $0,77 \pm 0,08$ & \\
\hline & & $\mathrm{CC}$ & $0,83 \pm 0,02$ & $1,70 \pm 0,21$ & $0,25 \pm 0,01$ & $0,73 \pm 0,07$ & \\
\hline \multirow[t]{4}{*}{ MT } & $1,6-$ & GCC & & & & & $2,71 \pm 0,38$ \\
\hline & $4,9 \mathrm{~J}$. & SCC & & & & & $2,74 \pm 0,27$ \\
\hline & & WMLF & & & & & $2,62 \pm 0,33$ \\
\hline & & WMLPO & & & & & $2,52 \pm 0,24$ \\
\hline \multirow[t]{4}{*}{ MT } & $5,0-$ & GCC & & & & & $3,05 \pm 0,19$ \\
\hline & $15,0 \mathrm{~J}$. & SCC & & & & & $2,89 \pm 0,18$ \\
\hline & & WMLF & & & & & $2,82 \pm 0,16$ \\
\hline & & WMLPO & & & & & $2,74 \pm 0,12$ \\
\hline
\end{tabular}

Fett: Wert außerhalb des 2SD-Bereiches. *Keine EPI-Diffusionsdaten o. MT-Daten vorhanden. Nummerierung: Patientennummer. GCC: Genu. SCC: Splenium. CC: Corpus Callosum. WMLF: Weiße Hirnsubstanz links frontal. WMLPO: Weiße Hirnsubstanz links parieto-okzipital. FA (p. u.). AD, RD, MD (Einheit in 10-3 $\mathrm{mm}^{2} \cdot \mathrm{s}^{-1}$ ), MTsat (p. u.). Alter: (in J.). Angabe der Werte: Wert \pm SD. Die aufgeführten Diffusionsdaten wurden, wenn nicht anders angegeben, mit einer EPISequenz aufgenommen.

Tabelle A-9: cFD-Patient 12. Quantitative DTI- und MTsat-Daten

\begin{tabular}{|c|c|c|c|c|c|c|c|}
\hline Messung & Alter & ROI & FA & $\mathrm{AD}$ & RD & MD & MTsat \\
\hline \multirow[t]{5}{*}{$1 *$} & 1,3 & GCC & & & & & $1,72 \pm 0,47$ \\
\hline & & SCC & & & & & $1,29 \pm 0,76$ \\
\hline & & CC & & & & & \\
\hline & & WMLF & & & & & $1,21 \pm 0,07$ \\
\hline & & WMLPO & & & & & $1,05 \pm 0,13$ \\
\hline \multirow[t]{5}{*}{2} & 1,8 & GCC & $0,76 \pm 0,03$ & $1,76 \pm 0,14$ & $0,39 \pm 0,08$ & $0,85 \pm 0,09$ & $1,62 \pm 0,61$ \\
\hline & & SCC & $0,79 \pm 0,05$ & $2,20 \pm 0,10$ & $0,42 \pm 0,10$ & $1,01 \pm 0,08$ & $1,75 \pm 0,64$ \\
\hline & & $\mathrm{CC}$ & $0,75 \pm 0,05$ & $1,86 \pm 0,10$ & $0,36 \pm 0,10$ & $0,86 \pm 0,08$ & \\
\hline & & WMLF & & & & & $1,56 \pm 0,15$ \\
\hline & & WMLPO & & & & & $1,51 \pm 0,16$ \\
\hline \multirow[t]{5}{*}{3} & 2,2 & GCC & $0,80 \pm 0,02$ & $1,78 \pm 0,07$ & $0,31 \pm 0,04$ & $0,80 \pm 0,05$ & $2,22 \pm 0,49$ \\
\hline & & SCC & $0,81 \pm 0,03$ & $2,16 \pm 0,10$ & $0,38 \pm 0,08$ & $0,97 \pm 0,09$ & $1,94 \pm 0,80$ \\
\hline & & $\mathrm{CC}$ & $0,77 \pm 0,03$ & $1,84 \pm 0,10$ & $0,38 \pm 0,08$ & $0,87 \pm 0,09$ & \\
\hline & & WMLF & & & & & $1,78 \pm 0,13$ \\
\hline & & WMLPO & & & & & $1,72 \pm 0,17$ \\
\hline \multirow[t]{5}{*}{4} & 2,9 & GCC & $0,82 \pm 0,02$ & $1,71 \pm 0,12$ & $0,21 \pm 0,04$ & $0,57 \pm 0,05$ & $2,63 \pm 0,29$ \\
\hline & & SCC & $0,85 \pm 0,02$ & $2,13 \pm 0,10$ & $0,22 \pm 0,02$ & $0,68 \pm 0,02$ & $2,16 \pm 0,62$ \\
\hline & & CC & $0,78 \pm 0,02$ & $1,91 \pm 0,10$ & $0,37 \pm 0,02$ & $0,88 \pm 0,02$ & \\
\hline & & WMLF & & & & & $1,95 \pm 0,23$ \\
\hline & & WMLPO & & & & & $1,84 \pm 0,18$ \\
\hline
\end{tabular}




\begin{tabular}{|c|c|c|c|c|c|c|c|}
\hline Messung & Alter & ROI & FA & $\mathrm{AD}$ & RD & MD & MTsat \\
\hline \multirow[t]{5}{*}{5} & 3,4 & GCC & $0,82 \pm 0,02$ & $1,75 \pm 0,03$ & $0,28 \pm 0,02$ & $0,77 \pm 0,01$ & $2,77 \pm 0,29$ \\
\hline & & SCC & $0,78 \pm 0,01$ & $2,05 \pm 0,22$ & $0,40 \pm 0,06$ & $0,95 \pm 0,11$ & $2,59 \pm 0,46$ \\
\hline & & $\mathrm{CC}$ & $0,74 \pm 0,01$ & $1,82 \pm 0,22$ & $0,41 \pm 0,06$ & $0,88 \pm 0,11$ & \\
\hline & & WMLF & & & & & $2,30 \pm 0,16$ \\
\hline & & WMLPO & & & & & $2,12 \pm 0,14$ \\
\hline \multirow[t]{5}{*}{$6^{*}$} & 3,9 & GCC & & & & & $2,89 \pm 0,26$ \\
\hline & & SCC & & & & & $2,57 \pm 0,65$ \\
\hline & & $\mathrm{CC}$ & & & & & \\
\hline & & WMLF & & & & & $2,38 \pm 0,18$ \\
\hline & & WMLPO & & & & & $2,09 \pm 0,19$ \\
\hline \multirow[t]{5}{*}{7} & 4,5 & GCC & $0,85 \pm 0,04$ & $1,75 \pm 0,05$ & $0,23 \pm 0,06$ & $0,74 \pm 0,05$ & $2,79 \pm 0,42$ \\
\hline & & SCC & $0,81 \pm 0,08$ & $2,03 \pm 0,07$ & $0,33 \pm 0,12$ & $0,90 \pm 0,06$ & $2,79 \pm 0,44$ \\
\hline & & $\mathrm{CC}$ & $0,76 \pm 0,08$ & $1,82 \pm 0,07$ & $0,37 \pm 0,12$ & $0,85 \pm 0,06$ & \\
\hline & & WMLF & & & & & $2,54 \pm 0,16$ \\
\hline & & WMLPO & & & & & $2,35 \pm 0,15$ \\
\hline \multirow[t]{5}{*}{$8^{*}$} & 5,0 & GCC & $0,76 \pm 0,02$ & $1,78 \pm 0,09$ & $0,37 \pm 0,06$ & $0,84 \pm 0,07$ & $3,00 \pm 0,18$ \\
\hline & & SCC & $0,67 \pm 0,02$ & $2,11 \pm 0,09$ & $0,62 \pm 0,03$ & $1,12 \pm 0,04$ & $2,99 \pm 0,26$ \\
\hline & & $\mathrm{CC}$ & $0,70 \pm 0,02$ & $1,83 \pm 0,09$ & $0,49 \pm 0,03$ & $0,93 \pm 0,04$ & \\
\hline & & WMLF & & & & & $2,64 \pm 0,10$ \\
\hline & & WMLPO & & & & & $2,52 \pm 0,12$ \\
\hline \multirow[t]{5}{*}{9} & 5,5 & GCC & $0,83 \pm 0,09$ & $1,74 \pm 0,10$ & $0,27 \pm 0,15$ & $0,76 \pm 0,13$ & $3,14 \pm 0,20$ \\
\hline & & SCC & $0,70 \pm 0,05$ & $2,06 \pm 0,06$ & $0,56 \pm 0,08$ & $1,06 \pm 0,05$ & $3,04 \pm 0,22$ \\
\hline & & $\mathrm{CC}$ & $0,74 \pm 0,05$ & $1,85 \pm 0,06$ & $0,43 \pm 0,08$ & $0,90 \pm 0,05$ & \\
\hline & & WMLF & & & & & $2,65 \pm 0,11$ \\
\hline & & WMLPO & & & & & $2,35 \pm 0,07$ \\
\hline \multirow[t]{5}{*}{$10^{*}$} & 6,2 & GCC & $0,79 \pm 0,02$ & $1,76 \pm 0,10$ & $0,33 \pm 0,04$ & $0,81 \pm 0,05$ & $3,13 \pm 0,20$ \\
\hline & & SCC & $0,69 \pm 0,06$ & $2,02 \pm 0,05$ & $0,56 \pm 0,11$ & $1,04 \pm 0,08$ & $3,02 \pm 0,22$ \\
\hline & & $\mathrm{CC}$ & $0,71 \pm 0,06$ & $1,75 \pm 0,05$ & $0,44 \pm 0,11$ & $0,88 \pm 0,08$ & \\
\hline & & WMLF & & & & & $2,80 \pm 0,13$ \\
\hline & & WMLPO & & & & & $2,67 \pm 0,14$ \\
\hline \multicolumn{8}{|l|}{ Kontrolle } \\
\hline \multirow[t]{3}{*}{ EPI } & $1,6-$ & GCC & 0,80 & 1,86 & 0,32 & 0,84 & \\
\hline & $4,9 \mathrm{~J}$. & SCC & 0,77 & 2,08 & 0,45 & 0,99 & \\
\hline & & CC & 0,74 & 1,86 & 0,42 & 0,90 & \\
\hline \multirow[t]{3}{*}{ EPI } & $5,0-$ & GCC & $0,85 \pm 0,02$ & $1,71 \pm 0,14$ & $0,23 \pm 0,02$ & $0,73 \pm 0,03$ & \\
\hline & $15,0 \mathrm{~J}$. & SCC & $0,87 \pm 0,02$ & $1,84 \pm 0,24$ & $0,23 \pm 0,01$ & $0,77 \pm 0,08$ & \\
\hline & & $\mathrm{CC}$ & $0,83 \pm 0,02$ & $1,70 \pm 0,21$ & $0,25 \pm 0,01$ & $0,73 \pm 0,07$ & \\
\hline \multirow[t]{4}{*}{ MT } & $1,6-$ & GCC & & & & & $2,71 \pm 0,38$ \\
\hline & 4,9 J. & SCC & & & & & $2,74 \pm 0,27$ \\
\hline & & WMLF & & & & & $2,62 \pm 0,33$ \\
\hline & & WMLPO & & & & & $2,52 \pm 0,24$ \\
\hline \multirow[t]{4}{*}{ MT } & $5,0-$ & GCC & & & & & $3,05 \pm 0,19$ \\
\hline & $15,0 \mathrm{~J}$. & SCC & & & & & $2,89 \pm 0,18$ \\
\hline & & WMLF & & & & & $2,82 \pm 0,16$ \\
\hline & & WMLPO & & & & & $2,74 \pm 0,12$ \\
\hline
\end{tabular}


Fett: Wert außerhalb des 2SD-Bereiches. *Keine EPI-Diffusionsdaten o. MT-Daten vorhanden Nummerierung: Patientennummer. GCC: Genu. SCC: Splenium. CC: Corpus Callosum. WMLF: Weiße Hirnsubstanz links frontal. WMLPO: Weiße Hirnsubstanz links parieto-okzipital. FA (p. u.). AD, RD, MD (Einheit in 10-3 $\mathrm{mm}^{2} \cdot \mathrm{s}^{-1}$ ), MTsat (p. u.). Alter: (in J.). Angabe der Werte: Wert \pm SD. Die aufgeführten Diffusionsdaten wurden, wenn nicht anders angegeben, mit einer EPISequenz aufgenommen. 


\section{Danksagung}

Mein Dank gilt PD Dr. Steffi Dreha-Kulaczewski, Dr. Sabine Hofer und PD Dr. Peter Dechent für die Bereitstellung des Themas und die Möglichkeit diese Dissertation in der Klinik für Kinder- und Jugendmedizin in Kooperation mit dem Institut für Kognitive Neurologie sowie dem Göttinger Max-Planck-Institut für biophysikalische Chemie, durchführen zu können. Außerdem danke ich ihnen für die großartige Unterstützung, Betreuung und Motivation bei der Realisierung der Arbeit sowie die vielfache Durchsicht und konstruktive Kritik beim Voranbringen der Dissertationssschrift.

Ohne die Unterstützung der Mitarbeiterinnen und Mitarbeiter der Forschergruppe „MR Forschung in der Neurologie und Psychiatrie“ sowie des Max-Planck-Instituts für biophysikalische Chemie wäre diese Arbeit ebenfalls nicht möglich gewesen. Hier möchte ich mich insbesondere bei Prof. Dr. Melanie Wilke, Severin Heumüller, Dr. Iris Steinmann, Ilona Pfahlert und Regina Vinnen aus der MR Forschung sowie Prof. Dr. Jens Frahm, Dr. Dirk Voit, Dr. Andreas Merrem, Dr. Volkert Brar Roeloffs, Jost Michael Kollmeier, Kurt Böhm, Lutz Präkelt und Sylke Wallbrecht vom Max-Planck-Institut bedanken. Ebenfalls möchte ich mich für die fachliche und persönliche Unterstützung der Arbeit bei meiner Familie und meinen Freunden bedanken.

Darüber hinaus danke ich Prof. Dr. Birgit Ertl-Wagner und PD Dr. Mark Born für die freundliche Zurverfügungstellung der MRT-Aufnahmen im ersten Kapitel. 